\title{
OBSERVATIONS OF THE DYNAMICS AND ACOUSTICS OF TRAVELLING BUBBLE CAVITATION
}

\author{
Thesis by \\ Steven Louis Ceccio \\ Division of Engineering and Applied Science
}

\author{
In Partial Fulfillment \\ of the Requirements for the Degree of \\ Doctor of Philosophy
}
California Institute of Technology
Pasadena, California
1990

(Submitted May 25, 1990) 
(C) 1990

Steven Louis Ceccio

All Rights Reserved 


\section{ACKNOWLEDGEMENTS}

I would like to express my deepest gratitude to my advisor, Professor Christopher Brennen. His professional and personal guidance made the completion of this work possible. Furthermore, I would like to thank Professor Allan Acosta, whose advice and considerations proved invaluable. The continued interest Professors Rolf Sabersky and Thomas Caughey have expressed in this work is greatly appreciated.

This thesis relied heavily upon the professional staff of Caltech, and I thank all those people that contributed. I would especially like to thank John Lee for his assistance in the electrical design, Marty Gould for his expert machining and patient advice, and Cecilia Lin for her preparation of the figures. I would also like to express my gratitude to Jackie Beard and Dana Young for their support and encouragement.

I gratefully acknowledge the help of my fellow graduate students, especially Ron Franz, Douglas Hart, Sanjay Kumar, and Adiel Guinzburg. I would also like to acknowledge the numerous undergraduates who have assisted in this work with special thanks to Imran Kizilbash and Dong-Su Kim.

Funding for this work was provided by the Office of Naval Research under contract number N-00014-85-K-0397, and the Francis J. Cole Foundation and the ARCS Foundation also provivided financial assistance. This support was essential to the completion of this work.

Last, but certainly not least, I thank my family for their unfailing encouragement and understanding. This work would not have been possible without the loving support of my parents, and I therefore dedicate it to them. 


\begin{abstract}
Individual travelling cavitation bubbles generated on two axisymmetric headforms were detected using a surface electrode probe. The growth and collapse of the bubbles, almost all of which were quasi-spherical caps moving close to the headform surface, were studied photographically. Although the growth patterns for the two headforms were similar, the collapse mechanisms were quite different. These differences were related to the pressure fields and viscous flow patterns associated with each headform. Measurements of the acoustic impulse generated by the bubble collapse were analyzed and found to correlate with the maximum volume of the bubble for each headform. Numerical solutions of the Rayleigh-Plesset equation were generated for the same flows and compared with the experimental data. The experiments revealed that for smaller bubbles the impulse-volume relationship is determinate, but for larger bubbles the impulse becomes more uncertain. The theoretical impulse was at least a factor of two greater than the measured impulse, and the impulse-volume relationship was related to the details of the collapse mechanism. Acoustic emission of individual cavitation events was spectrally analyzed and the results were compared with relevant theoretical and emperical predictions. Finally, the cavitation nuclei flux was measured and compared to the cavitation event rate and the bubble maximum size distribution through the use of a simple model. The nuclei number distribution was found to vary substantially with tunnel operating conditions, and changes in the nuclei number distribution significantly influenced the cavitation event rate and bubble maximum size distribution. The model estimated the cavitation event rate but failed to predict the bubble maximum size distribution. With the above theoretical and experimental results, the cavitation rate and resulting noise production may be estimated from a knowledge of the non-cavitating flow and the free stream nuclei number distribution.
\end{abstract}




\section{TABLE OF CONTENTS}

Title Page $\quad$ i

Copyright $\quad$ ii

Acknowledgements $\quad$ iii

Abstract $\quad$ iv

Table of Contents $\quad$ v

Nomenclature $\quad$ ix

List of Figures $\quad$ xii

1. Introduction 1

1.1 Current Knowledge of Bubble Cavitation 1

1.2 Current Knowledge of Cavitation Noise 2

1.3 Outline of Research 3

2. Experimental Equipment 5

2.1 Water Tunnel 5

2.2 Test Bodies 5

2.3 Hydrophone System 6

2.3.1 Hydrophone Setup 6

2.3.2 Hydrophone Calibration 6

$\begin{array}{ll}2.4 \text { Nuclei Counter } & 7\end{array}$

3. Surface Electrode Probe 14

3.1 Introduction 14

$\begin{array}{ll}3.2 \text { Principle of Operation } & 14\end{array}$ 
3.3 Electrode Geometry 15

$\begin{array}{ll}\text { 3.3.1 Patch Geometry } & 15\end{array}$

$\begin{array}{ll}\text { 3.3.2 Circular Geometry } & 16\end{array}$

$\begin{array}{ll}3.4 \text { Calibration } & 16\end{array}$

$\begin{array}{ll}\text { 3.5 Post Signal Processing } & 17\end{array}$

$\begin{array}{ll}3.6 \text { Conclusion } & 18\end{array}$

4. Observations of Single Cavitation Bubbles 34

4.1 Introduction 34

4.2 General Observations 34

4.3 Schiebe Body Results 36

4.4 I.T.T.C. Body Results 36

$\begin{array}{ll}4.5 \text { Conclusions } & 38\end{array}$

5. Theoretical Analysis of the Dynamics of Single Cavitation Bubbles 54

$\begin{array}{ll}5.1 \text { Introduction } & 54\end{array}$

5.2 Bubble Dynamics $\quad 55$

5.2.1 Bubble Stability $\quad 55$

5.2.2 Bubble Growth 57

$\begin{array}{ll}\text { 5.2.3 Bubble Collapse } & 57\end{array}$

5.3 Acoustic Emission of Cavitating Bubbles 60

$\begin{array}{ll}\text { 5.3.1 General Considerations } & 60\end{array}$

5.3.2 Emission During Bubble Collapse $\quad 60$

5.4 Spectral Analysis of Bubble Acoustic Emission 61 
$\begin{array}{ll}5.5 \text { Conclusions } & 62\end{array}$

6. Measurement of the Acoustic Emission of Single Cavitation Bubbles 67

$\begin{array}{ll}6.1 \text { Introduction } & 67\end{array}$

6.2 Schiebe Body Results 68

6.3 I.T.T.C. Body Results $\quad 69$

$\begin{array}{ll}\text { 6.4 Comparison with Numerical Results } & 70\end{array}$

6.5 Spectral Analysis of Bubble Emission $\quad 72$

$\begin{array}{ll}6.6 \text { Conclusions } & 74\end{array}$

7. Observations of Cavitation Event Rate and Bubble Maximum Size Distribu$\begin{array}{ll}\text { tion } & 100\end{array}$

$\begin{array}{ll}7.1 \text { Introduction } & 100\end{array}$

$\begin{array}{ll}7.2 \text { Experimental Measurements } & 101\end{array}$

$\begin{array}{ll}\text { 7.2.1 Cavitation Event Rate and Statistics } & 101\end{array}$

$\begin{array}{ll}\text { 7.2.2 Bubble Maximum Size Distribution } & 102\end{array}$

$\begin{array}{ll}\text { 7.2.3 Nuclei Number Distribution } & 102\end{array}$

$\begin{array}{ll}7.3 \text { Theoretical Considerations } & 103\end{array}$

$\begin{array}{ll}\text { 7.3.1 Cavitation Event Rate } & 103\end{array}$

$\begin{array}{ll}\text { 7.3.2 Bubble Maximum Size Distribution } & 104\end{array}$

7.4 Comparison of Predicted and Measured Data 105

$\begin{array}{ll}7.5 \text { Conclusions } & 107\end{array}$

8. Summary and Conclusions 114

$\begin{array}{ll}8.1 \text { Summary } & 114\end{array}$

$\begin{array}{ll}8.2 \text { Conclusions } & 117\end{array}$ 
Appendix A. Technical Description of the Surface Electrode Probe 


\section{NOMENCLATURE}

$A\left(R_{O}\right) \quad$ streamtube capture area for given nuclei

$c \quad$ sonic speed of fluid

$C \quad$ sonic speed of fluid at the bubble wall

$C_{P} \quad$ pressure coefficient, $\left(P-P_{0}\right) /\left(\frac{1}{2} \rho U^{2}\right)$

$C_{P A} \quad$ average pressure coefficient in region below vapor pressure

$C_{P C} \quad$ average pressure coefficient in the region of bubble collapse

$C_{P M} \quad$ minimum pressure coefficient on body surface

$C_{P T} \quad$ minimum pressure coefficient on streamtube

$d \quad$ distance bubble travels in region below vapor pressure

$D_{B} \quad$ base diameter or bubble measured by electrode system

$E \quad$ electrode probe signal

$E_{P} \quad$ peak electrode probe signal

$f \quad$ frequency

$h \quad$ fluid enthalpy

$H \quad$ fluid enthalpy at bubble wall

I measured acoustic impulse

$I^{*} \quad$ dimensionless acoustic impulse

$L \quad$ attached cavity length

$M \quad$ Mach number

$N\left(R_{O}\right) \quad$ free stream nuclei distribution

$P(R) \quad$ pressure at bubble wall

$\operatorname{Pr}_{O}\left(R_{M}\right) \max$. bubble volume distribution associated with nuclei of size $R_{O}$

$\operatorname{Pr}\left(R_{M}\right)$ maximum bubble volume distribution

$\operatorname{Pr}(\Delta T) \quad \Delta T$ distribution

$P_{A} \quad$ acoustic pressure

$P_{C} \quad$ pressure in region of collapse

$P_{G} \quad$ non-condensable gas pressure 
$P_{G O} \quad$ non-condensable gas pressure at equilibrium nucleus radius

$P_{G M} \quad$ non-condensable gas pressure at maximum bubble radius

$P_{O} \quad$ freestream pressure

$P_{S} \quad$ pressure peak of shock wave

$P_{V} \quad$ water vapor pressure

$r \quad$ acoustic path length

$R(t) \quad$ calculated bubble radius

$R_{C} \quad$ critical nuclei radius

$R_{B} \quad$ headform radius at $C_{P M}$

$R_{H} \quad$ headform radius

$R_{L} \quad$ bubble radius along trajectory

$R_{m} \quad$ cavitation bubble minimum radius

$R_{M} \quad$ cavitation bubble maximum radius

$R_{M R} \quad$ cavitation bubble maximum reduced radius

$R_{O} \quad$ nuclei radius

Re Reynolds number based on diameter of the body, $U D / \nu$

$s \quad$ streamwise coordinate measured along the body surface from the stagnation point

$S \quad$ water surface tension

$S_{E} \quad$ electrode spectral coefficients, attached cavity

$S_{H} \quad$ acoustic pressure spectral coefficients, attached cavity

$S_{P} \quad$ acoustic pressure spectral coefficients, bubble cavitation

$t \quad$ time

$t_{1}, t_{2} \quad$ integration limits for experimental impulse

$T \quad$ acoustic pulse duration

$T^{*} \quad$ calculated dimensionless pulse duration

$u \quad$ fluid velocity

$U \quad$ free stream velocity

$V(t) \quad$ calculated bubble volume

$V_{B} \quad$ measured bubble volume based on $D_{B}$ 


$\begin{array}{ll}W e & \text { Weber number, } \rho U^{2} R_{H} / S \\ \alpha & \text { scaling factor for pulse duration relationship } \\ \beta & \text { scaling factor for critical nuclei radius relation } \\ \tau & \text { ratio of specific heats for non-condensable gas } \\ \Delta T & \text { time between cavitation events } \\ \delta \tau_{d R / d t=0} & \text { time scale for rebound of bubble in an incompressible fluid } \\ \kappa & \text { headform radius of curvature at } C_{P M} \\ \nu & \text { water viscosity } \\ \rho & \text { water density } \\ \sigma & \text { cavitation number, }\left(P-P_{V}\right) /\left(\frac{1}{2} \rho U^{2}\right) \\ \sigma_{i} & \text { travelling bubble cavitation inception index } \\ \sigma_{a c} & \text { attached cavity formation index } \\ \theta & \text { shock wave time constant } \\ \Theta & \text { cavitation event rate } \\ \tau_{C} & \text { bubble collapse time } \\ \tau_{G} & \text { bubble growth time }\end{array}$




\section{LIST OF FIGURES}

Figure 2.1 Schematic diagram of the Caltech Low Turbulence Water Tunnel.

Figure 2.2 Profile of the Schiebe and I.T.T.C. headform.

Figure 2.3 Photograph of the I.T.T.C. headform mounted in the L.T.W.T. test section on the two bladed sting.

Figure 2.4 Schematic diagram of the bubble cavitation experiment.

Figure 2.5 Receiving response of ITC-1042 hydrophone.

Figure 2.6 Dual signal trace from two ITC-1042 hydrophones. The top signal is from the internal hydrophopne, the bottom signal is from the external hydrophone.

Figure 3.1 Schematic diagram of Schiebe headform with patch electrodes.

Figure 3.2 Scheibe headform with patch electrodes.

Figure 3.3 Schematic diagram of the I.T.T.C. headform with patch electrodes.

Figure 3.4 I.T.T.C. headform with patch electrodes.

Figure 3.5 Example probe output signal for patch electrodes. Bubbles generated on the I.T.T.C. headform at $\sigma=0.50$ and $\sigma=0.42$ at $U=8.3 \mathrm{~m} / \mathrm{s}$.

Figure 3.6 Schematic diagram of Schiebe headform with circular electrodes.

Figure 3.7 Scheibe headform with circular electrodes.

Figure 3.8 Schematic diagram of the I.T.T.C. headform with circular electrodes.

Figure 3.9 I.T.T.C. headform with circular electrodes.

Figure 3.10 Illustration of base diameter measurement. As the bubble passes over the electrode, a percentage of the electrode circumference is covered (a), and this is proportional to the electrode signal (b). The maximum of the probe signal, $E_{P}$ is proportional to the maximum base diameter, $D_{B}$.

Figure 3.11 Example calibration curve for circular electrode system. Curve is for 
I.T.T.C. body.

Figure 3.12 Schematic diagram of patch electrode experiment.

Figure 3.13 Schematic diagram of bubble statistics experiment for circular electrodes.

Figure 3.14 Schematic diagram of bubble acoustics experiment for circular electrodes.

Figure 3.15 Photograph of electronic equipment.

Figure 4.1 Pressure distributions on the I.T.T.C body and the Schiebe body.

Figure 4.2 Schematic diagram of typical bubble trajectory on the Schiebe headform.

Figure 4.3 Series of photographs detailing typical bubble trajectory on the Schiebe headform, $U=9 \mathrm{~m} / \mathrm{s}$ and $\sigma=0.45$.

Figure 4.4 Schematic diagram of typical bubble trajectory on the I.T.T.C. headform.

Figure 4.5 Series of photographs detailing typical bubble trajectory on the I.T.T.C. headform, $U=8.7 \mathrm{~m} / \mathrm{s}$ and $\sigma=0.45$.

Figure 4.6 Series of photographs detailing bubbles with tails the I.T.T.C. headform, $U=9 \mathrm{~m} / \mathrm{s}$ and $\sigma=0.42$.

Figure 4.7 Photographs of bubbles taken by Knapp and Hollander.

Figure 4.8 Typical bubble trajectory observed by Knapp and Hollander.

Figure 4.9 Schiebe body isobars and typical bubble profile.

Figure 4.10 Schematic diagram of typical bubble collapse mechanism on the Schiebe headform.

Figure 4.11 Series of photographs detailing typical bubble collapse mechanism on the Schiebe headform, $U=9 \mathrm{~m} / \mathrm{s}$ and $\sigma=0.45$. 
xiv

Figure 4.12 Schematic diagram of typical bubble collapse mechanism on the I.T.T.C. headform.

Figure 4.13 Series of photographs detailing typical bubble collapse mechanism on the I.T.T.C. headform, $U=8.7 \mathrm{~m} / \mathrm{s}$ and $\sigma=0.45$.

Figure 5.1 Pressure history a nucleus would experience passing near the Schiebe or I.T.T.C. headform.

Figure 5.2 Numerical calculation of the bubble maximum radius as a function of nucleus radius for nuclei passing near the I.T.T.C. headform.

Figure 5.3 Crital nuclei radius as a function of flow parameters for nuclei passing near the I.T.T.C. headform.

Figure 6.1 Typical cavitation bubble noise emission. The bubble was generated on the Schiebe body at $U=9 \mathrm{~m} / \mathrm{s}$ and $\sigma=0.45$

Figure 6.2 Two examples of typical cavitation initial noise pulses. The bubbles were generated on the I.T.T.C. headform at $\sigma=0.45$ and $U=8.7 \mathrm{~m} / \mathrm{s}$.

Figure 6.3 Impulse v. maximum bubble volume for Schiebe body, $U=9 \mathrm{~m} / \mathrm{s}$, $\sigma=0.42$.

Figure 6.4 Impulse v. maximum bubble volume for Schiebe body, $U=9 \mathrm{~m} / \mathrm{s}$, $\sigma=0.45$.

Figure 6.5 Impulse v. maximum bubble volume for Schiebe body, $U=9 \mathrm{~m} / \mathrm{s}$, $\sigma=0.50$.

Figure 6.6 Impulse v. maximum bubble volume for Schiebe body, $U=9 \mathrm{~m} / \mathrm{s}$, $\sigma=0.55$.

Figure 6.7 Averaged impulse v. maximum bubble volume for Schiebe body, $U=9 \mathrm{~m} / \mathrm{s}, \sigma=0.42$.

Figure 6.8 Averaged impulse v. maximum bubble volume for Schiebe body, $U=9 \mathrm{~m} / \mathrm{s}, \sigma=0.45$. 
Figure 6.9 Averaged impulse v. maximum bubble volume for Schiebe body, $U=9 \mathrm{~m} / \mathrm{s}, \sigma=0.50$.

Figure 6.10 Averaged impulse v. maximum bubble volume for Schiebe body, $U=9 \mathrm{~m} / \mathrm{s}, \sigma=0.55$.

Figure 6.11 Impulse v. maximum bubble volume for I.T.T.C. body, $U=8.7 \mathrm{~m} / \mathrm{s}$, $\sigma=0.45$.

Figure 6.12 Impulse v. maximum bubble volume for I.T.T.C. body, $U=8.7 \mathrm{~m} / \mathrm{s}$, $\sigma=0.48$.

Figure 6.13 Impulse v. maximum bubble volume for I.T.T.C. body, $U=8.7 \mathrm{~m} / \mathrm{s}$, $\sigma=0.50$.

Figure 6.14 Impulse v. maximum bubble volume for I.T.T.C. body, $U=8.7 \mathrm{~m} / \mathrm{s}$, $\sigma=0.53$.

Figure 6.15 Averaged impulse v. maximum bubble volume for I.T.T.C. body, $U=8.7 \mathrm{~m} / \mathrm{s}, \sigma=0.45$.

Figure 6.16 Averaged impulse v. maximum bubble volume for I.T.T.C. body, $U=8.7 \mathrm{~m} / \mathrm{s}, \sigma=0.48$.

Figure 6.17 Averaged impulse v. maximum bubble volume for I.T.T.C. body, $U=8.7 \mathrm{~m} / \mathrm{s}, \sigma=0.50$.

Figure 6.18 Averaged impulse v. maximum bubble volume for I.T.T.C. body, $U=8.7 \mathrm{~m} / \mathrm{s}, \sigma=0.53$.

Figure 6.19 Impulse v. maximum bubble volume for I.T.T.C. body, $U=8.7 \mathrm{~m} / \mathrm{s}$, $\sigma=0.42$.

Figure 6.20 Averaged impulse v. maximum bubble volume for I.T.T.C. body, $U=8.7 \mathrm{~m} / \mathrm{s}, \sigma=0.42$.

Figure 6.21 Average number of peaks v. average maximum bubble volume for bubbles generated on the Schiebe body and I.T.T.C. body. 
Figure 6.22 Numerical calculation of impulse v. maximum bubble volume for bubbles generated on the Schiebe body and I.T.T.C. body.

Figure 6.23 Comparison of theoretically predicted and experimentally measured impulse v. maximum bubble volume relationship for bubbles generated on the Schiebe body and I.T.T.C. body. Experimental data for $\sigma=0.45, U=9 \mathrm{~m} / \mathrm{s}$ for the Sciebe body and $U=8.7 \mathrm{~m} / \mathrm{s}$ for the I.T.T.C. body.

Figure 6.24 Comparison of theoretically predicted and experimentally measured pulse width v. maximum bubble volume relationship for bubbles on the Schiebe body, $U=9 \mathrm{~m} / \mathrm{s}$ and $\sigma=0.45$.

Figure 6.25 Averaged acoustic spectra derived from acoustic pulses generated by bubbles on the Schiebe body at average $U=8.7 \mathrm{~m} / \mathrm{s}$ and average $\sigma=0.45,0.50$, and $0.56 \mathrm{~m} / \mathrm{s}$.

Figure 7.1 Cavitation event rate history for cavitation on the Schiebe body at $U=8.6 \mathrm{~m} / \mathrm{s}$. Operating points were held constant for 10 minutes.

Figure 7.2 Typical distribution of time between cavitation events for cavitation on the Schiebe body. Data taken at $U=8.7 \mathrm{~m} / \mathrm{s}$ and $\sigma=0.45$.

Figure 7.3 Example of four bubble maximum size distributions for a fixed free stream velocity and cavitation number for cavitation on the Schiebe body.

Figure 7.4 Calculated bubble maximum size distribution for cavitation generated on the I.T.T.C. body at $U=9 \mathrm{~m} / \mathrm{s}$ and $\sigma=0.45$ with a free stream nuclei number distribution given by $N\left(R_{O}\right)=10^{-6} R_{O}{ }^{-3.5}$ where $N$ is in $m^{4}$ and $R_{O}$ is in $\mu m$.

Figure 7.5 Measured nuclei number distribution, cavitation event rate, and bubble maximum size distribution for cavitation generated on the I.T.T.C. body at $U=9 \mathrm{~m} / \mathrm{s}$ and $\sigma=0.45$.

Figure 7.6 Calculated event rate and bubble maximum size distribution for cavitation on the I.T.T.C. headform at $U=9 \mathrm{~m} / \mathrm{s}$ and $\sigma=0.45$ using a correlation for the free stream nuclei number distribution derived from the data in Figure 7.5. 
xvii

Figure A.1 Block diagram of the surface electrode system.

Figure A.2 Front and back panel of the probe assembly.

Figure A.3 Circuit board layout of the probe assemmbly.

Figure A.4 Block diagram of the electrode bridge.

Figure A.5 Electrical schematic diagram of the electrode bridge.

Figure A.6 Printed circuit board layout for electrode bridge.

Figure A.7 Component layout for the electrode bridge board.

Figure A.8 Block Diagram of the demodulator.

Figure A.9 Electrical schematic diagram of the demodulator.

Figure A.10 Printed circuit board layout for the demodulator.

Figure A.11 Component layout for the demodulator board.

Figure A.12 Block Diagram of the control system.

Figure A.13 Electrical schematic diagram of the bus interface.

Figure A.14 Electrical schematic diagram of the system interface.

Figure A.15 Electrical schematic diagram of the zeroing voltage circuit.

Figure A.16 Electrical schematic diagram of the reference voltage circuit.

Figure A.17 Electrical schematic diagram of the carrier generator.

Figure A.18 Digital codes for the controller system.

Figure B.1 Schematic diagram of the attached cavity experiment.

Figure B.2 Examples of attached cavities for $\sigma=0.40,0.38,0.36$ and 0.34 at $U=9 \mathrm{~m} / \mathrm{s}$.

Figure B.3 Cavity length v. cavitation number for various tunnel velocities.

Figure B.4 Mean and standard deviation of the electrode signal v. cavitation 
xviii

number. Vertical scale is arbitrary.

Figure B.5 Electrode signal spectra for $\sigma=0.40$ and 0.34 at $U=9 \mathrm{~m} / \mathrm{s}$. Vertical scale is arbitrary.

Figure B.6 One possible mode of cavity oscillation.

Figure B.7 Acoustic spectra for $\sigma=0.40$ and 0.32 at $U=9 \mathrm{~m} / \mathrm{s}$ and for $\sigma=1.0$ at $9.5 \mathrm{~m} / \mathrm{s}$. Vertical scale is arbitrary. 


\section{CHAPTER 1}

\section{Introduction}

\subsection{Current Knowledge of Bubble Cavitation}

Travelling bubble cavitation occurs when small bubbles in a fluid experience pressures below the liquid vapor pressure. These small bubbles, or nuclei, grow to become cavitation bubbles, and if the cavitation bubbles are swept into regions of high pressure they will collapse, often producing undesirable effects. Cavitation bubbles may significantly alter the flow, and collapsing bubbles may cause damage to solid-boundary surfaces and produce undesirable acoustic emissions. Designs of ship propellers, hydrofoils, and turbomachinery must consider the phenomenon of bubble cavitation.

The dynamics and acoustics of travelling bubble cavitation have been extensively studied both experimentally and theoretically since Rayleigh's analysis of bubble dynamics (Rayleigh (1917)). Knapp and Hollander's (1948) experimental observations of bubble cavitation and Plesset's (1949) analysis provided the basis for much of the modern understanding of bubble dynamics, and subsequent researchers have extended the theoretical analysis of cavitation bubbles to include most physical aspects of the flow. A complete review of this topic may be found in Cavitation by Knapp, Daily, and Hammitt (1970).

Yet the actual behavior of individual cavitation bubbles may radically depart from that predicted by theoretical considerations. It has been known for some time that cavitation bubbles generated near surfaces are not generally spherical, as often assumed by theory, but hemispherical caps (Knapp and Hollander (1948) and Parkin (1952)), and a cavitation bubble collapsing near a solid boundary may produce a

microjet of fluid, which has been speculated to cause surface cavitation damage (Benjamin and Ellis (1966), Plesset and Chapman (1970), Lauterborn and Bolle (1975), Kimoto (1987) and, for a review, Blake and Gibson (1987)). The complex 
shapes that travelling bubbles assume will clearly be influenced by macroscopic flow phenomena such as pressure gradients, boundary layers, separation, and turbulence. Researchers have attempted to study these effects by observing cavitation bubbles induced in a venturi (Kling and Hammitt (1972)) or above a surface (Chahine, Courbiere, and Garnaud (1979), van der Meulen (1989)). Yet detailed, systematic studies of hydrodynamically-produced cavitation bubbles are almost non-existent. The random nature of naturally occurring cavitation is the primary reason why investigators have focused on integral measurements in their study of cavitating flows, leaving the detailed behavior of individual cavitation bubbles unexamined.

\subsection{Current Knowledge of Cavitation Noise}

Analyses of cavitation noise have generally been based on the theoretical behavior of single bubbles following the work of Fitzpatrick and Strasberg (1956), which is derived from the Rayleigh-Plesset equation and therefore has all the same issues of applicablity. From this data base, researchers have synthesized the acoustic emission from cavitating flows with multiple events, and much of this work is presented in the excellent review by Blake (1986). Many experiments have attempted to extract the actual behavior of individual bubbles from the integral measurement of the noise produced by cavitation and examples of these studies are Mellon (1956), Blake, Wolpert, and Geib (1977), Hamilton (1981), Hamilton, Thompson, and Billet (1982), and Marboe, Billet, and Thompson (1986). Although trends are seen in the measured spectra which may be related to theoretical predictions, the difficulty of obtaining free field acoustic spectra in the confines of most water tunnels has always made interpretation of experimental spectra problematic.

Researchers have attempted to treat the cavitation as a stochastic process. The spectral emission of a cavitating flow will depend not only on the noise produced by single bubbles but also on the cavitation rate and event statistics (Morozov (1969) and Baiter (1986)). Furthermore, cavitation noise scaling will be significantly influenced by changes in the cavitation event rate. As the number of cavitation 
events increase, bubble interactions will affect individual bubble volume histories and their acoustic emission (e.g., Morch (1982), Arakeri and Shangumanathan (1985), and d'Agostino, Brennen, and Acosta (1988)). Analyses of multiple bubble effects rest upon a knowledge of the nuclei distribution in the flow and the dynamics causing them to cavitate.

The effect of nuclei number distribution on the total cavitation process is poorly understood, and this is due largely to the difficulty of accurately measuring this quantity. In fact, most cavitation studies neglect to include any measure of the nuclei number distribution. The number and size distribution of cavitation bubbles, and the resulting noise emission, can vary substantially over the course of an experiment, even at a nominally fixed operating point. Although the mean cavitation event rate may be approximately determined by the acoustic pulse rate (Marboe, Billet, and Thompson (1986)), cavitation bubble size distributions have only been determined in very rough form (Baiter (1974) and Meyer, Billet, and Holl (1989)). Although knowledge of the cavitation rate and bubble size distribution is essential, no simple method has been found to count and measure cavitation bubbles.

The above observations indicate a need to study the dynamics and acoustic emission of individual cavitation bubbles. A method of detecting and measuring cavitation bubbles was needed, and this thesis presents data obtained through the use of a new electrical probe developed for this purpose. Also, the dynamic response of the probe permits statistical analysis of the cavitating flow. With this new instrument experiments were performed to study individual cavitation events and their statistics in an attempt to address the above issues.

\subsection{Outline of Research}

Cavitation bubbles were observed on two standard axisymmetric bodies which were installed in a recirculating water tunnel. A description of the experimental 
equipment is presented in Chapter 2, and Chapter 3 provides a detailed description of the surface impornnce probe.

Cavitation bubbles that occur naturally in flows around bodies were examined to determine the effect of the viscous flow (boundary layers, separation, transition) on the growth and collapse of the bubbles and the noise produced upon collapse. Individual bubbles were detected and photographed, and their acoustic emission was recorded. Two different axisymmetric headforms, the Schiebe and I.T.T.C. body, were used to generate cavitation, and the growth and collapse of the bubbles were related to the flow around each headform. These results are described in Chapter 4.

The dynamics and acoustics of single cavitation bubbles have been studied by many researchers, and a summary of this analysis along with the results of numerical integrations of the Rayleigh-Plesset equation are presented in Chapter 5. Experimental measurement of single bubble acoustic emissions are compared with these theoretical predictions in Chapter 6 .

Cavitation event statistics and bubble maximum size distributions were measured and compared with the experimentally determined nuclei number distribution. These results are compared to the predictions of a simple model in Chapter 7. Finally, the above results are discussed in Chapter 8 . 


\section{CHAPTER 2}

\section{Experimental Equipment}

\subsection{Water Tunnel}

The experiments were conducted in the Caltech Low Turbulence Water Tunnel (LTWT) whose schematic is presented in Figure 2.1. A full description of the facility is presented by Gates (1977), but a brief description of the main features are presented here.

The LTWT test section is $2.54 \mathrm{~m}$ long and has a rectangular test section that expands from $0.305 m$ by $0.305 m$ at the entrance to $0.356 m$ by $0.305 m$ at the exit. The upstream settling tank has a cross section of $1.22 \mathrm{~m}$ by $1.22 \mathrm{~m}$ resulting in a contraction ratio of $16: 1$. This settling tank has two honeycombs and three damping screens that reduce the test section turbulence level to 0.04 percent.

The LTWT has a maximum velocity of approximately $10 \mathrm{~m} / \mathrm{s}$. A vacuum may be applied to the tunnel to reduce the test section static pressure to a minimum of approximately $14 k P a$. The air content of the tunnel is controlled through air injection and deaeration, and the air content may vary from 3 to 15ppm. This quantity is measured with a Van- Slyke blood gas apparatus. The water temperature was approximately $20^{\circ} \mathrm{C}$.

For all experiments, the test section free stream velocity was set and the tunnel static pressure lowered until the desired cavitation number was reached. The operating air content was generally between 6 to $8 \mathrm{ppm}$, and the tunnel water was well filtered.

\subsection{Test Bodies}

Two standard axisymmetric headforms were used in the present experiments. The first was a Schiebe headform (Figure 2.2a) with a truncated diameter of $5.08 \mathrm{~cm}$. (Gates et. al. (1979)); the second, which has a modified ellipsoidal shape and a 
diameter of $5.59 \mathrm{~cm}$, is known as the I.T.T.C. headform (Figure 2.2b) (Lindgren and Johnsson (1966)). The axisymmetric headforms were mounted on a two bladed sting with a nominally zero degree yaw. Figure 2.3 shows a schematic diagram of the experimental setup, and Figure 2.4 presents a photograph of the strut assembly mounted in the LTWT. The headforms were fabricated out of lucite, a material whose acoustic impedance closely matches that of water.

\subsection{Hydrophone System}

\subsubsection{Hydrophone Setup}

The hollow interior of both bodies was filled with water in which a hydrophone was placed (see Figure 2.4). This configuration created a nominally reflection free acoustic path from the surface of the headform to the hydrophone. The acoustic measurements were made with this hydrophone, an ITC-1042 which has a relatively flat response out to $80 \mathrm{kHz}$ (Figure 2.5). Except for ultralow frequencies ( $\ll 1 \mathrm{~Hz}$ ), the hydrophone signal was not filtered. All acoustic signals were digitized at a sampling rate of $1 \mathrm{MHz}$. Because of the relatively good acoustic impedance match between lucite and water, the interior hydrophone allows the noise generated by the cavitation bubbles to reach the hydrophone relatively undistorted; reflected acoustic signals from other parts of the water tunnel only make their appearance after the important initial signal has been recorded.

\subsubsection{Hydrophone Calibration}

The steady-state, far-field calibration curve of the ITC-1042 hydrophone was used to convert the hydrophone voltage signal to pressure. This raises several issues. First, the hydrophone was calibrated for signals generated in the far field, but the location of the hydrophone in the experiment is within one hydrophone diameter $(35 \mathrm{~mm})$ of the noise source. Since the acoustic path between the bubble and the hydrophone is not perfectly transparent, acoustic focusing and scattering may have occurred. A qualitative attempt to determine the effect of the hydrophone location 
was performed. A second identical hydrophone was placed in an acoustic tank at a distance approximately 10 hydrophone diameters away from the bubble noise source, and a nominally reflection free acoustic path was provided through a tunnel window made of lucite." Both hydrophones were used to record the emission of a single cavitation event using identical electrical conditioning systems, and the two traces were compared. Figure 2.6 provides an example of two such signals. The two signals are nominally the same shape, although there is the expected attenuation of signal recorded from the far hydrophone. This attenuation is on the order of 10:1, which corresponds to the approximate ratio of the two hydrophone path lengths. The signal reaching the far hydrohone has also lost some high frequency content, which is likely due to dispersive effects in the long acoustic path, such as small bubbles. Examination of many such signals lends confidence to the conclusion that signals recorded by the internal hydrophone were not severely altered by the hydrophone placement.

Secondly, the relationship used to convert the voltage output of the hydrophone to pressures was arrived at through a steady-state calibration. The signals measured in this experiment, however, are transient. The response charateristics of the hydrophone in the time domain are not known, and the steady-state calibration data is used only as a substitute. Further calibration of the hydrophone system is necessary to adequately charaterize the time response.

\subsection{Nuclei Counter}

The free stream nuclei number distribution of the upstream fluid was measured using a in-line pulsed holography system described by Katz (1981). With this method, a three-dimensional image of a sample volume of tunnel water is recorded. Small bubbles or nuclei recorded in the image may then be counted and sized to determine the sample nuclei number distribution. A sample volume of $322 \mathrm{~cm}^{3}$ was analyzed. Holography permits bubbles to be distinguished from dirt particles, and the smallest detectable nucleus is approximately $20 \mu \mathrm{m}$ in diameter. 


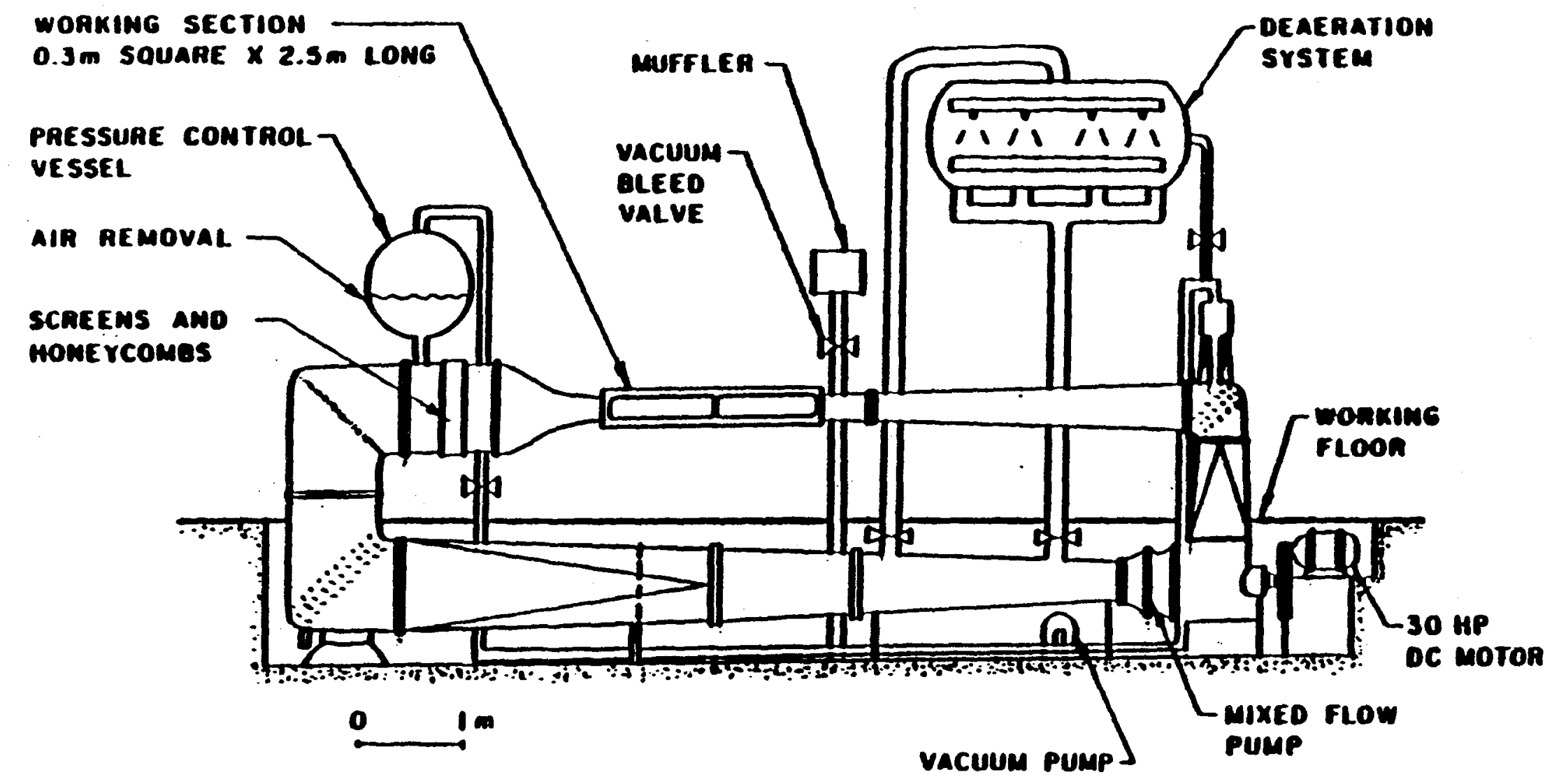

$\infty$

Figure 2.1 Schematic diagram of the Caltech Low Turbulence Water Tunnel. 


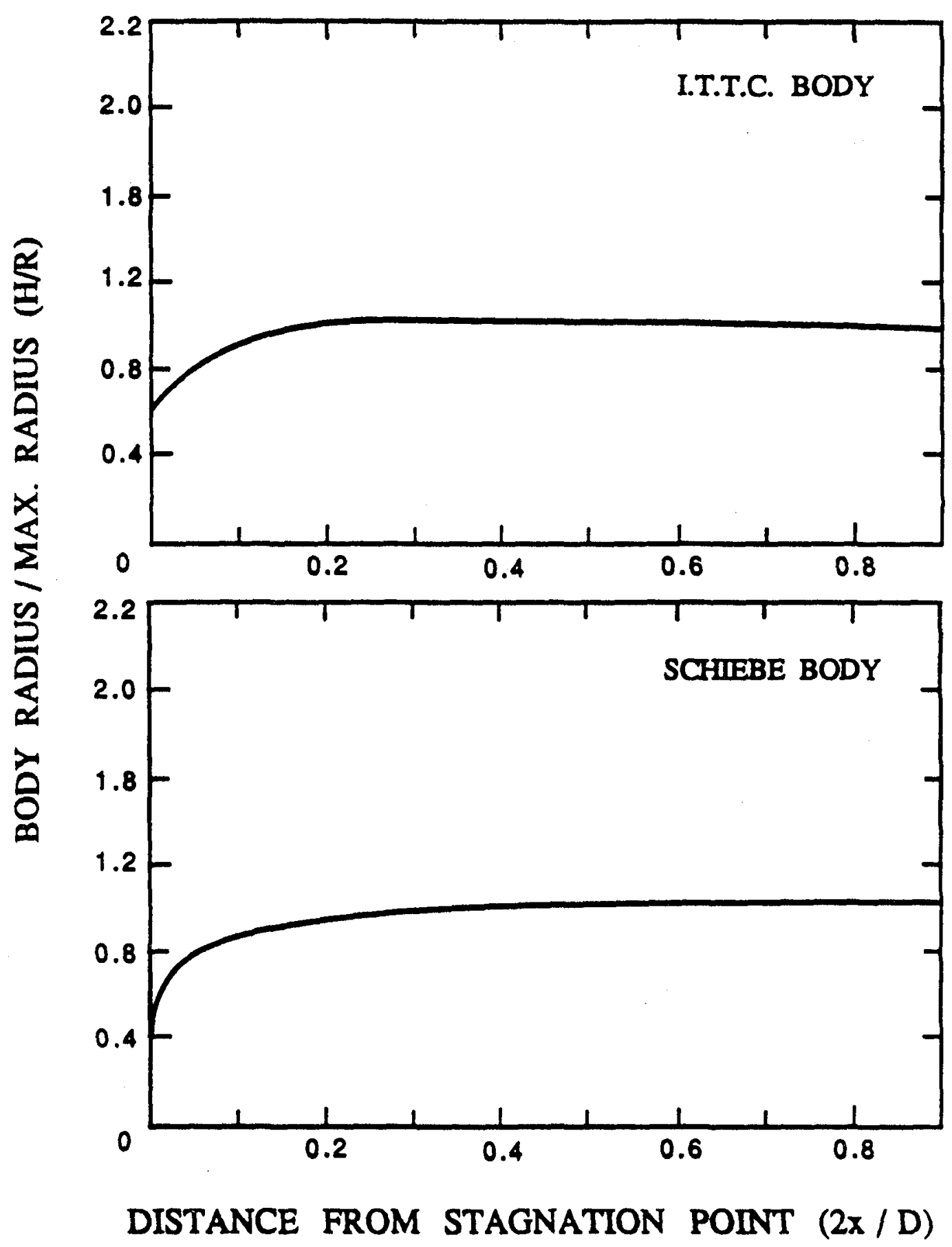

Figure 2.2 Profile of the Schiebe and I.T.T.C. headform. 

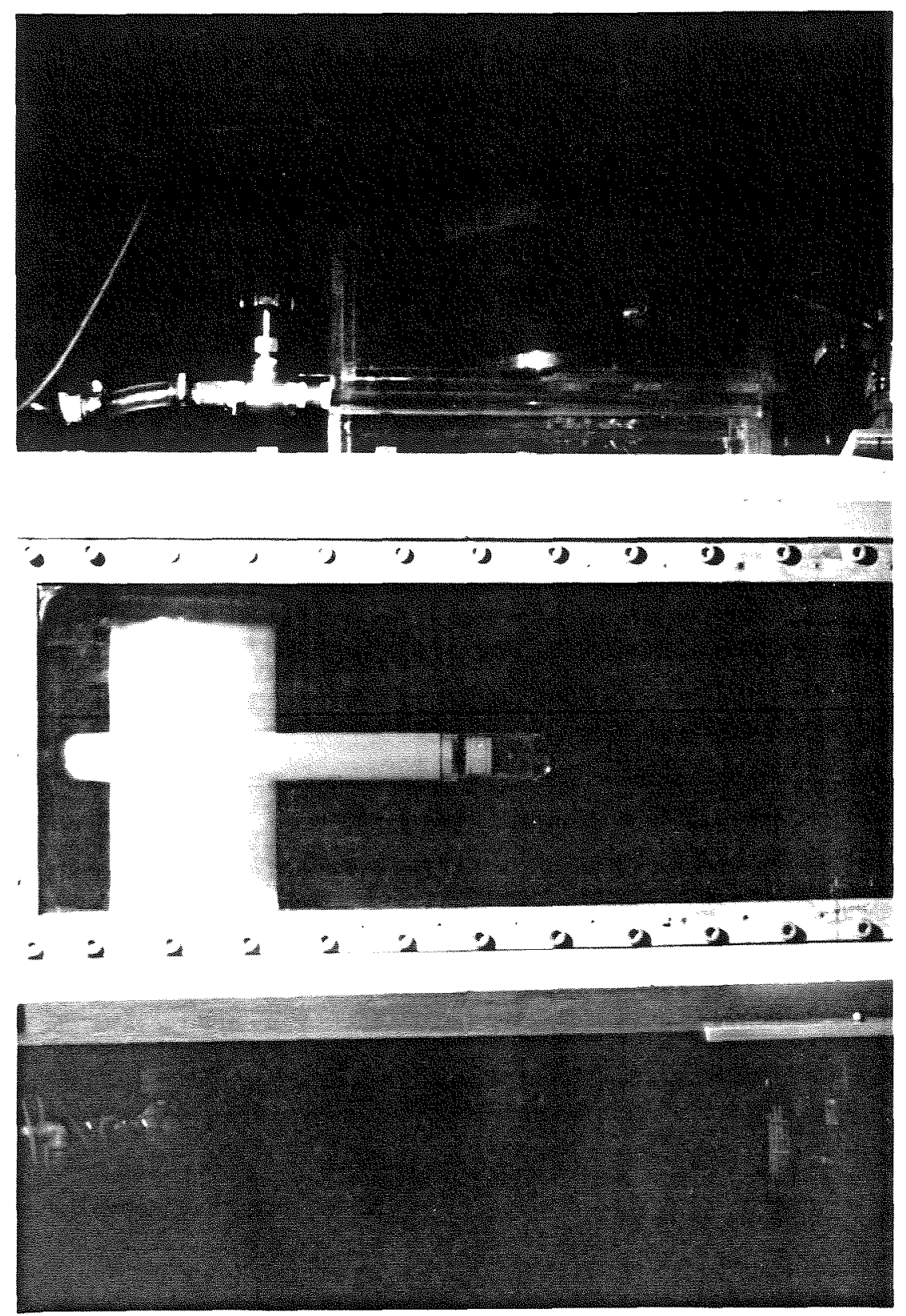

Figure 2.3 Photograph of the I.T.T.C. headform mounted in the L.T.W.T. test section on the two bladed sting. 


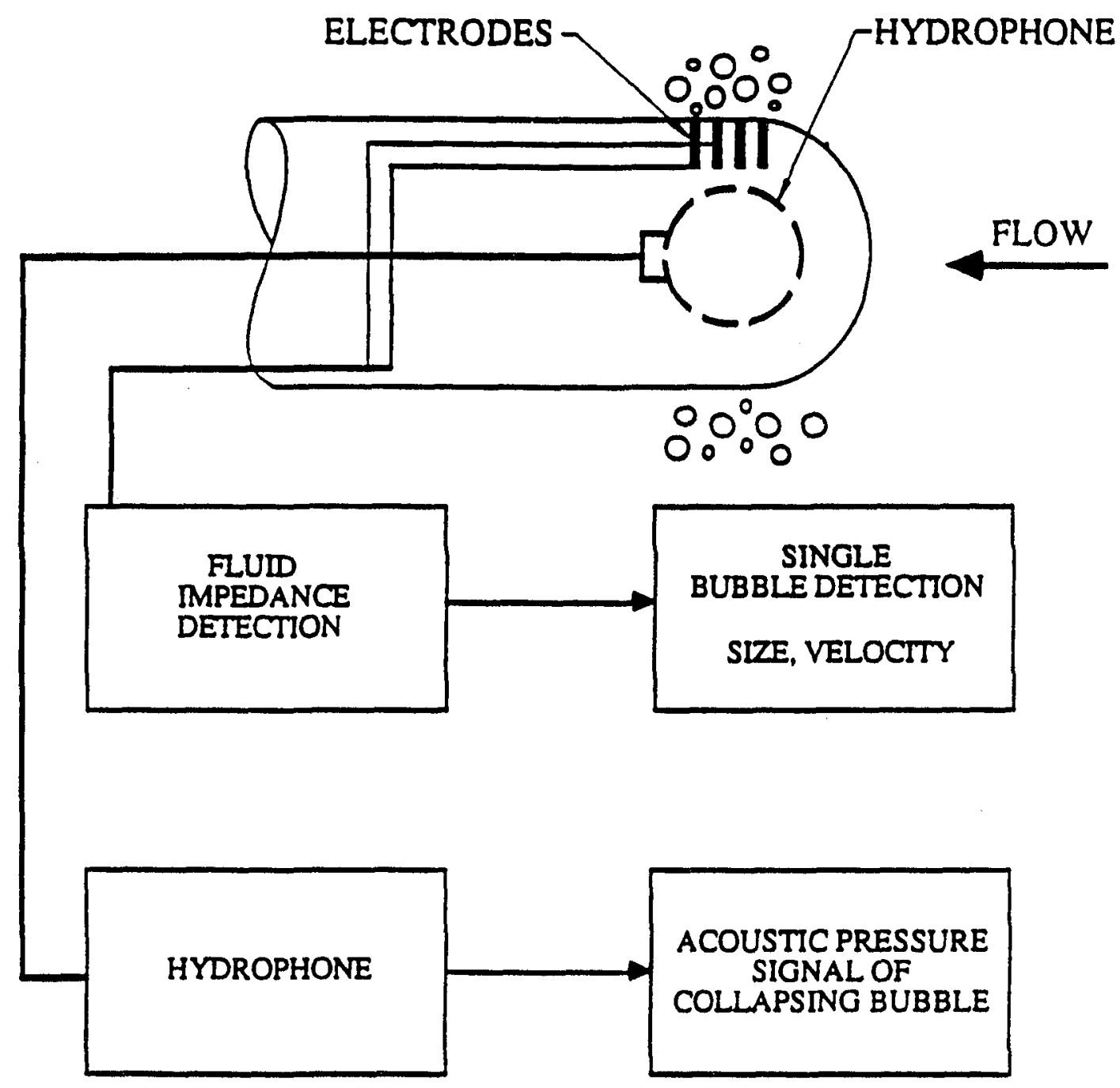

Figure 2.4 Schematic diagram of the bubble cavitation experiment. 


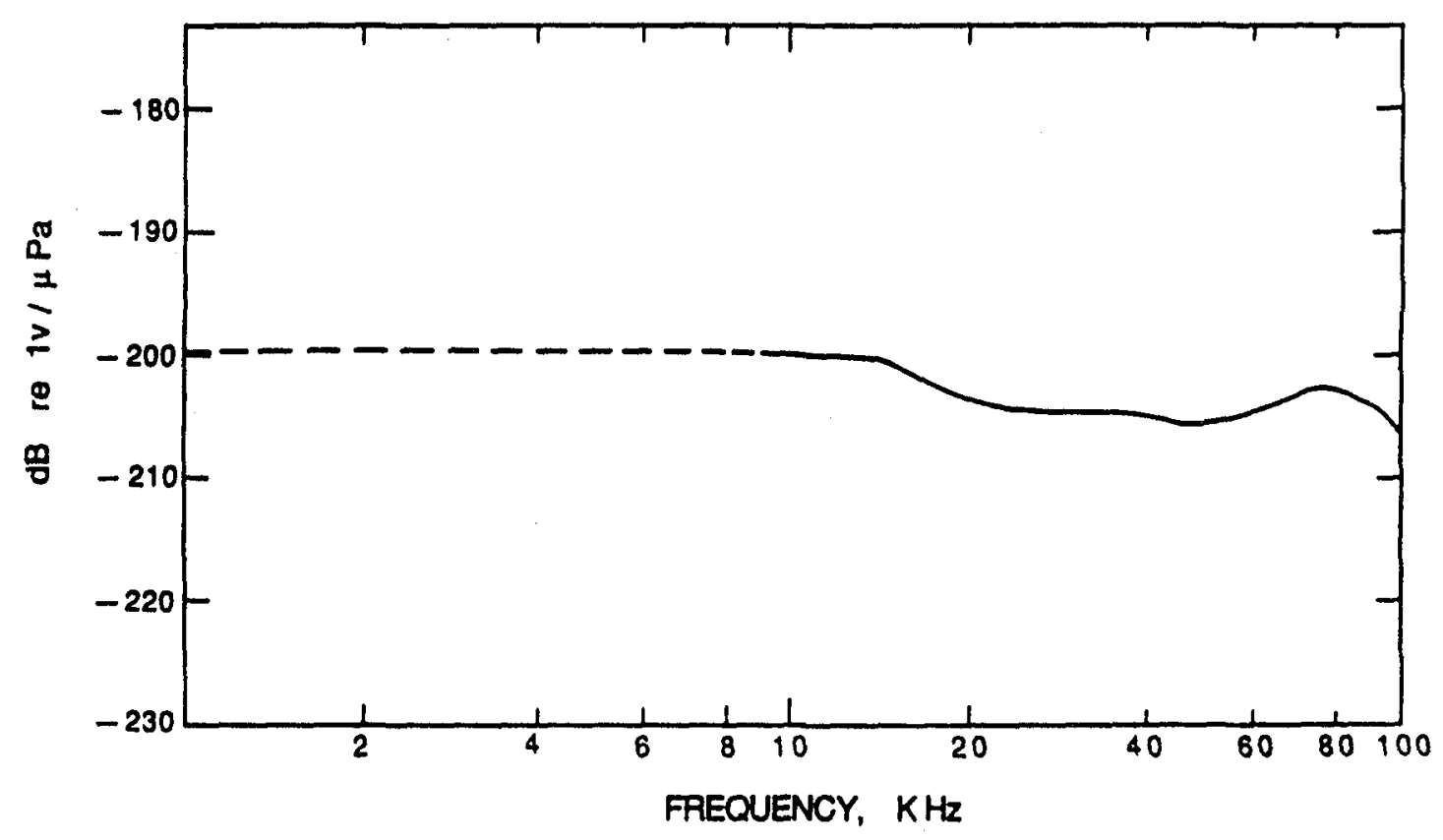

Figure 2.5 Receiving response of ITC-1042 hydrophone. 


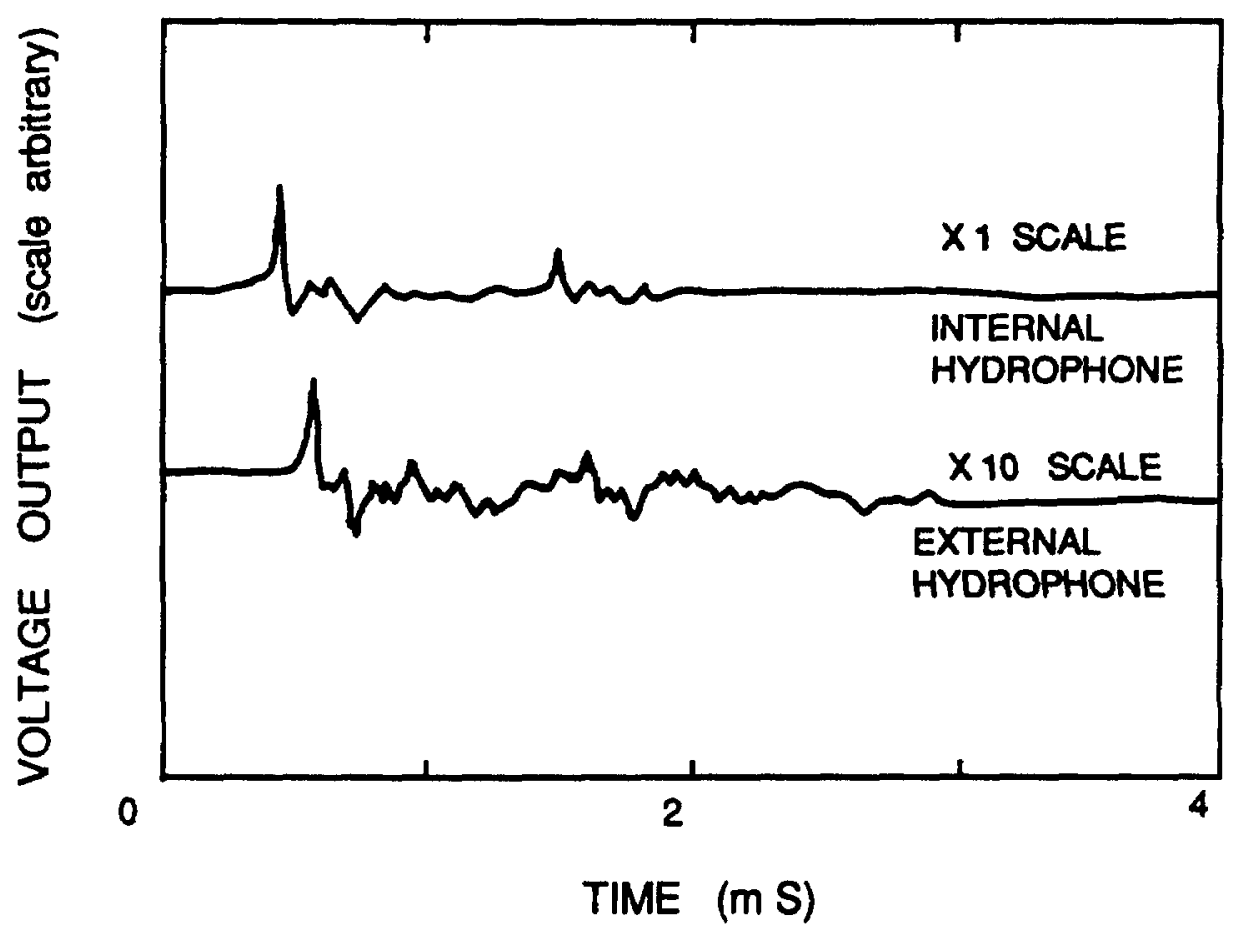

Figure 2.6 Dual signal trace from two ITC-1042 hydrophones. The top signal is from the internal hydrophopne, the bottom signal is from the external hydrophone. 


\section{CHAPTER 3}

\section{Surface Electrode Probe}

\subsection{Introduction}

In addition to the hydrophone, each headform was provided with novel equipment developed from instrumentation that had previously been used to measure volume fractions in multiphase flows (Bernier (1981)). This instrumentation consisted of a series of electrodes arrayed on the headform surface, which were used to detect and measure individual cavitation bubbles.

\subsection{Principle of Operation}

A pattern of alternating electric potentials is applied to the electrodes and the electric current from each is monitored. When a bubble passes over one of the electrodes the impedance of the local conducting medium is changed, causing a change in current from the electrode which is detected and recorded. This change is related to the position and volume of the bubble. Consequently, the electrode array allows passive detection and monitoring of individual cavitation bubbles.

The bulk impedance of the fluid is complex but may be modeled as a combination of capacitive and resistive elements if the electric field frequency is high enough to overcome polarization effects at the electrode-fluid interface (Olsen (1967)). Pure water is a poor electrical conductor, but the water in the LTWT was highly conductive due to chemical additives applied to prevent corrosion. Also, by minimizing the electrode current, the electrical energy dissipated in the fluid will be quite small, making the probe almost completely passive. A detailed technical description of the probe is presented in Appendix A.

One specific geometry consisted of electrode patches arrayed in the flow direction to cover the major extent of the cavitating region. Another consisted of electrodes that encircled the entire circumference of the headform in the region of maximum bubble growth. For all geometries the electrode thickness in the direction 
of bubble motion was on the order of $0.1 \mathrm{~cm}$. The electrodes were fabricated using electrically conducting epoxy which could be machined and polished along with the lucite.

\subsection{Electrode Geometry}

\subsubsection{Patch Electrodes}

The "patch" and "circular" electrode geometries, as they will be called, were used for different purposes. Signals from the patch electrodes indicated cavitation at a well defined location on the headform, and, by electronically triggering flash

photography, simultaneous plan and profile photographs of individual bubbles were taken at a prescribed moment in the bubble history. Thus, a whole series of bubbles could be inspected at the same point in their trajectory. Furthermore, by simultaneously recording the acoustic signal from the hydrophone, one could correlate the noise with the geometry of the bubbles.

Both the Schiebe and the I.T.T.C. bodies were instrumented with sixteen patch electrodes positioned under the entire region of the longest bubble trajectory. The Schiebe body had patches over the range of $s / D=0.38$ to 0.98 (Figures 3.1 and 3.2) where $\mathrm{s}$ is the streamwise coordinate measured along the surface of the body from the stagnation point. The I.T.T.C. headform had patches over the range of $s / D=0.34$ to 1.20 (Figures (3.3 and 3.4)). A pattern of alternating voltages was applied to each electrode, and an example of the electrode output is shown in Figure 3.5. By triggering the camera with each electrode, detailed photographs of the bubbles could be consistently obtained at each electrode location. As the bubbles collapsed, their volume decreased making the bubbles difficult to detect with the electrode system; therefore, a digital delay unit was used to determine the details of the bubble collapse: a bubble could be detected just prior to collapse, and the delay could be adjusted to trigger the flash unit during all phases of the collapse and rebound. 


\subsubsection{Circular Electrodes}

The circular geometry was used to detect the occurrence of every cavitation bubble at a particular location on the headform. This position was chosen to be near the location of maximum bubble volume, and for relatively moderate event rates only one bubble would occur over the electrode at any given time.

Three electrodes were used in this geometry. The center electrode was positioned at the average point of bubble maximum volume and was used to detect the bubbles. The two electrodes bracketing the measuring electrode provided a sink for the current generated by the measuring electrode. By using two shielding electrodes, the symmetry of the center electrode was preserved. For the Schiebe body, the electrodes were located at $s / D=0.61,0.65$, and 0.69 (Figures 3.6 and 3.7), and for the I.T.T.C. headform they were located at $s / D=0.38,0.40$, and 0.69 (Figures 3.8 and 3.9$)$.

\subsection{Calibration}

Because almost all the cavitation bubbles maintain the same distance above the electrodes (this will be discussed below), the output of the circular electrode system is directly proportional to the area covered by the bubble, and the peak of the signal is proportional to the major diameter of the bubble (Figure 3.10). This system was calibrated photographically and found to be quite linear. A sample calibration curve is included in Figure 3.11.

The location of the measuring electrode was chosen to be close to the point of maximum volume for most bubbles, although the exact location of bubble maximum volume will vary for different bubbles and cavitation numbers. Photographic observations indicated that bubbles maintained approximately their maximum volume for distances at least three times the electrode width, although for cavitation numbers near inception, some smaller bubbles may reach their maximum volume before reaching the electrode. 
The volume of the bubbles can be determined from a measure of the base diameter of the bubble if the bubble shapes are assumed self similar. A functional relationship between the base diameter, $D_{B}$, and the bubble volume, $V_{B}$, was derived through the photographic study of many individual bubbles. For the Schiebe body this formula was

$$
V_{B} \approx 0.095 D_{B}^{3}
$$

and for the I.T.T.C. body,

$$
V_{B} \approx 0.125 D_{B}^{3}
$$

These relationships were used to convert the electrode measurements to bubble volumes.

\subsection{Post Signal Processing}

The output signal of the patch electrode probe was used to trigger the camera and acoustic data acquisition systems, and a schematic diagram of this system is shown in Figure 3.12. Because bubbles would often graze the patch electrodes, the voltage output of these electrodes was not used quantitatively, but the circular

electrode signal could be calibrated to provide a measure of the bubble size, as described above.

Two general experiments were performed with the circular electrode system. The first involved the measurement event statistics and bubble maximum size distributions (Figure 3.13). A peak detector was used to isolate the maximum of the electrode signal that represented the bubble maximum volume, and the time between events was measured with a voltage ramp generator whose output was proportional to the time delay. The peak detector triggered a digital data acquisition system to record both signal voltages. This system easily detected and measured all cavitation events, and the results could be displayed in real time.

Acoustic emission of individual cavitation bubbles was also analyzed (Figure 3.14). The output of the peak detector was used to trigger the acoustic data 
acquisition system. In this way, the acoustic emission of a detected bubble could be recorded along with the bubble size. Analysis of the acoustic signal occurred immediately after data acquisition and would take approximately one-half second. While this data reduction was taking place, the bubble size measurement system was paused with a lock out system. A photograph of the electronic equipment is shown in Figure 3.15.

\subsection{Conclusion}

The electrode system provides a simple, robust, and non-intrusive method of detecting and measuring individual travelling cavitation bubbles. By varying the electrode geometry, different aspects of bubble cavitation may be studied. Furthermore, the dynamic response of the electrode system allows for real time measurement of all cavitation events. The data obtained with this probe will be presented below.

The electrode system may also be used to study the size and fluctuations of attached cavitation. A preliminary study of attached cavitation using the previously mentioned headforms was conducted, and the results are presented in Appendix B. 


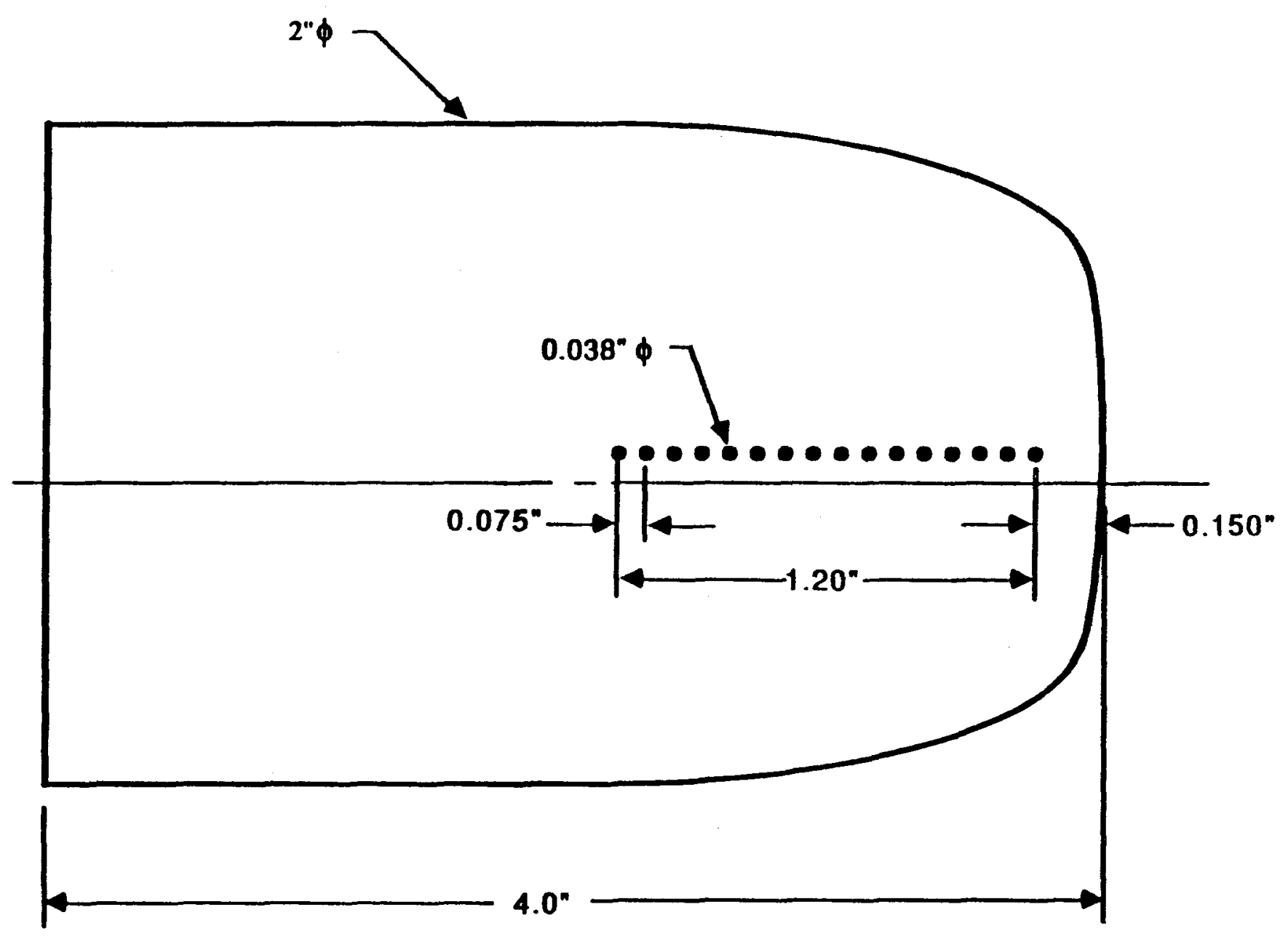

Figure 3.1 Schematic diagram of Schiebe headform with patch electrodes. 


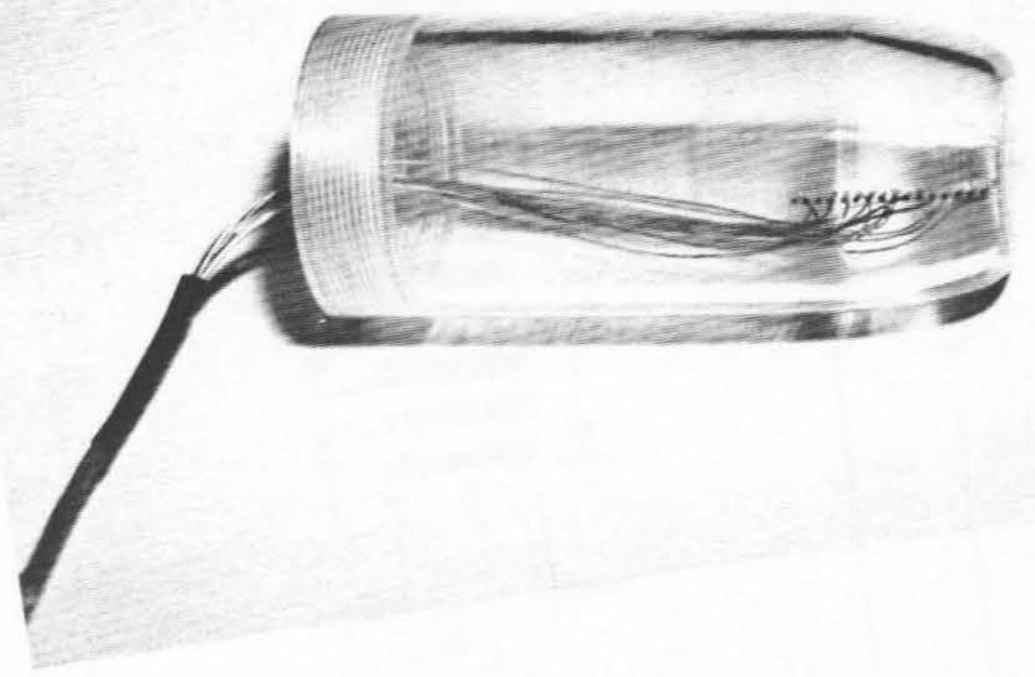

Tigure 3.2 Scheibe headform with patch electrodes. 


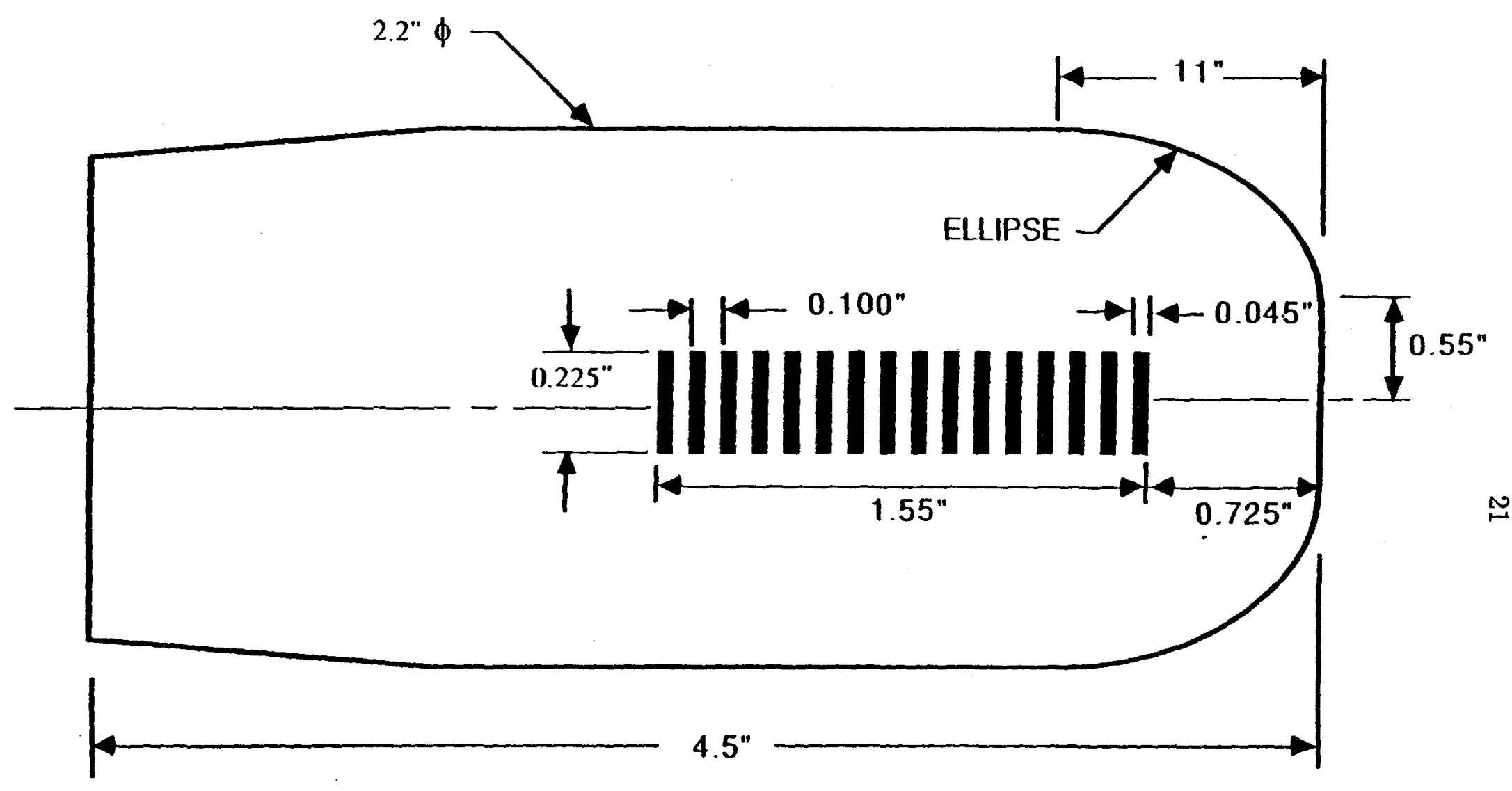

Figure 3.3 Schematic diagram of the I.T.T.C. headform with patch electrodes. 


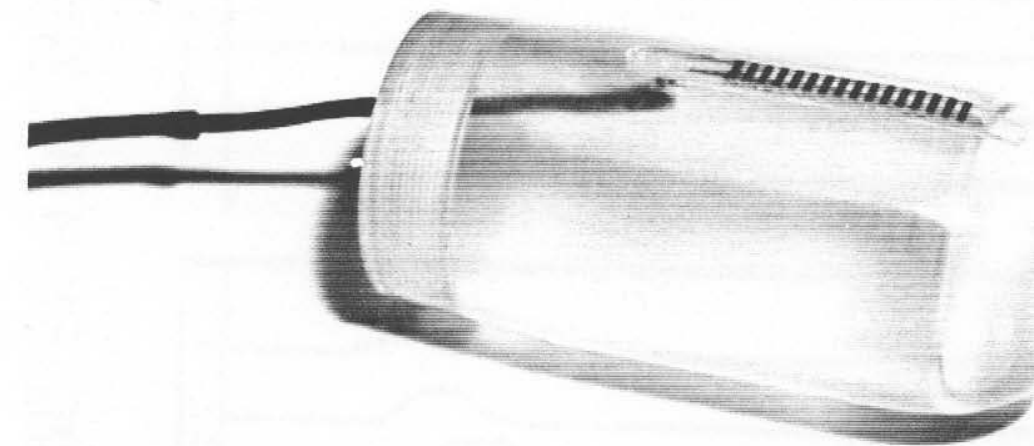

Figure 3.4 I.T.T.C. headform with patch electrodes. 


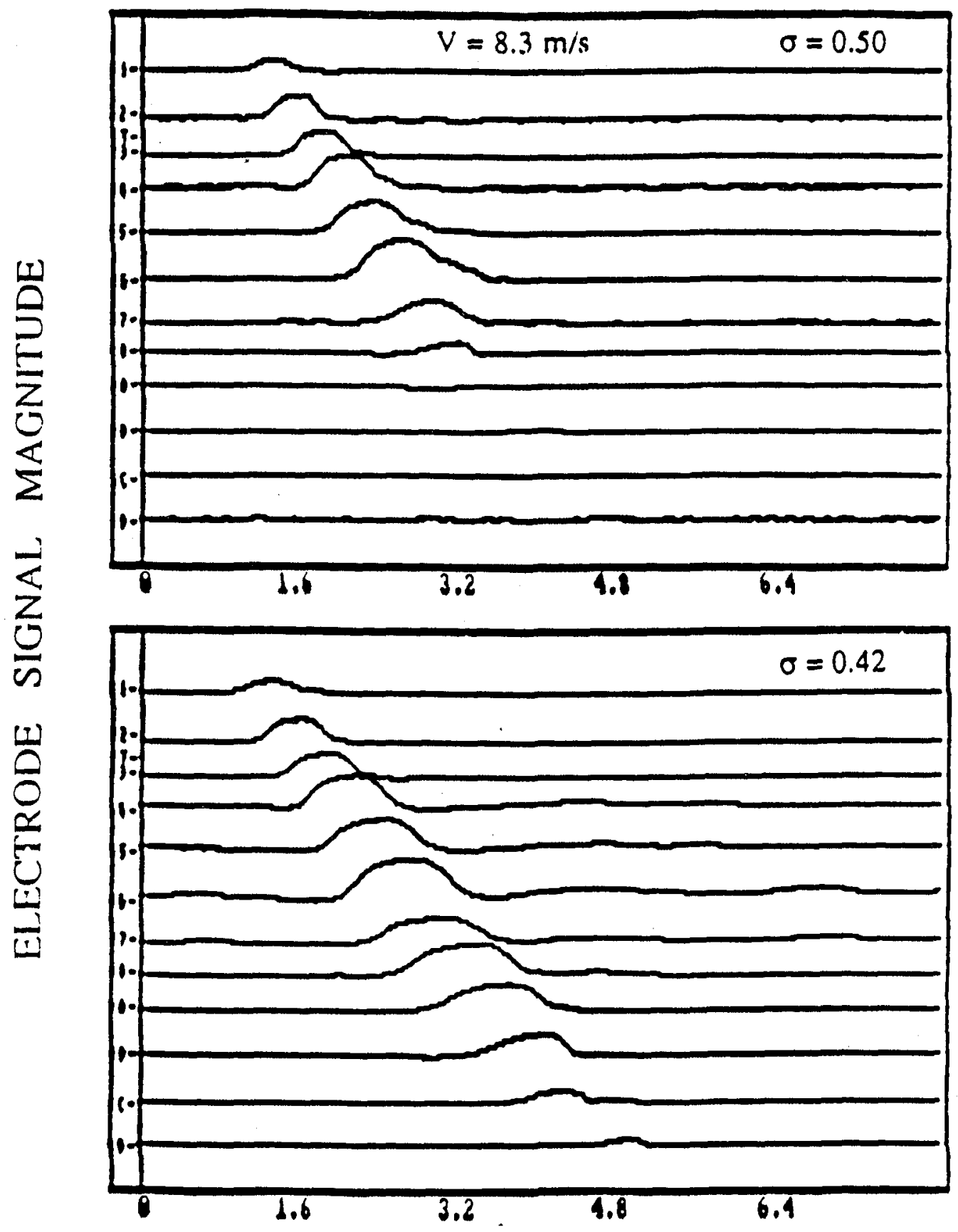

TIME, m sec.

Figure 3.5 Example probe output signal for patch electrodes. Bubbles generated on the I.T.T.C. headform at $\sigma=0.50$ and $\sigma=0.42$ at $U=8.3 \mathrm{~m} / \mathrm{s}$. 


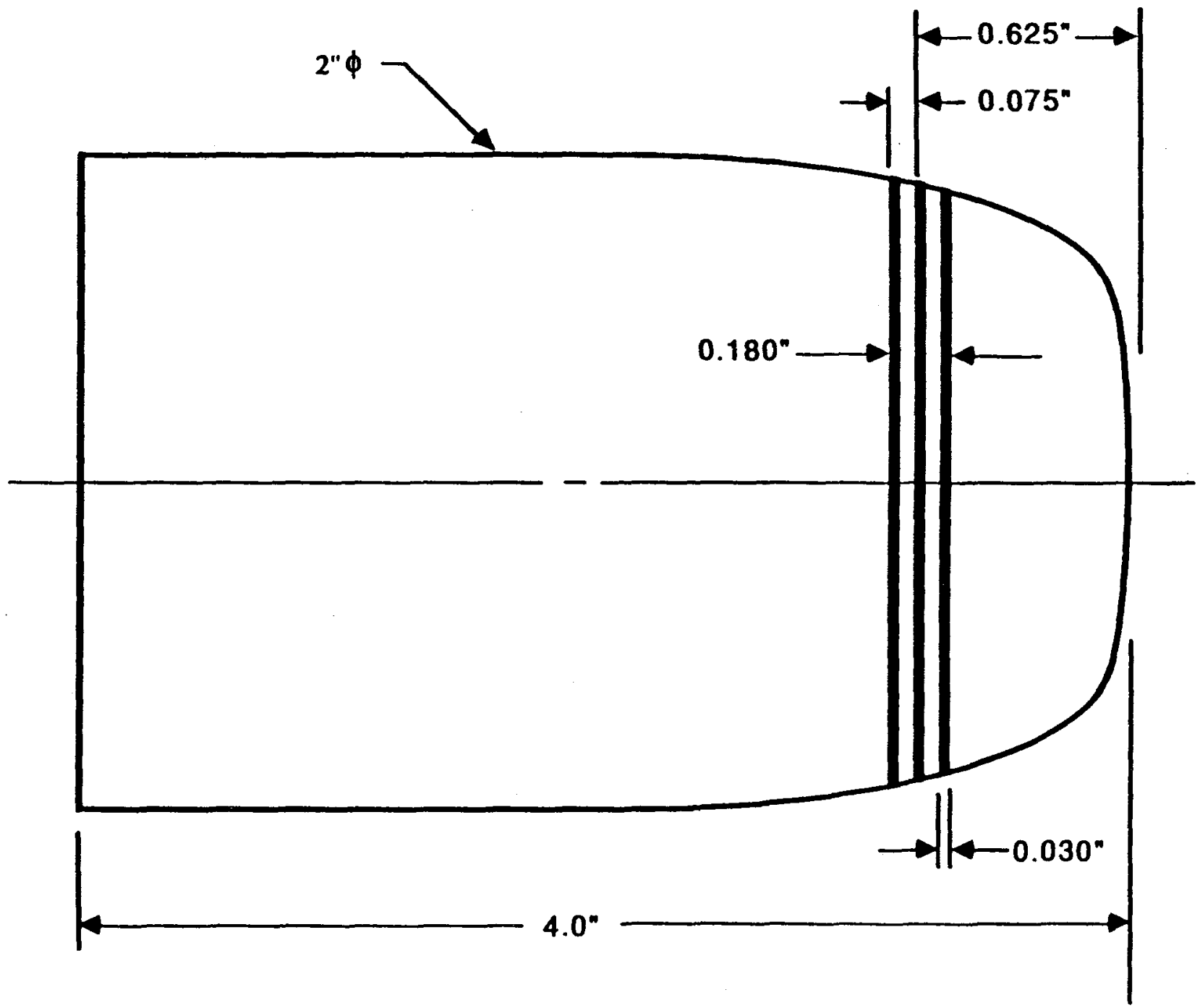

$\stackrel{N}{\oplus}$

Figure 3.6 Schematic diagram of Schiebe headform with circular electrodes. 

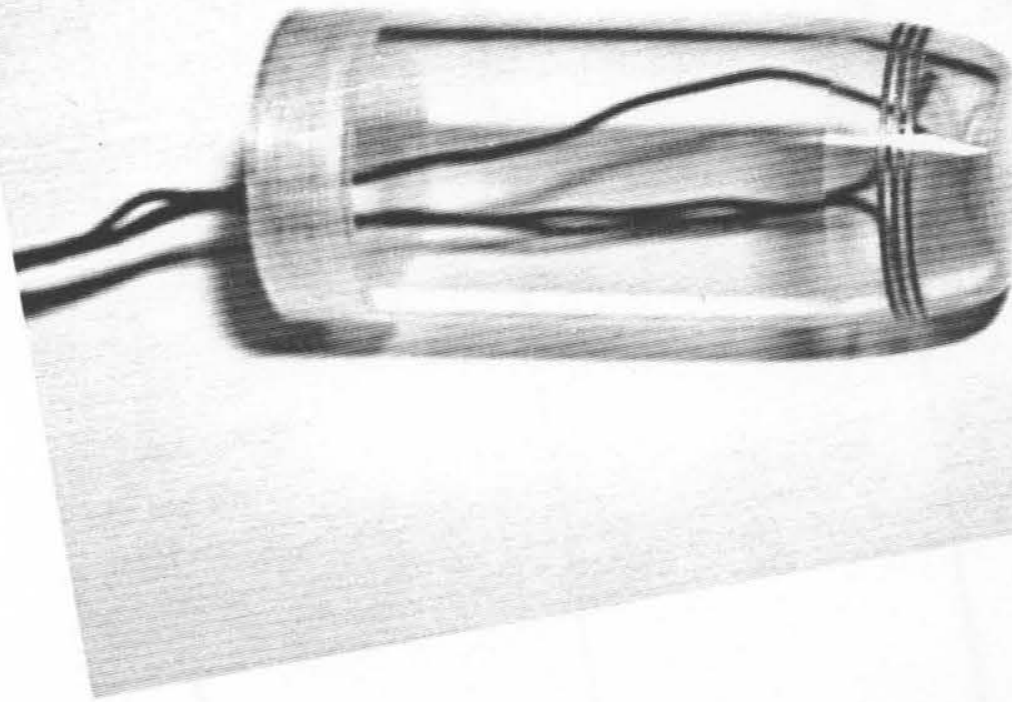

Figure 3.7

adform with circular electrodes. 


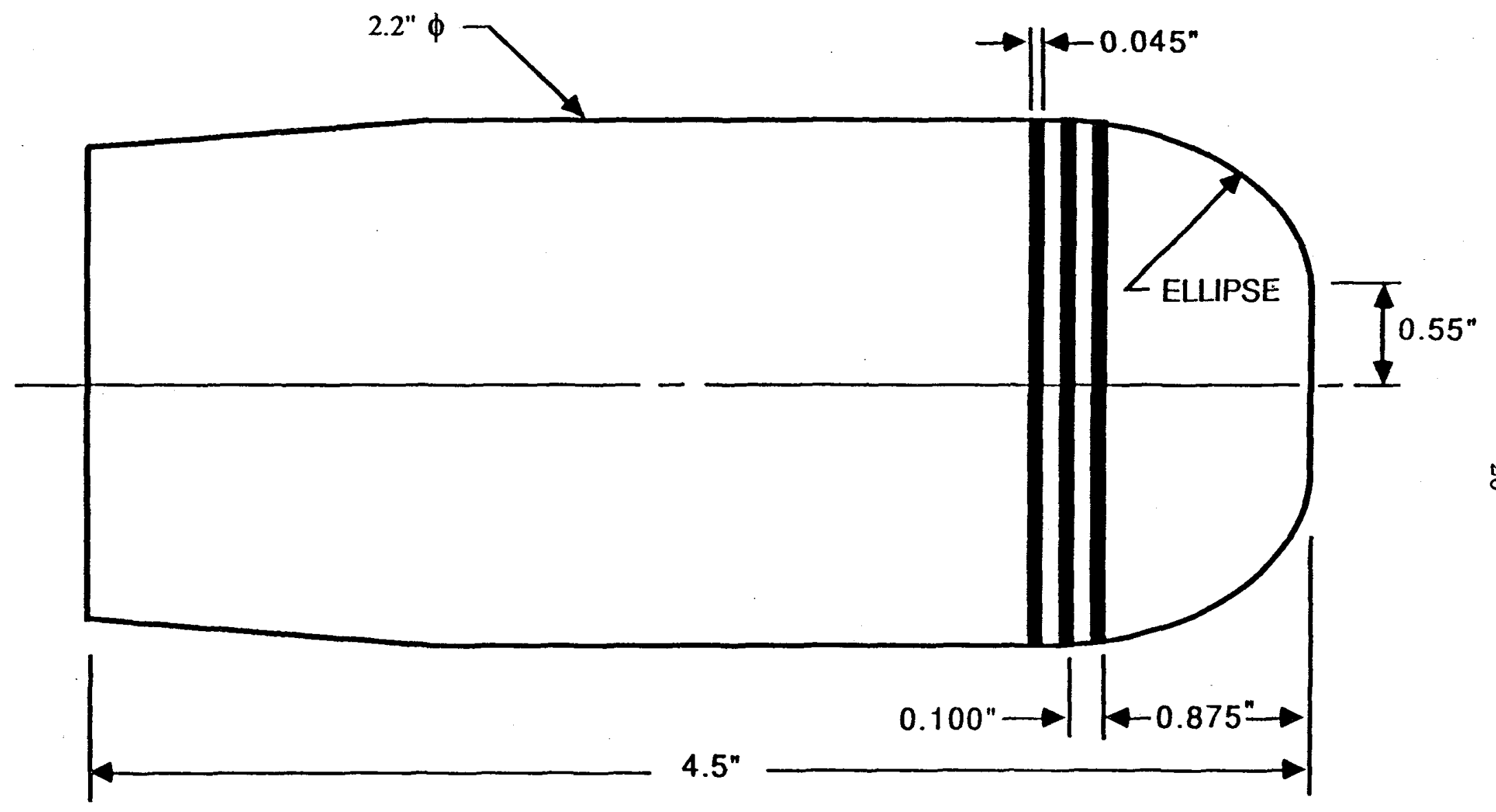

Figure 3.8 Schematic diagram of the I.T.T.C. headform with circular electrodes. 


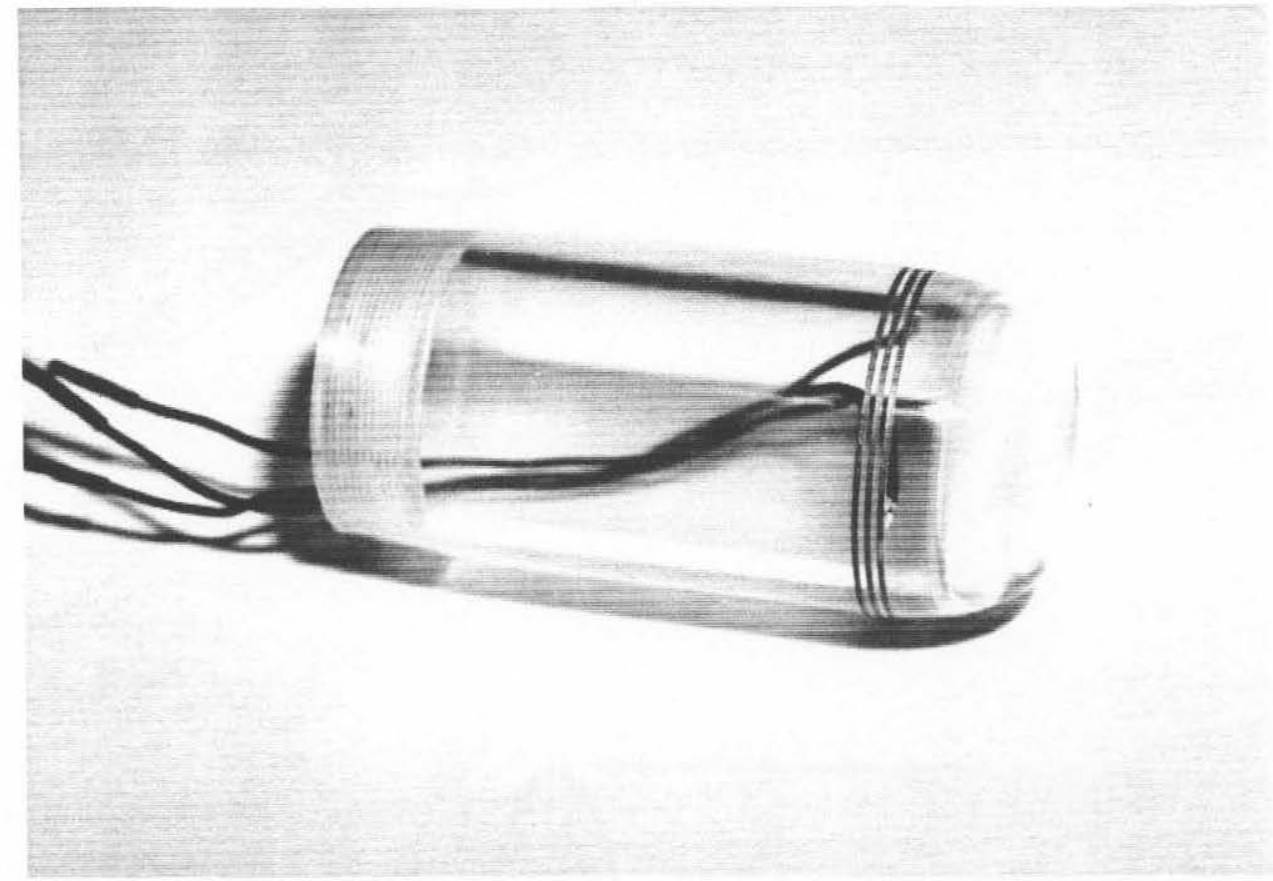

Figure 3.9 I.T.T.C. headform with circular electrodes. 


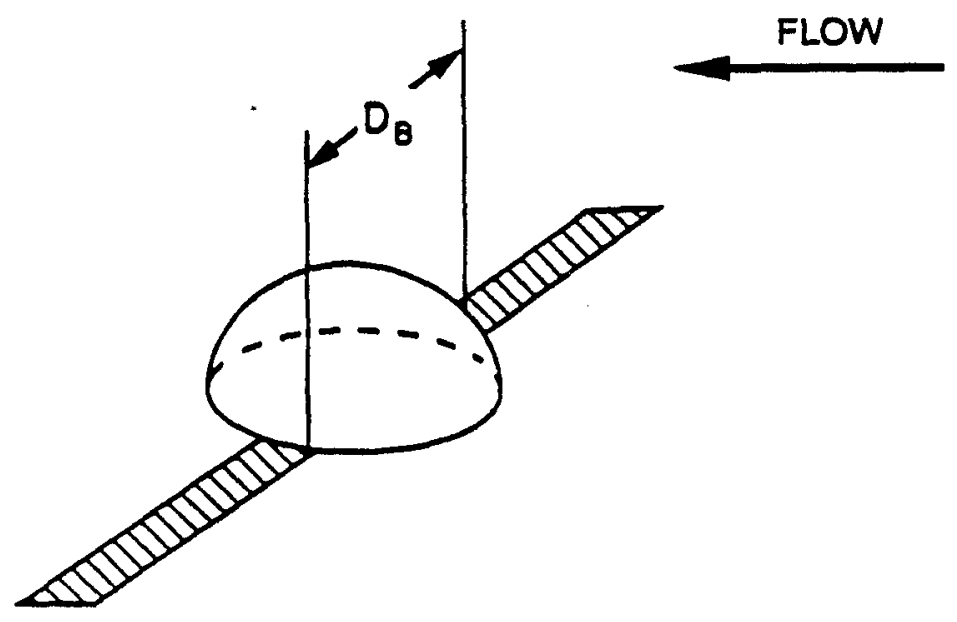

(a)

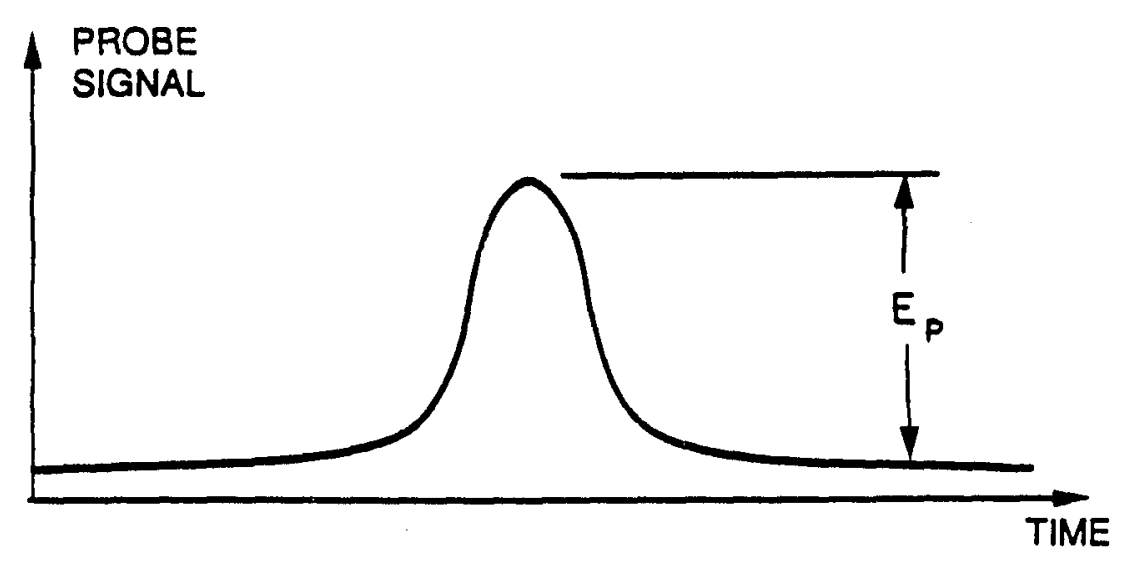

(b)

Figure 3.10 Illustration of base diameter measurement. As the bubble passes over the electrode, a percentage of the electrode circumference is covered (a), and this is proportional to the electrode signal (b). The maximum of the probe signal, $E_{P}$ is proportional to the maximum base diameter; $D_{B}$ 


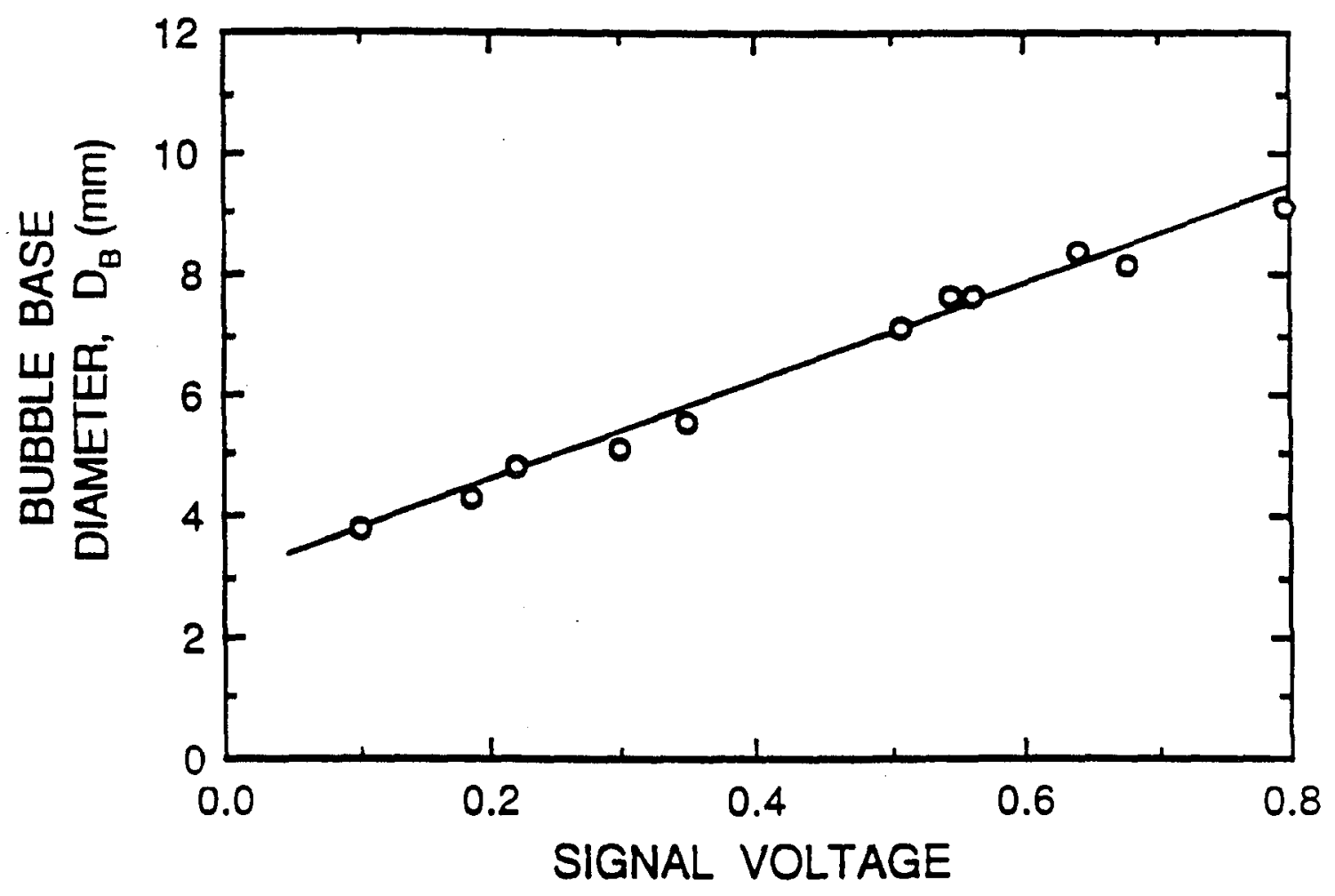

Figure 3.11 Example calibration curve for circular electrode system. Curve is for I.T.T.C. body. 

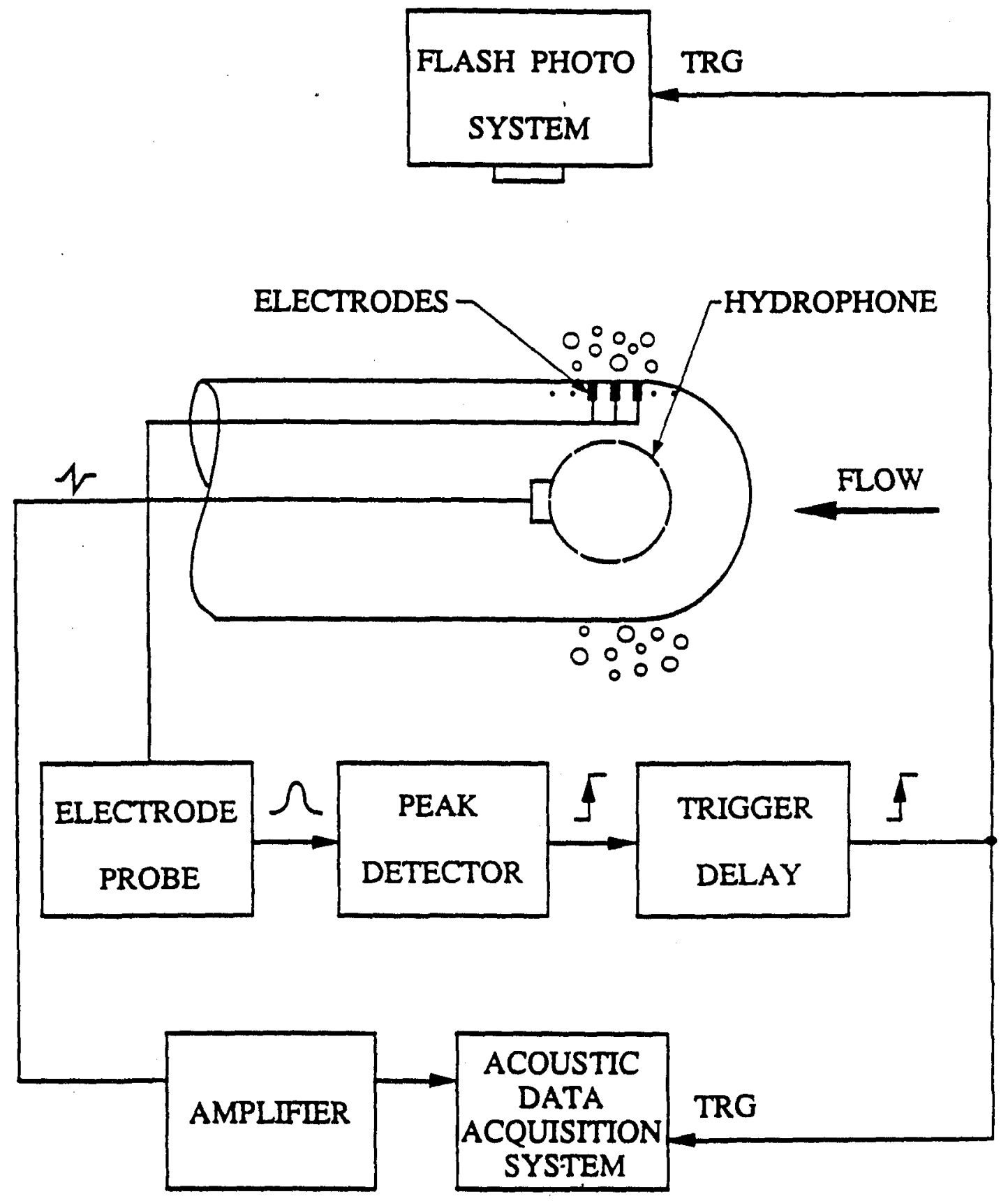

Figure 3.12 Schematic diagram of patch electrode experiment. 


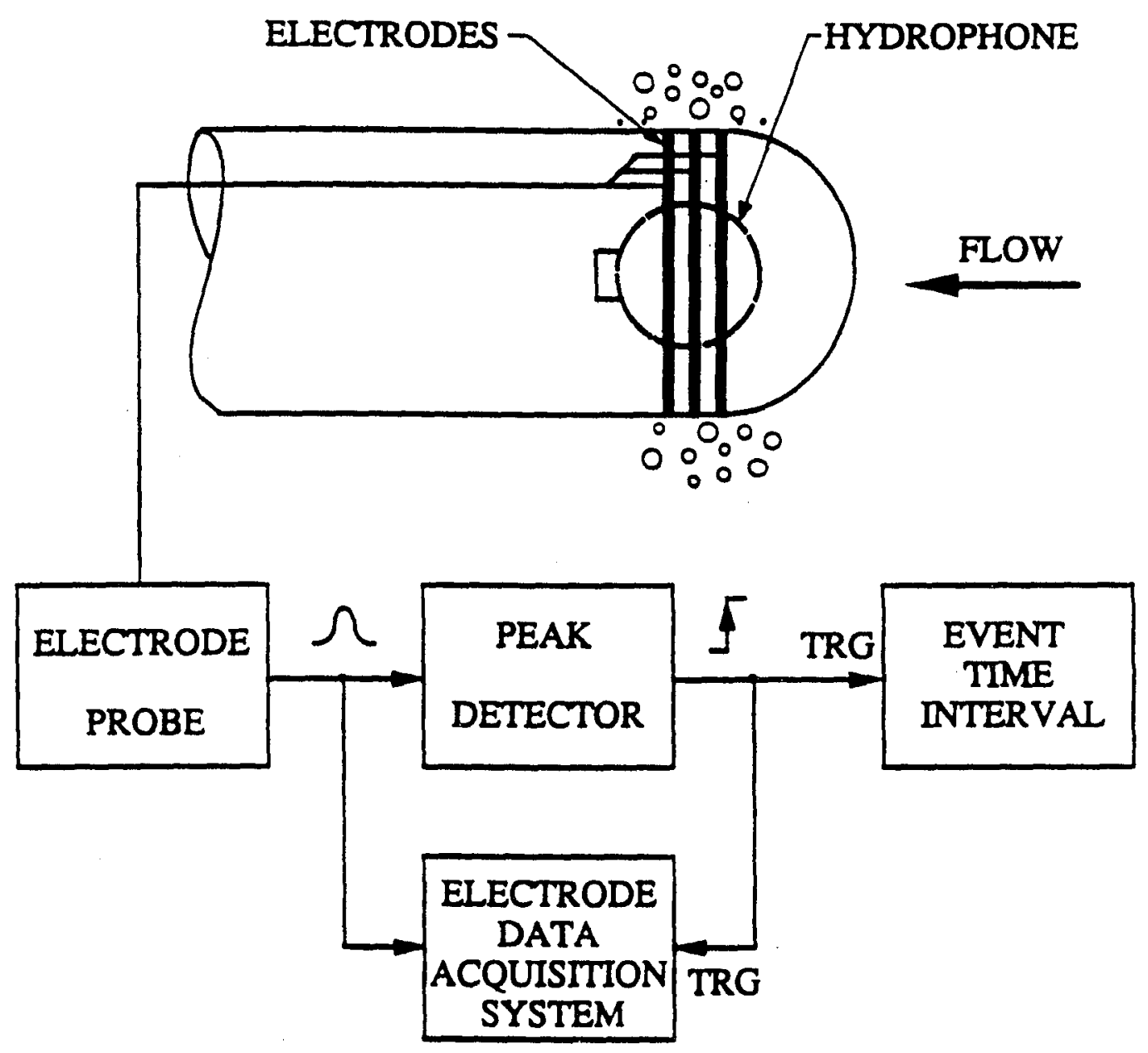

Figure 3.13 Schematic diagram of bubble statistics experiment for circular electrodes. 


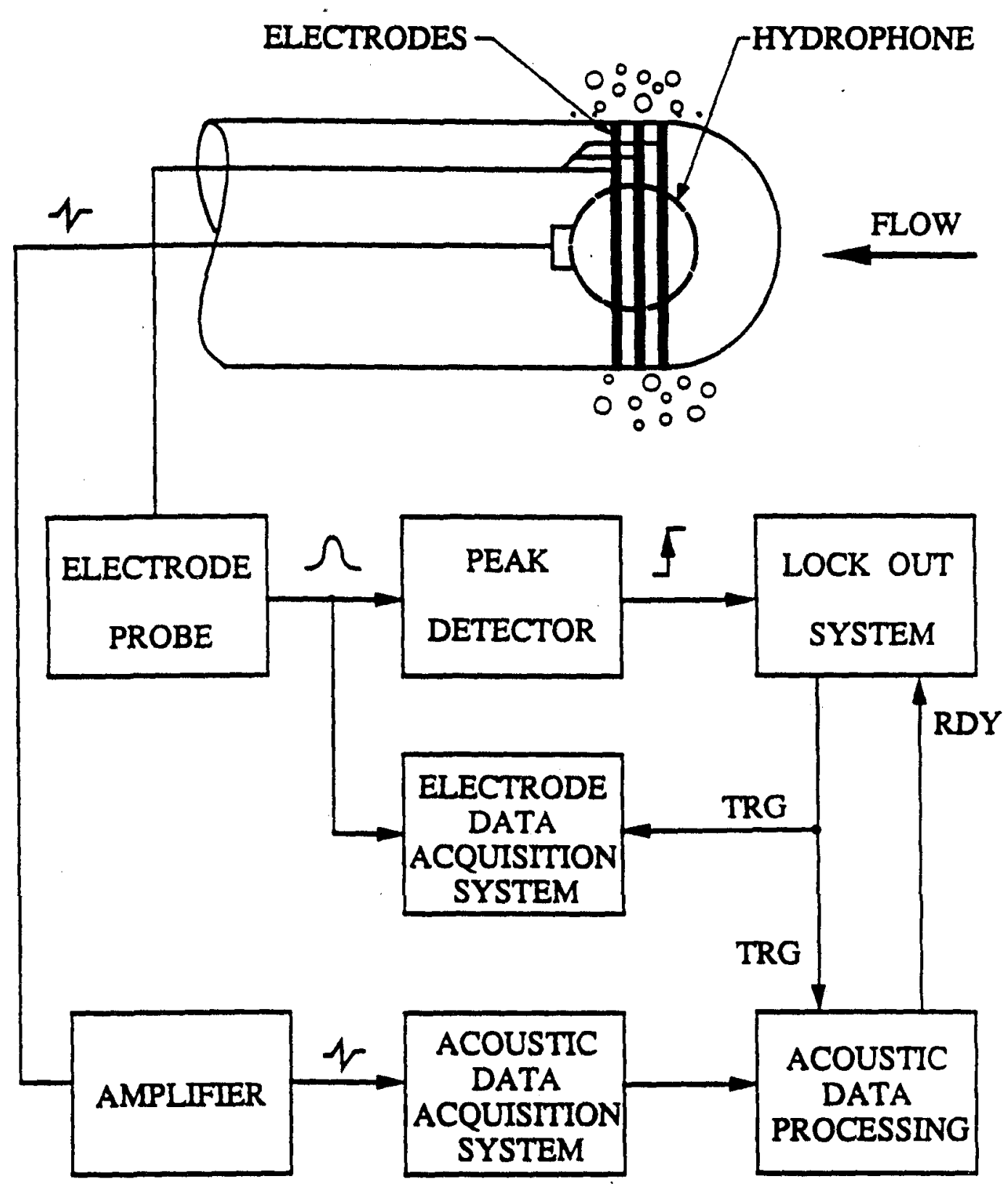

Figure 3.14 Schematic diagram of bubble acoustics experiment for circular electrodes. 


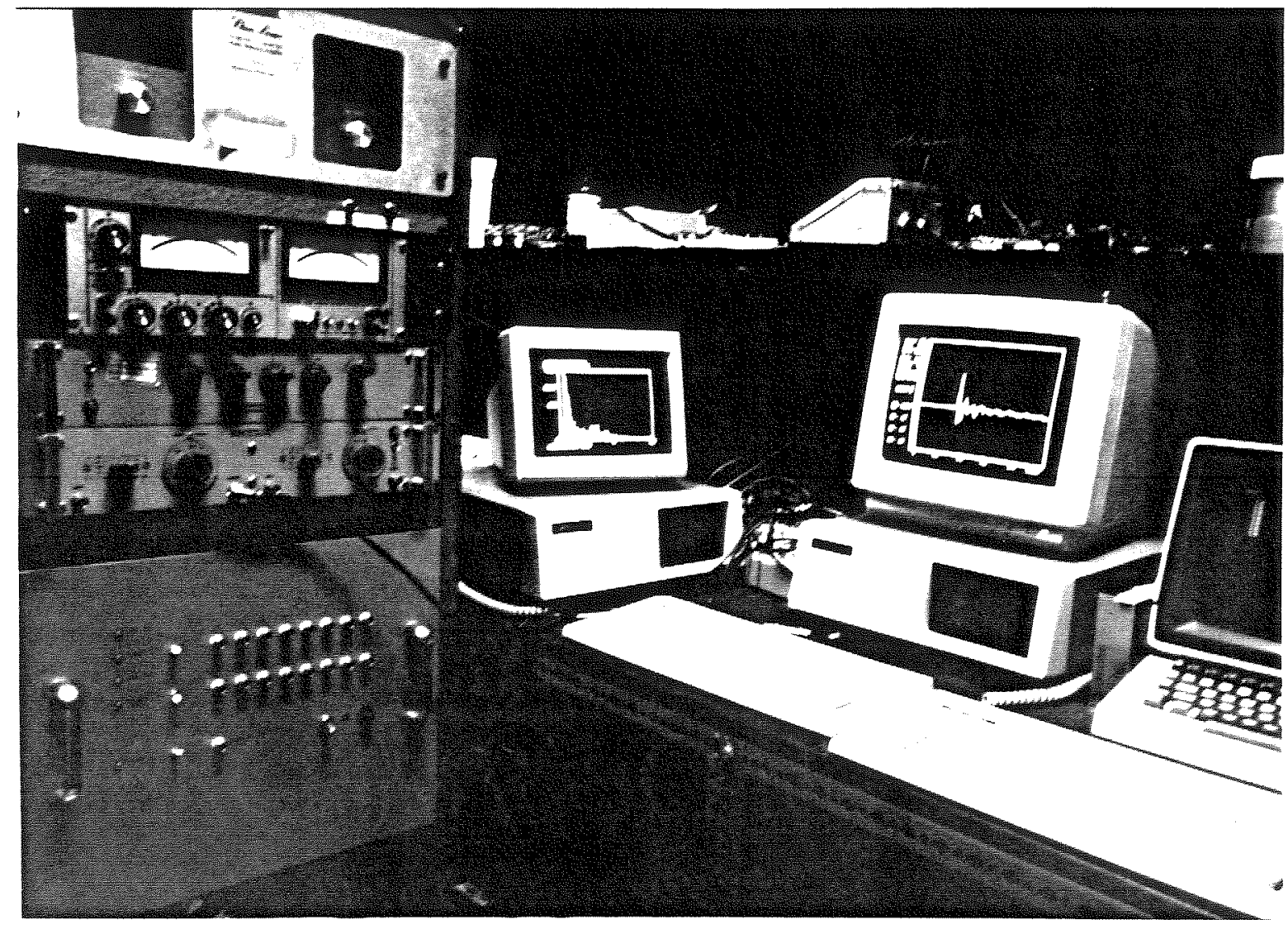

Figure 3.15 Photograph of electronic equipment. 


\section{CHAPTER 4}

\section{Observations of Single Cavitation Bubbles}

\subsection{Introduction}

Patch electrodes on both the Schiebe and I.T.T.C. headforms were used to obtain a series of photographs of naturally occurring cavitation bubbles at different stages in their trajectory. These photographs may be compared with those obtained by previous researchers. The most famous examples are those of Knapp and Hollander (1948) and Ellis (1952), whose observations of bubbles on axisymmetric headforms formed the basis of many cavitation studies. Recently, Hamilton (1981) was able to photograph bubbles that occurred on the surface of a Schiebe body.

Although significant information has been gained from the above studies, the photographic image quality of these experiments has not been entirely satisfactory. Bubbles have only been observed in profile or plan view, and the various lighting schemes have produced images only of the bubble outline. Furthermore, many photographs must be taken to capture a bubble randomly occurring in the proper position, thus limiting the number of photo sets.

By using the patch electrode probe, these problems were overcome. Bubbles passing over a specific patch will produce a signal, and cameras that had previously been focused on the electrode may then be triggered to photograph the bubble. In this way, close-up plan and profile views of the bubble were recorded, and this process could be easily repeated to produce any number of photo sets.

\subsection{General Observations}

Cavitation bubbles were generated on both headforms over a range of cavitation numbers. The cavitation number was varied between the traveling bubble cavitation inception value, $\sigma_{i}$, and the value at which attached cavitation occurred, $\sigma_{a c}$. The inception index on both bodies was strongly dependent on the ambient nuclei number distribution. Inception occurred on the Schiebe body at cavitation 
numbers as high as $\sigma_{i}=0.65$, and on the I.T.T.C. body at $\sigma_{i}=0.58$ for tunnel water of 6 to $7 p p m$ air content. However, on both bodies the inception index was reduced to about $\sigma_{i}=0.50$ immediately after deaeration. Any definition of the bubble cavitation inception index must therefore be associated with a particular free stream nuclei number distribution. The attached cavitation formation index for the Schiebe body was $\sigma_{a c}=0.40$ and for the I.T.T.C. body $\sigma_{a c}=0.41$. These values were almost constant over the fairly narrow range of Reynolds numbers of the experiments $\left(R e=4.4 \times 10^{5}-4.8 \times 10^{5}\right)$.

Before detailing the results from each headform, an observation can be made for both geometries. For a given tunnel velocity and cavitation number, the maximum volume of the bubble was quite uniform. Although the incoming nuclei diameter ranged over almost three orders of magnitude, the maximum cavitation bubble volume varied over only one order of magnitude. This phenomenon will be addressed below.

For both headforms, the growth phase of the nuclei was very similar to that described in the original observations of Knapp and Hollander (1948) and Ellis (1952). Initially, the bubbles would almost uniformly take on a hemispherical or "cap" shape over most of their trajectory and move extremely close to the headform surface; only very occasionally would quasi-spherical bubbles be observed at a distance above the surface. The bubbles were observed to ride over the boundary layer, which for both bodies is approximately $400 / \mathrm{mum}$ thick in the region of bubble growth (Gates (1977)). Small waves could be observed on the bubble surface in many instances. As the bubbles reach their maximum volume they become somewhat elongated in the direction normal to their motion while their thickness normal to the surface remains relatively constant. At this point, the difference in the flows around the two bodies begins to cause differences in the bubble dynamics. 


\subsection{Schiebe Body Results}

The Schiebe body was designed to suppress laminar separation in the region of cavitation (Schiebe (1972)). It possesses a sharp pressure drop with a minimum pressure coefficient of -0.75 (Figure 4.1 ). Figure 4.2 represents a schematic drawing of the typical bubble evolution, and Figure 4.3 consists of a series of bubbles photographed at various stages during this process. After the bubble has reached its maximum volume, it begins to lose its cap-like shape and becomes elongated, progressing into a pyramid-like shape; the bubble thickness normal to the headform surface consistently decreases after reaching its maximum.

The bubble then collapses rapidly and develops an elongated shape. The elongation of the bubble and the formation of tubes is probably due to rotation of the bubbles caused by the shear in the boundary layer and nearby flow. As the bubble collapses it may fission into two or three tubes of collapsing vapor, and the residual gas in these tubes may cause a rebound to produce a rough bubble or group of bubbles after collapse.

\subsection{I.T.T.C. Body Results}

The I.T.T.C. headform has a relatively smooth pressure drop with a minimum pressure coefficient of -0.62 . A distinguishing feature of this headform is that, unlike the Schiebe body, it possesses a laminar separation region (Figure 4.1). Figure 4.4 represents a schematic drawing of the typical bubble evolution, and Figure 4.5 presents a series of bubbles photographed at various stages of this development. The bubble has a cap-like shape until it reaches its maximum volume where it then becomes further elongated moving into the wedge-like shape. However, unlike the bubbles on the Schiebe body, the cavity starts to lift off the surface and begins to roll up into a snout-like shape. This may be due to recirculating flow associated with the separation region or the stretching of the bubble in the velocity gradient. As it collapses, the "snout" continues to roll up into a vapor tube, eventually collapsing 
to produce a rough bubble.

On both the Schiebe and I.T.T.C. headforms, the rough bubble or group of bubbles that is formed after collapse is sheared by the surface flow and usually disperses into smaller bubbles on the order of $50 \mu \mathrm{m}$, although a second collapse and rebound is not uncommon. The mean lifetime of a bubble depends upon the tunnel velocity, cavitation number, and initial nuclei size, but, for most of the observed bubbles on both headforms, it is approximately $3 m s$.

The laminar separation on the I.T.T.C. body has been carefully studied in the context of its effect on attached cavitation (Arakeri and Acosta (1973)). Clearly, the separated flow also influences bubble cavitation. Cavitation bubbles were observed riding over the separation bubblem, and, as seen in Figures 4.4 and 4.5 , the underside of the bubbles became roughened as they passed over the region of turbulent reattachment. These local flow disturbances seem to be shearing vapor off the underside of the bubble, leaving a trail of much smaller bubbles. This phenomenon was not observed for bubbles on the Schiebe body.

Furthermore, some bubbles were seen to cause local attached cavitation. When the operating cavitation number was close to the attached cavity formation index, trailing "streamers" were often observed downstream of the cavitation bubble (Figure 4.6). These streamers were generally associated with the larger bubbles on the I.T.T.C. body (and occasionally on the Schiebe body) and were seen to develop gradually at the location of the laminar separation point (Arakeri and Acosta (1973)). As the bubble is swept downstream, the streamers continue to grow, and in may cases persist even after the bubble has collapsed. Why these bubbles cause the attached cavitation streamers at the lateral extremities of the bubble is unclear. This phenomena has also been observed with travelling bubble cavitation on hydrofoils (i.e., van der Meulen (1980)), and explanations for it have ranged from a wake flow to a horseshoe vortex (Rood (1989)). The process could be considered an inception mechanism for attached cavities. 


\subsection{Conclusions}

The bubble growth and collapse mechanisms described above are the result of many detailed observations, and the effects of the surface flow are quite evident. Photographs presented by Hamilton (1981) are consistent with the observations of this study, although the relatively limited image quality of these photographs makes detailed interpretation difficult.

The classic observations of Knapp and Hollander (1949) (Figures 4.7 and 4.8) may be compared those of this study. Both experiments revealed that bubbles travelling near surfaces are cap shaped, and the gross characteristics of growth and collapse are similar. However, the pressure distribution on the ogive of Knapp and Hollander provided for a long and steady growth, and the bubbles often retained a quasi-spherical shape even near the final stages of collapse. As Figure 4.8 indicates, these bubbles would often rebound many times, maintaining their quasi-spherical shape after each collapse. The bubbles observed in this study usually rebounded only once and lost most of their coherent shape after the first collapse.

This difference may be explained by noting that the water tunnel facility used by Knapp and Hollander was not equipped with any deaeration system, and extremely bubbly flows were used to increase the odds of photographing a cavitation event. Consequently, the cavitating nuclei observed by Knapp and Hollander were generally large, containing more undissolved gas. Increasing the amount of residual gas reduces the violence of the bubble collapse, making coherent rebounds possible. On the other hand, the nuclei populations of the present study were quite small, and the cavitation bubbles observed were almost entirely vaporous. These bubbles will collapse violently, and coherent rebounds are less likely. The effects of undissolved gas on cavitation bubble dynamics will be addressed in more detail in Chapter 5 .

Photographs of bubbles presented by Ellis (1952) show many of the same features as in this study. Principally, bubbles formed close to the headform also 
progressed from a cap shape to a wedge shape before collapse, although the collapse mechanism is difficult to distinguish in the silhouette images. Ellis observed that the bubble surface profile approximately coincided with lines of constant pressure, which may account for the wedge shape of the bubble. Figure 4.9 shows the isobaric lines computed for flow around the Schiebe body (Schiebe (1972)), and the dashed line represents the observed outline of an average bubble. Clearly, the bubble is being shaped by the pressure gradients close to the surface.

Returning to the present study, collapse mechanisms for bubbles on both headforms were discerned through the study of many photographs. A composite picture is presented in Figure 4.10 for the Schiebe body, with example photos in Figure 4.11. For the I.T.T.C. body, similar results are given in Figures 4.12 and 4.13. Previous researchers have noted the generation of a liquid microjet in bubbles collapsing near a solid surface (Lauterborn and Bolle (1975) and Kimoto (1987), for example), and this microjet is suspected to be the main cause of cavitation erosion damage. Although many photographs were taken, a reentrant microjet was not observed in any of the photographs of bubble collapse, although the jet may have occurred too rapidly to be detected.

Two factors may account for the possible absence of microjet formation. First, the intensity of the bubble collapse may be correlated with the magnitude of the pressure difference across the bubble wall at the initial stages of collapse, and a violent collapse, with greater bubble wall velocities, may favor the formation of the microjet. Artificially produced cavitation bubbles near surfaces, which often produce microjets, have static pressure differences across the bubble wall on the order of 80 to $300 \mathrm{kPa}$ (van der Meulen (1989)). The bubbles in this study, however, had static pressure differences on the order of $20 k P a$, possibly reducing the collapse intensity and inhibiting jet formation.

Furthermore, the collapse mechanisms described above reveal the lack of compact bubble geometries generally associated with jet formation. As a bubble 
collapses, fluid must replace the shrinking bubble volume, but the side of the bubble near a solid boundary may encounter a fluid deficit, causing the bubble centroid to move closer to the surface and inducing a jet to form. A collapse mechanism described above may not lead to this fluid deficit, reducing the possibility of jet formation. 

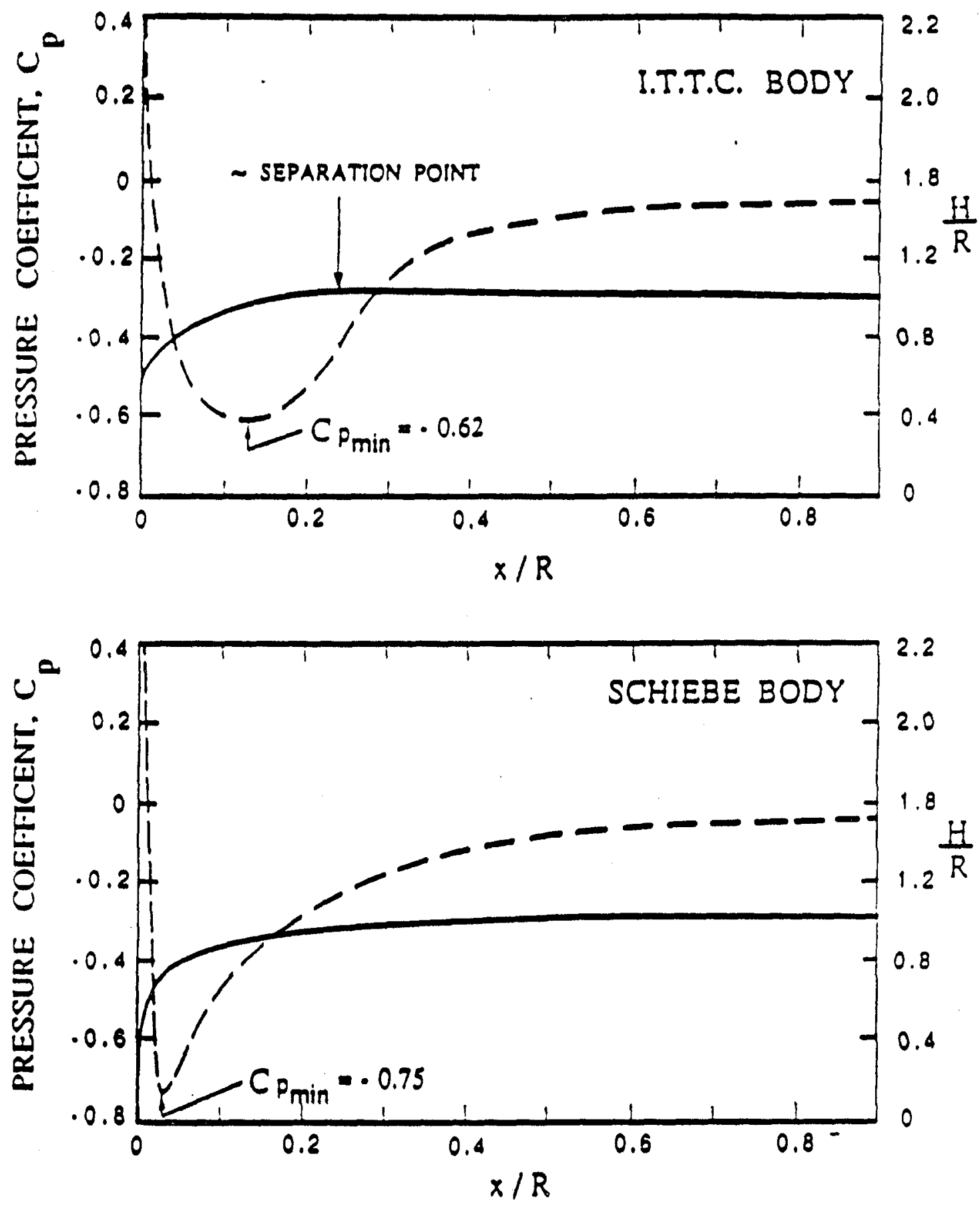

Figure 4.1 Pressure distributions on the I.T.T.C body and the Schiebe body. 


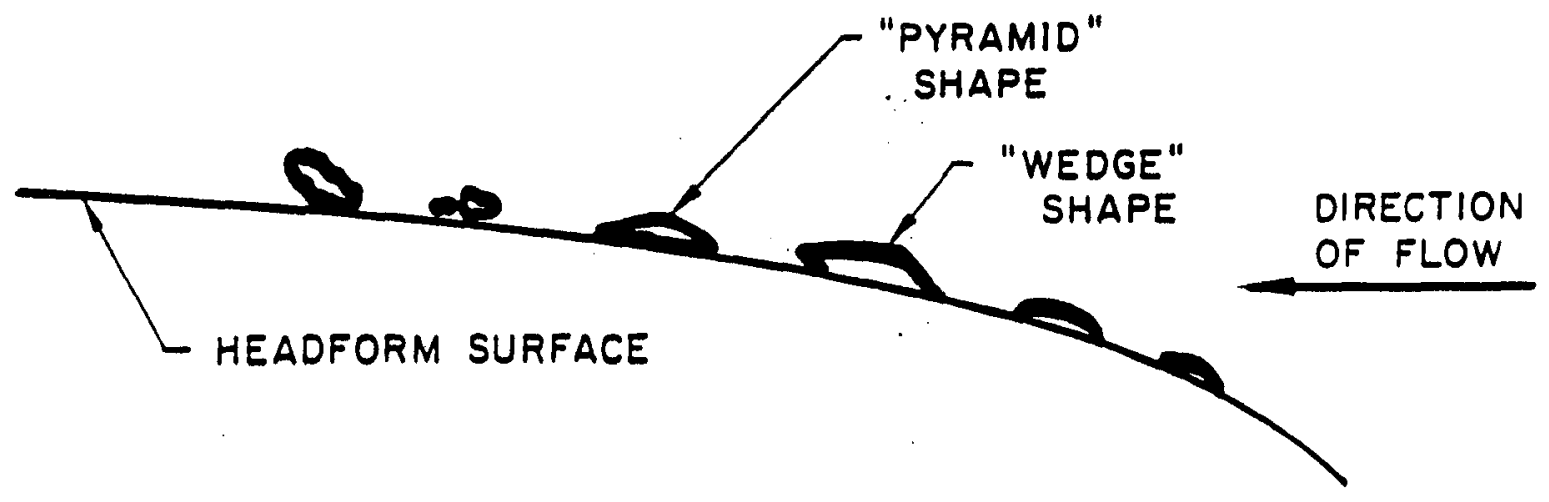

PLAN VIEW

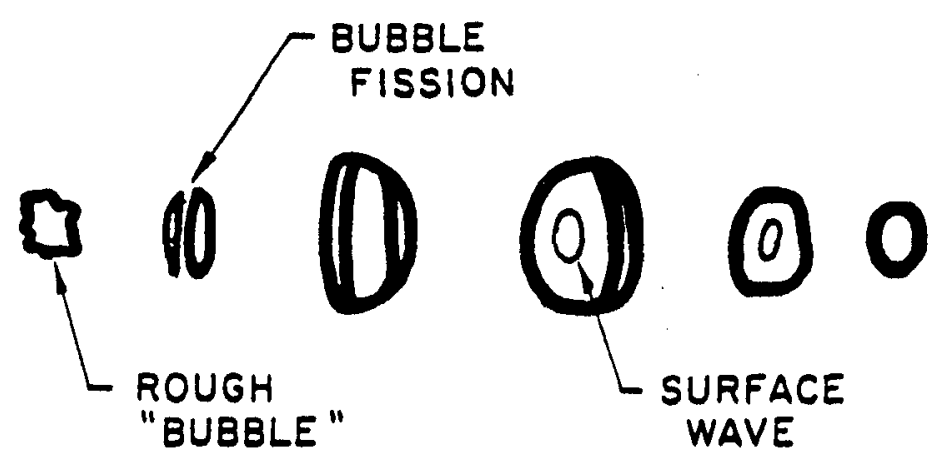

$-5 \mathrm{~mm}$

Figure 4.2 Schematic diagram of typical bubble trajectory on the Schiebe headform. 

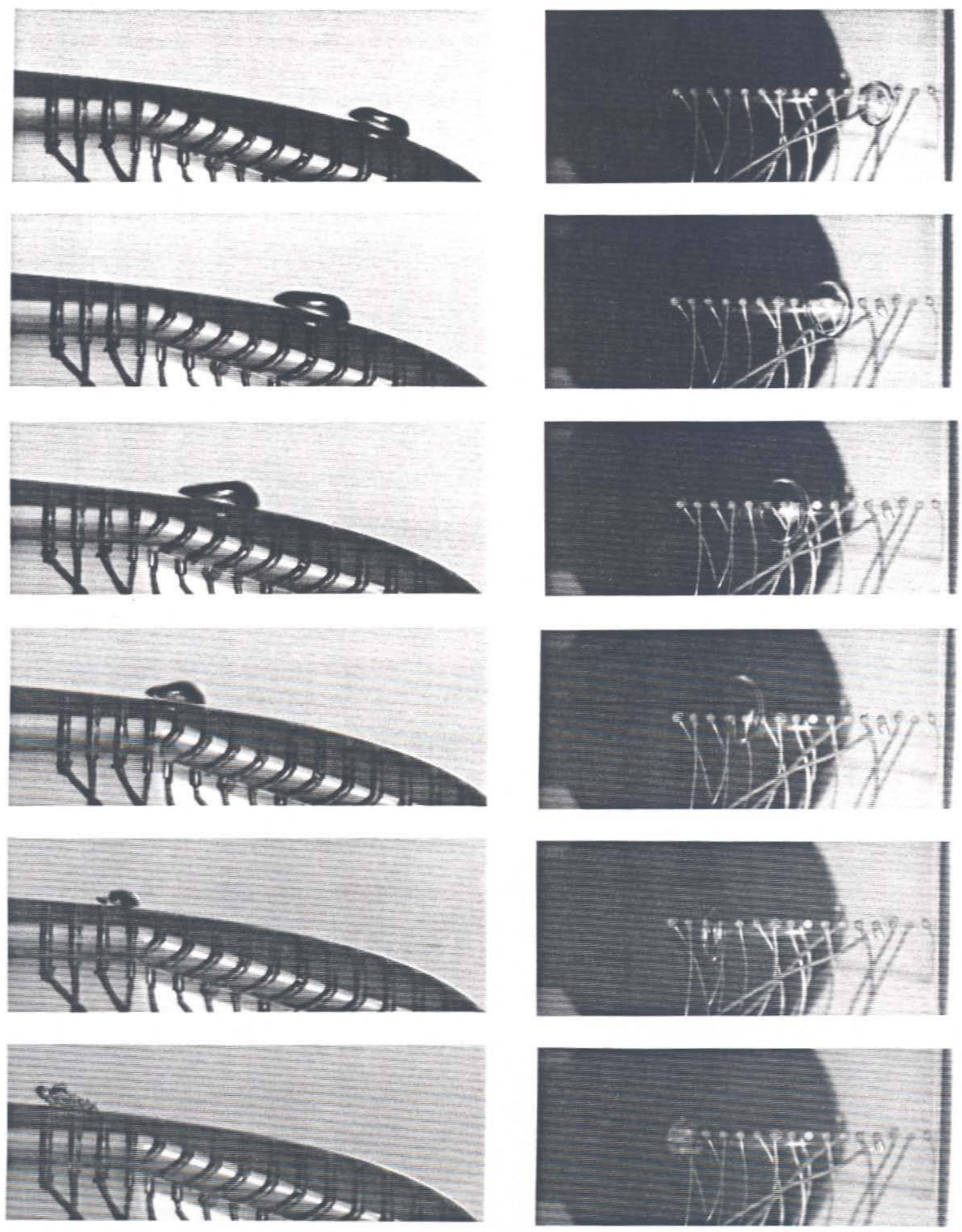

Figure 4.3 Series of photographs detailing typical bubble trajectory on the Schiebe headform, $U=9 \mathrm{~m} / \mathrm{s}$ and $\sigma=0.45$. 


\section{PROFILE VIEW}

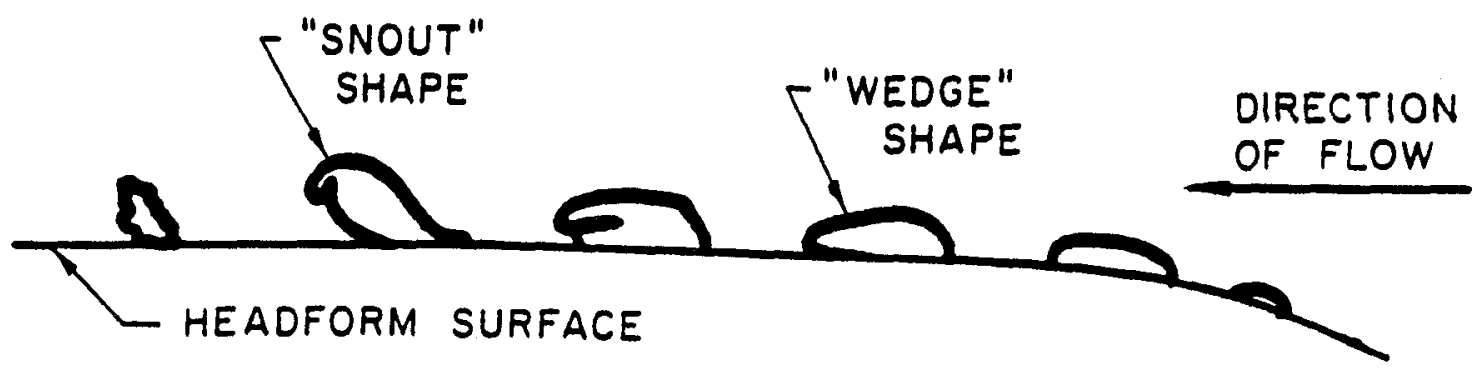

\section{PLAN VIEW}

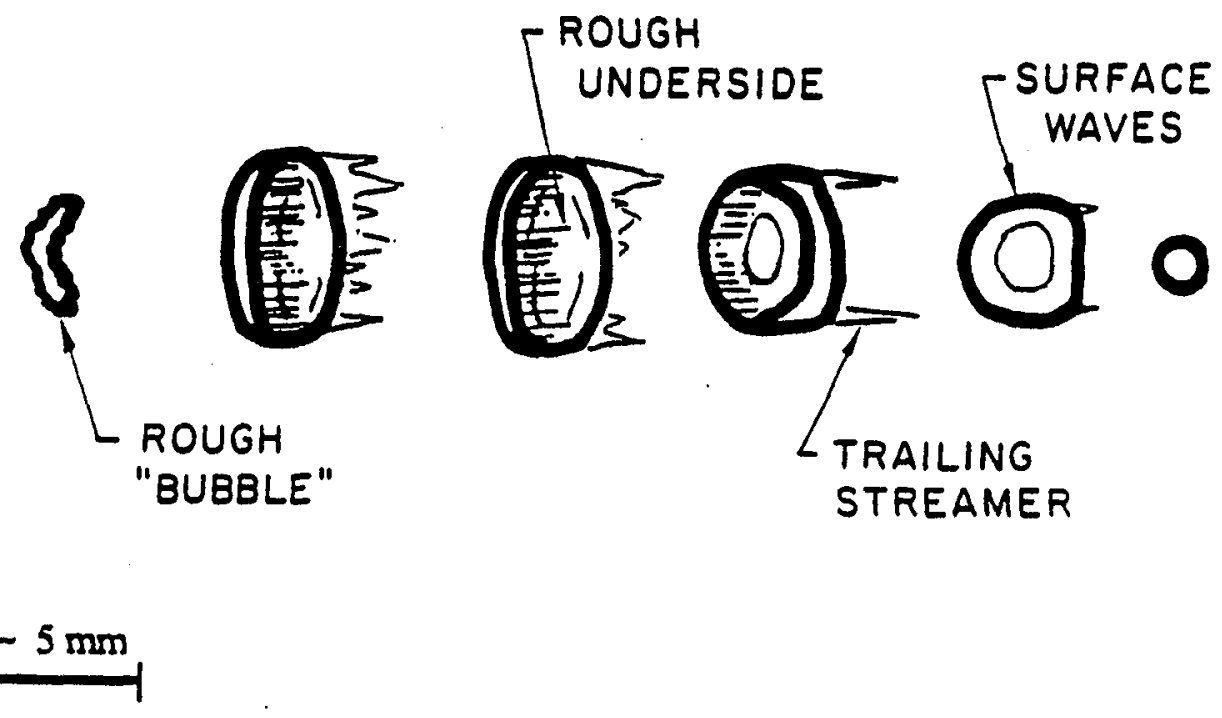

Figure 4.4 Schematic diagram of typical bubble trajectory on the I.T.T.C. headform. 

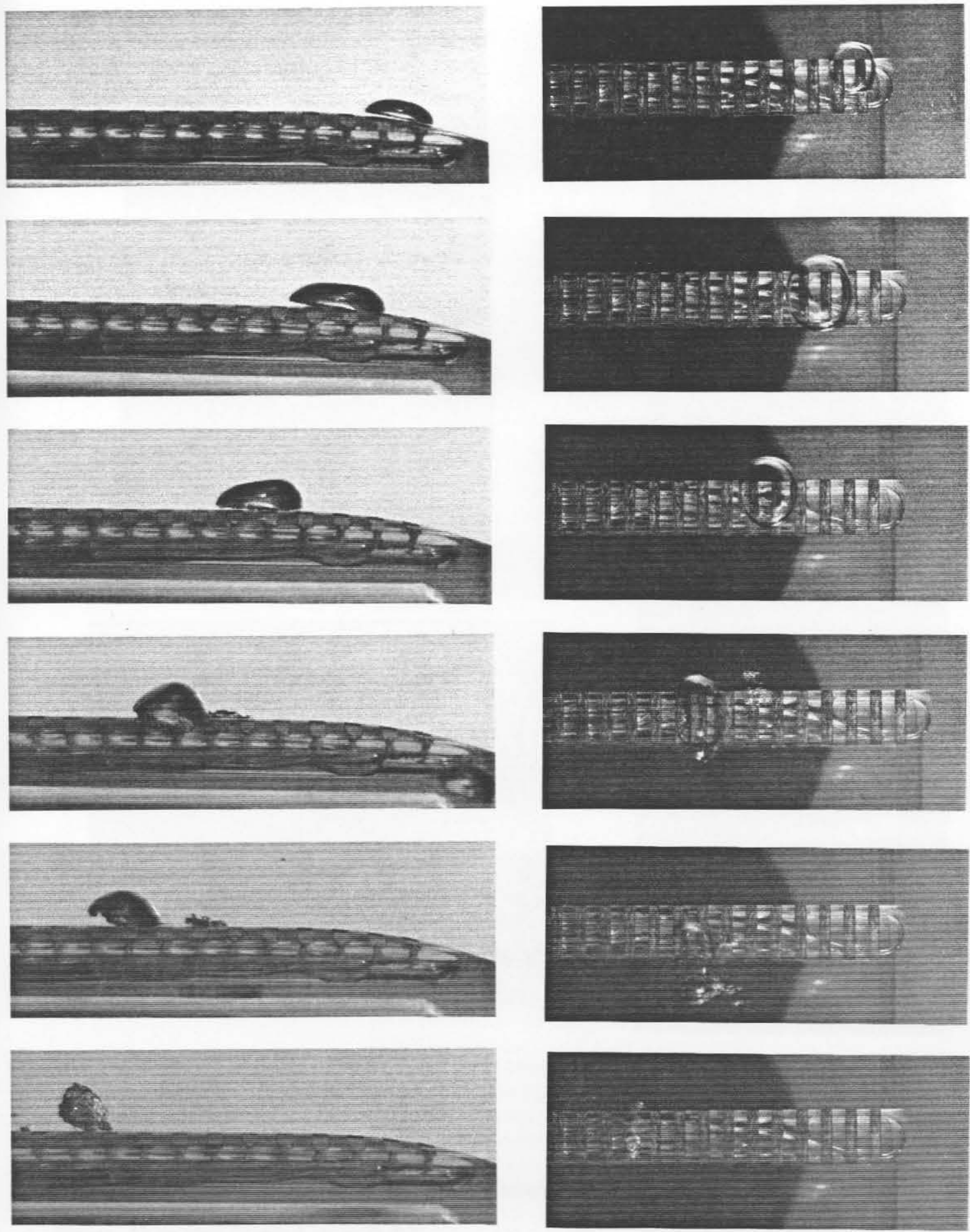

Figure 4.5 Series of photographs detailing typical bubble trajectory on the I.T.T.C. headform, $U=8.7 \mathrm{~m} / \mathrm{s}$ and $\sigma=0.45$. 

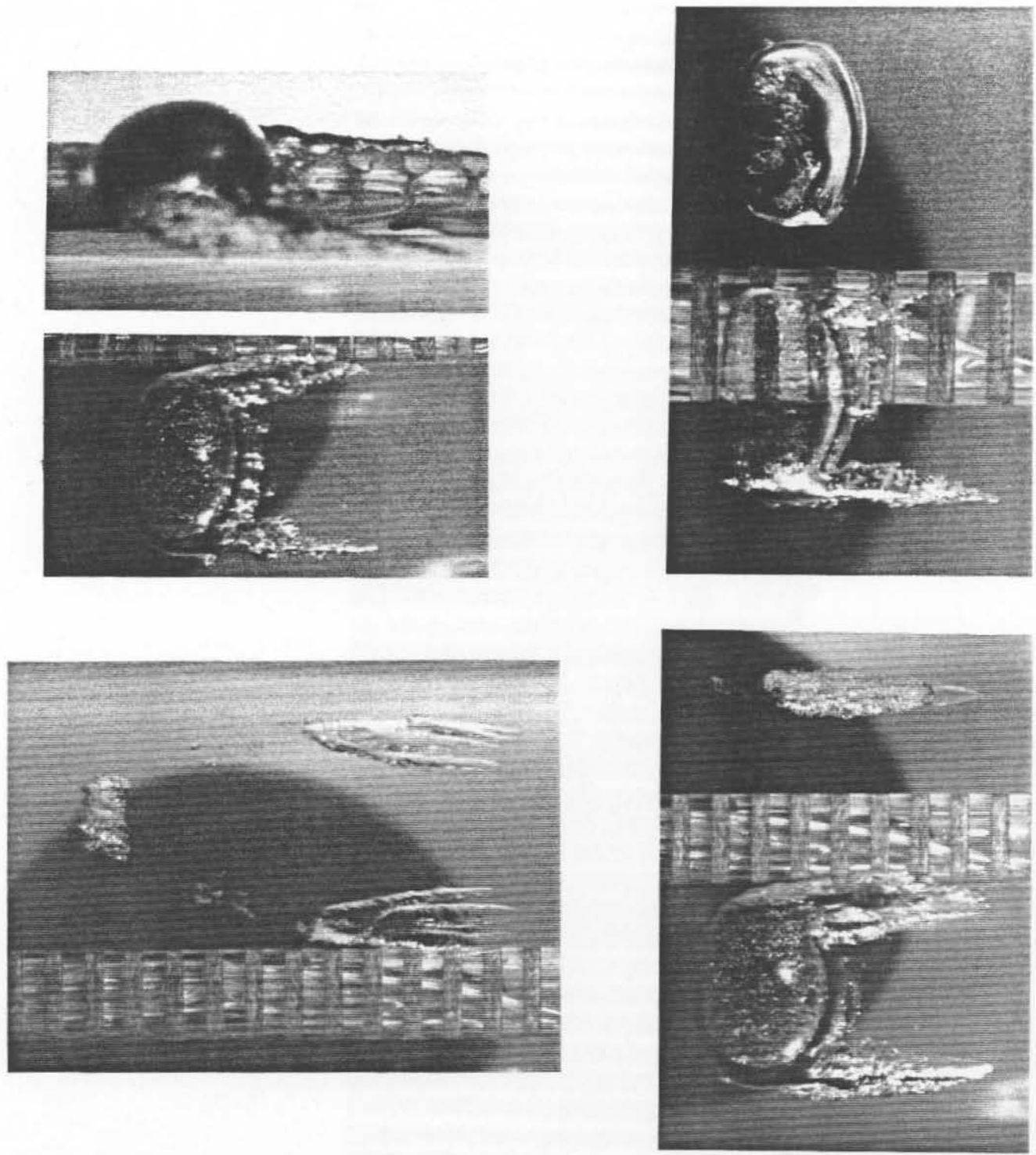

Figure 4.6 Series of photographs detailing bubbles with tails the I.T.T.C. headform, $U=9 \mathrm{~m} / \mathrm{s}$ and $\sigma=0.42$. 


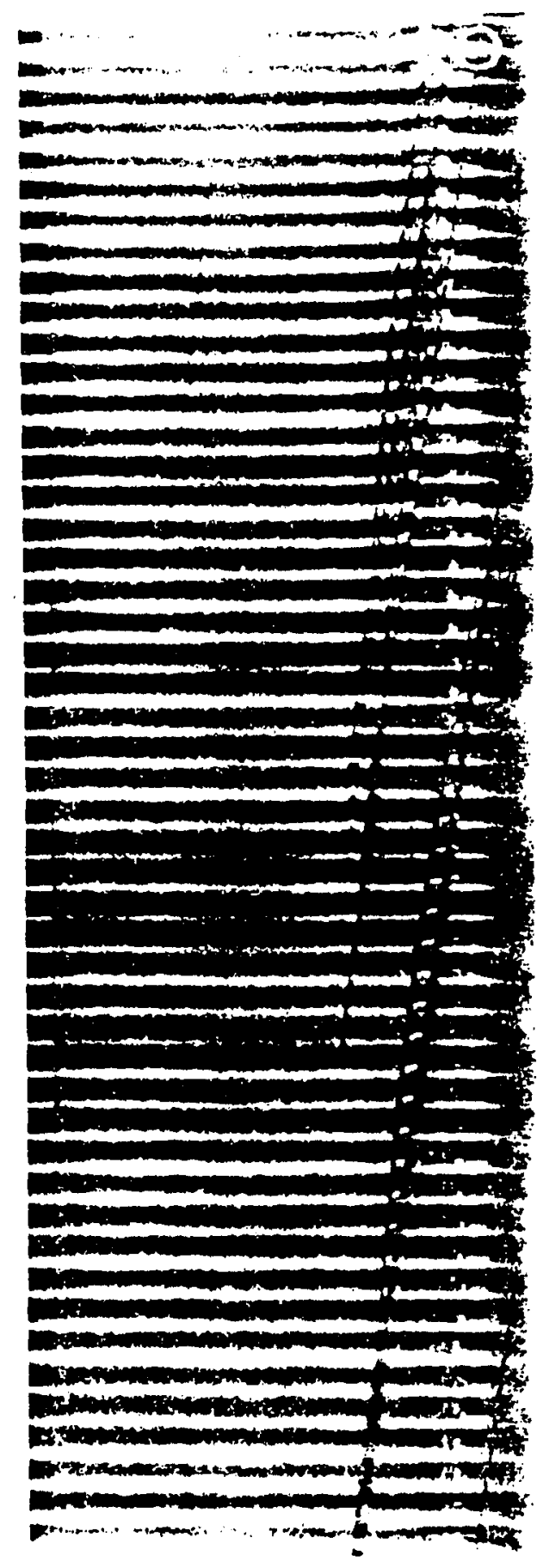

Figure 4.7 Photographs of bubbles taken by Knapp and Hollander. 


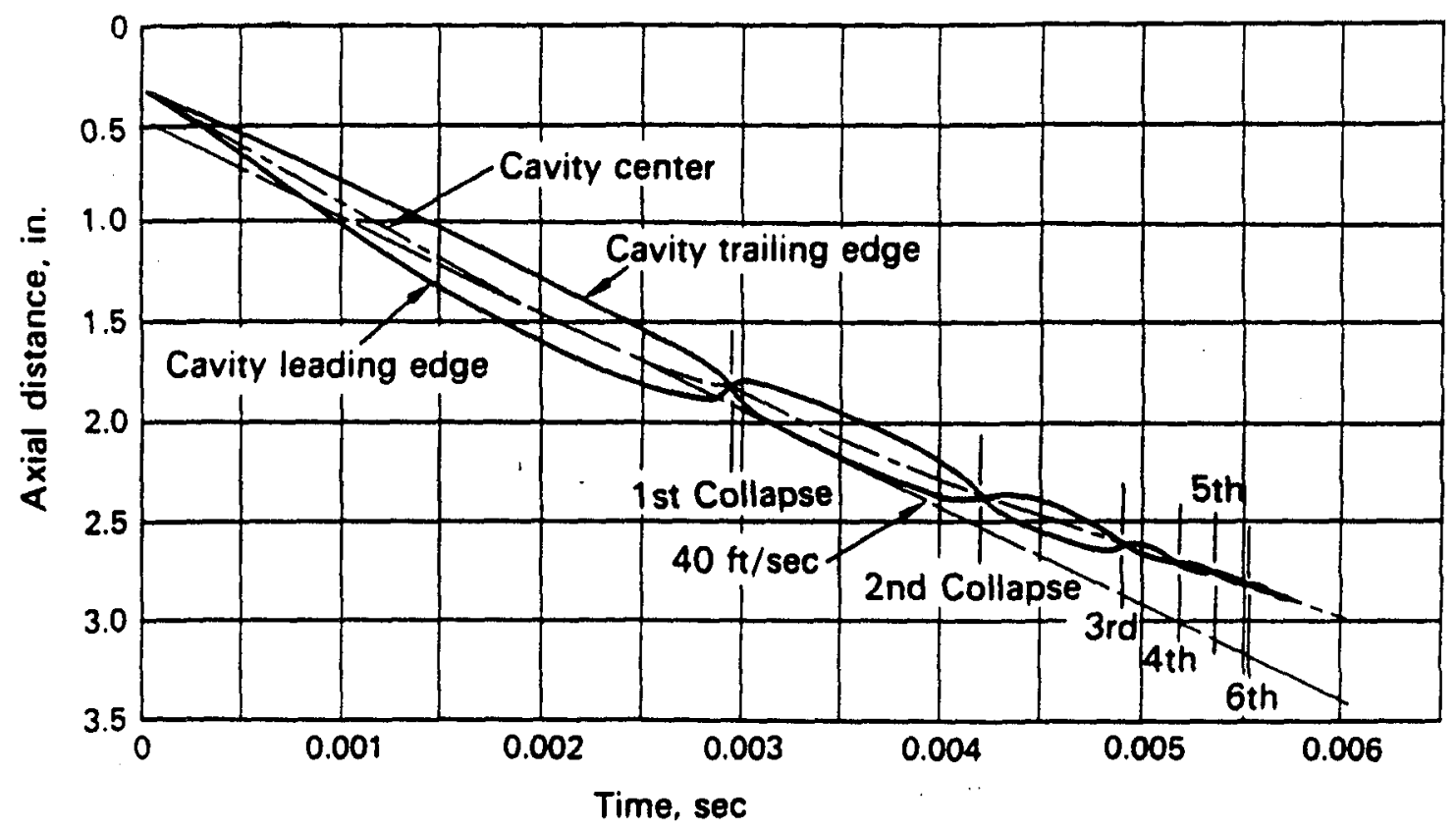

Figure 4.8 Typical bubble trajectory observed by Knapp and Hollander. 
$C_{P M}=-0.75$

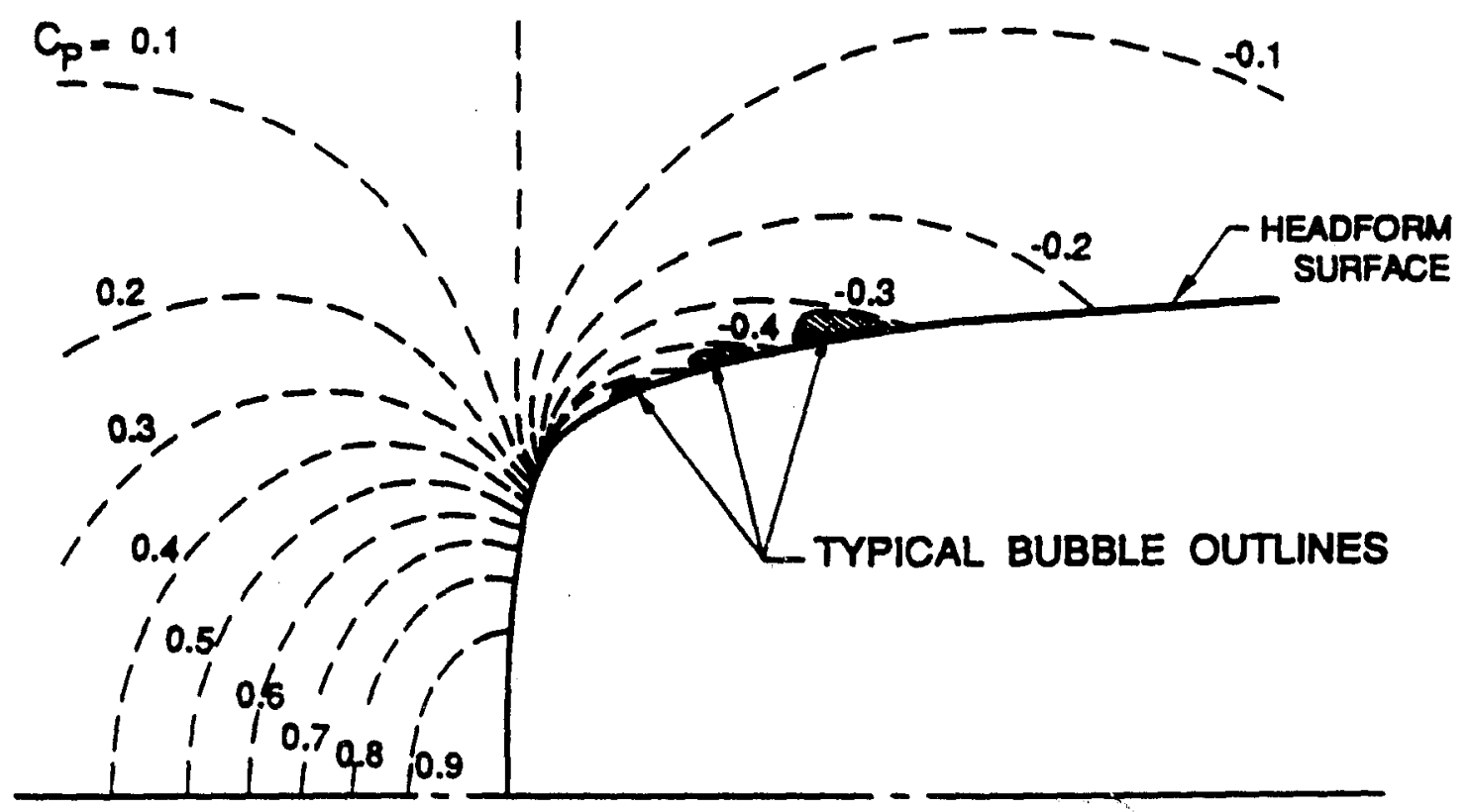

Figure 4.9 Schiebe body isobars and typical bubble profile. 


\section{PROFILE VIEW}

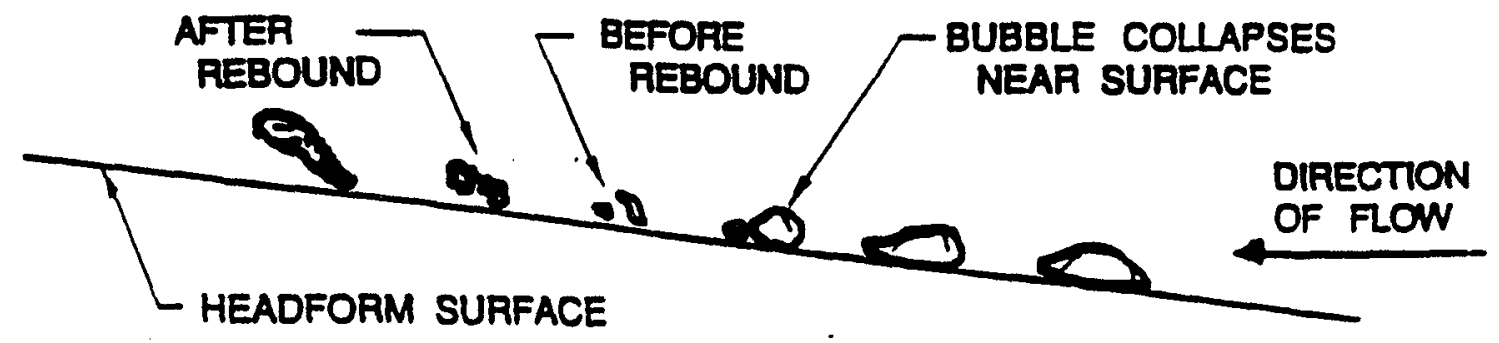

PLAN VIEW

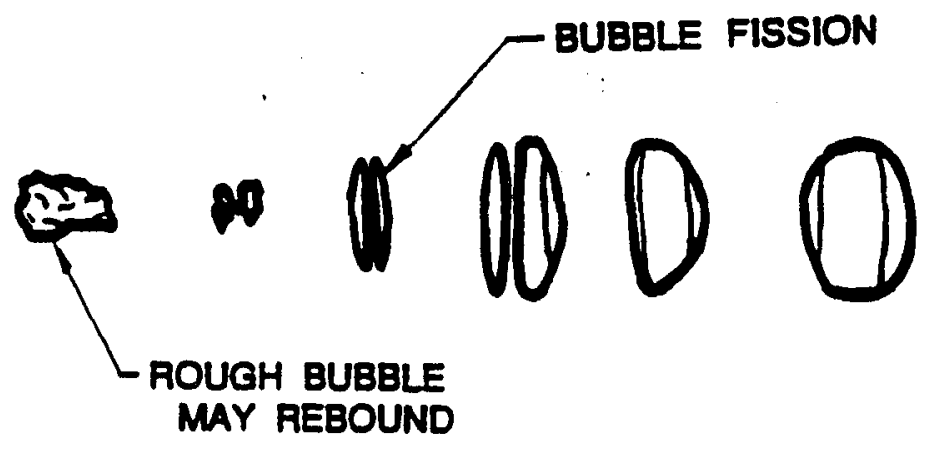

$-5 \mathrm{~mm}$

Figure 4.10 Schematic diagram of typical bubble collapse mechanism on the Schiebe headform. 

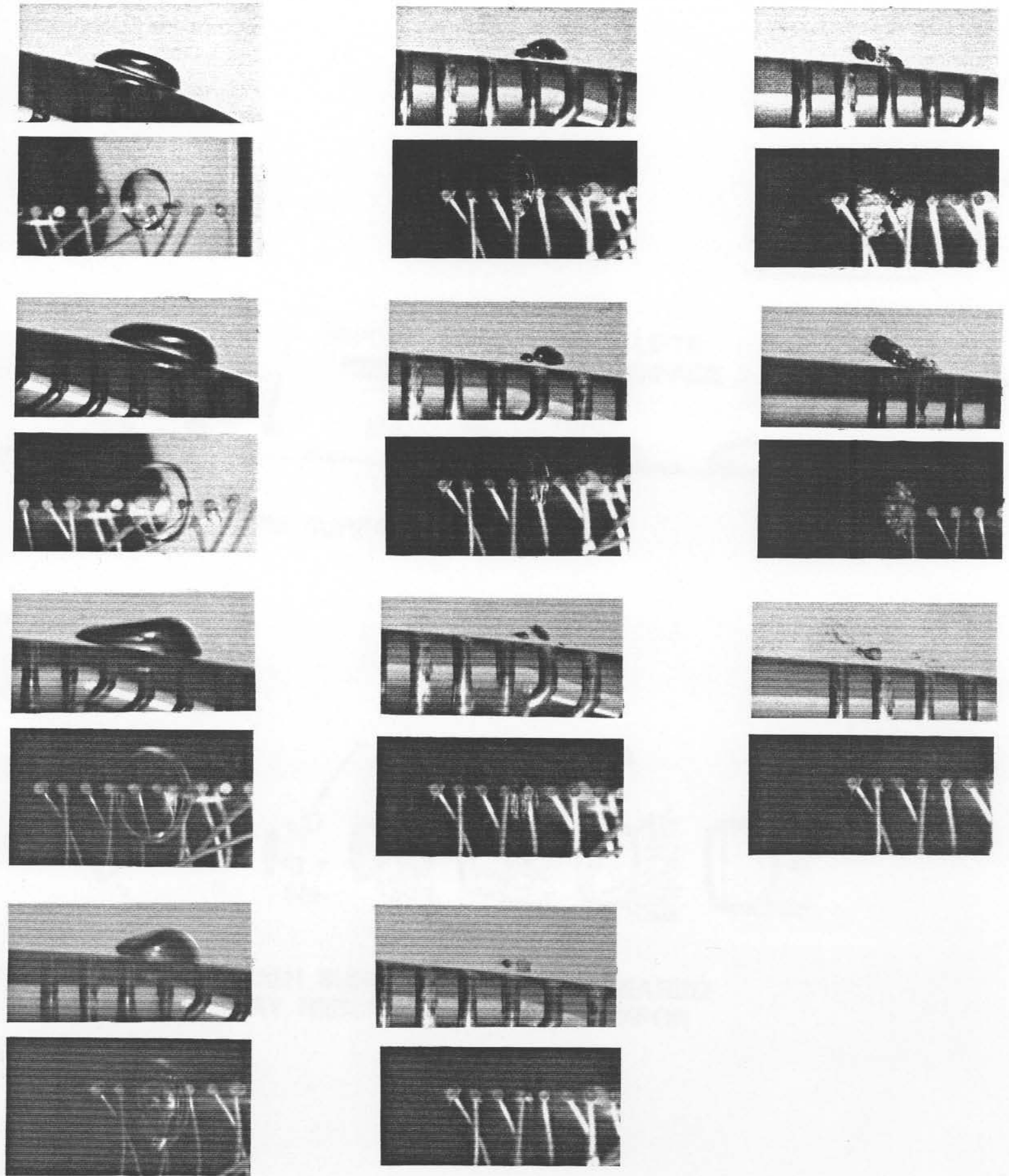

Figure 4.11 Series of photographs detailing typical bubble collapse mechanism on the Schiebe headform, $U=9 \mathrm{~m} / \mathrm{s}$ and $\sigma=0.45$. 
PROFILE VIEW

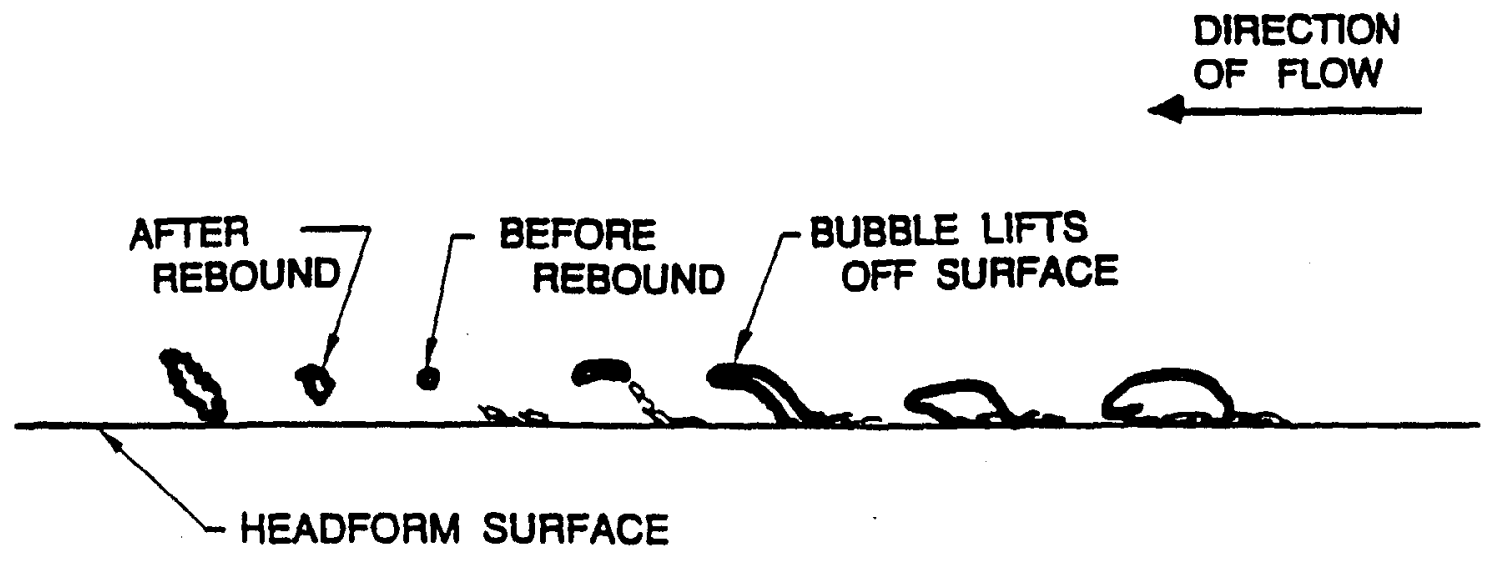

PLAN VIEW

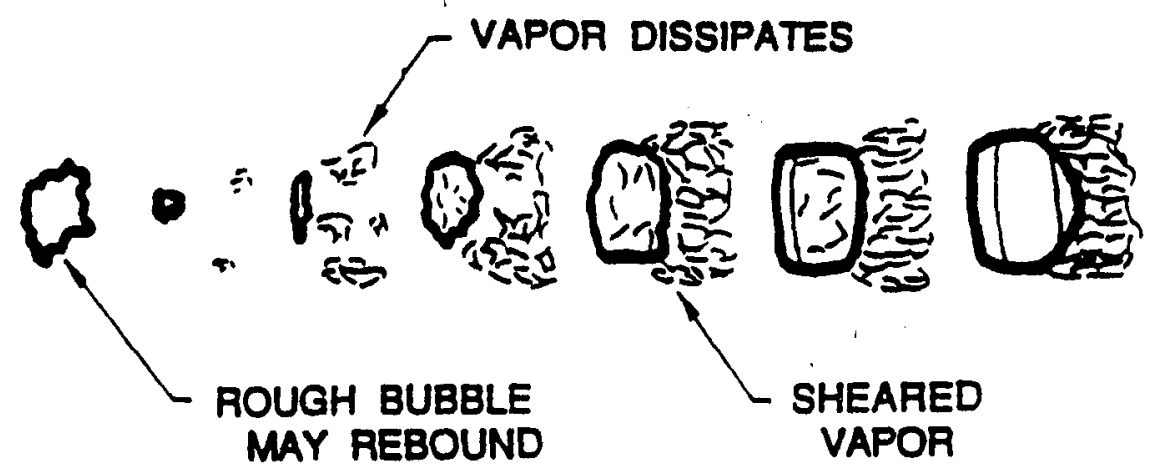

$-5 \mathrm{~mm}$

Figure 4.12 Schematic diagram of typical bubble collapse mechanism on the I.T.T.C. headform. 

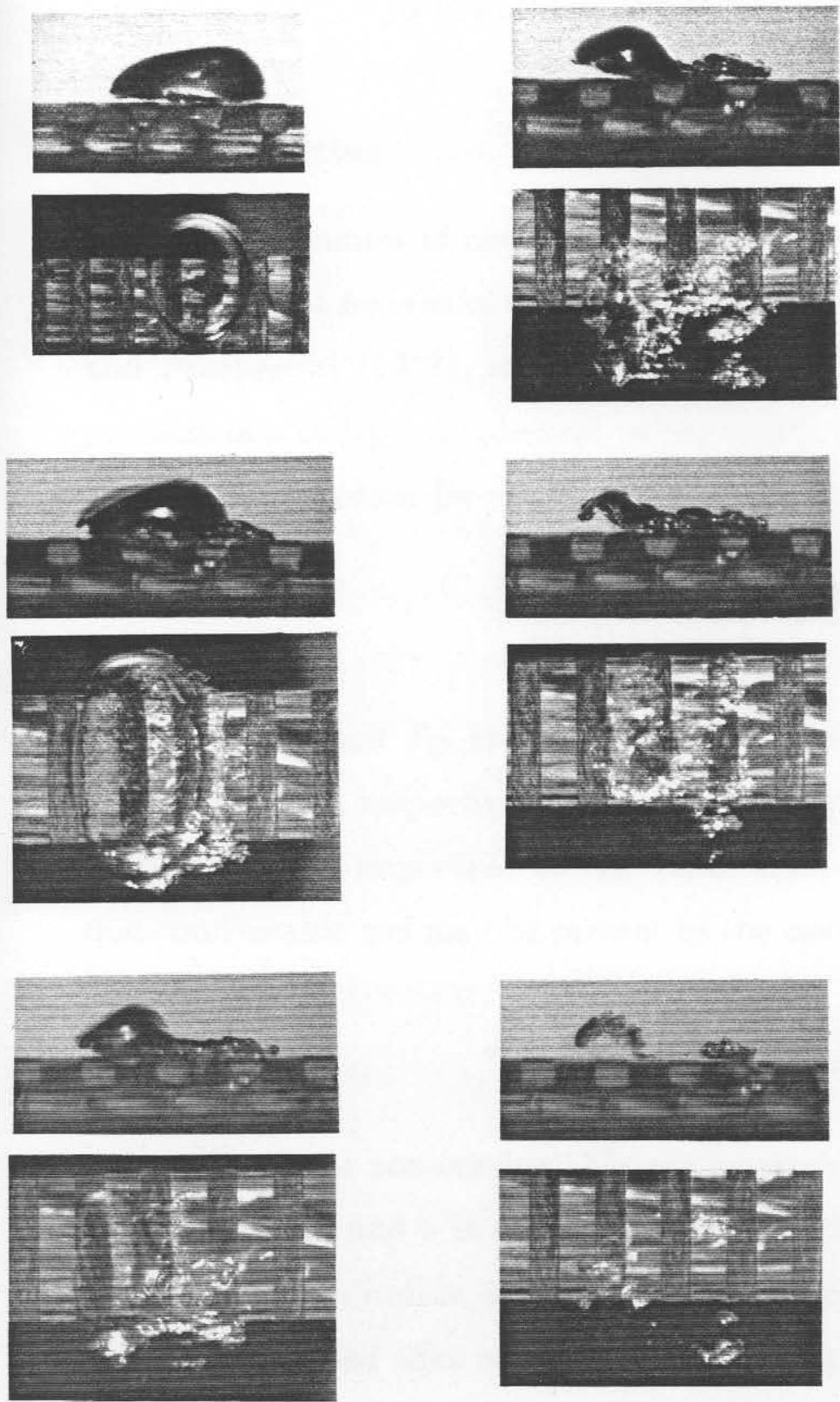
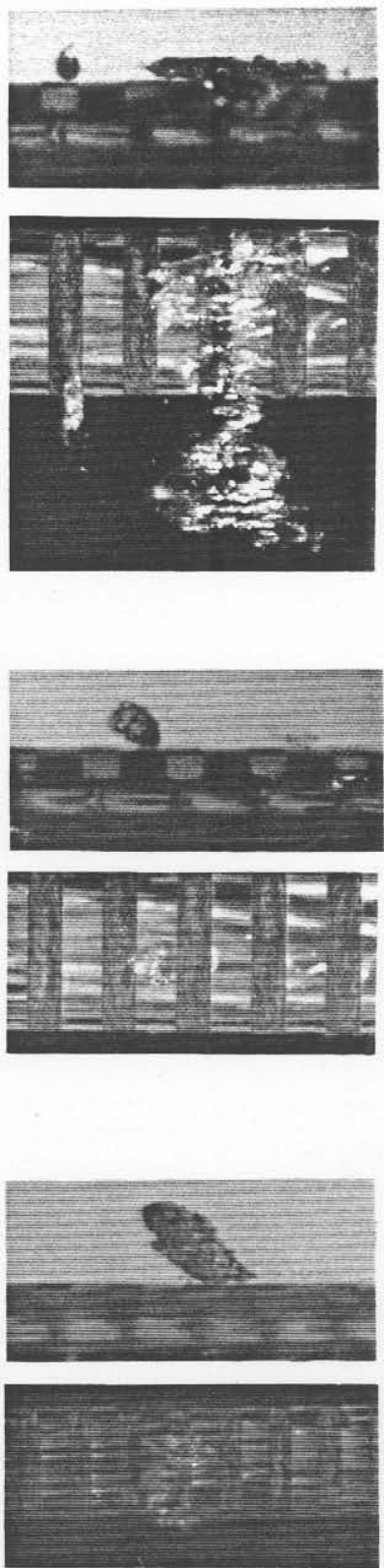

Figure 4.13 Series of photographs detailing typical bubble collapse mechanism on the I.T.T.C. headform, $U=8.7 \mathrm{~m} / \mathrm{s}$ and $\sigma=0.45$. 
CHAPTER 5

\section{Theoretical Analysis of the Dynamics of Single Bubble Cavitation}

\subsection{Introduction}

The dynamics of cavitation bubbles has been a topic of research for a long time. Excellent reviews of this subject have been presented by Flynn (1964), Plesset and Prosperetti (1977), and Blake (1986). Rayleigh (1917) first considered the problem of a collapsing spherical empty cavity, and he derived from the momentum equation the relation for the bubble boundary, $R(t)$ :

$$
R \frac{d^{2} R}{d t^{2}}+\frac{3}{2}\left(\frac{d R}{d t}\right)^{2}=\frac{P(R)-P_{O}}{\rho}
$$

where $P(R)$ and $P_{O}$ are the liquid pressure at the bubble wall and far from the bubble wall respectively, and $\rho$ is the liquid density. Viscosity and surface tension may be important at the vapor fluid interface of the bubble, and some non-condensable gas may be present in the cavity such that

$$
P(R)=P_{V}+P_{G}-\frac{2 S}{R}-\frac{4 \nu}{R} \frac{d R}{d t}
$$

where $P_{G}$ is the non-condensable gas pressure, $P_{V}$ is the vapor pressure, $S$ is the surface tension, and $\nu$ is the liquid viscosity. Note that $P_{G}$ is generally dependant upon the bubble radius, and for adiabatic processes, $P_{G}=P_{G O}\left(R_{O} / R\right)^{3 \gamma}$ where it has been assumed that no solution or dissolution of non-condensable gas occurs, maintaining a constant mass of gas in the cavity. The generalized form of the equation for the bubble wall known as the Rayleigh- Plesset equation may then be written as

$$
R \frac{d^{2} R}{d t^{2}}+\frac{3}{2}\left(\frac{d R}{d t}\right)^{2}=\frac{1}{\rho}\left[P_{V}+P_{G O}\left(\frac{R_{O}}{R}\right)^{3 \gamma}-P_{O}-\frac{2 S}{R}-\frac{4 \nu}{R} \frac{d R}{d t}\right]
$$


The significant nonlinearities of the Rayleigh-Plesset equation have made analytical solutions difficult to obtain, although some may be found for simple pressure histories, and asymptotic solutions may be derived for certain cases.

The Rayleigh-Plesset equation may be integrated numerically for specific bubble initial conditions and a known pressure history, and this was performed for comparison with the experimental data. Pressure distributions for the Schiebe body (Gates et al. (1979)) and the I.T.T.C. headform (Hoyt (1966)) presented in Figure 4.1 have been determined previously and were employed to construct the pressuretime history a nucleus would experience passing near the headform, assuming no slip between the bubbles and the liquid and a small offset from the stagnation streamline, and these pressure histories are shown in Figure 5.1. Calculations were performed with upstream nuclei of various sizes and with various free stream velocities, cavitation numbers, and offsets from the stagnation streamline. The viscosity, density, and surface tension, of water at $20^{\circ} \mathrm{C}$ were employed in evaluating these effects in the Rayleigh-Plesset solution. All the numerical results presented below are the results of these calculations.

\subsection{Bubble Dynamics}

\subsubsection{Bubble Stability}

Changes in the pressure difference across the bubble wall act as a driving force to changes in the bubble radius, and for small pressure perturbations, the $(d R / d t)^{2}$ term of Equation 5.3 is small. Then the bubble will respond quasi- statically or as a simple oscillator with natural frequency

$$
f=\frac{1}{2 \pi}\left(\frac{3 \gamma P_{G O}}{\rho R_{O}{ }^{2}}-\frac{2 S}{\rho R_{O}{ }^{3}}\right)^{1 / 2}
$$

However, if the pressure difference across the bubble wall becomes large, the $(d R / d t)^{2}$ term will dominate the behavior of the Rayleigh-Plesset equation, and the bubble may grow explosively to produce cavitation. 
Figure 5.2 provides an example of the calculated dependence of the maximum bubble radius on the original nucleus size for bubbles experiencing the pressure histories of the two headforms at different cavitation numbers. Note that nuclei below a certain size (which depends on the cavitation number) hardly grow at all while nuclei above the critical size grow to many times their initial size. This feature is predicted by the stability analysis of Johnson and Hsieh (1966). For a specific cavitation number and flow geometry, a bubble is statically unstable only if

$$
\frac{R_{L}}{R_{H}}>\frac{8}{3} \frac{S}{\rho R_{H} U^{2}} \frac{-1}{\left(\sigma+C_{P M}\right)}
$$

where $C_{P M}$ is the minimum pressure coefficient, and $R_{L}$ is the local bubble size. The computations show that so long as the bubble remains stable, then $R_{L}$ is generally in the range $R_{O}<R_{L}<2 R_{O}$. Consequently, the critical nuclei size, $R_{C}$, is approximately given by the modified stability criteria

$$
R_{C}>\frac{8}{3} \frac{\beta S}{\rho U^{2}} \frac{-1}{\left(\sigma+C_{P M}\right)}
$$

where $\beta$ is approximately 0.5 . The results of this expression are shown in Figure 5.3 along with data on the critical nuclei size obtained from the Rayleigh-Plesset calculations. Note that the higher the velocity, $U$, the smaller the critical radius, $R_{C}$, and therefore the larger the number of nuclei involved in cavitation.

The nature of nuclei stability has been extensively studied in the context of cavitation inception. Epstein and Plesset (1959) posed the "Bubble Paradox," noting that bubbles will generally not persist in a fluid but either rise to a free surface or dissolve under the action of surface tension. Consequently, researchers have introduced, among other effects, variable surface tensions to account for the persistence of nuclei (Parkin (1981)), and these models will have modified stability criteria. 


\subsubsection{Bubble Growth}

Figure 5.2 also reveals that the maximum volume that a cavitating nucleus may attain is principally a function of the minimum pressure the nucleus experiences along its trajectory. Once a nucleus begins to expand, its asymptotic growth rate is a strong function of the fluid pressure and only weakly influenced by the initial nuclei size. Consequently, all nuclei above the critical size travelling along the same trajectory will grow to approximately the same maximum size, as shown in the figure. This phenomena was observed experimentally, as mentioned in Section 4.2. While the nuclei size will range over almost three orders of magnitude, the observed bubble sizes ranged over less than one order of magnitude.

A rudimentary expression for the average bubble growth time, $\tau_{G}$, may be derived using asymptotic analysis of the Rayleigh-Plesset equation

$$
\tau_{G} \approx \frac{d}{U\left(1-C_{P A}\right)^{1 / 2}}
$$

were $d$ is the distance travelled by the bubble in the fluid region below vapor pressure, $C_{P A}$ is the average pressure coefficient in that region, and $U$ is the free stream velocity. This relation may be used to estimate the average maximum bubble radius, $R_{M}$

$$
R_{M} \approx d\left[\frac{1}{3} \frac{C_{P A}+\sigma}{C_{P A}-\sigma}\right]^{1 / 2}
$$

This formula for $R_{M}$ was found to underestimate the numerically-calculated mean maximum bubble radius by approximately seventy five percent. Consequently, the bubble growth rate may be better characterized by the minimum pressure coefficient rather than the average pressure coefficient.

\subsubsection{Bubble Collapse}

After leaving the region of low pressure, the bubble will collapse and may rebound if any non-condensable gas is present in the bubble. Rayleigh (1917) 
calculated the amount of time a bubble will take to collapse after experiencing a step function pressure rise from the bubble equilibrium pressure, assuming that the internal bubble pressure is constant (i.e., a cavity only composed of fluid vapor). The bubble will collapse with an asymptotic wall velocity given by

$$
\frac{d R}{d t} \approx U\left[\frac{1}{3}\left(C_{P C}+\sigma\right)^{1 / 2}{\frac{R_{M}}{R}}^{3 / 2}\right]
$$

and the total collapse time will be given by

$$
\tau_{C} \approx 0.65 \frac{R_{M}}{U}\left[\frac{1}{C_{P C}+\sigma}\right]^{1 / 2}
$$

where $C_{P C}$ is the average pressure coefficient in the region of bubble collapse with pressure $P_{C}$. This formula adequately predicts the collapse time of spherical and quasi-spherical bubbles generated is still fluid (Miles (1966)) or over an axisymmetric body (Plesset (1949)) even though the analysis predicts an infinite bubble wall velocity at zero radius.

As the cavity collapses, the bubble wall velocity increases exponentially, but the presence of any non-condensable gas in the cavity will ultimately cushion the bubble collapse until a minimum radius is reached, when the bubble may then rebound. Again, using asymptotic analysis for a step change in pressure from the bubble equilibrium pressure, the bubble minimum radius, $R_{m}$, that results may be derived. For the case of adiabatic compression,

$$
\frac{R_{m}}{R_{M}} \approx\left[\frac{1}{(\gamma-1)} \frac{P_{G M}}{P_{C}}\right]^{1 / 3(\gamma-1)}
$$

where $P_{G M}$ is the partial pressure of the non-condensable gas at the bubble maximum. The time scales of the bubble collapse are important in determining the spectral content of the bubble acoustic emission. Near the point of minimum 
radius, $R(t)$ may be described by the "rebound parabola" (Blake (1986))

$$
\frac{R-R_{m}}{R_{M}} \approx\left[\frac{\tau_{C}-t}{\delta \tau_{d R / d t=0}}\right]^{2}
$$

where $\delta \tau_{d R / d t=0}$ is the time interval between the points where $d R / d t=0$ near the minimum radius and is given by

$$
\delta \tau_{d R / d t=0} \approx \tau_{C}\left[\frac{2 P_{C}}{1.2 P_{G M}}\left(\frac{R_{m}}{R_{M}}\right)^{3 \gamma+2}\right]^{1 / 2}
$$

These relationships must be considered valid only for cases where the bubble wall velocity is much less then the sonic speed of the fluid, $c$, and near the final stages of collapse, this may not be the case. Gilmore (1952) modified the Rayleigh-Plesset equation to include liquid compressibility using the Kirkwood-Bethe approximation (Kirkwood and Bethe (1942)). This approximation assumes that the quantity $r\left(h+u^{2} / 2\right)$ is constant along outgoing characteristic, $d r=(u+c) d t$, where $h$ and $u$ are the local fluid enthalpy and velocity respectively, and that the fluid is baratropic. The Kirkwood-Bethe assumption is discussed at length by Cole (1948) and has been demonstrated to be valid during the bubble collapse, when an expansion wave is generated by the bubble (Flynn (1975)). The Gilmore equation for the bubble wall is

$$
\left(1-\frac{1}{C} \frac{d R}{d t}\right) R \frac{d^{2} R}{d t^{2}}+\frac{3}{2} \frac{d R^{2}}{d t}\left(1-\frac{1}{3 C} \frac{d R}{d t}\right)=H\left(1+\frac{1}{C} \frac{d R}{d t}\right)+\left(1-\frac{1}{C} \frac{d R}{d t}\right) \frac{1}{C} \frac{d R}{d t} \frac{d H}{d t}
$$

where $C$ and $H$ are the sonic speed and enthalpy at the bubble wall. A more recent evaluation of this approach can be found in Lezzi and Prosperetti (1986 and 1987).

Hickling and Plesset (1964) and Ivany and Hammitt (1966) used Gilmore's equation to numerically generate solutions for the collapse of a bubble that contained non-condensable gas. In both cases, a shock wave was generated near the point of bubble rebound, and the shock strength and bubble minimum radius was strongly influenced by the amount of non-condensable gas present in the cavity. 


\subsection{Acoustic Emission of Cavitating Bubbles}

\subsubsection{General Considerations}

Travelling bubble cavitation noise is caused by the rapid changes in bubble volume over entire bubble trajectory. Noise produced by volume changes may generally be described by the acoustic emission of a monopole source where the radiated acoustic pressure, $P_{A}$, is given by

$$
P_{A}(r, t)=\frac{\rho}{4 \pi r} \frac{d^{2} V}{d t^{2}}
$$

where $V(t)$ is the volume of the bubble and $r$ is the distance from the center of the bubble to the point of measurement. This relationship is valid in the acoustic far field and for subsonic bubble wall velocities. Analytical and numerical solutions of the Rayleigh-Plesset equation may be used to find $d^{2} R / d t^{2}$ to calculate the resulting noise emission.

\subsubsection{Emission During Bubble Collapse}

Although some noise will be produced over the entire trajectory of the bubble, the majority of the acoustic energy is emitted during the violent collapse and rebound. The Rayleigh-Plesset equation is valid for the entire region of bubble collapse except for an interval around the time of minimum radius when large bubble wall velocities can make liquid-compressiblity effects important.

The shock wave generated by a bubble collapse has been studied by several researchers. Baiter (1974) used the Rayleigh-Plesset equation in a quasi-compressible analysis to determine the acoustic pressure generated by the adiabatic collapse of a gas- filled bubble. A shock wave of the form

$$
P_{A}(t)=P_{S} e^{-t / \theta}
$$


was assumed a priori, and then $P_{S}$ and $\theta$ identified as

$$
P_{S}=\frac{1}{2} \rho c^{2} \frac{R_{M}}{r}\left[\frac{P_{G M}}{\rho c^{2}}\right]^{1 / 4}\left[\ln \frac{r}{R_{m}}\right]^{-1 / 2}
$$

and

$$
\theta=4.4 \frac{R_{M}}{c}\left[\frac{P_{G M}}{\rho c^{2}}\right]^{1 / 4}\left[\ln \frac{r}{R_{m}}\right]^{1 / 2}
$$

where

$$
R_{m}=R_{M}\left[\frac{3 P_{G M}}{P_{C}+3 P_{G M}}\right]
$$

with $\gamma=3 / 4$ and $P_{G M} \ll P(R)$.

Esipov and Naugol'nyhk (1973) used Gilmore's equation and the KirkwoodBethe approximation, along with the above assumptions, to derive the following relationships for $P_{S}$ and $\theta$ :

$$
P_{S}=\frac{1}{\sqrt{2}} \rho c^{2} \frac{R_{M}}{r}\left[\frac{P_{G M}}{\rho c^{2}}\right]^{1 / 4}\left[\ln \frac{r}{R_{m}}\right]^{-1 / 2}
$$

and

$$
\theta=\frac{R_{M}}{c}\left[\frac{P_{G M}}{\rho c^{2}}\right]^{1 / 4}\left[M^{-3 / 2}+2 \sqrt{2}\left[\ln \frac{r}{R_{m}}\right]^{1 / 2}\right]
$$

where

$$
R_{m}=R_{M} M^{-1 / 2}\left[\frac{P_{G M}}{\rho c^{2}}\right]^{1 / 4}
$$

and

$$
M=\left[\frac{1}{2}+\frac{3 P_{G M}^{3 / 4}\left(\rho c^{2}\right)^{1 / 4}}{P_{C}}\right]^{-1}
$$

for cases where $r \gg R_{m} \exp \left(M^{3 / 4}\right)$ and $P_{S} \gg \rho c^{2} / 7$ for voids in water. Ellis (1966) has reviewed the experimental observation of shock waves resulting from bubble collapse.

\subsection{Spectral Analysis of Bubble Acoustic Emission}

Fitzpatrick and Strasberg (1956) introduced the first spectral analysis of cavitation bubble noise. In their analysis, each stage of the bubble growth and 
collapse history was analyzed separately and the results combined to arrive at the total acoustic spectrum. Almost all of the acoustic energy radiated occurs during the bubble collapse, and most of this energy will be in the frequency range greater than $f \tau_{C}>1$. A detailed treatment of this subject is presented by Blake (1986).

One part of the bubble collapse may be described by a constant bubble wall velocity, as in Equation 5.9. By taking the Fourier transform of this relation, the generated acoustic energy has a predicted frequency dependence approximated by $S_{P} \approx f^{-2 / 5}$. Since the acoustic energy released by the bubble is finite, an upper frequency limit is expected, and this limit may be inferred for the case of an incompressible fluid by the rebound time scale given by Equation 5.13. The high frequency limit would occur near frequencies on the order of $f \delta \tau_{d R / d t=0}>1$, and the spectral amplitudes would decay at a rate of $S_{P} \approx f^{-10}$. However, fluid compressibility will lower the high-frequency cutoff.

Fluid compressibility results in the formation of a shock wave near the point of rebound (Kimoto (1987)), and for an exponential shock wave described in Equation 5.16 , the acoustic spectrum will be given by

$$
S_{P}=\frac{\left(P_{S} \theta\right)^{2}}{1+(2 \pi \theta f)^{2}}
$$

In this model, the shock wave time constant, $\theta$, will determine the high frequency cutoff, and estimates of this value may be taken from Equations 5.18 or 5.21.

\subsection{Conclusions}

The above analysis aids in the interpretation of actual cavitation dynamics and noise emission. Effects such as gas diffusion, viscosity, and thermal energy transfer could also be considered, and the stability of the bubble vapor-fluid interface could be analyzed. However, previous researchers have noted that only rarely will cavitation bubbles remain spherical when they collapse near a solid boundary. In the present study, the cavitation bubbles are clearly non- spherical, and the 
collapse mechanisms described in Chapter 4 are very complex. Consequently, the above analysis must be viewed as a rough approximation to the behavior of actual cavitation bubbles. 


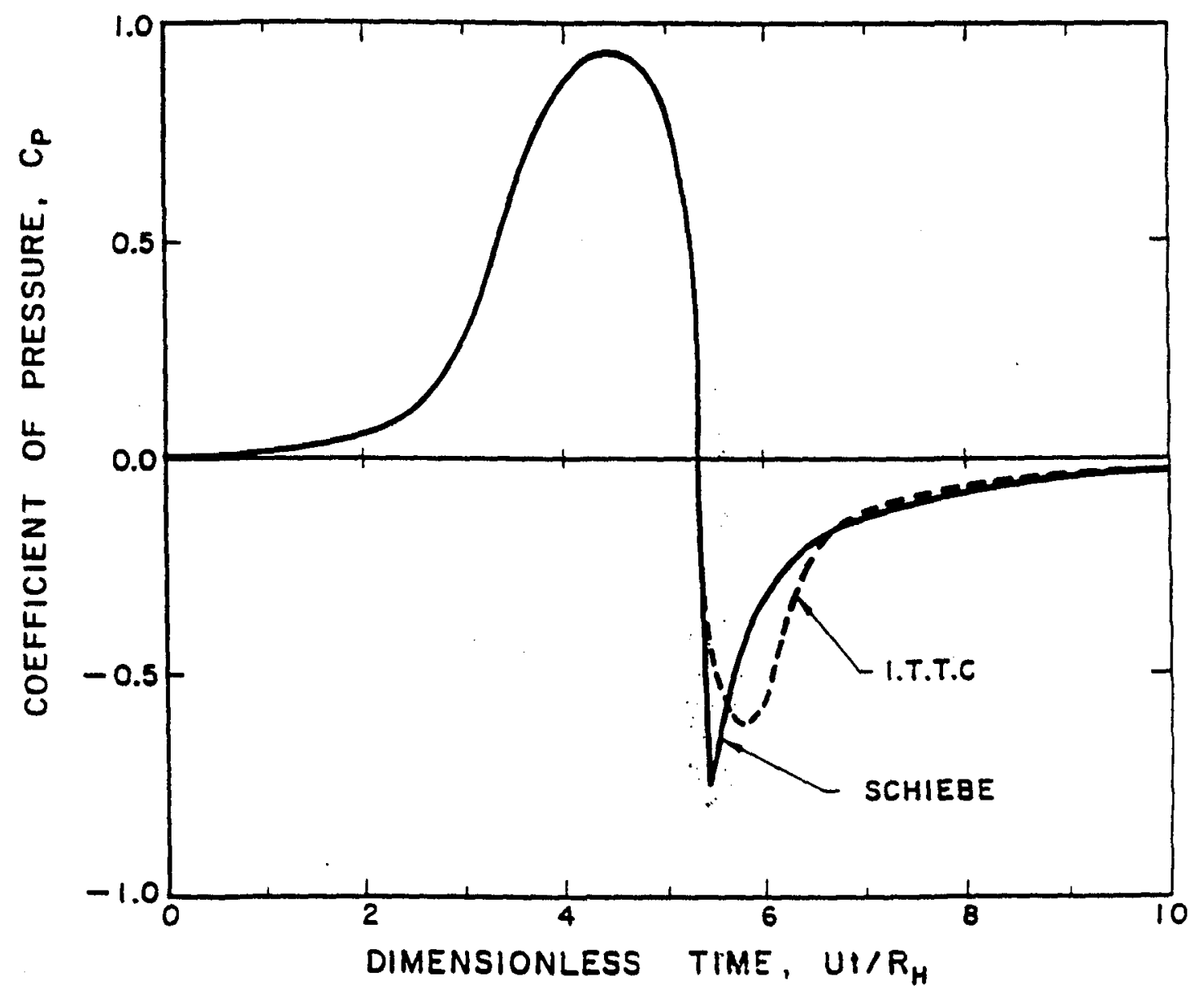

Figure 5.1 Pressure history a nucleus would experience passing near the Schiebe or I.T.T.C. headform. 


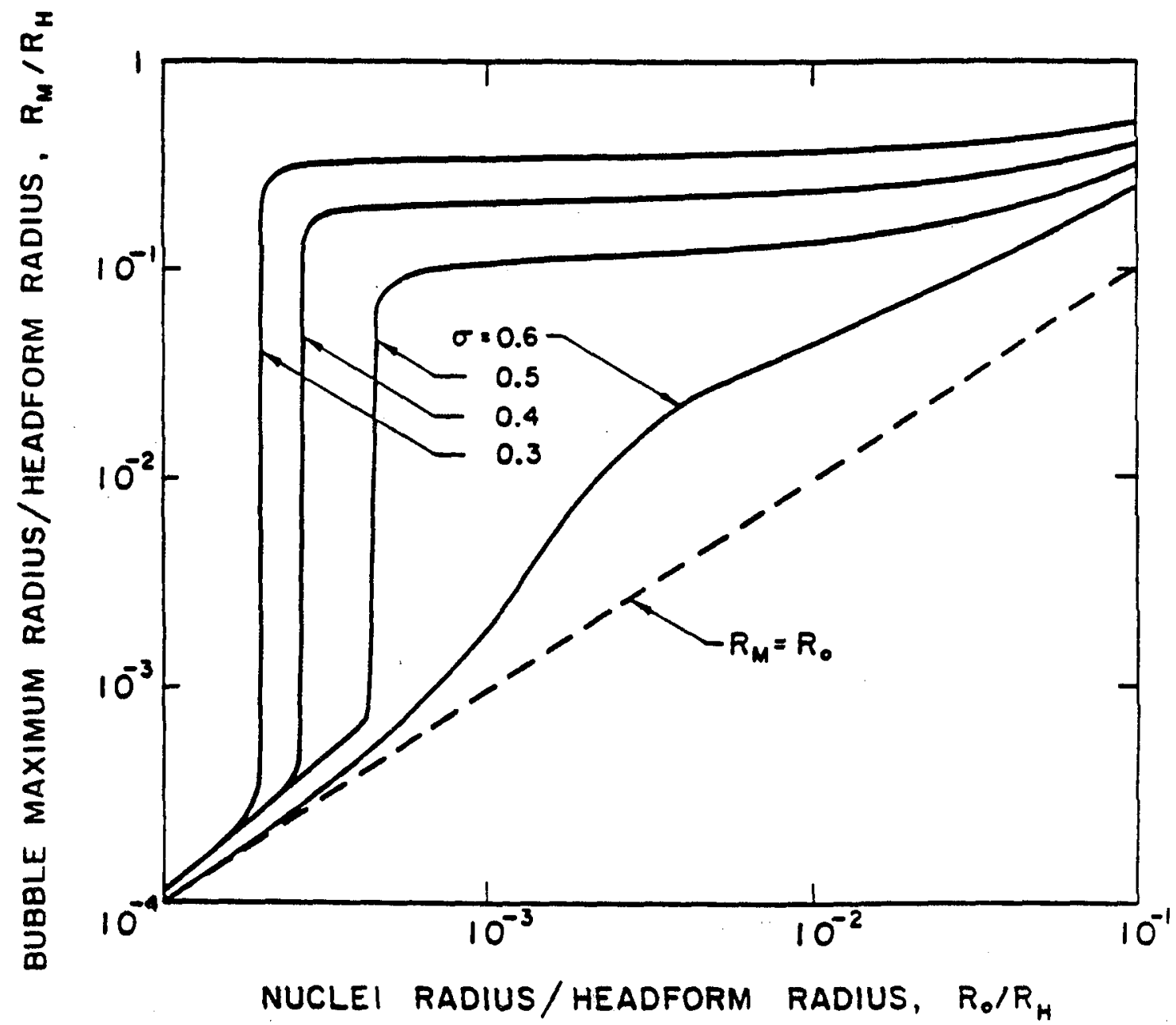

Figure 5.2 Numerical calculation of the bubble maximum radius as a function of nucleus radius for nuclei passing near the I.T.T.C. headform. 


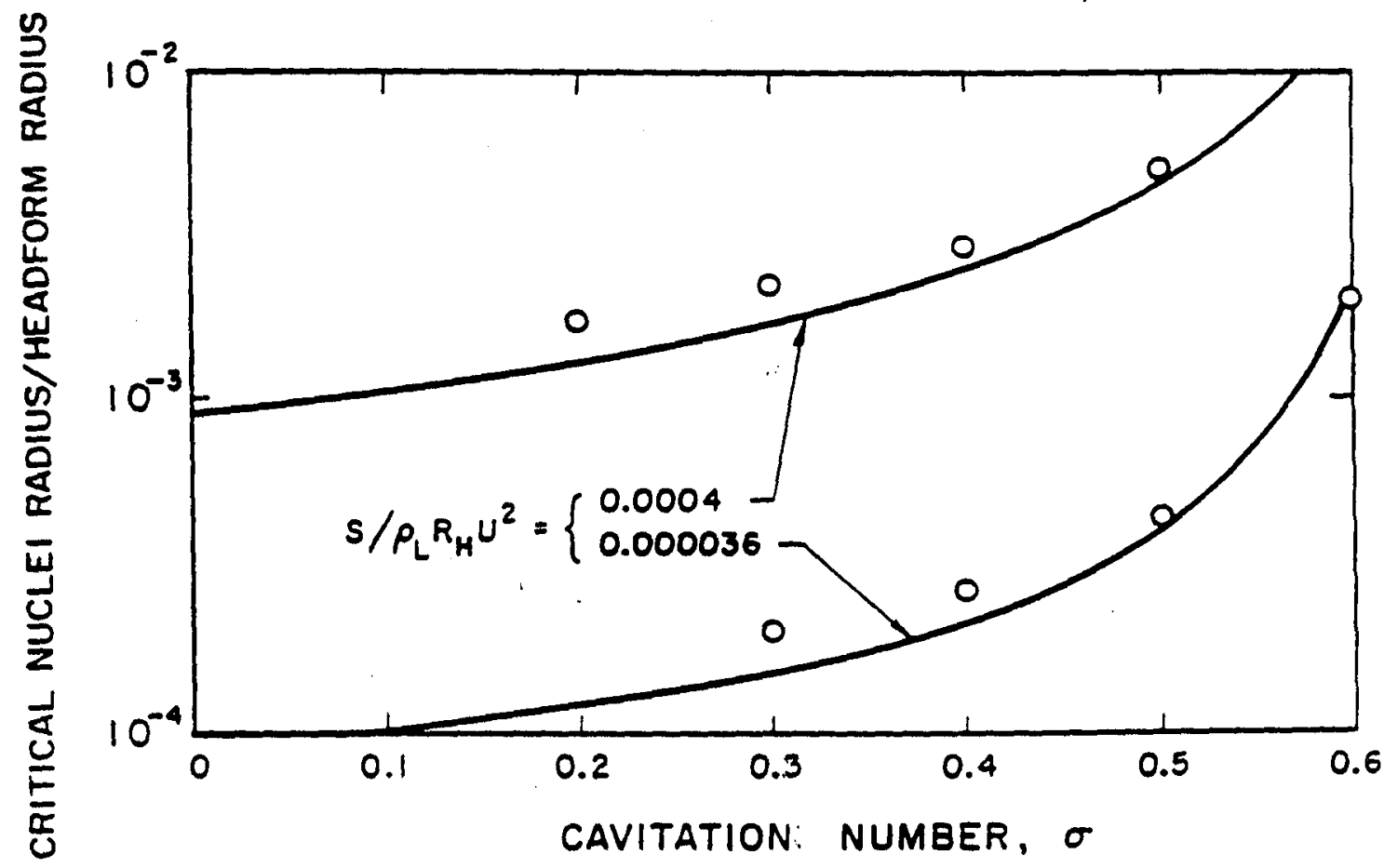

Figure 5.3 Crital nuclei radius as a function of flow parameters for nuclei passing near the I.T.T.C. headform. 


\section{CHAPTER 6}

\section{Measurement of the Acoustic Emission of Single Cavitation Bubbles}

\subsection{Introduction}

The detailed relationship between the collapse mechanism of hydrodynamic cavitation bubbles and the resulting noise generation is not completely clear, but some features are suggested by the photographs. First, as other investigators have concluded (for example Harrison (1952) and Chahine, Courbiere, and Garnaud (1979)), the majority of the noise is generated by the violence of the first collapse; the growth phase contributed no measurable noise signal. The rebound produces a rough bubble that may also collapse to produce a noise pulse of lesser magnitude. However, noise was not necessarily generated by every bubble collapse. Smaller bubbles would often collapse without an acoustic pulse, and larger bubbles would sometimes produce a muted collapse. Figure 6.1 is an example of a typical noise pulse. The first large noise spike is the emission of the first collapse, and the second is the noise generated by the bubble rebound. Note the reverberant noise induced in the tunnel test section after the first collapse.

Figure 6.2 presents two magnified examples of the initial noise pulse generated by the collapse of a bubble on the I.T.T.C. headform. The first pulse has only one peak, but the second trace is an example of a multiple peak event. Multiple peaks suggest bubble fission prior to collapse, and the photographs presented in Chapter 4 reveal that many bubbles have undergone fission.

As the analysis in Chapter 5 indicates, the behavior of the cavitation bubble near the point of bubble minimum radius will be influenced by factors such as the non- condensable gas in the cavity and fluid compressibility, and non-spherical collapse mechanisms will affect the acoustic energy released. Although some researchers have used the peak acoustic pressure to characterize cavitation noise in- 
tensity (e.g., Van der Meulen (1989)), in this study the magnitude of acoustic pulses will be characterized by the acoustic impulse defined as

$$
I=\int_{t_{1}}^{t_{2}} P_{A} d t
$$

The points $t_{1}$ and $t_{2}$ were chosen to exclude the shallow pressure rise before collapse and the reverberation produced after the collapse (see Figure 6.2). The impulse is directly related to the acoustic energy emitted during the bubble collapse, and this quantity will be correlated with the maximum volume of the bubbles, a measure of the bubble's stored energy before collapse.

\subsection{Schiebe Body Results}

Experimental results for the relation between the impulse and the maximum bubble size on the Schiebe body are shown in Figures 6.3 to 6.6 for cavitation numbers of $\sigma=0.42,0.45,0.50$ and 0.42 at a tunnel velocity of $U=9 \mathrm{~m} / \mathrm{s}$. The data all appear to lie below an envelope that passes through the origin. The existence of this well-defined impulse envelope suggests that a collapsing bubble can generate, for a certain maximum volume, a specific impulse if it collapses in some particular but unknown way. It can, however, produce less than this maximum impulse if it collapses in other ways.

The different symbols represent the varying number of acoustic peaks that are generated upon collapse. As shown in Figure 6.3, the probability that a collapse will produce multiple peaks increases for larger bubbles. Yet, even as the number of peaks increases, the impulse often reaches its maximum possible value implying that, in some collapse mechanisms, fission does not decrease the total stored energy available to produce noise. Other large bubbles collapse to produce almost no acoustic impulse. The production of noise upon collapse is the result of violent changes in bubble volume near the point of minimum bubble volume, but larger bubbles may be sheared apart and dissipate thus losing their organized shape and 
preventing a coherent and concentrated collapse. Furthermore, larger bubbles may contain more undissolved gas (as a result of diffusion) and this would cushion the collapse and reduce the acoustic emission.

At higher cavitation numbers such as those in Figure 6.5, the number of larger bubbles is reduced, and most bubbles collapse to produce only one peak. However, a large number of very small bubbles will collapse and produce no noise at all, and these cases are represented by the "0" symbols. Mute events are generally not examples of "pseudo-cavitation" as observed by Dreyer (1987), but distinct cavitation events with a near-silent collapse mechanism.

Figures 6.7 to 6.10 display the averaged data from Figures 6.3 to 6.6. The symbols are the mean impulse produced by the cavitation events with maximum volume for a given volume bin. Each bin has at least ten events, and the bars represent the standard deviation of the impulse. The trends discussed above are clearly evident. For smaller volumes the relationship between the impulse and the maximum volume is reasonably determinant, but for bubbles of volume greater than approximately $40 \mathrm{~mm}^{3}$, the resulting impulse becomes more uncertain. Also, the slope of the impulse- maximum volume curves are approximately equal for the two cases shown, with the difference being due primarily to slight changes in the location of the maximum volume as the cavitation number changes.

\subsection{I.T.T.C. Body Results}

Results from the I.T.T.C. body are shown in Figures 6.11 to 6.14 for cavitation numbers $\sigma=0.45,0.48,0.50$ and 0.53 for $U=8.7 \mathrm{~m} / \mathrm{s}$, and the averaged data are presented in Figures 6.15 to 6.18. The general trends described above for the Schiebe body are also evident in these data. Significantly, however, the average impulse generated by the I.T.T.C. bubbles is about three times larger than that from the Schiebe body. This will be discussed further below.

Figure 6.19 is an example of data from the I.T.T.C. body taken near the 
attached cavitation formation index at $\sigma=0.42$ at $U=8.7 \mathrm{~m} / \mathrm{s}$, and Figure 6.20 is the averaged data. The impulses generated by smaller bubbles is much more uncertain, and, for many larger bubbles, no sound is generated at all. Since these larger bubbles generally have trailing streamers, the streamers seem to interfere with their collapse in a way that decreases or eliminates the noise generated upon collapse.

The average number of peaks for a given average diameter is plotted in Figure 6.21 for both headforms. The trends noted above are evident. For smaller bubbles, the average is less than unity, reflecting the influence of muted bubbles, and for larger bubbles, multiple peaking produced an average above unity. For the case of the I.T.T.C. body, however, the influence of the trailing streamers is noted by the reduced average number of peaks for the data set with the largest average volume. This data set occurs at the lowest cavitation number, near the attached cavitation inception point.

\subsection{Comparison with Numerical Results}

Acoustic pressure pulses resulting from the collapse of various sized bubbles were calculated in the manner described in Chapter 5, and acoustic impulses, $I$, were obtained by integration according to Equation 6.1 where $t_{1}$ and $t_{2}$ were taken to be the times when $d^{2} V / d t^{2}=0$ on either side of the first collapse. For those nuclei that become unstable and explosively cavitate the non-dimensional impulse, $I^{*}$, is defined as

$$
I^{*}=\frac{4 \pi I}{\rho R_{H} U}
$$

where we have assumed $r=R_{H}$ since this is the location of the hydrophone in the experiments.

The impulse $I^{*}$ is plotted in Figure 6.22 against the maximum volume of the bubbles non-dimensionalized by $R_{H}{ }^{3}$. A number of investigators (i.e., Fitzpatrick and Strasberg (1956) and Hamilton, Thompson, and Billet (1982)) have suggested 
that the magnitude of the acoustic signal should be related to the maximum size of the bubble, and this is born out in Figure 6.14 where the data for a range of cavitation numbers and two Weber numbers, $W e$, are contained within a fairly narrow envelope.

The median line was converted to dimensional values and is plotted in Figure 6.23 where it is compared with data sets from the Schiebe and I.T.T.C. experiments. It is striking to note that the envelope of the maximum impulse from the experiments is within a factor of two of the Rayleigh-Plesset calculation for the I.T.T.C. body and within a factor of six for the Schiebe body. This suggests that, despite the departure from the spherical shape during collapse, the Rayleigh-Plesset solutions come close to predicting the magnitude of the noise impulse generated by individual bubbles.

It is not surprising that the predicted impulse is greater than those determined experimentally. In fact, the theoretical impulse may be considered the maximum impulse possible for a given bubble volume since a spherically symmetric collapse mechanism employed in the calculation is probably the most efficient noise producing mechanism. The difference between the measured impulses and the theoretical impulse is an indication of the inefficiency of the actual collapse mechanism.

Since the average impulses are closer to the theoretically predicted values for the I.T.T.C. body than for the Schiebe body, the I.T.T.C. collapse mechanism is considered more efficient. In fact, collapsing bubbles on the I.T.T.C. headform generally produce vapor tubes further above the body surface than those formed by collapsing bubbles over the Schiebe body. The I.T.T.C. vapor tube collapse is therefore less influenced by the body surface and consequently leads to a more compact collapse mechanism and more efficient acoustic emission.

This concept of collapse efficiency may be compared with Baiter's (1986) idea of the acoustic efficiency of a collapsing bubble. His acoustic efficiency is defined as 
the ratio of the acoustic energy emitted by the bubbles to the total energy stored in the bubble at the point of its maximum volume. Hentschel and Lauterborn (1982) showed experimentally that spherical bubbles collapsing in an unbounded fluid lost only a small portion of their total stored energy through acoustic emission. Consequently, the definition of efficiency used in the current work is the ratio of the acoustic energy emitted by a bubble of volume $V_{B}$ to the maximum possible acoustic energy emitted from a spherical bubble of volume $V_{B}$ collapsing in an unbounded fluid.

The duration of the impulse (as opposed to the magnitude) is much better understood. Here, the duration, $T$, is defined as the time between the points of zero acoustic pressure prior to and after the first collapse. This time is simply related to the total collapse time defined in Equation 5.10, which is used by many authors (e.g., Blake, Wolpert, and Geib (1977) and Arakeri and Shanmuganathan (1985)). Like the collapse time, it will be approximated by

$$
T^{*}=\alpha \frac{R_{M}}{U}\left(\frac{2}{\sigma}\right)^{1 / 2}
$$

where $\alpha$ is some constant of order unity. It follows that the dimensionless impulse duration $T^{*}=T U / R_{H}$ should be primarily a function only of $R_{M} / R_{H}$, and this is confirmed by the Rayleigh-Plesset solution, the results for which are shown in Figure 6.24. Also plotted are typical experimental data from the Schiebe body. Note that the numerical results lie within a narrow envelope for varying cavitation numbers and that the slope of the narrow envelope is close to unity. The experimental data is about one third the predicted magnitude. Note, however, that the definitions of $t_{1}$ and $t_{2}$ are somewhat arbitrary.

\subsection{Spectral Analysis of Bubble Emission}

Figure 6.25 spectra derived from the experimental data. A series of individual pressure pulses were recorded at the specified velocity and cavitation number and 
Fourier transformed. The resulting spectra were averaged to produce the composite spectra in the figure; the signals were not altered to remove the effects of tunnel reverberation. Such a composite spectrum will be equivalent to the spectrum derived from a measurement of a long series of cavitation noise pulses, if the cavitation events occur randomly. This phenomenon will be further detailed in Chapter 7.

The measured spectral shape varies little with cavitation number, only the overall spectral magnitudes change. A decrease of approximately $-12 d B /$ decade is noted until about $100 \mathrm{kHz}$ where a sharp falloff occurs. This cut- off frequency corresponds to the frequency response limit of the hydrophone.

Asymptotic analysis of the Rayleigh-Plesset equation predicts a spectral shape of $S_{P} \approx f^{-2 / 5}$ for frequencies of $10 \mathrm{kHz}$ to $100 \mathrm{kHz}$ until a high frequency roll-off. The experimental spectrum has a shape of approximately $S_{P} \approx f^{-3 / 5}$, which is similar but not identical to the predicted trend. Hamilton (1981), however, observed an almost completely flat spectrum in this range based on his integral measurement of bubble cavitation noise.

Of particular interest is the high frequency limit of cavitation noise. For collapse in an incompressible fluid, the high frequency limit occurs at frequencies given approximately by $f \delta \tau_{d R / d t=0}>1$. For typical values of the partial pressure of the gas in the bubble, this implies cutoff frequencies on the order of 1 to $5 \mathrm{MHz}$ (Blake (1986)) in the current experiment. However, fluid compressibility will further decrease the noise bandwidth.

As bubble wall velocities approach the sonic speed of the fluid, an expansion wave will be formed by the bubble until the point of rebound, when a compression wave will be emitted. This shock wave can be approximated by an exponential pressure pulse defined by Equation 5.16 (Mellen (1956)). The spectrum of such a pulse would be flat until the cutoff frequency, where the spectrum would roll off at 
a rate of $S_{P} \approx f^{-2}$. The cut off frequency is determined by the shock wave time constant, $\theta$, which is strongly dependant upon the amount of non-condensable gas present in the bubble. This frequency has been estimated to be between $50 \mathrm{kHz}$ and $300 \mathrm{kHz}$ (Hamilton (1981)). Since the measured spectrum does not roll off before the hydrophone frequency limit, it is possible that the acoustic cutoff frequency is greater than $100 \mathrm{kHz}$. Hamilton (1981) and Barker (1975) spectrally analyzed integrally measured travelling bubble cavitation noise, and these spectra also failed to roll off at frequencies below $100 \mathrm{kHz}$.

\subsection{Conclusions}

The acoustic emission of travelling cavitation bubbles may be related to the bubble trajectory and collapse mechanism, although this relationship is not well understood. The interaction of bubbles with the viscous flow near a surface will influence the noise produced by a bubble. Bubbles may fission to produce multiple collapses and pressure pulses, and local flow disturbances may induce collapse mechanisms that reduce or eliminate the potential acoustic emission.

Measured acoustic impulses compare well with the numerical predictions, even though the numerical model does not take into account fluid compressibility effects. The emission of a spherically symmetric collapse may be considered the most efficient noise producing mechanism for a given bubble volume. The actual impulses were between a third to a half of that predicted for the spherical case.

Individual noise pulses were spectrally analyzed and a composite spectrum was produced. Predicted trends in the spectrum were not readily recognizable, and a high frequency cutoff was not observed within the dynamic range of the acoustic measuring equipment. More refined acoustic measurements are needed to determine the exact nature of the pulse shape and resulting spectral emission. 


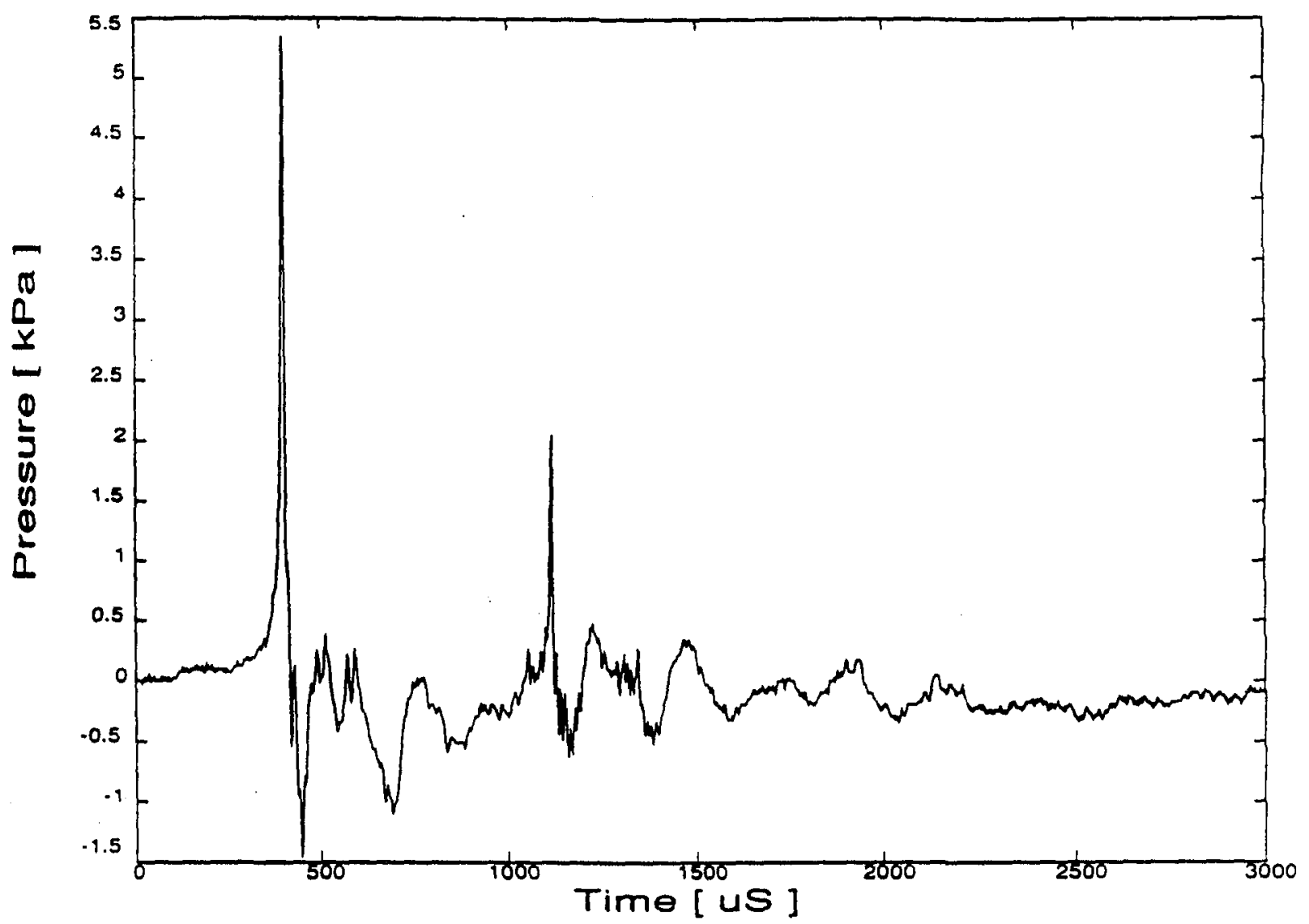

Figure 6.1 Typical cavitation bubble noise emission. The bubble was generated on the Schiebe body at $U=9 \mathrm{~m} / \mathrm{s}$ and $\sigma=0.45$ 

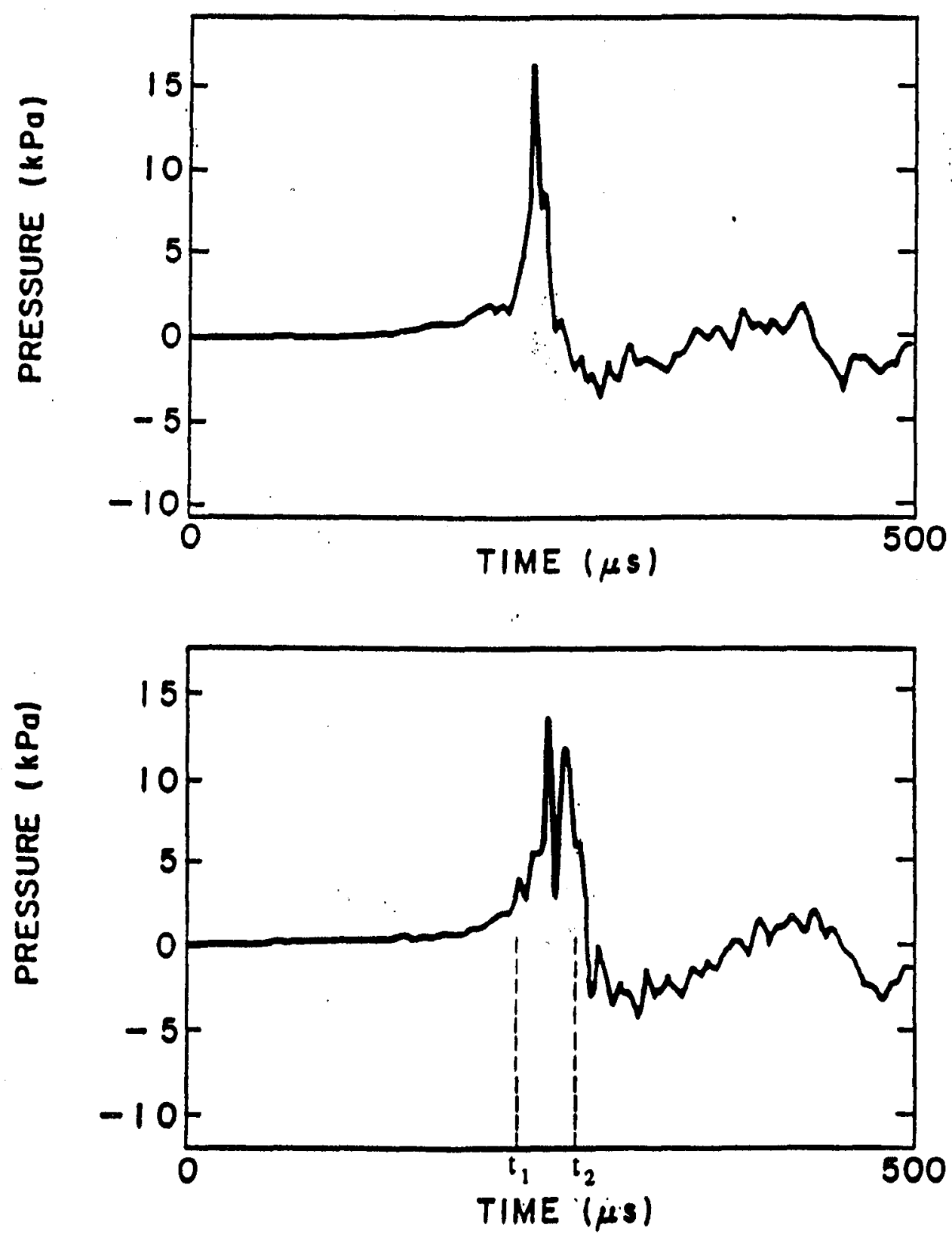

ACOUSTIC IMPULSE, $I=\int_{t_{2}}^{t_{1}} p_{A} d t$

Figure 6.2 Two examples of typical cavitation initial noise pulses. The bubbles were generated on the I.T.T.C. headform at $\sigma=0.45$ and $U=8.7 \mathrm{~m} / \mathrm{s}$. 


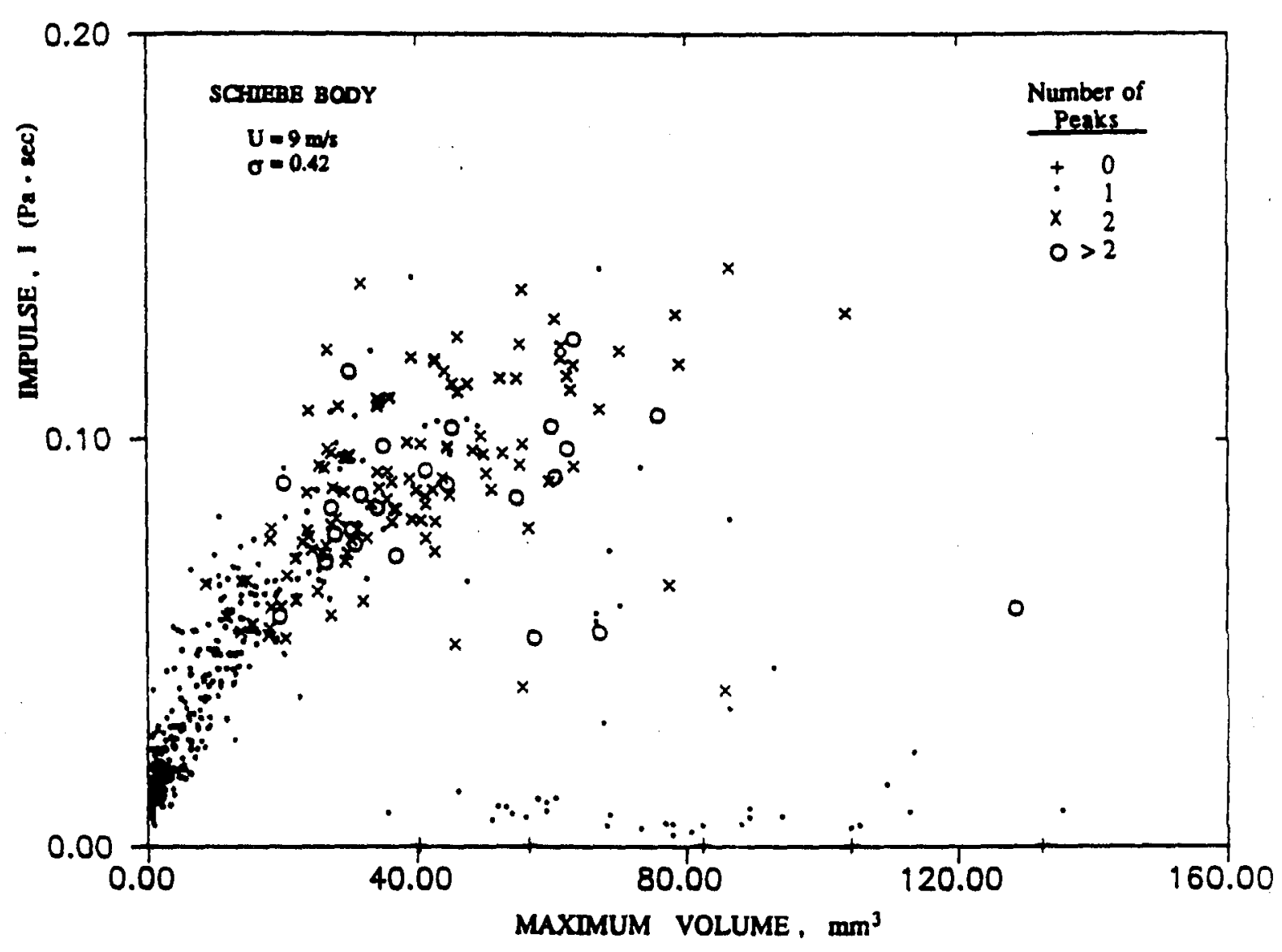

Figure 6.3 Impulse v. maximum bubble volume for Schiebe body, $U=9 \mathrm{~m} / \mathrm{s}$, $\sigma=0.42$. 


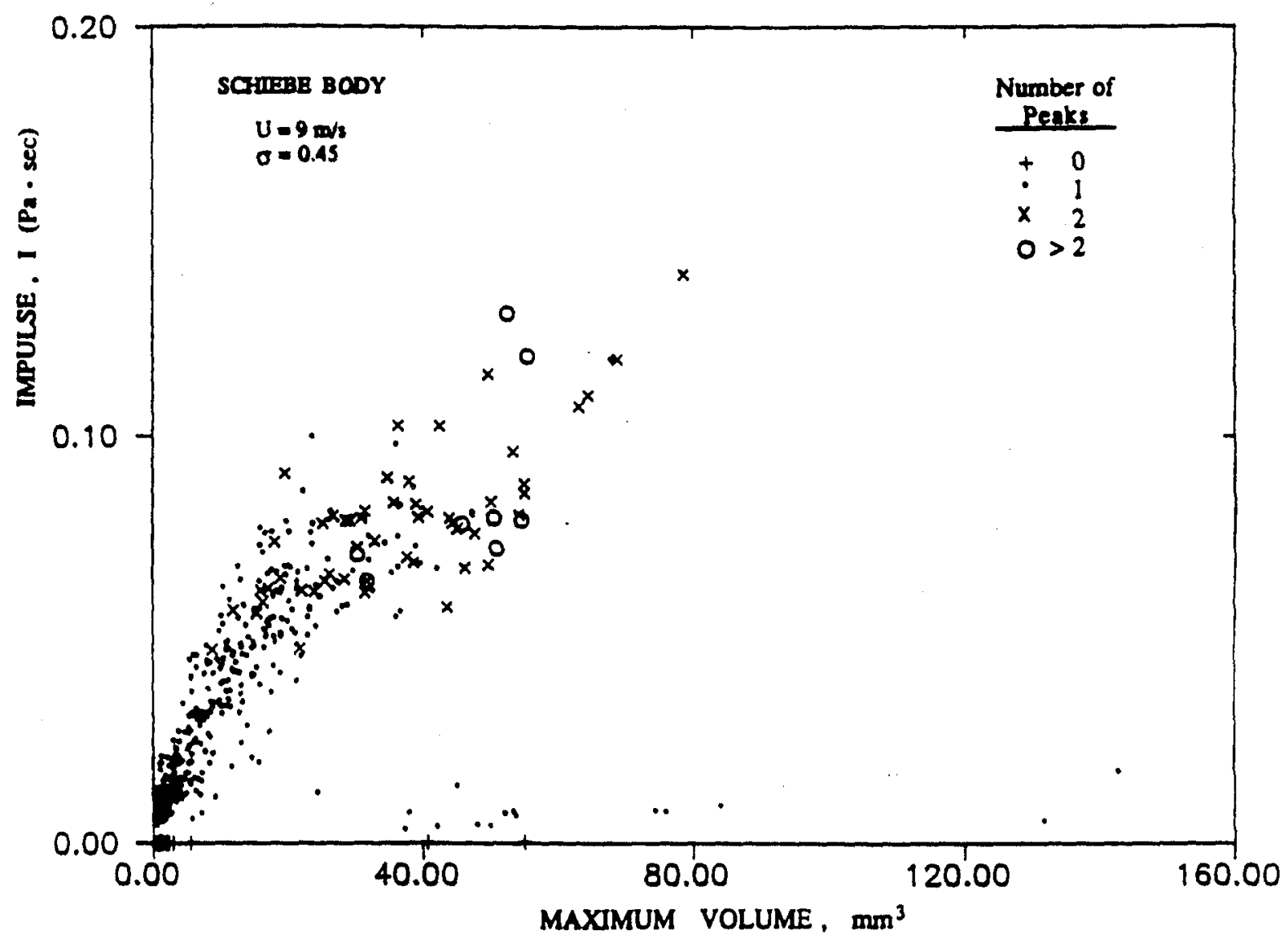

Figure 6.4 Impulse v. maximum bubble volume for Schiebe body, $U=9 \mathrm{~m} / \mathrm{s}$, $\sigma=0.45$. 


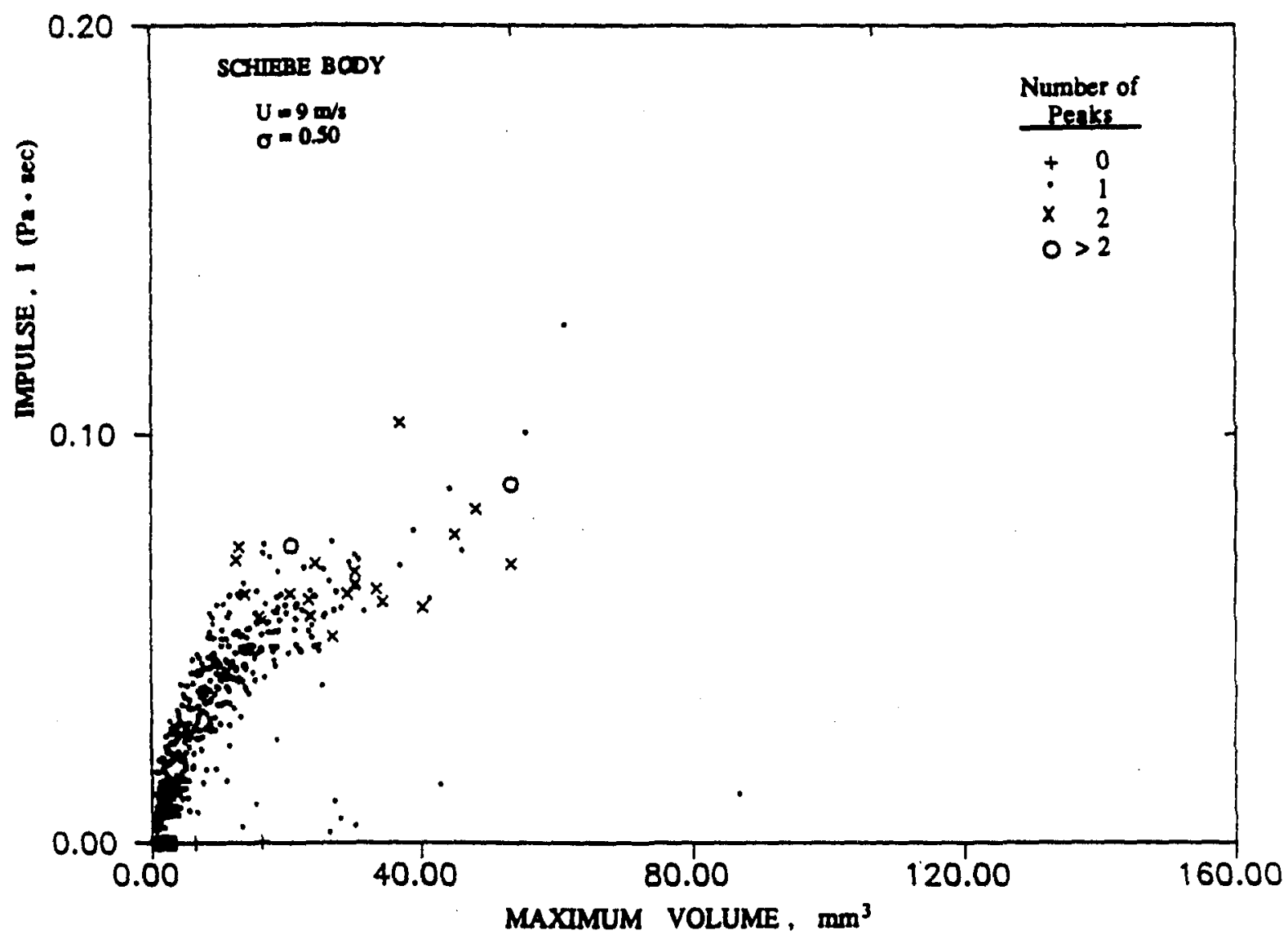

Figure 6.5 Impulse v. maximum bubble volume for Schiebe body, $U=9 \mathrm{~m} / \mathrm{s}$, $\sigma=0.50$. 


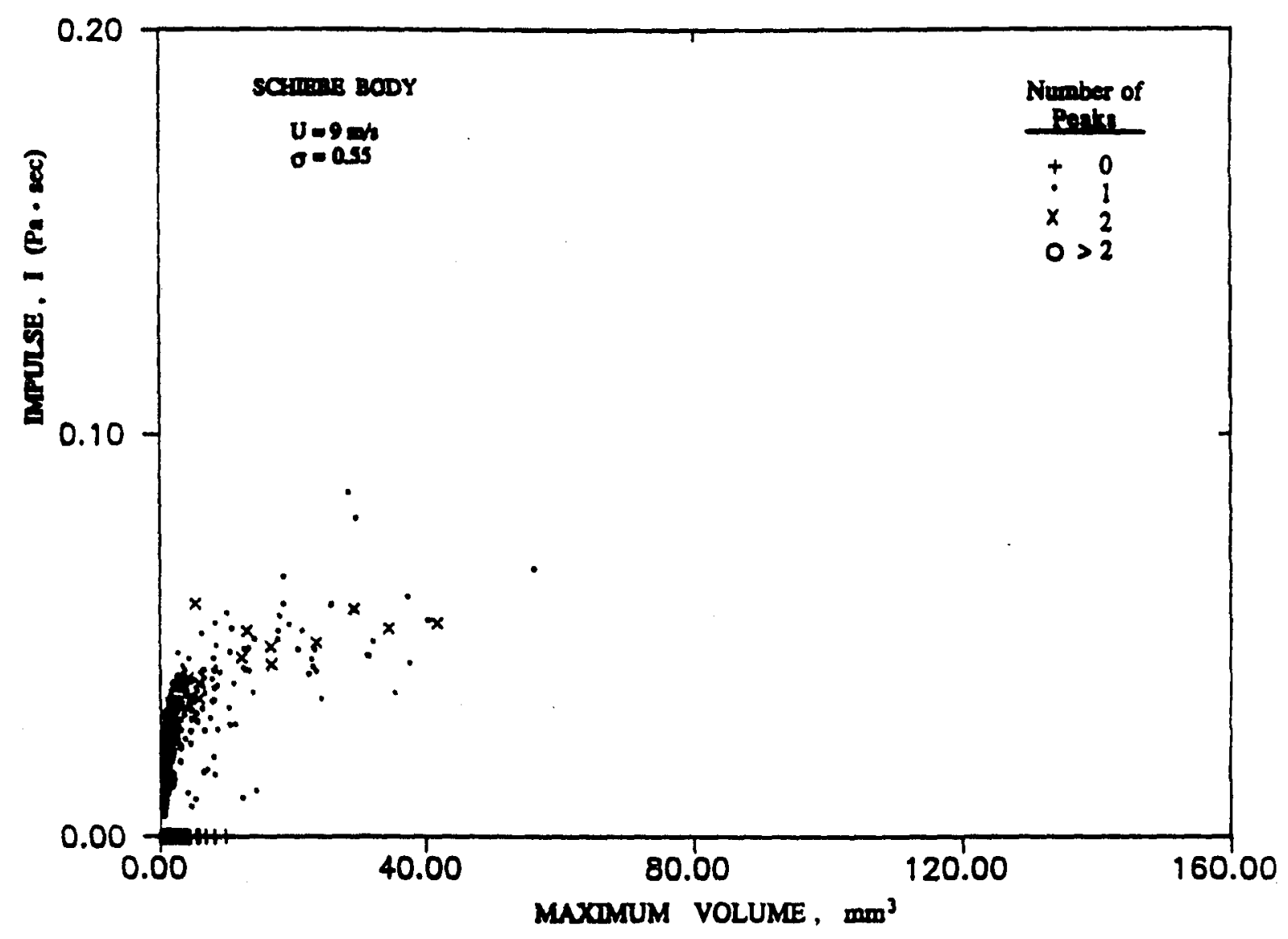

Figure 6.6 Impulse v. maximum bubble volume for Schiebe body, $U=9 \mathrm{~m} / \mathrm{s}$, $\sigma=0.55$. 


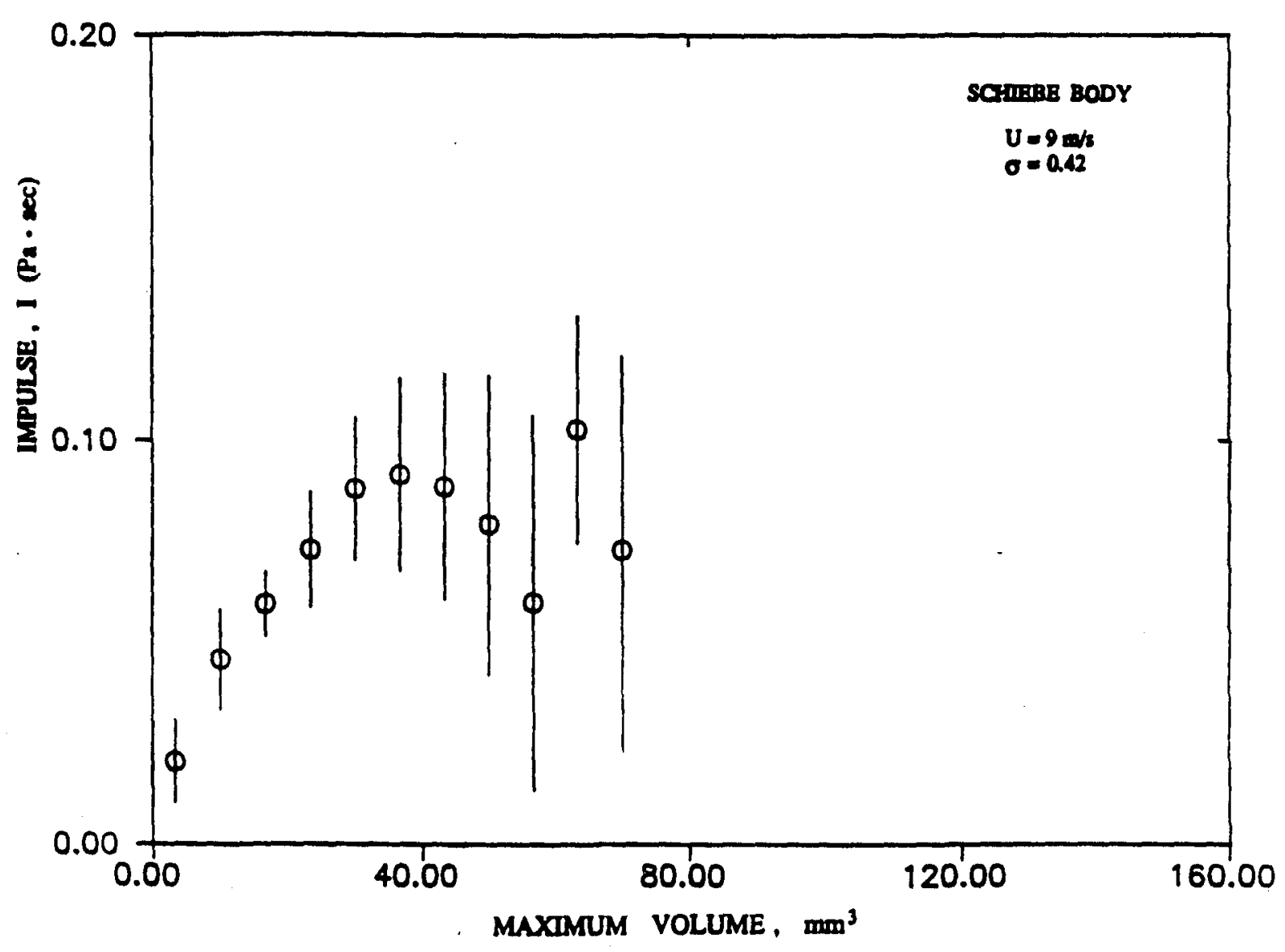

Figure 6.7 Averaged impuise v. maximum bubble volume for Schiebe body, $U=9 \mathrm{~m} / \mathrm{s}, \sigma=0.42$. 


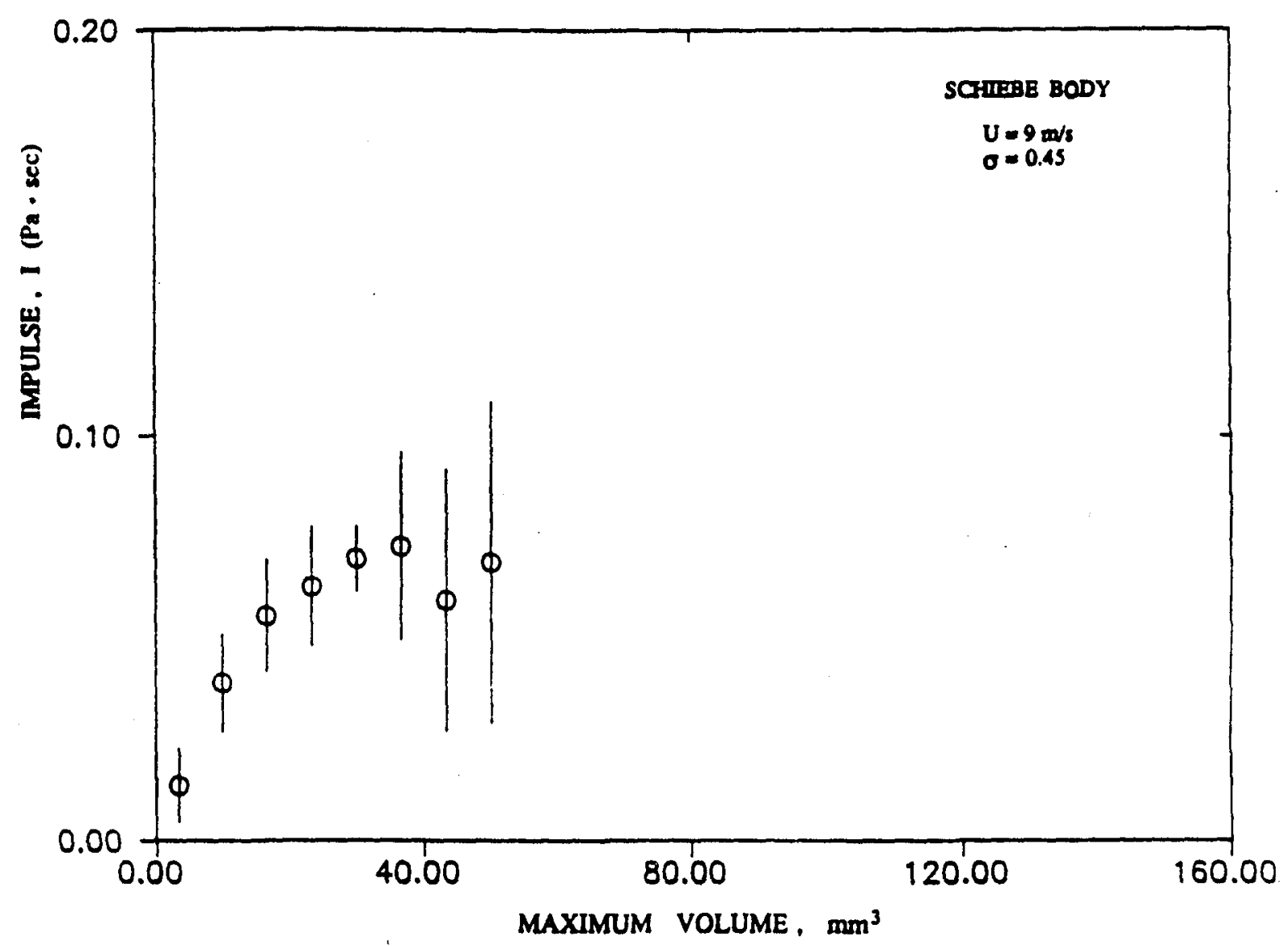

Figure 6.8 Averaged impulse v. maximum bubble volume for Schiebe body, $U=9 \mathrm{~m} / \mathrm{s}, \sigma=0.45$. 


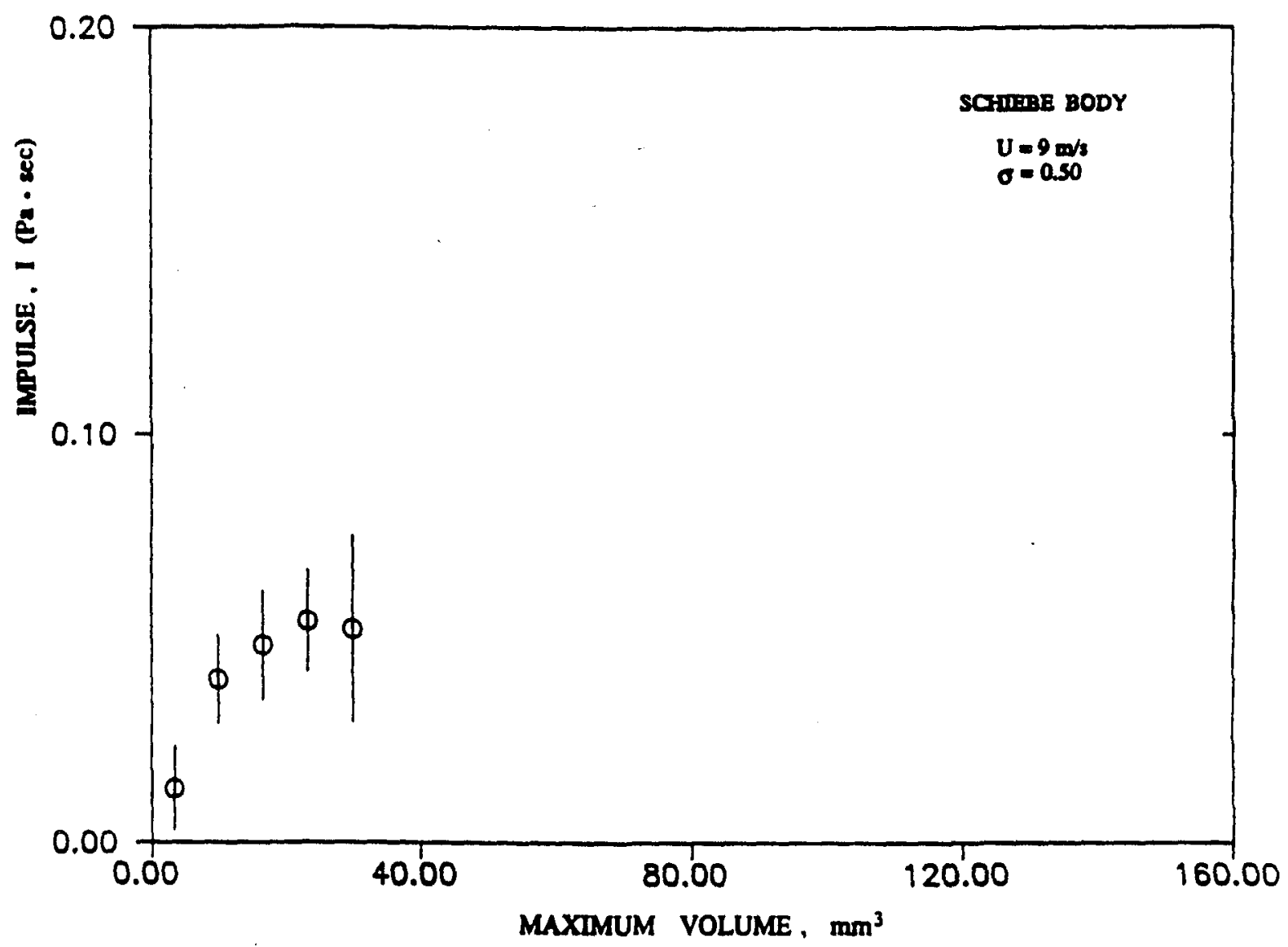

Figure 6.9 Averaged impulse v. maximum bubble volume for Schiebe body, $U=9 \mathrm{~m} / \mathrm{s}, \sigma=0.50$. 


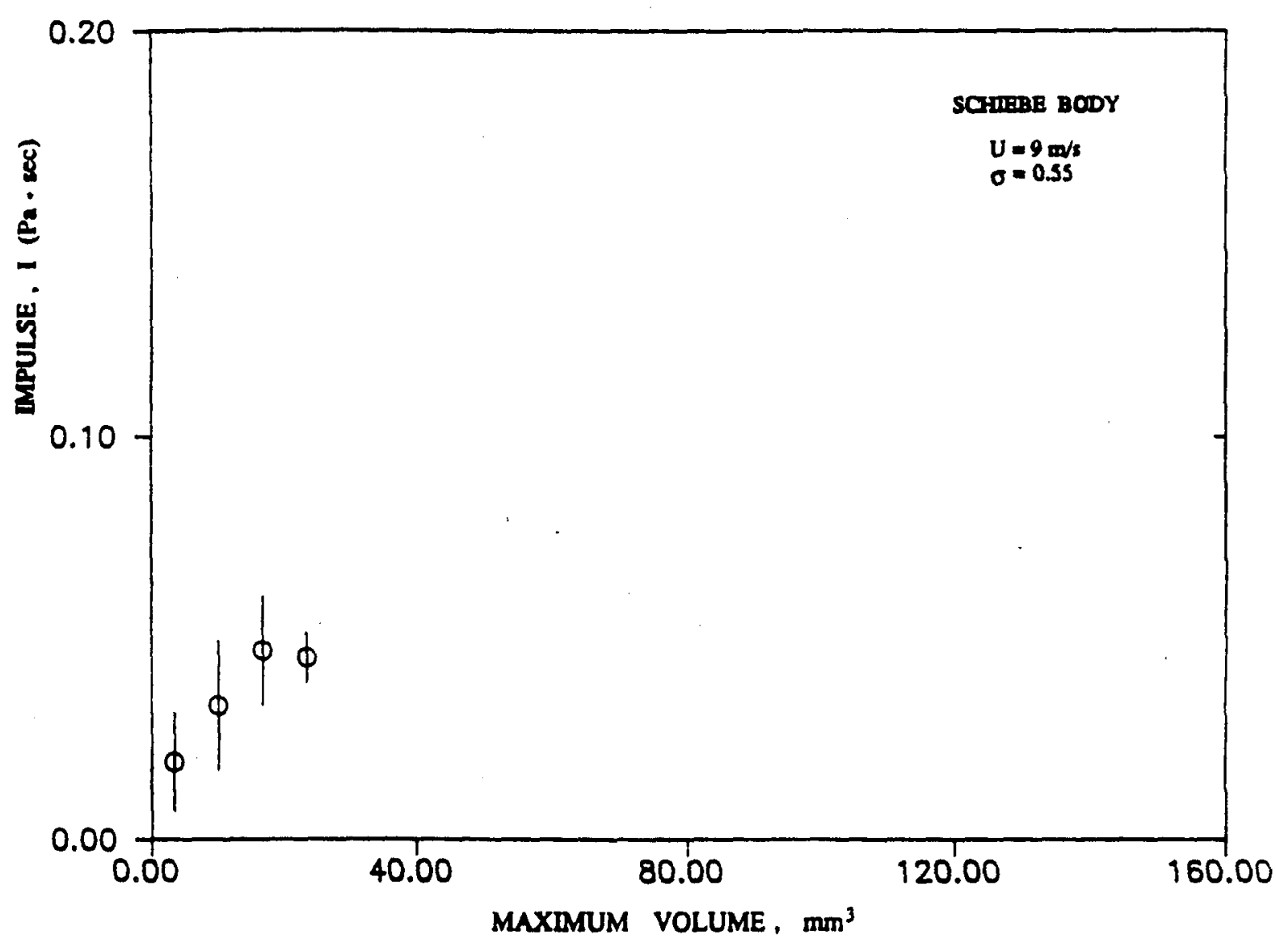

Figure 6.10 Averaged impulse v. maximum bubble volume for Schiebe body, $U=9 \mathrm{~m} / \mathrm{s}, \sigma=0.55$. 


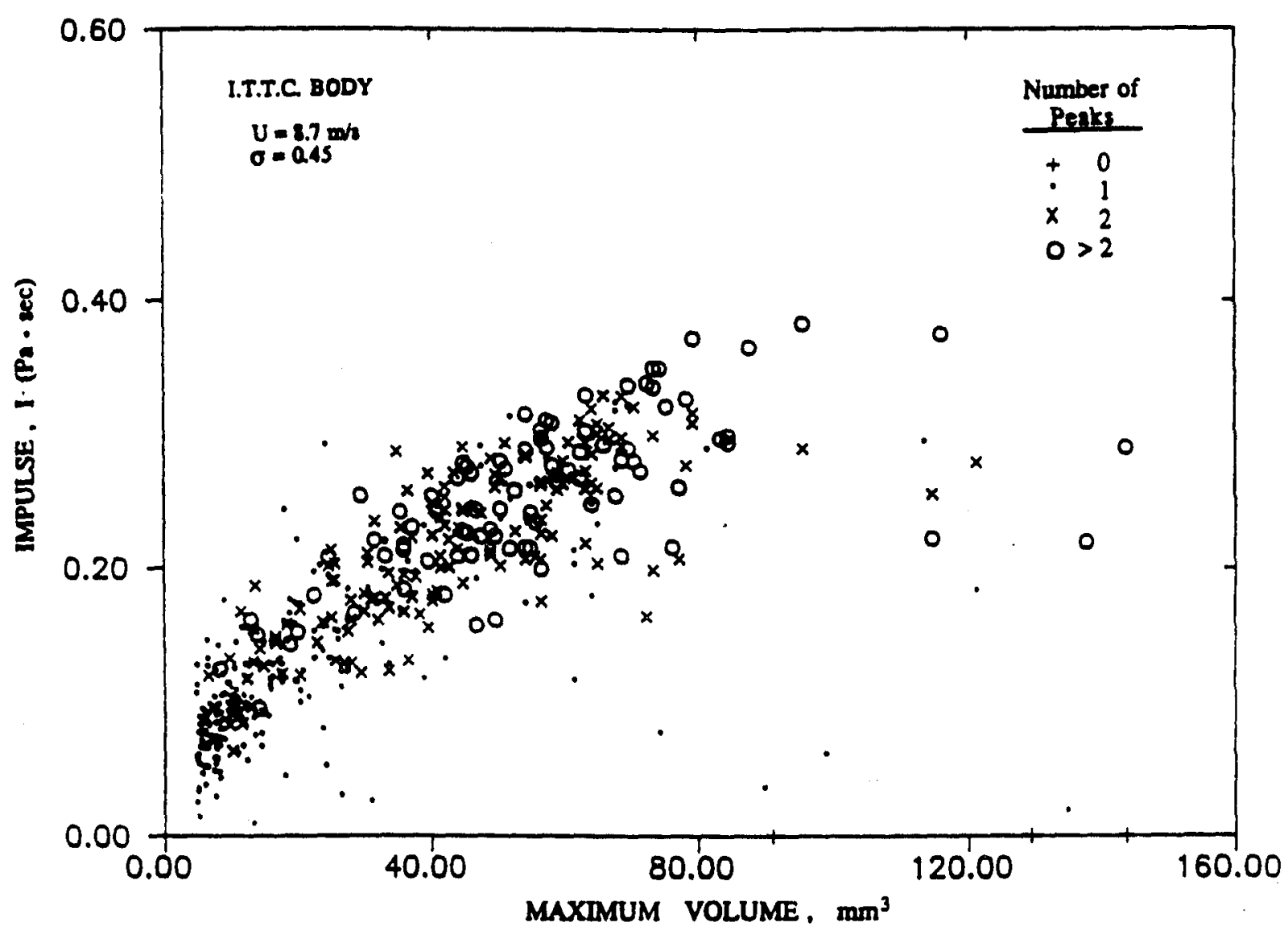

Figure 6.11 Impulse v. maximum bubble volume for I.T.T.C. body, $U=8.7 \mathrm{~m} / \mathrm{s}$, $\sigma=0.45$. 


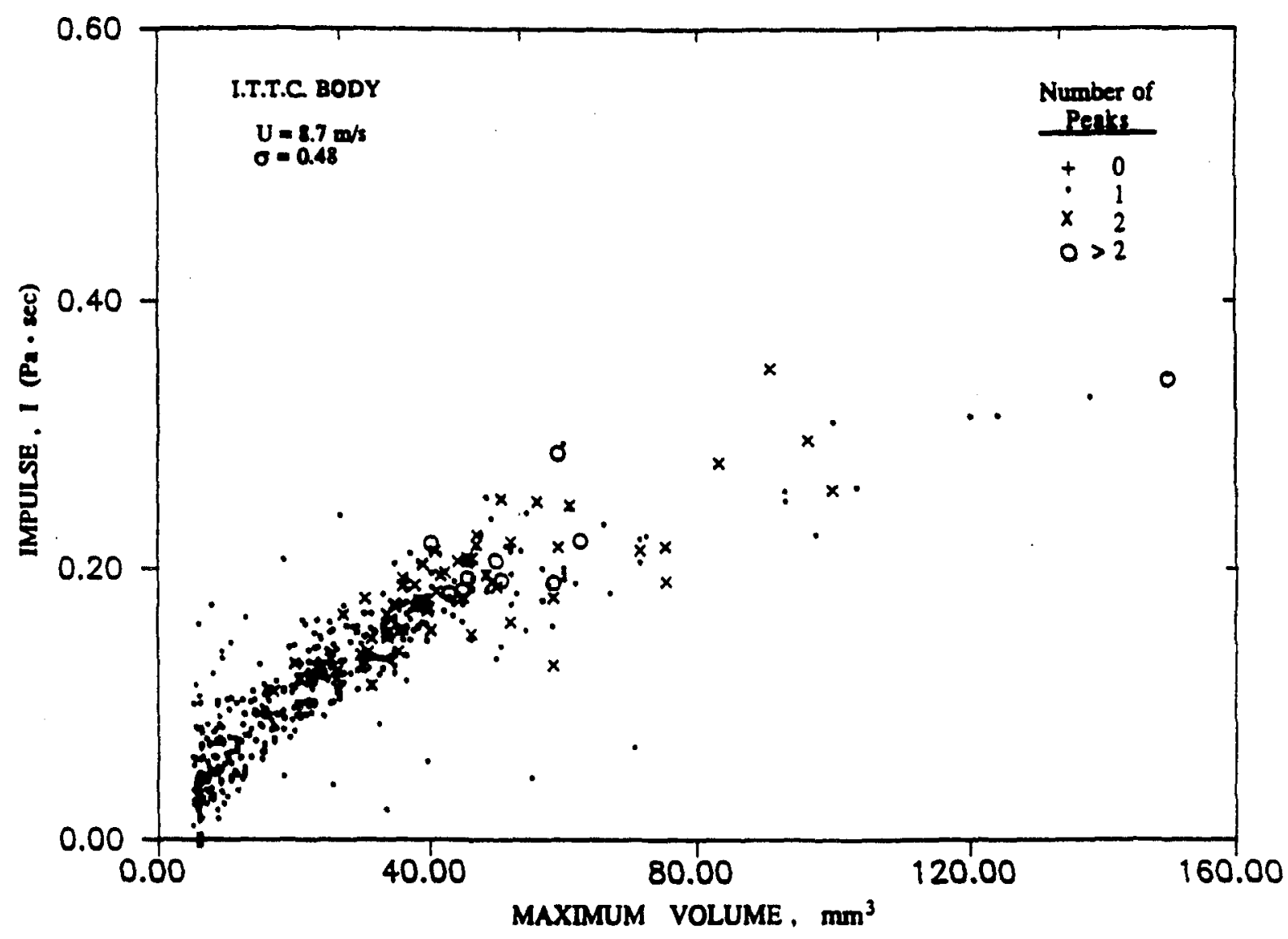

Figure 6.12 Impuise v. maximum bubble volume for I.T.T.C. body, $U=8.7 \mathrm{~m} / \mathrm{s}$, $\sigma=0.48$. 


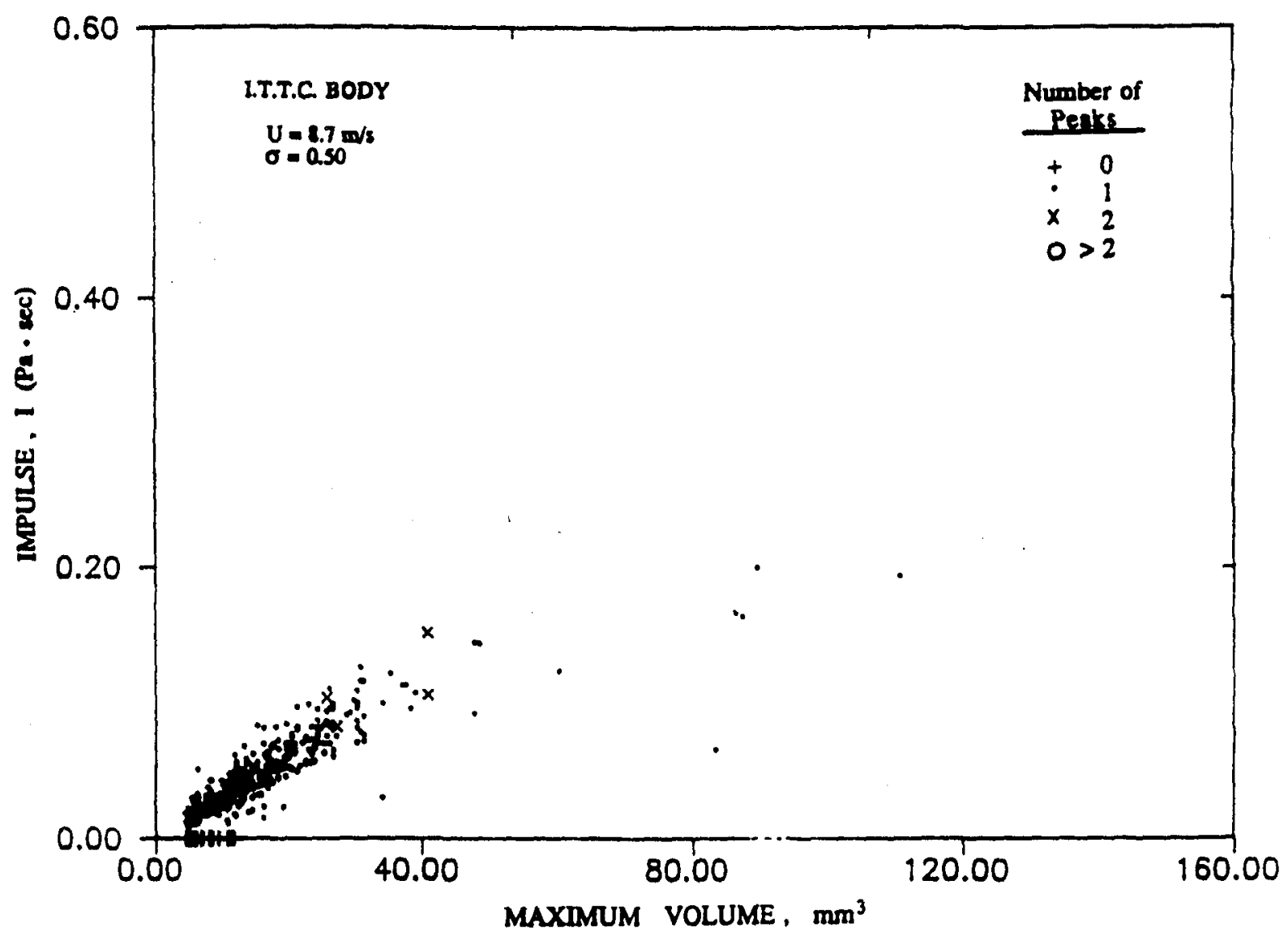

Figure 6.13 Impulse v. maximum bubble volume for I.T.T.C. body, $U=8.7 \mathrm{~m} / \mathrm{s}$, $\sigma=0.50$. 


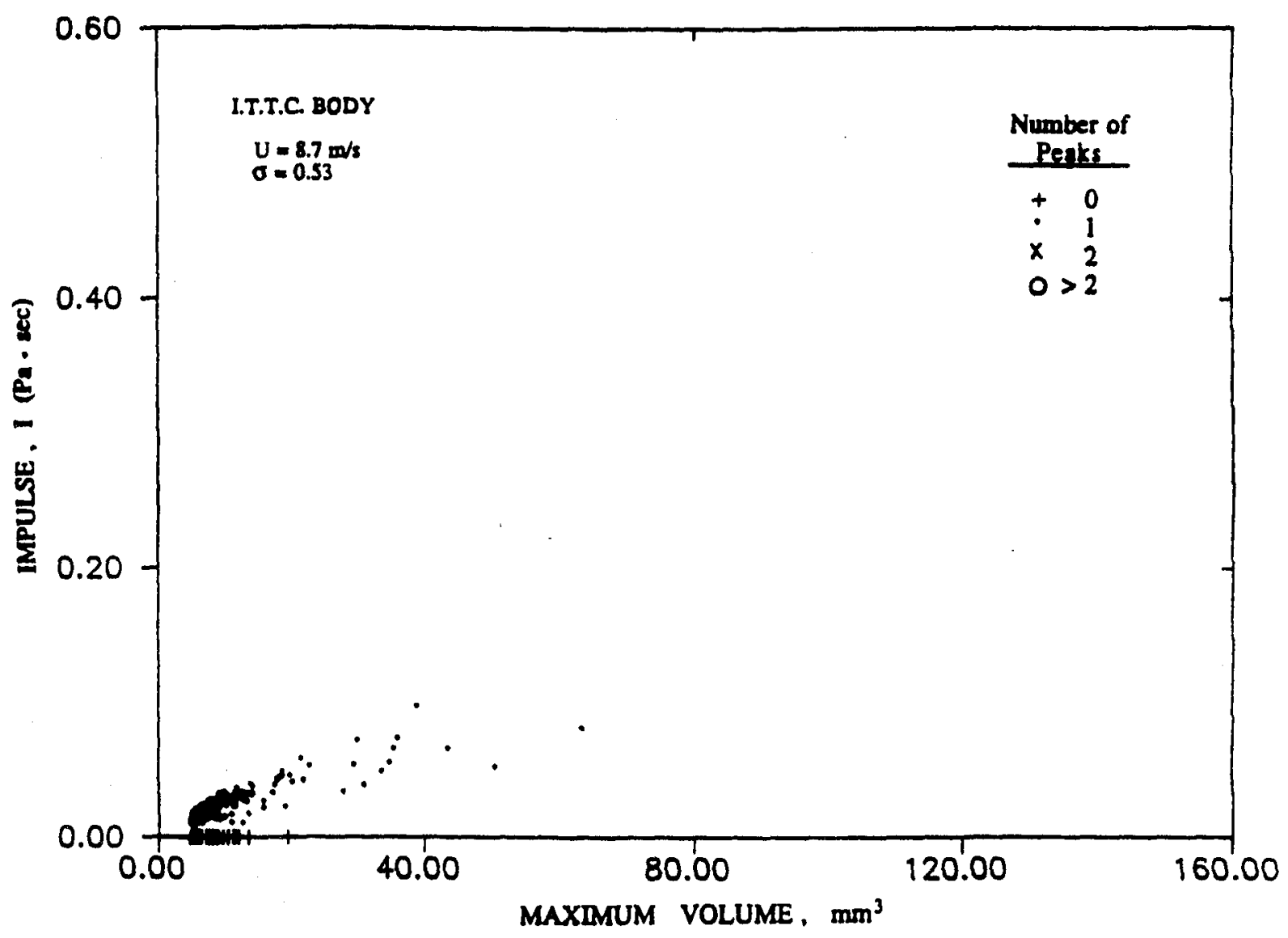

Figure 6.14 Impulse v. maximum bubble volume for I.T.T.C. body, $U=8.7 \mathrm{~m} / \mathrm{s}$, $\sigma=0.53$. 


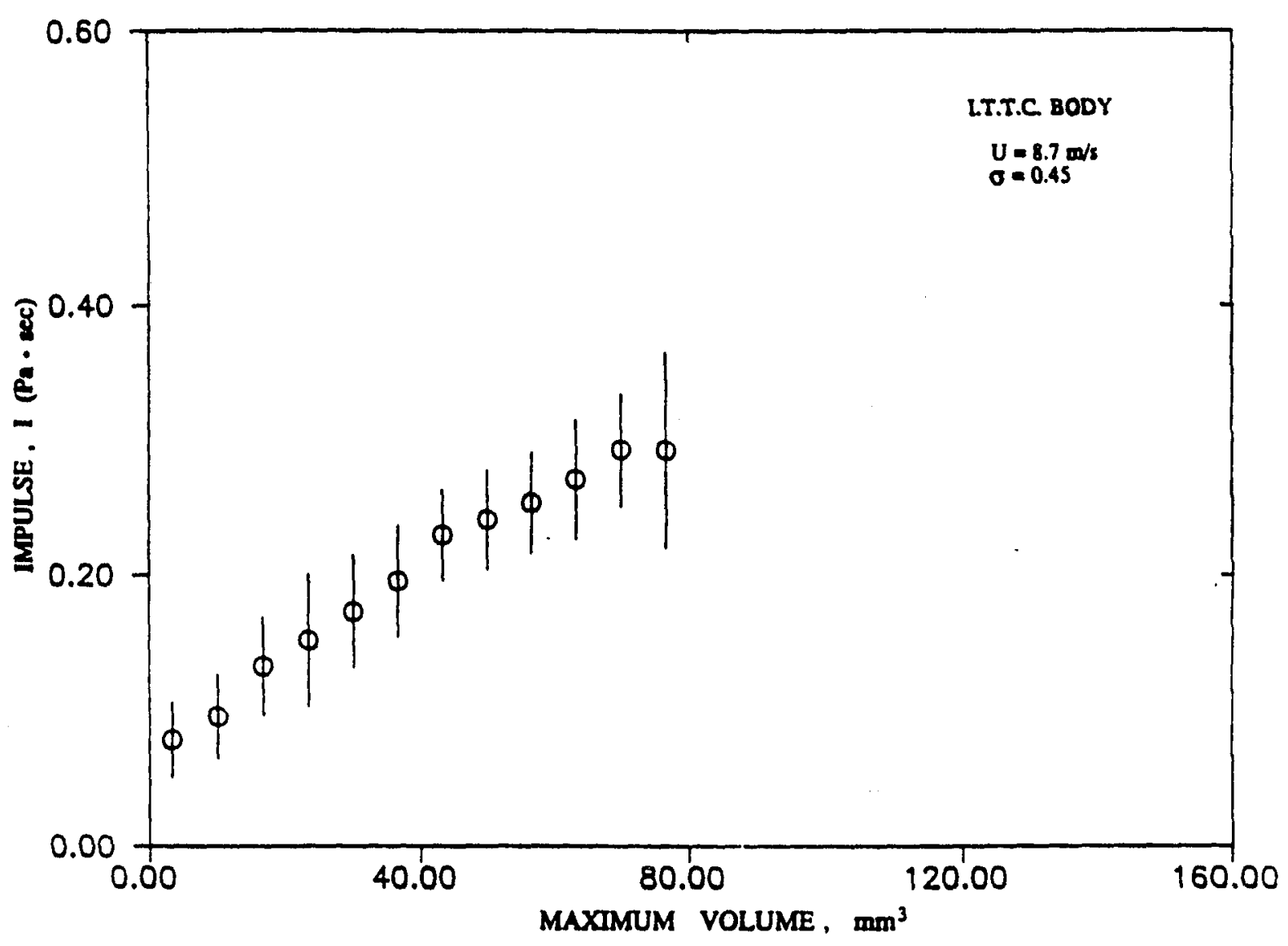

Figure 6.15 Averaged impulse v. maximum bubble volume for I.T.T.C. body, $U=8.7 \mathrm{~m} / \mathrm{s}, \sigma=0.45$. 


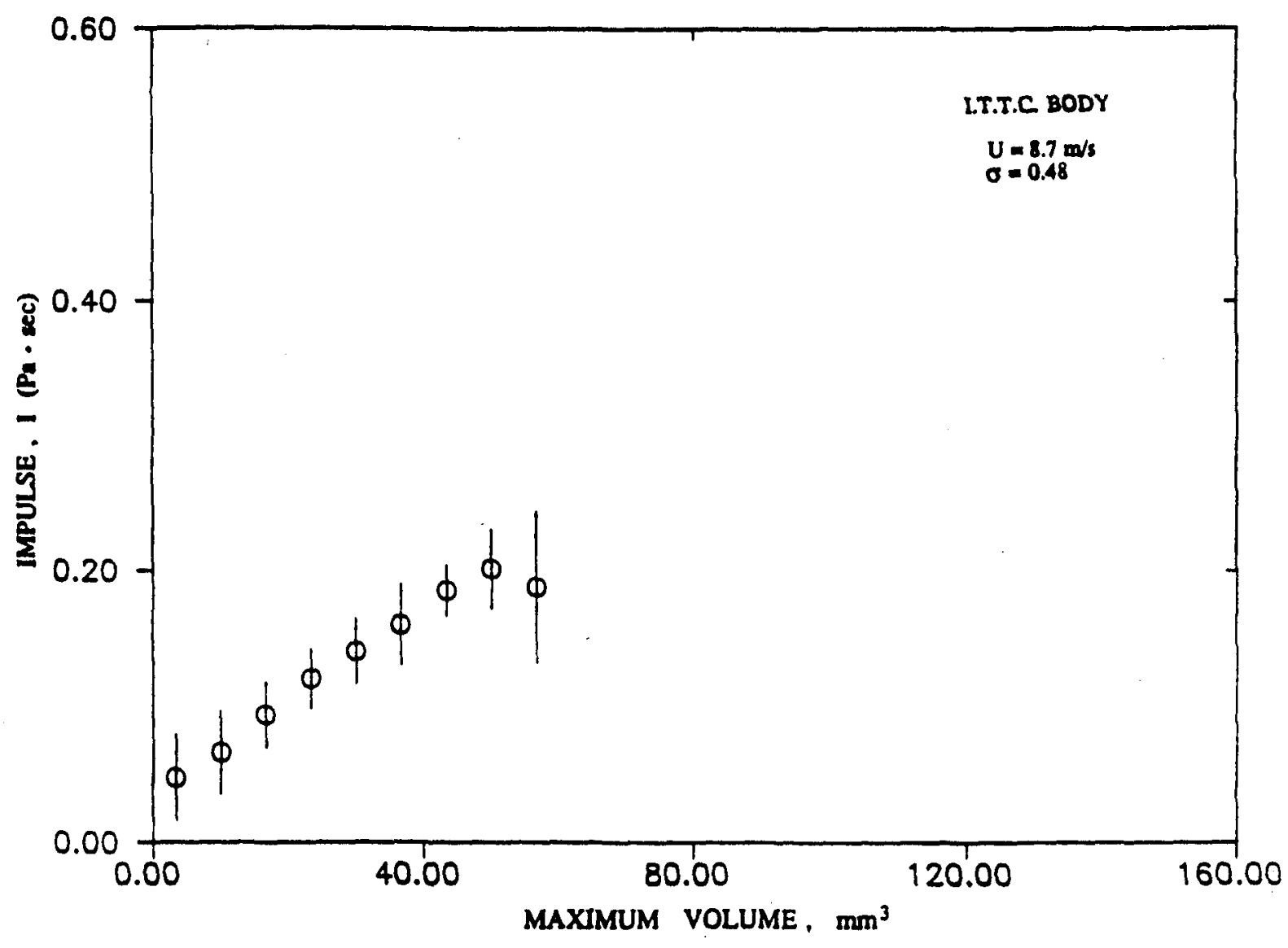

Figure 6.16 Averaged impulse v. maximum bubble volume for I.T.T.C. body, $U=8.7 \mathrm{~m} / \mathrm{s}, \sigma=0.48$. 


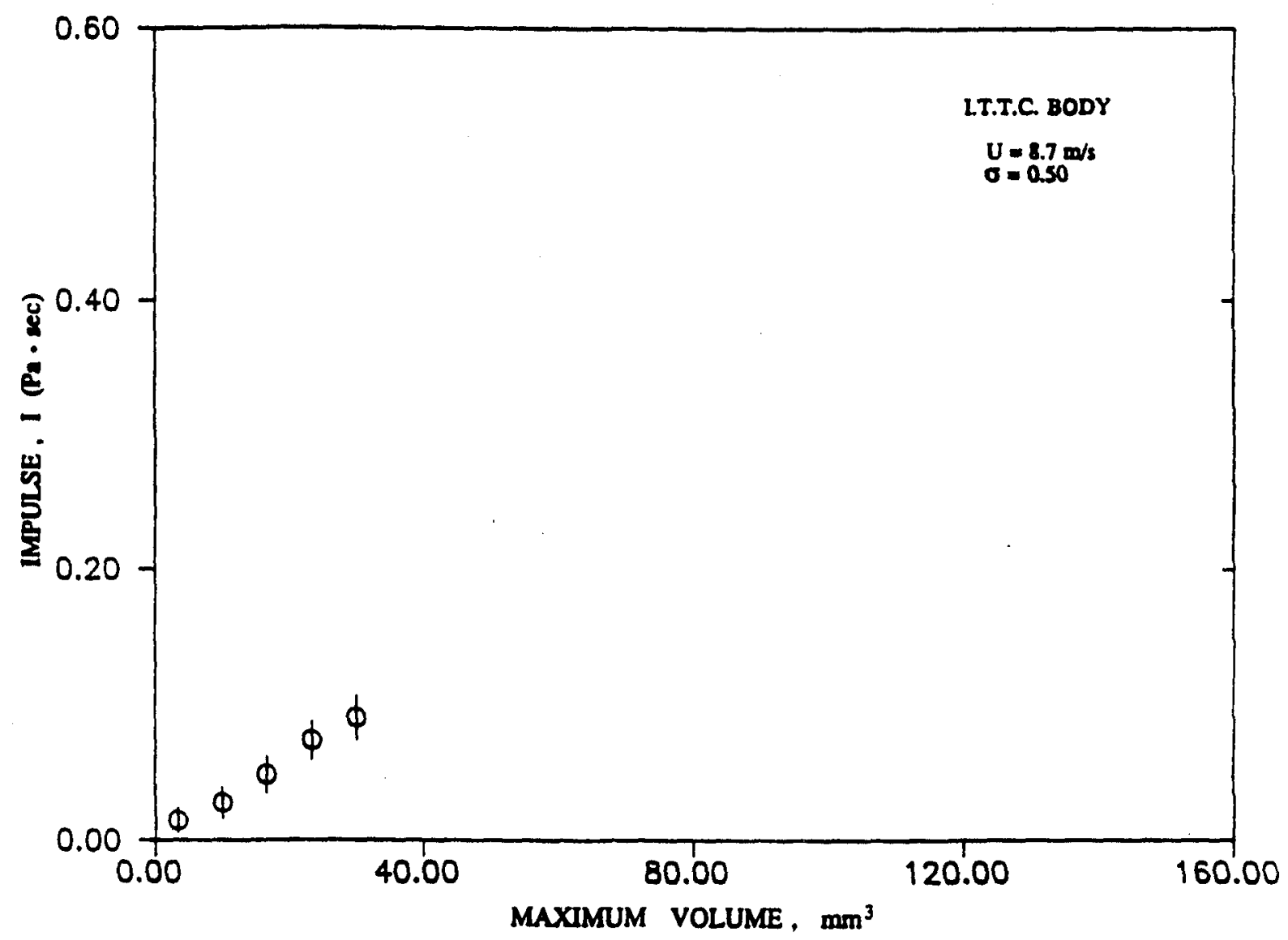

Figure 6.17 Averaged impulse v. maximum bubble volume for I.T.T.C. body, $U=8.7 \mathrm{~m} / \mathrm{s}, \sigma=0.50$. 


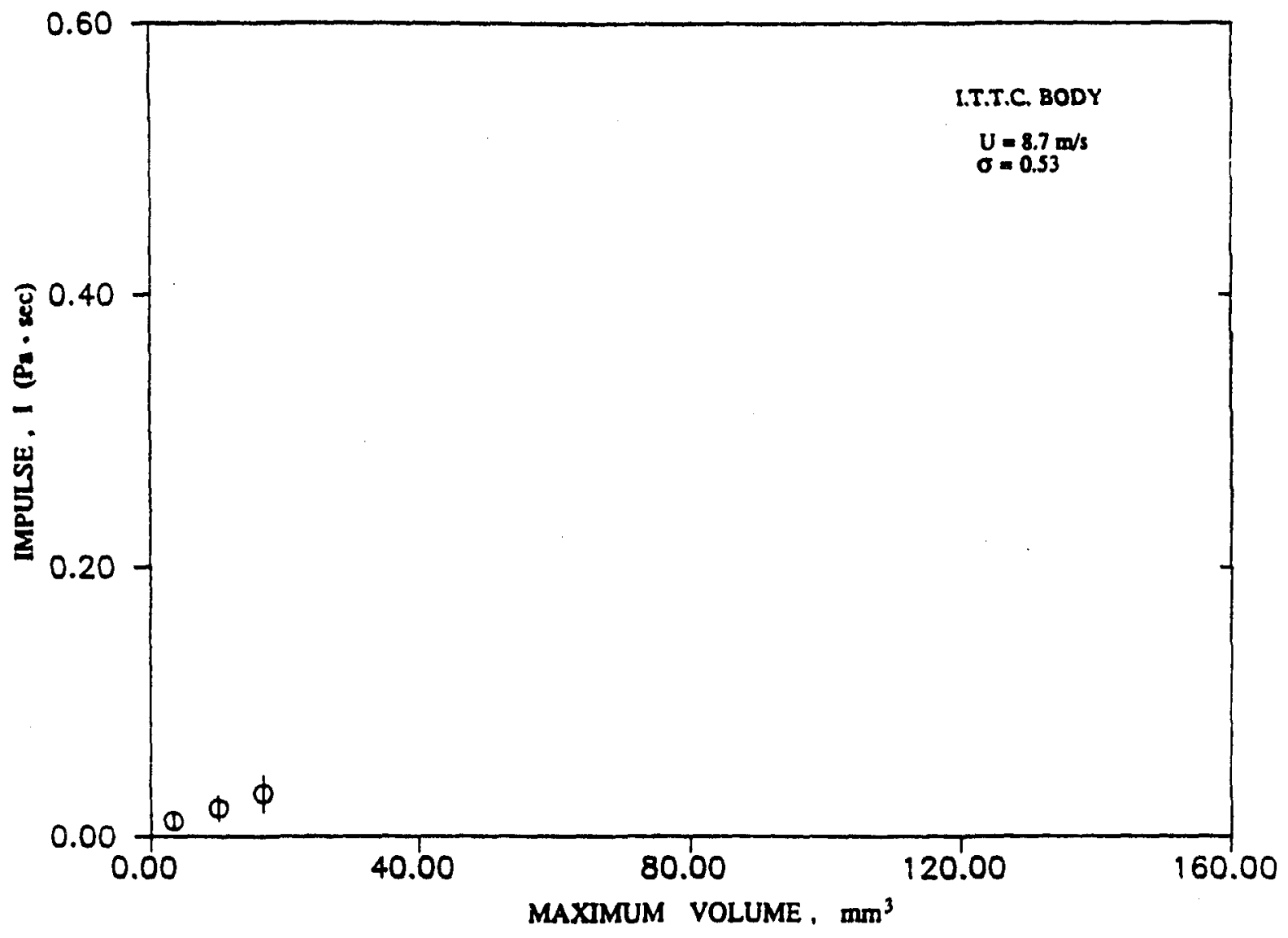

Figure 6.18 Averaged impuise v. maximum bubble volume for I.T.T.C. body, $U=8.7 \mathrm{~m} / \mathrm{s}, \sigma=0.53$. 


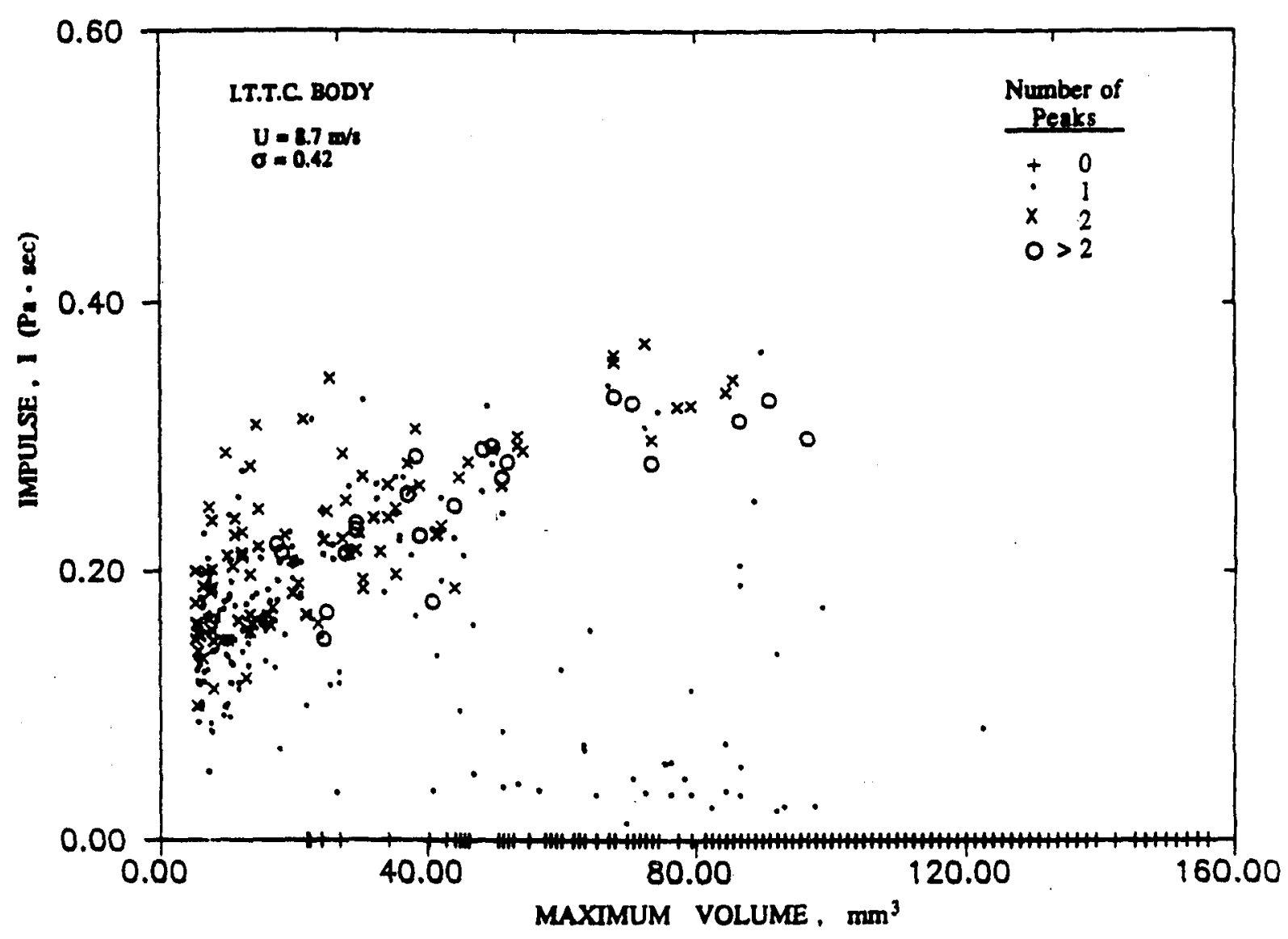

Figure 6.19 Impuise v. maximum bubble volume for I.T.T.C. body, $U=8.7 \mathrm{~m} / \mathrm{s}$, $\sigma=0.42$. 


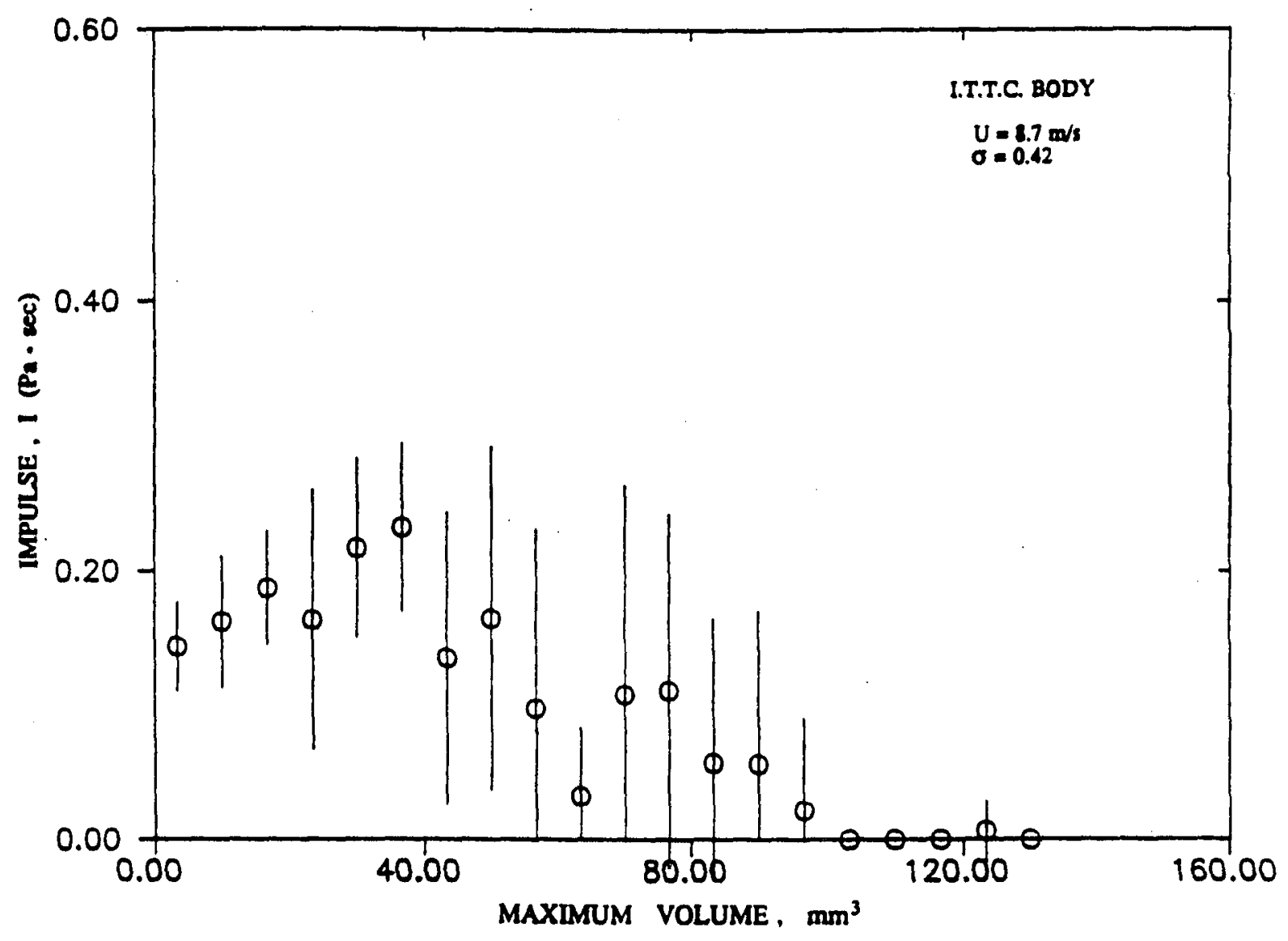

Figure 6.20 Averaged impulse v. maximum bubble volume for I.T.T.C. body, $U=8.7 \mathrm{~m} / \mathrm{s}, \sigma=0.42$. 


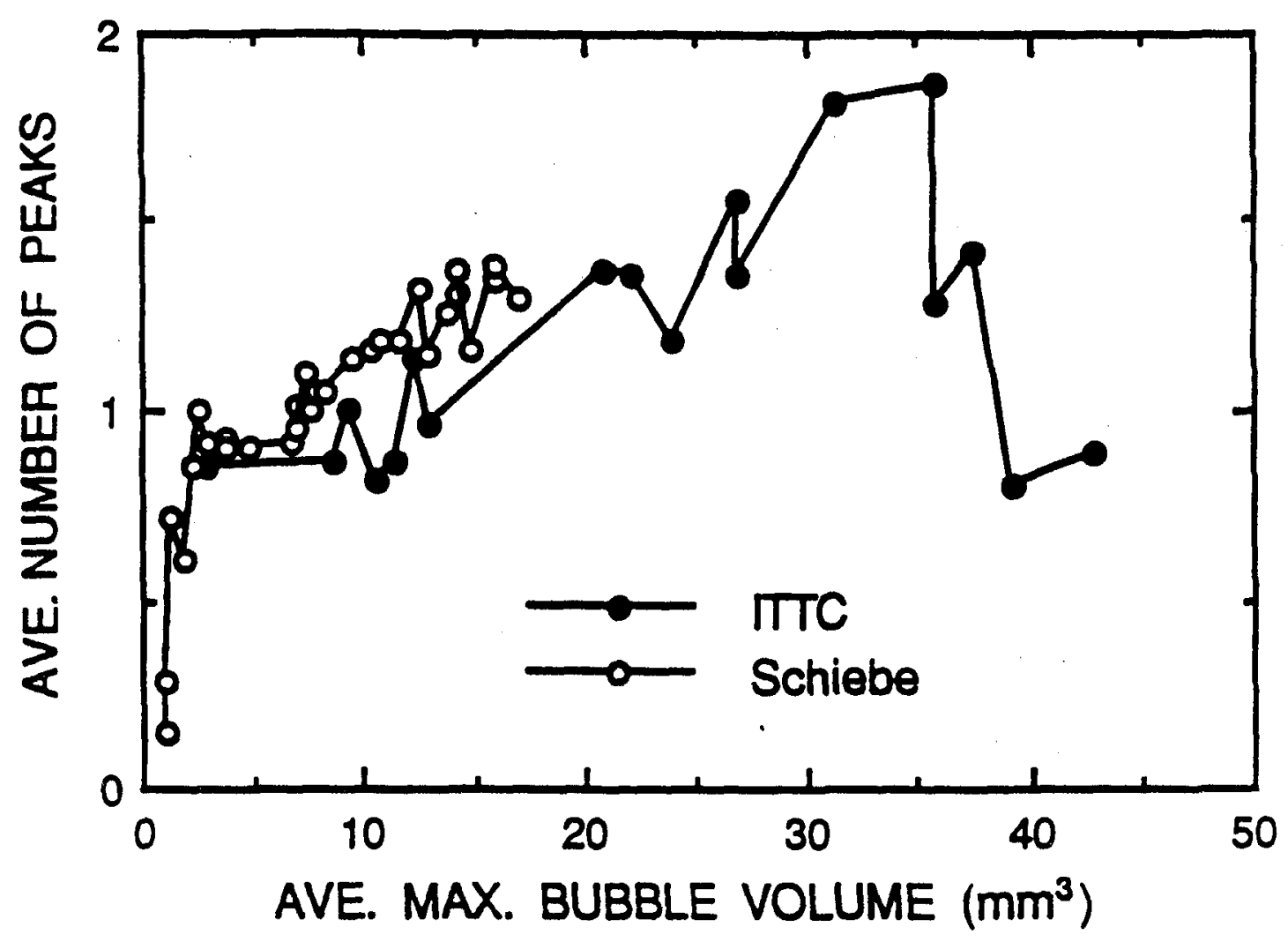

Figure 6.21 Average number of peaks v. ayerage maximum bubble volume for bubbles generated on the Schiebe body and I.T.T.C. body. 


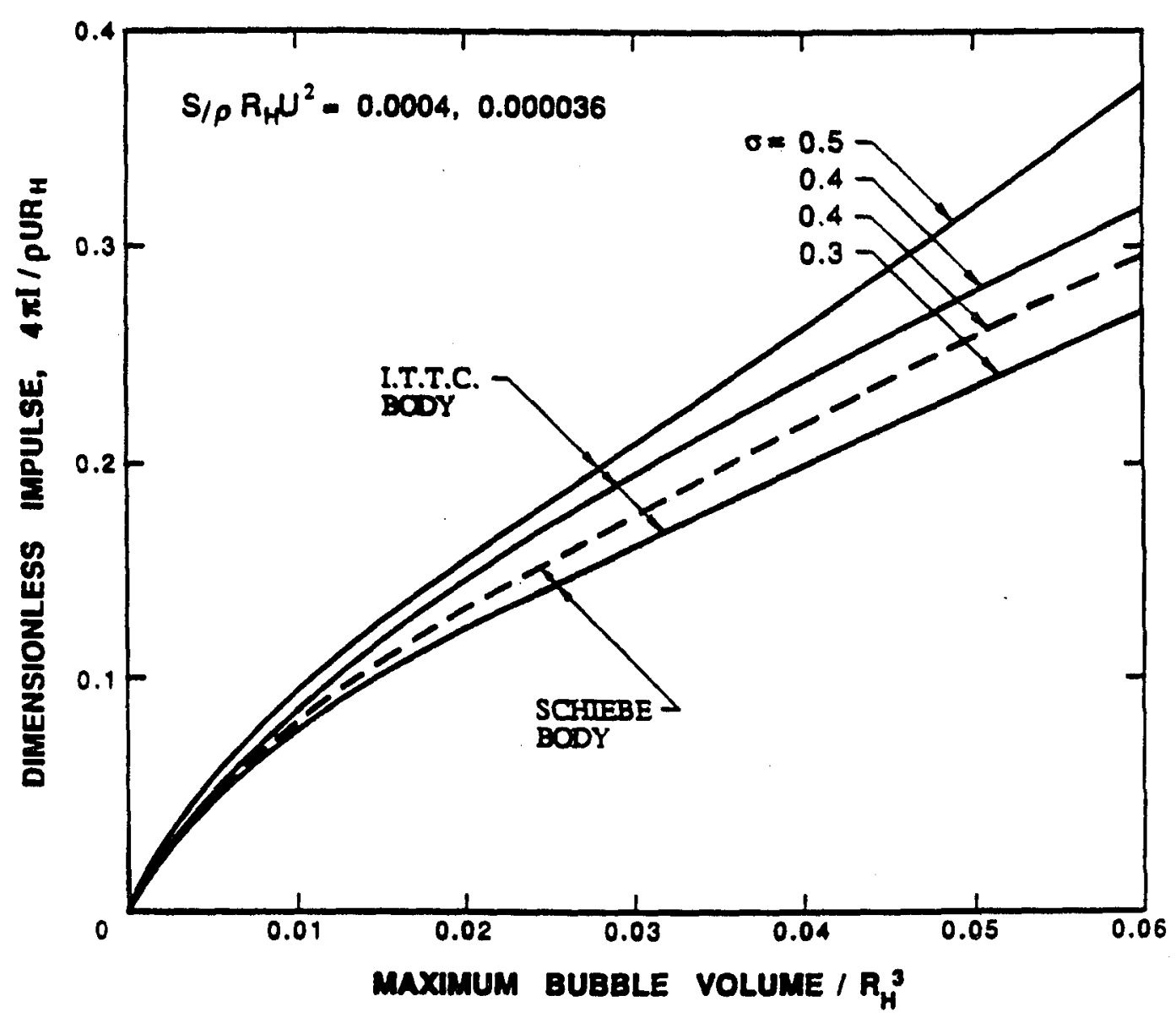

Figure 6.22 Numerical calculation of impulse v. maximum bubble volume for bubbles generated on the Schiebe body and I.T.T.C. body. 


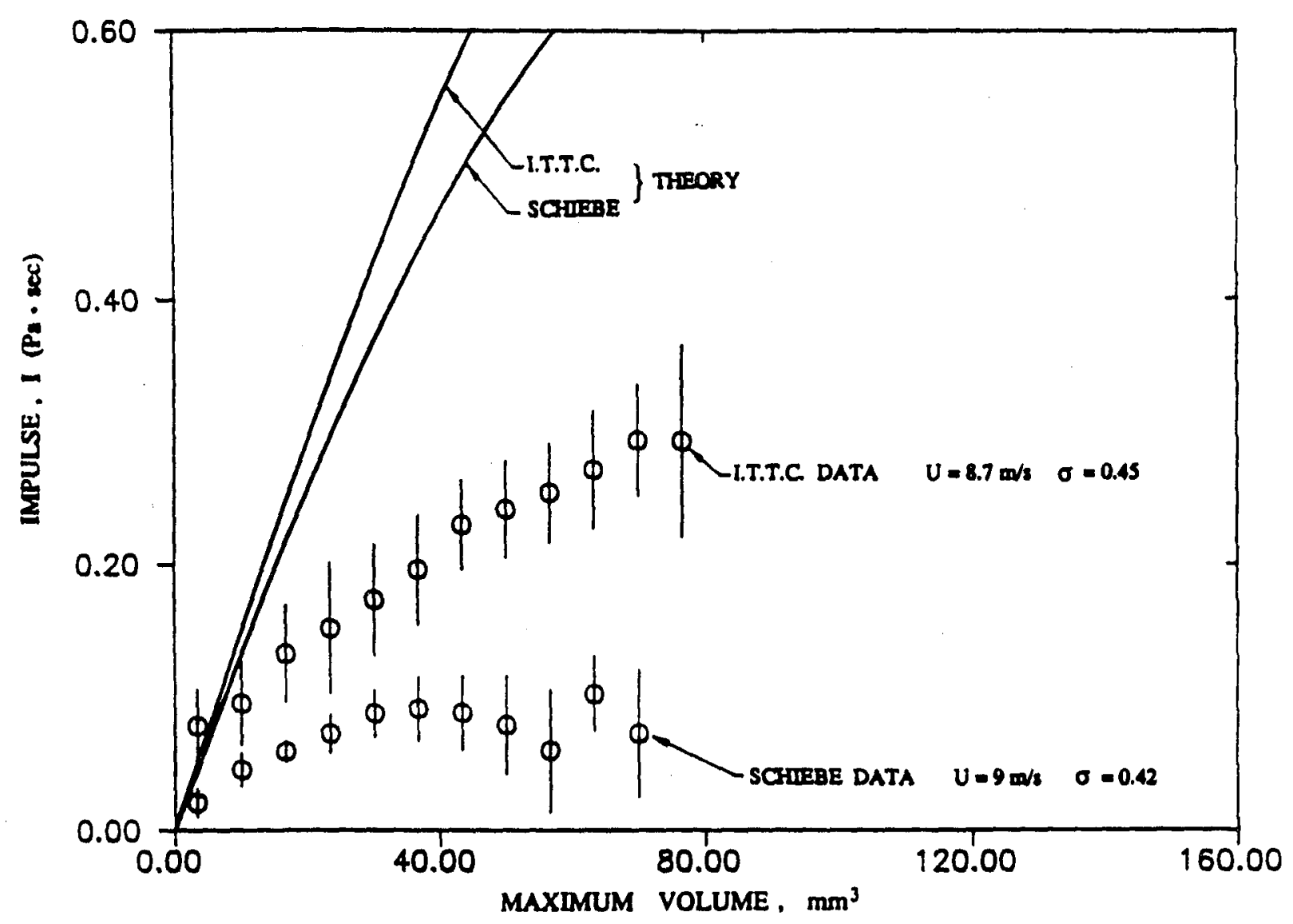

Figure 6.23 Comparison of theoretically predicted and experimentally measured impulse v. maximum bubble volume relationship for bubbles generated on the Schiebe body and I.T.T.C. body. Experimental data for $\sigma=0.45, U=9 \mathrm{~m} / \mathrm{s}$ for the Sciebe body and $U=8.7 \mathrm{~m} / \mathrm{s}$ for the I.T.T.C. body. 


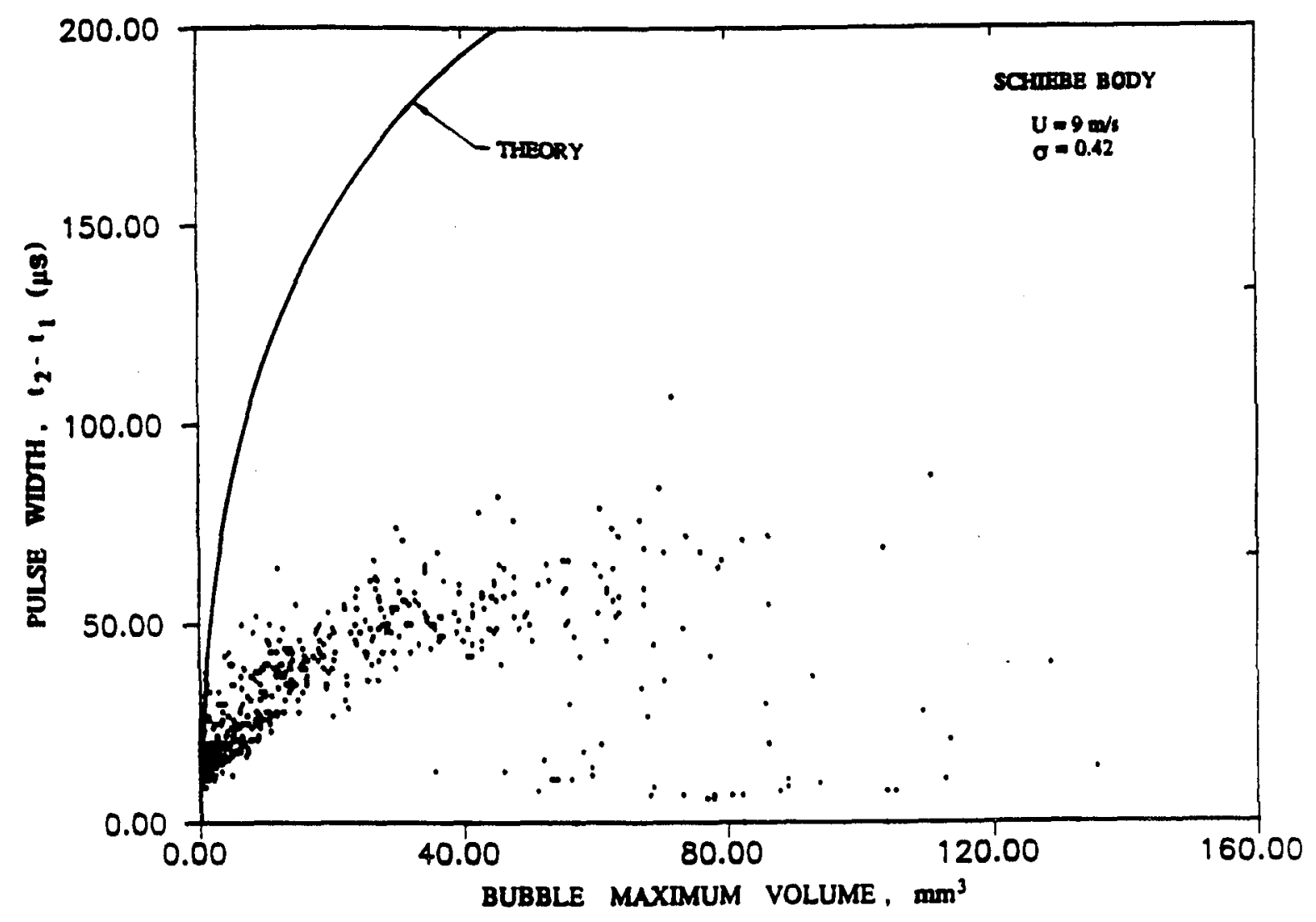

Figure 6.24 Comparison of theoretically predicted and experimentally measured pulse width v. maximum bubble volume relationship for bubbles on the Schiebe body, $U=9 \mathrm{~m} / \mathrm{s}$ and $\sigma=0.45$. 


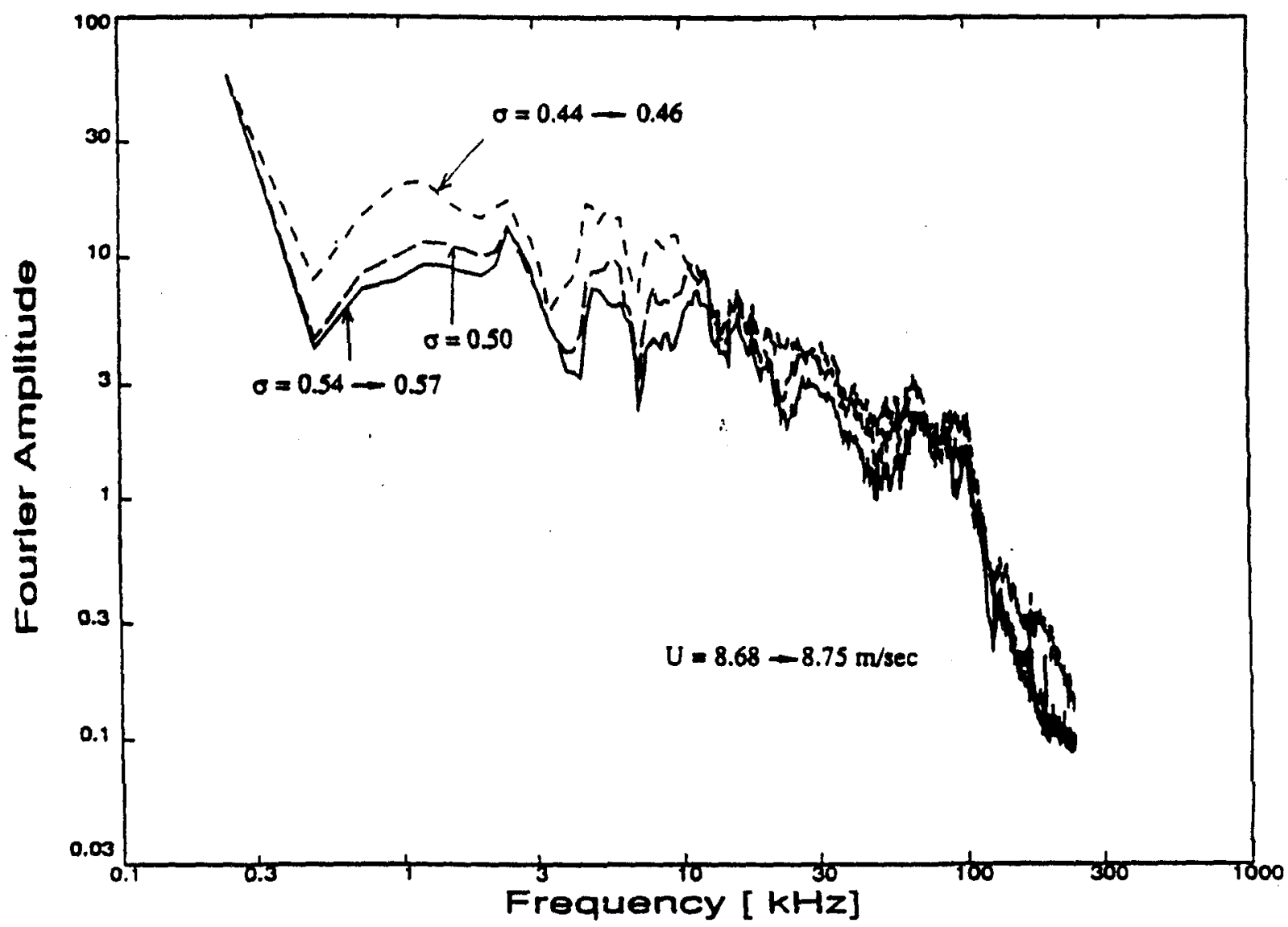

Figure 6.25 Averaged acoustic spectra derived from acoustic pulses generated by bubbles on the Schiebe body at average $U=8.7 \mathrm{~m} / \mathrm{s}$ and average $\sigma=0.45,0.50$, and $0.56 \mathrm{~m} / \mathrm{s}$. 


\section{CHAPTER 7 \\ 7. Observations of Cavitation Event Rate and Bubble Maximum Size Distributions}

\subsection{Introduction}

A complete understanding of a cavitating flow may not be gained solely from the behavior of individual bubbles. Bubble cavitation is the result of a specific flux of nuclei encountering a low pressure region, and a detailed knowledge of the nuclei population and cavitation event statistics is vital to the understanding of the cavitation process.

The stochastic nature of cavitation noise has been considered by several researchers (Akulichev and Ol'sheviskii (1968), Il'ichev (1968), and Lyamshev (1970)). Morozov (1969) showed that a cavitation process modeled after a Poisson process will produce a noise spectrum equivalent to the scaled spectrum of an average single cavitation event. More complicated statistical processes have been treated by Baiter (1986) where cavitation clustering has been analyzed. Cavitation noise scaling of the type introduced by Blake, Wolpert, and Geib (1977) will also be significantly influenced by the statistics of cavitating flows. As more nuclei become involved in cavitation, the intensity of the radiated noise will change.

The influence of nuclei flux on the cavitation event statistics of a given flow has been studied analytically and experimentally (Schiebe (1972), Baiter (1974) and Meyer, Billet, and Holl (1989)) yeilding a qualitative understanding of this relationship. The nuclei number distribution may be determined experimentally, but cavitation event statistics have only been deduced indirectly through the use of acoustic pulse counting. This technique may not yield accurate results considering some bubbles may produce more than one acoustic pulse while others may emit no noise at all.

The electrode probe can measure the occurrence of all cavitation events and 
readily produce event statistics for flows with limited cavitation. Experiments were performed to measure these statistics for cavitation on axisymmetric headforms, and the results are compared to simple analytical models.

\subsection{Experimental Measurements}

\subsubsection{Cavitation Event Rate and Statistics}

The circular electrode geometry was used on both headforms to detect all of the cavitation events for flows with event rates that were not so large as to cause overlap. Thus statistical information such as the mean event rate and the distribution of bubble maximum volumes could be constructed. For a free stream velocity of $9 \mathrm{~m} / \mathrm{s}$, cavitation event rates as high as 500 events/sec. could be measured before significant event overlap occurred. However, most experiments were conducted with much lower event rates.

The cavitation event rate and the time between cavitation events $\Delta T$ was measured for several thousand events for specific fixed operating conditions. The cavitation event rate was a highly variable parameter, even for a fixed freestream velocity and cavitation number. Figure 7.1 shows a typical event rate history for an experimental run. Each operating point was nominally held fixed for approximately 5 minutes. As cavitation bubbles are generated, the event rate increases even at a fixed operating point. This is due to the continual generation of nuclei by the collapsing bubbles and the change that occurrs when these nuclei complete their circuit in the water tunnel (as mentioned in Chapter 4). Since the LTWT does not. possess a resorber, the event rate is highly dependant on the tunnel history.

A typical example of the $\Delta T$ distribution is provided in Figure 7.2. Allowing for the averaging effect near the origin, the shape is approximated by a Poisson distribution, and this is expected for randomly distributed nuclei in the free stream. Consequently, the total cavitation noise spectra produced by these flows should be equivalent to the composite spectra presented in Figure 6.25. 


\subsubsection{Bubble Maximum Size Distribution}

Figure 7.3 shows examples of bubble maximum size distributions for cavitation on the Schiebe headform. Note that the bubble maximum sizes are presented as reduced radii. The reduced bubble radius is the radius of a sphere of volume equal to the measured bubble volume. Although the four bubble size distributions presented are all at the same cavitation number and tunnel velocity, their event rates and size distributions are quite different. Since the cavitation bubble maximum volume distribution is directly related to the incoming nuclei number distribution, these results clearly indicate that the nuclei number distribution can be quite different for the same tunnel operating conditions, even though for periods of up to 15 minutes the mean nuclei population would remain relatively constant. Weak control of the number of nuclei was affected through deaeration and nuclei injection. But, as Figure 7.3 indicates, the nuclei number distribution is a highly variable factor that influences travelling bubble cavitation and cavitation noise.

\subsubsection{Nuclei Number Distribution}

Using inline holography, the free stream nuclei population was measured at the same time as the cavitation event rate (and bubble maximum size distribution) during experiments with both headforms. The smallest nucleus that could be detected with certainty was approximately $20 \mu \mathrm{m}$ in diameter. While many holograms were taken, only a few could be reduced to determine the nuclei number distribution due to the excessive time required for a single hologram to be read. Furthermore, the number of nuclei sampled by each hologram will vary, and the number is small when compared to the number of events measured with the electrical probe. Therefore, the holographic data, while providing an adequate measure of the number of nuclei per unit volume, can provide only a very crude measure of the nuclei number distribution. 


\subsection{Theoretical Considerations}

\subsubsection{Cavitation Event Rate}

As indicated in the last section, whether a nucleus cavitates is strongly determined by the local minimum pressure it experiences. On the surface of the headform, this pressure is given by the minimum pressure coefficient. On streamlines above the body surface, the fluid pressure may still be low enough to cause a nucleus to cavitate provided that the minimum pressure it experiences is below the critical pressure:

$$
-C_{P T} \geq \sigma+\frac{8 S \beta}{3 \rho U^{2}} \frac{1}{R_{O}}
$$

where $R_{O}$ is the nucleus radius, and $C_{P T}$ is the minimum pressure experienced along a particular streamline.

An incoming streamtube may therefore be defined for a nucleus of specific size such that the nucleus will always encounter a pressure low enough to cause it to cavitate during its flow around the body. The fluid capture area of this streamtube will be a function of the nuclei radius, $R_{O}$, the free stream cavitation number, and the flow geometry. By assuming that the pressure gradient normal to the surface corresponds to the centrifugal pressure gradient caused by the radius of curvature, $\kappa$, of the surface at the minimum pressure point, and by assuming no slip between the nuclei and the fluid, the following expression for the nuclei capture area, $A\left(R_{O}\right)$, may be readily obtained:

$$
A\left(R_{O}\right)=\frac{-R_{B} \kappa \pi}{\sqrt{1-C_{P M}}}\left(\sigma+C_{P M}\right)\left(1-\frac{R_{C}}{R_{O}}\right)
$$

where $R_{O}$ is the original nuclei radius, $R_{B}$ is the headform radius at the point of minimum pressure, and $R_{C}$ is the minimum cavitatable nucleus given by Equation 
5.6. Equation 7.2 may be rewritten as

$$
A\left(R_{O}\right)=A_{V}\left(1-\frac{R_{C}}{R_{O}}\right)
$$

where $A_{V}$ is the capture area enclosing all streamlines that involve pressures less than vapor pressure; note that $A_{V}$ is a function only of the flow geometry and free stream conditions. Finally, the total flux of cavitatable nuclei or total cavitation event rate, $\Theta$, is

$$
\Theta=\int_{R_{C}}^{\infty} A\left(R_{O}\right) N\left(R_{O}\right) U d R_{O}
$$

where $N\left(R_{O}\right)$ is the free stream nuclei number distribution, $N\left(R_{O}\right)$, defined so that $N\left(R_{O}\right) d R_{O}$ is the number of nuclei of size between $R_{O}$ and $R_{O}+d R_{O}$. It is important to emphasize that both the upstream nuclei number distribution, $N\left(R_{O}\right)$, and the flow geometry as manifest in $A\left(R_{O}\right)$ effect the cavitation event rate.

\subsubsection{Bubble Maximum Size Distribution}

Now consider the distribution of bubble maximum sizes that this process will produce. This distribution is the result of different nuclei trajectories and sizes. Cavitating nuclei travelling on streamlines farther away from the headform will not grow to the same maximum volume as those travelling near the surface. Consequently, a flux of uniform nuclei, $R_{O}$, will yield a distribution of bubble sizes denoted by

$$
\operatorname{Pr}_{O}\left(R_{M}\right)=f\left(R_{M}, R_{O}\right)
$$

where $\operatorname{Pr}_{O}$ is a probability distribution for the maximum bubble size $R_{M}$. Because of the slight dependence of bubble maximum size upon nucleus size, $\operatorname{Pr}_{O}$ is a function of $R_{O}$.

A flux of nuclei represented by the nuclei number distribution, $N\left(R_{O}\right)$, will therefore produce a distribution of maximum bubble sizes, $\operatorname{Pr}$, given by

$$
\operatorname{Pr}\left(R_{O}\right)=\frac{1}{\Theta} \int_{R_{C}}^{\infty} \operatorname{Pr} O A\left(R_{O}\right) N\left(R_{O}\right) U d R_{O}
$$


Figure 7.4 presents a calculated maximum bubble size distribution for the I.T.T.C. headform given a nuclei flux characterized by $N\left(R_{O}\right)=10^{-6} R_{O}^{-3.5}$ where $N\left(R_{O}\right)$ is in $m^{-4}$ and $R_{O}$ is in $m$. The results presented in Figure 5.1 relating the nuclei size to the maximum bubble size were used in the calculation.

If no relationship existed between nuclei size and the maximum bubble size, $\operatorname{Pr}$ would be independent of the nuclei number distribution; changes in $N\left(R_{O}\right)$ would merely change the total event rate. The experimental data indicate, however, that the bubble maximum size distributions are strongly influenced by the nuclei number distribution. The varying event rates reported in Figure 7.2 indicate different nuclei populations, and each example is accompanied by a unique bubble size distribution. The small influence of nuclei size upon the maximum bubble size will ultimately have a significant influence upon the bubble maximum size distribution.

\subsection{Comparison of Predicted and Measured Data}

In this section measurements of cavitation event rates and bubble maximum size distributions will be compared to the holographically-determined free stream nuclei number distribution. Figure 7.5a presents a measured nuclei number distribution with an approximate power law correlation for freestream conditions of $U=9 \mathrm{~m} / \mathrm{s}$ and $\sigma=0.45$, and Figure $7.5 \mathrm{~b}$ presents the resulting cavitation event rate and bubble maximum size distribution for cavitation on the I.T.T.C. body. The relations for the cavitation event rate and bubble maximum size distribution derived above were used to estimate these quantities using the measured nuclei number distribution of Figure 7.5a, and the results are shown in Figure 7.6.

The predicted cavitation event rate of 128 events/sec. is remarkably close to the measured event rate of 156 events/sec., although changes in the approximate analytical expression for the free stream nuclei number distribution would change the calculated event rate by about 50events/sec. Examination of other data sets also reveals a reasonably close correlation between the predicted and measured event 
rates, and these results will be presented in a later publication.

The close match between the predicted and measured event rates indicates that the nucleus stability criteria used adequately models the actual cavitation process. The minimum cavitatable nucleus for this flow calculated from Equation 5.6 is approximately $20 \mu \mathrm{m}$, and the measured nuclei number distribution indicate that most of the cavitating nuclei are in the range 20 to $100 \mu \mathrm{m}$. The success of the model suggests that the quantities $A_{V}$ and $R_{C}$ may be used to adequately characterize the nuclei capture area for flows over more complicated bodies.

The calculated bubble maximum volume distribution, however, departs substantially from the measured size distribution in terms of its details. The predicted bubble size range is about twice the observed size range, and the number of larger bubbles predicted is much smaller than the observed percentage. These discrepancies may be the result of several phenomena. First, the maximum size achieved by a nucleus subjected to a specific pressure history may not be adequately predicted by the Rayleigh-Plesset equation since bubble growth may be limited by the positive pressure gradients above the headform surface. Once the bubble has grown sufficiently, the mean pressure on the bubble surface will be larger than the surface pressure used in the Rayleigh-Plesset calculations, reducing the driving force for bubble growth. Furthermore, Johnson and Hsieh (1966) have suggested a "screening effect" in which larger nuclei would be forced away from the body surface, preventing them from cavitating. However, this effect may not be relevant for the range of nuclei sizes considered here, and Meyer, Billet, and Holl (1989) failed to note this effect in a calculation similar to those presented here.

Furthermore, the experimental bubble maximum size distributions often show several maxima that were repeatable for nominally fixed operating conditions, and these distributions cannot be simulated with simple, smooth functions representing the nuclei distribution and nucleus/maximum size relationship. It seems likely that these maxima are the result of a complex nuclei number distribution. While many 
holograms must be read to reveal such detail, the electrode probe easily measured thousands of events, making such detailed resolution possible for the first time.

\subsection{Conclusions}

The nuclei population of a recirculating water tunnel is a constantly varying parameter, and the relationship between the free stream nuclei flux and the resulting cavitation event rate and bubble maximum size distribution is complex. Yet, the cavitation event rate may be predicted using the simple models described above. This result suggests that the tendency of a body to cavitate may be estimated by simple parameters, such as $C_{P M}, A_{V}$, and $R_{C}$, derived from analysis of the non-cavitating flow around the body. Furthermore, by formulating the relationship between the cavitation event rate and the nuclei number distribution, cavitation noise generation and noise scaling may be better understood.

On the other hand, the maximum bubble size distribution is not adequately predicted by the models presented. This distribution is influenced by many factors, some of which are not fully characterized in the calculation, and the actual nuclei number distribution may contain detail that is difficult to measure holographically. Both these factors could explain the wide variation in observed bubble maximum size distributions. 


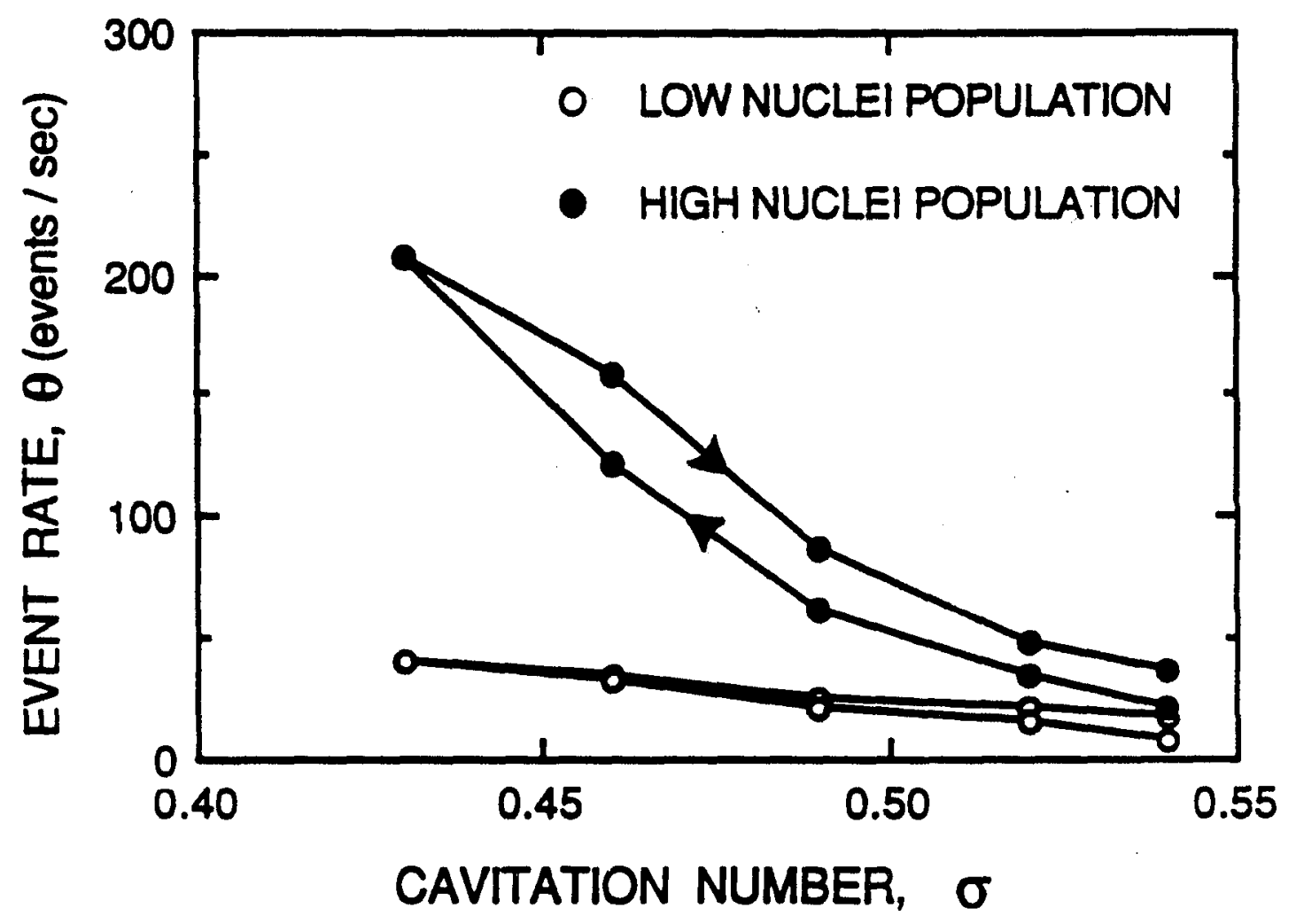

Figure 7.1 Cavitation event rate history for cavitation on the Schiebe body at $U=8.6 \mathrm{~m} / \mathrm{s}$. Operating points were held constant for 10 minutes. 


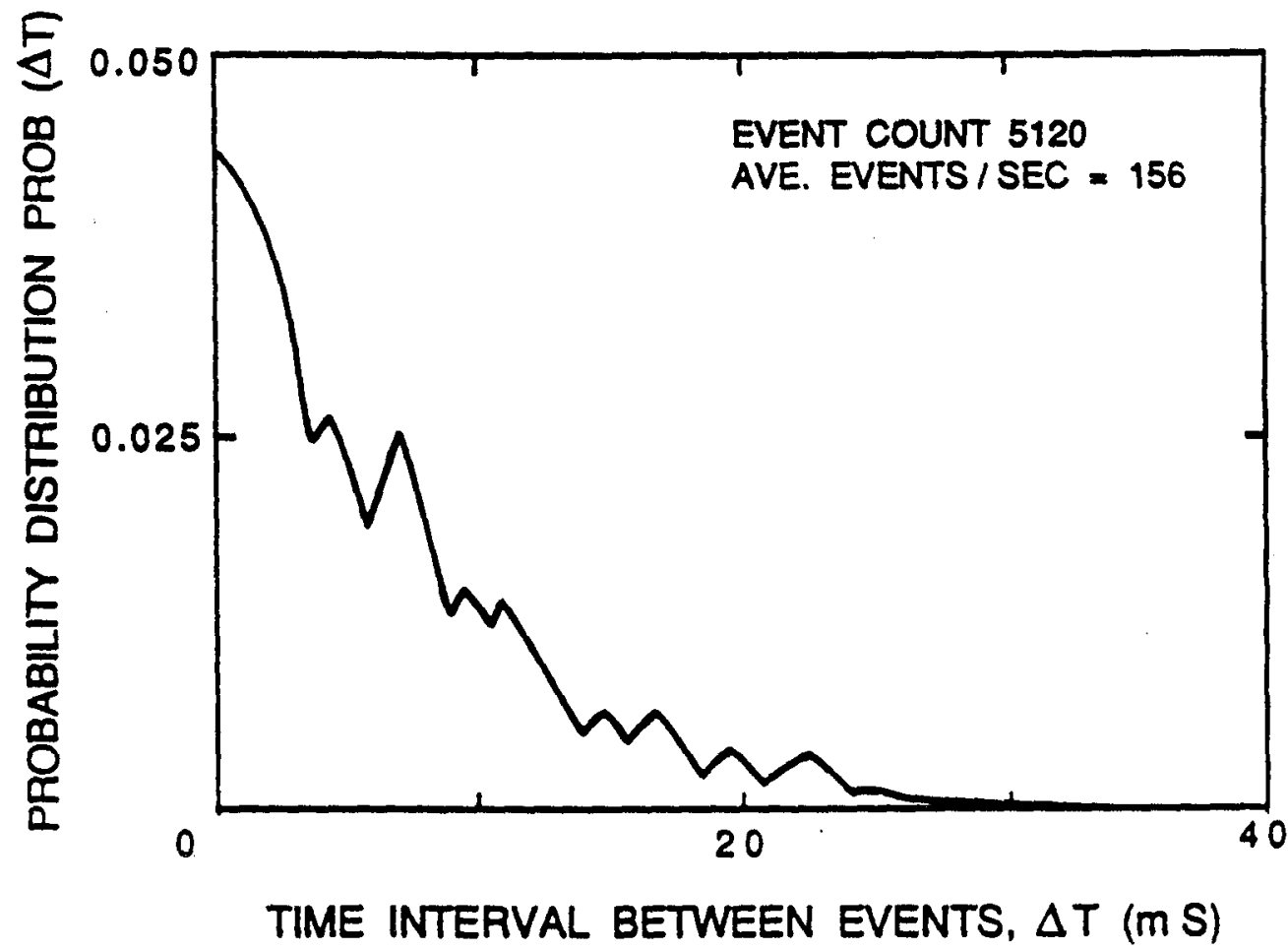

Figure 7.2 Typical distribution of time between cavitation events for cavitation on the Schiebe body. Data taken at $U=8.7 \mathrm{~m} / \mathrm{s}$ and $\sigma=0.45$. 


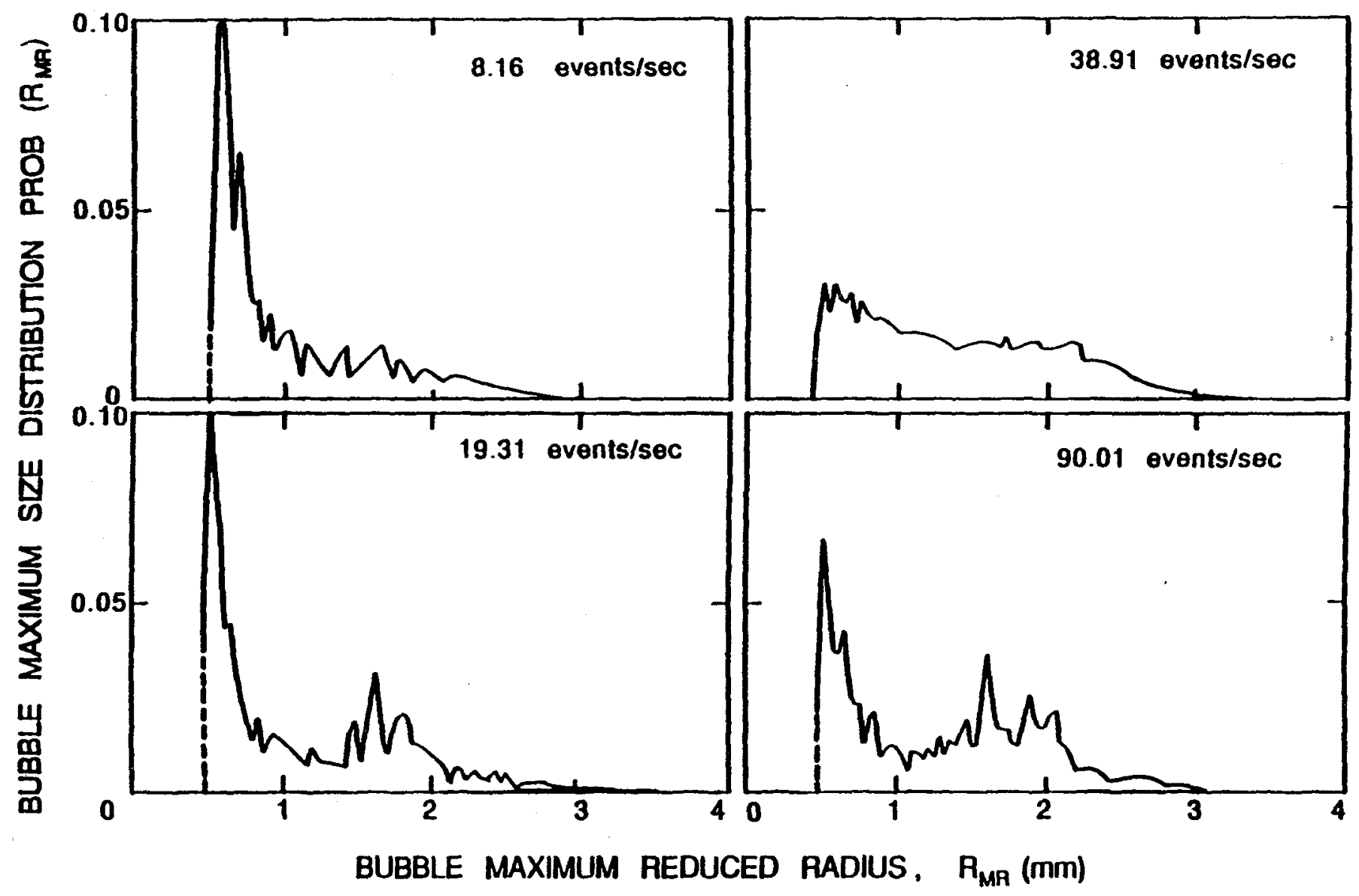

Figure 7.3 Example of four bubble maximum size distributions for a fixed free stream velocity and cavitation number for cavitation on the Schiebe body. 


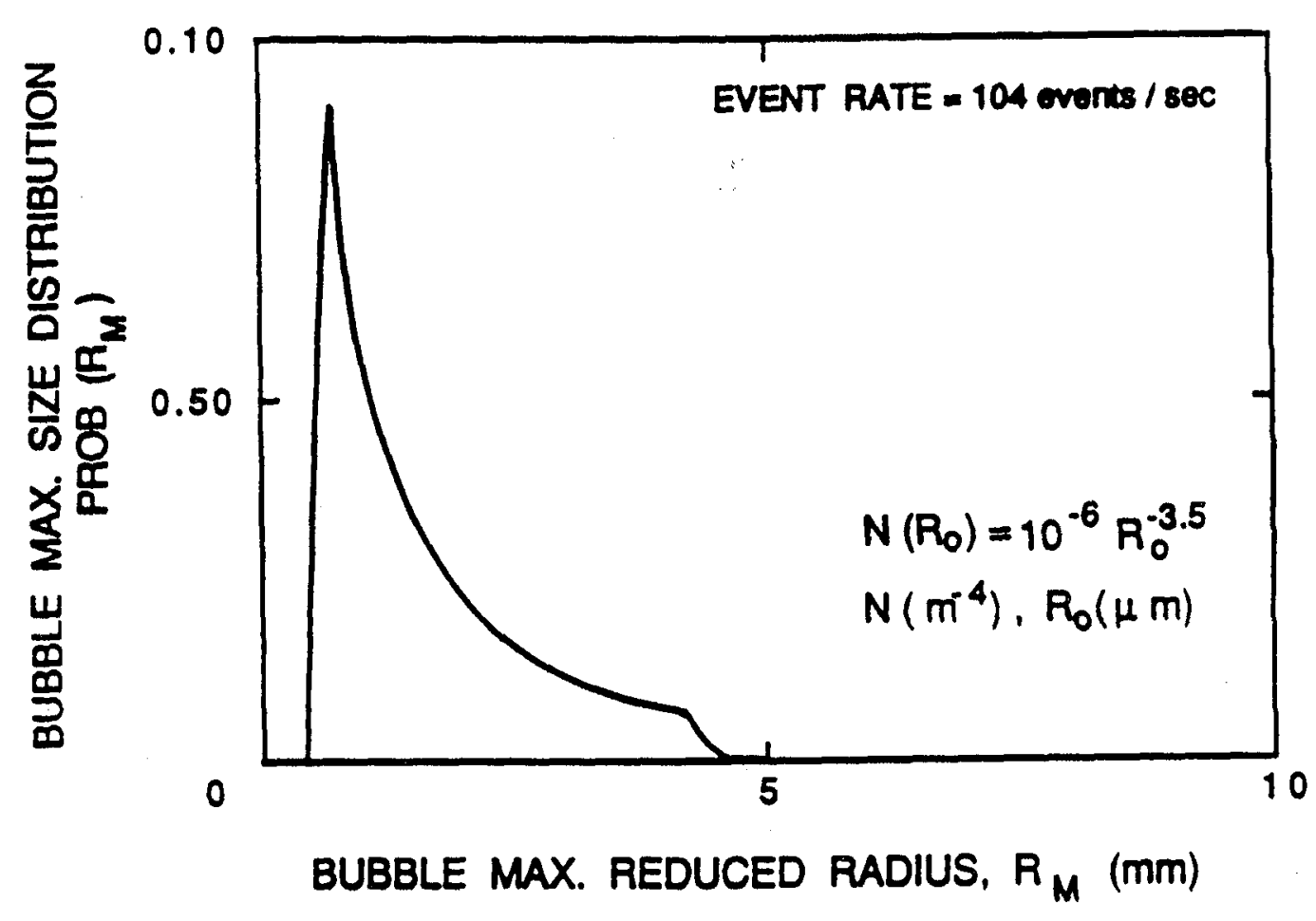

Figure 7.4 Calculated bubble maximum size distribution for cavitation generated on the I.T.T.C. body at $U=9 \mathrm{~m} / \mathrm{s}$ and $\sigma=0.45$ with a free stream nuclei number distribution given by $N\left(R_{0}\right)=10^{-6} R_{0}-3.5$ where $N$ is in $m^{4}$ and $R_{0}$ is in $\mu m$. 

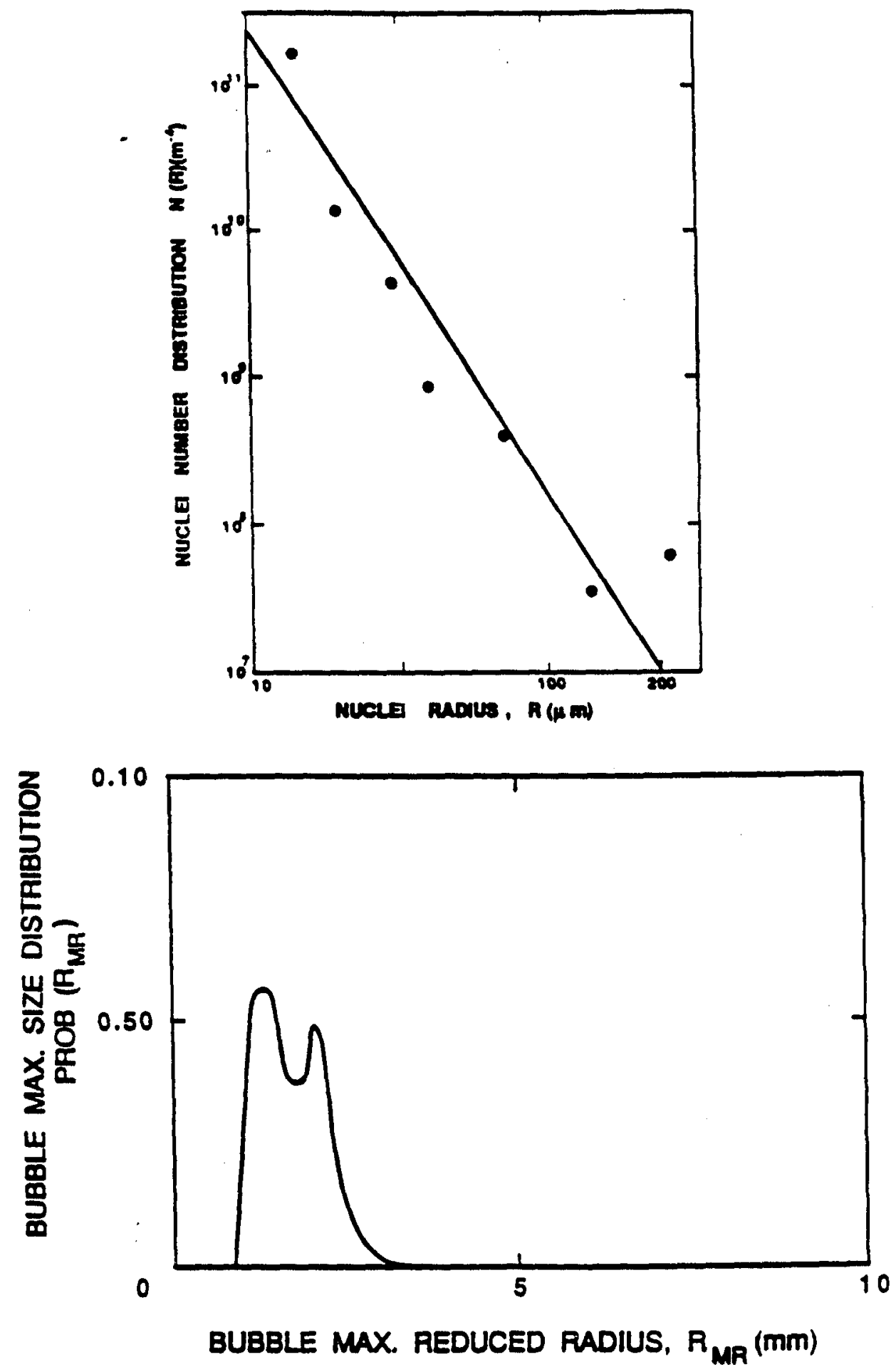

Figure 7.5 Measured nuclei number distribution, cavitation event rate, and bubble maximum size distribution for cavitation generated on the I.T.T.C. body at $U=9 \mathrm{~m} / \mathrm{s}$ and $\sigma=0.45$. 


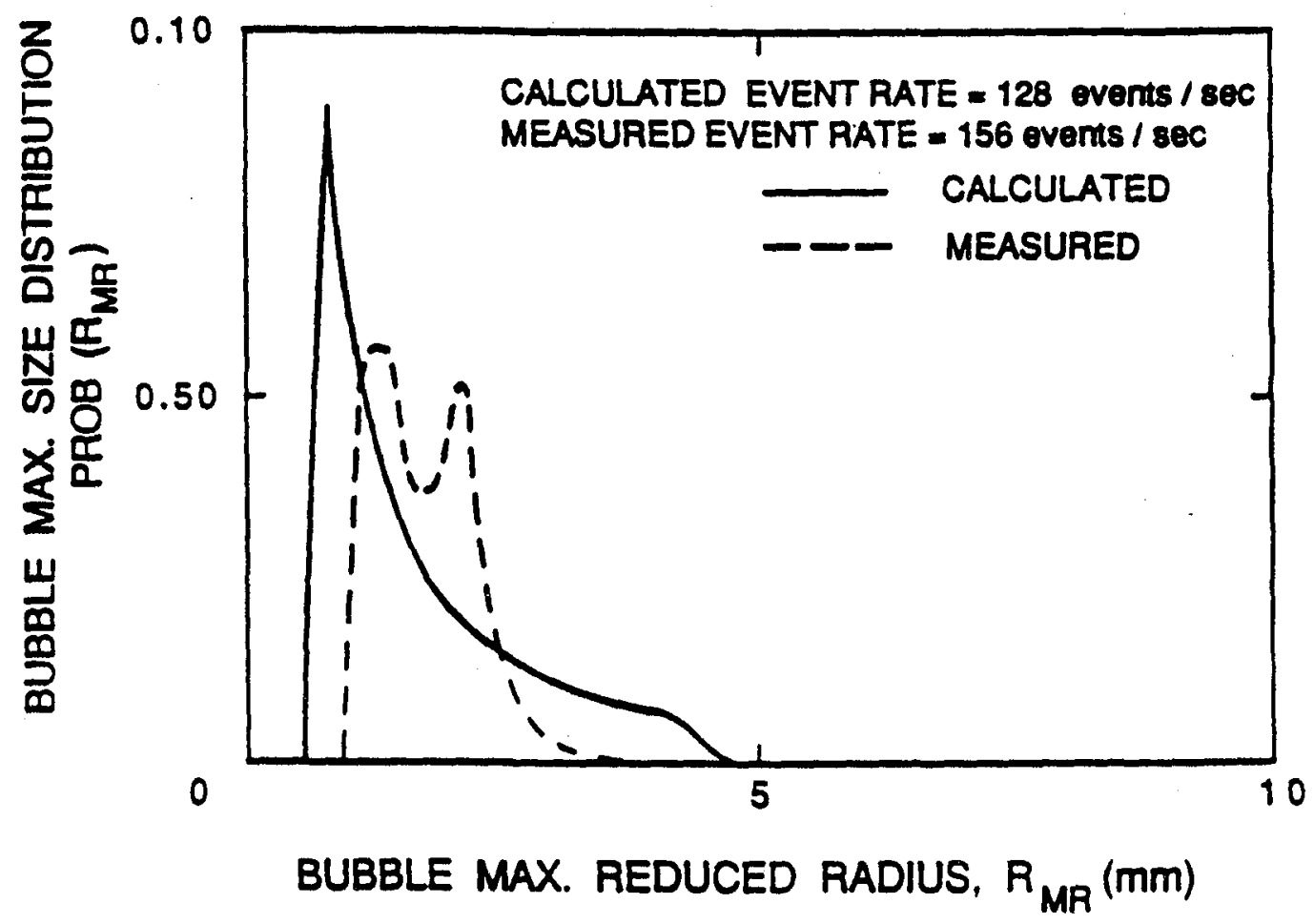

Figure 7.6 Calculated event rate and bubble maximum size distribution for cavitation on the I.T.T.C. headform at $U=9 \mathrm{~m} / \mathrm{s}$ and $\sigma=0.45$ using a correlation for the free stream nuclei number distribution derived from the data in Figure 7.5. 


\section{CHAPTER 8}

\section{Summary and Conclusion}

\subsection{Summary}

In this study a new passive electrical probe was used to study individual cavitation bubbles and cavitation event statistics measured on two axisymmetric headforms. The surface probe measures the changes in fluid conductivity caused by the presence of a bubble, and this change can be related to the position and volume of the bubble. For patch electrode geometries, the probe signal was used to isolate the location of single bubbles to facilitate their study, and circular electrodes were used to measure the volume of single bubbles and to compile cavitation event statistics.

The volume history of single, hydrodynamically produced cavitation bubbles were studied photographically, and these observations were related to the flow around the headform. As previous researchers have noted, bubbles forming near a surface generally assume a cap shape close to the headform surface. Only occasionally would quasi-spherical bubbles be observed to form over the headform surface, and these were most likely formed by the cavitation of large and infrequent nuclei.

The growth of the cavitation bubbles was similar for both the I.T.T.C. and Schiebe headforms. Nuclei grow into cap shapes as their expanding volume interacts with the headform surface, and the cap and wedge shapes formed by the bubbles near the point of maximum volume roughly correspond to the isobaric lines near the headform surface. As the bubbles grow they become elongated in the circumfrential direction as they acquire vorticity from the boundary layer.

Bubbles on the Schiebe body would collapse close to the headform surface, and they would often fission into vapor tubes prior to the final stages of collapse. On the other hand, bubbles on the I.T.T.C. body were observed to lift off the headform 
surface as they collapsed and to be stretched in the velocity gradient. The bubble would form a snout shape, which would result in a vapor tube collapsing above the body surface. After collapse, the bubbles on both headforms would rebound into a rough bubble or group of bubbles, which may collapse again or merely dissipate.

Cavitation bubbles were observed to interact with the laminar separation region of the I.T.T.C. headform. Although bubbles would ride over the separation bubble, the turbulence produced in the reattachment region would shear vapor of the underside off the cavitation bubbles. Also, bubbles would often induce local attached cavitation as they passed over the separation point for flows near the attached cavitation inception index. These attached cavities formed on the lateral edges of the bubble and briefly persisted even after the bubble had collapsed.

The noise generated by individual bubbles was recorded and related to the bubble growth and collapse mechanisms. Almost all of the noise emitted by the cavitation bubbles occurs during the violent first collapse and rebound. Previous researchers have suggested that the acoustic energy emitted by a collapsing cavitation bubble may be related to the bubble maximum volume, an indicator of the bubble stored energy, and this was found to be true. But, the relationship between the bubble maximum volume and the emitted acoustic impulse became indeterminate for bubbles of larger volume. Large bubbles may emit the expected level of acoustic energy, or they may be muted. Also, smaller bubbles would often collapse without any appreciable noise emission. Since noise is emitted during violent accelerations of the bubble volume, muted collapse mechanisms may not be coherent enough to produce concentrated bubble wall motions. An example of a disturbed collapse occurs when large bubbles on the I.T.T.C. body induced local cavitation. These bubbles would often produce no acoustic emission, which implies that the local attached cavitation disturbed the surrounding flow and disrupted the bubble collapse. Although many photographs were taken of collapsing bubbles, a reentrant microjet was not observed. Further study is needed to determine if the microjet is 
indeed absent and, if so, why.

The measured acoustic emission of single bubbles was compared to the noise emission calculated from the Rayleigh- Plesset equation. Even though the theoretical prediction was for spherically symmetric bubbles collapsing in an unbounded, incompressible fluid, the predicted acoustic impulses were relatively close to the measured impulses: the predicted impulses were larger than the measured impulses by a factor of about two for the I.T.T.C. body and a factor of six for the Schiebe body. The complex collapse mechanisms of the experimentally observed cavitation bubbles may be regarded as less efficient in their noise production than the ideal case of a spherically symmetric collapse. Also, the bubbles collapsing near the I.T.T.C. body have a more efficient mechanism than those of the Schiebe body. These differences may be related to differences in the details of collapse.

Acoustic pulses produced by the cavitation bubbles were individually recorded and spectrally analyzed to produce a composite acoustic spectrum. While the pulse shape is approximately predicted by theoretical considerations, bubble fission may lead to the production of multiple acoustic pressure peaks, which would alter the high frequency content of the spectrum. Also, many of the trends predicted for the bubble spectra are not observed experimentally. Instead, as other researchers have noted, the spectra of bubble cavitation noise is generally flat in the range of 10 to $100 \mathrm{kHz}$. More experimentation is needed to determine the high frequency bandwidth of the bubble acoustic emission.

Cavitation event statistics were measured and compared with the free stream nuclei number distribution. Bubble cavitation may be considered a stochastic process as a certain population of nuclei are swept over the cavitating body leading to a train of cavitation events and resulting noise pulses. The surface probe permits measurement of the maximum bubble size distribution and event rate statistics for moderately limited cavitation, and a simple model was formulated to relate these results to the holographically determined free stream nuclei population. 
The freestream nuclei number distribution was determined to be a highly variable parameter, even at nominally fixed tunnel operating conditions. Consequently, the number and size distribution of cavitating bubbles would drastically vary, often over the course of a single experiment. A knowledge of the nuclei population is essential for the detailed understanding of any cavitating flow, especially in the context of cavitation noise scaling, and better methods are needed to adequately quantify this parameter.

The relationship between the nuclei flux and the resulting cavitation event rate was successfully predicted by a simple model. This generally validates widely accepted nuclei stability criteria used in the model. The success of this model suggests that the tendency of a body to cavitate may be related to simple parameters derived from the non- cavitating flow around the body.

The bubble maximum size distribution, however, was not adequately predicted. The relationship between the pressure a nucleus experiences and the maximum volume it may attain is not adequately predicted by the Rayleigh-Plesset equation. This model does not consider the effects that the surface and surrounding flow have on the bubble growth except through the inviscid surface pressure distribution, and the maximum bubble sizes are consistently over- estimated. Study of the bubble maximum size distributions also reveals that the nuclei number distribution may have local maxima or minima that are not easily anticipated by the holographic measurements of nuclei number distributions.

\subsection{Conclusions}

This work provides an extensive experimental study of individual, naturally occurring cavitation bubbles and their acoustic emission. The results of this study indicate that the behavior of naturally occuring cavitation bubbles may depart drastically from that predicted by traditional theoretical models, which generally assume bubble sphericity. The shape and trajectory of cavitation bubbles near 
surfaces are quite complex and are signifcantly affected by the surrounding flow. In turn, the acoustic emission of a collapsing cavitation bubble will be related to the volume history of the bubble. Although many researchers have suspected these facts, this work provides the first detailed experimental observations.

Several questions are raised by these results. First, how would changes in scale and Reynolds number affect the results? Bubble dynamics would be expected to change with varying surface flows. Second, collapsing bubbles observed in this study were not seen to generate liquid microjets. Do bubbles that occur naturally in flows near surfaces generate microjets, and, if so, under what conditions? Also, the effects of bubble interactions may be explored.

As previous researchers have noted, knowledge of the nuclei number distribution is essential to the understanding of bubble cavitation results. The cavitation inception number, event rate, and bubble size distribution is directly related to the nuclei number distribution. With a knowledge of the free stream nuclei number distribution, the model used in this study adequately predicted the cavitation event rate and the approximate bubble maximum size distribution. This model was based on the non-cavitating flow around the body.

Further refinement of this model is certainly possible, and it may be extended to other shapes, such as hydrofoils. With a knowledge of the cavitation event rate and bubble maximum size distribution, the acoustic emission of the system may be estimated by using the results from individual bubbles. Alternately, a measurement of the cavitation event rate and bubble maximum size distribution may be used to infer the free stream nuclei number distribution. Although this idea of a "standard cavitator" is not new, the instrumentation used in this study may provide a new method of determining the cavitation susceptibility of a fluid. 


\section{REFERENCES}

Akulichev, V. A. and Ol'sheviskii, V. A. 1968. Relationship Between the Statistical Characteristics of Acoustic and Cavitation Noise. Soviet Physics-Acoustics, Vol. 14, pp. 22-26.

Arakeri, V. H. and Acosta, A. J. 1973. Viscous Effects in the Inception of Cavitation on Axisymmetric Bodies. ASME Journal of Fluids Engineering, Vol. 95, pp. 519-527.

Arakeri, V. H. and Shangumanathan, V. 1985. On the Evidence for the Effect of Bubble Interference on Cavitation Noise. Journal of Fluid Mechanics, Vol. 159 , pp. $131-150$.

Baiter, H. J. 1974. Aspects of Cavitation Noise. Symposium on High Powered Propulsion of Ships, Wageningen, The Netherlands, Publication No. 490, pp. 1-39.

Baiter, H. J. 1986. On Different Notions of Cavitation Noise and What They Imply. International Symp. on Cavitation and Multiphase Flow Noise, ASME FED Vol. 45, pp. 107-118.

Barker, S. J. 1975. Measurement of Radiated Noise is the Caltech High-Speed Water Tunnel-Part II: Radiated Noise from Cavitating Hydrofoils. Guggenheim Aeronautics Laboratory, California Insitute of Technology Report.

Benjamin, T. B. and Ellis, A. T. 1966. The Collapse of Cavitation Bubbles and the Pressures Thereby Produced Against Solid Boundaries. Philosophical Transactions of the Royal Society of London, Vol. 260, pp. 221-240.

Bernier, J. N. 1981. Unsteady Two-Phase Flow Instrumentation and Measurement. Rep. E200.4, California Institute of Technology, Division of Engineering and Applied Science.

Blake, J. R. and Gibson, D. C. 1987. Cavitation Bubbles Near Boundaries. Ann. 
Rev. Fluid Mechanics, Vol. 19, pp. 99-123.

Blake, W. K., Wolpert, M. J. and Geib, F. E. 1977. Cavitation Noise and Inception as Influenced by Boundary Layer Development on a Hydrofoil. Journal of Fluid Mechanics, Vol. 80, pp. 617-640.

Blake, W. K. 1986. Mechanics of Flow Induced Sound and Vibration. Volume 1, Chapter 6, Introduction to Bubble Dynamics and Cavitation, Academic Press, pp. 370-425.

Brennen, C. E. 1970. Cavity Surface Wave Patterns and General Appearance. Journal of Fluid Mechanics, Vol. 44, pp. 33-49.

Brennen, C. E. and Acosta, A. J. 1976. The Dynamic Transfer Function for a Cavitating Inducer. Journal of Fluids Engineering, Vol. 98, pp. 182-191.

Chahine, G. L., Courbiere, P. and Garnaud, P. 1979. Correlation Between Noise and Dynamics of Cavitation Bubbles. Sixth Conference on Fluid Machinery, Budapest, Vol. 1, pp. 200-209.

Cole, R. H. 1948. Underwater Explosions. Princeton University Press, pp. 28-33.

d'Agostino, L., Brennen, C. E. and Acosta, A. J. 1988. Linearized Dynamics of TwoDimensional Bubbles and Cavitating Flows Over Slender Surfaces. Journal of Fluid Mechanics, Vol. 199, pp. 155-176.

Dreyer, J. J. 1987. Free Stream Microbubble Effects on Travelling Bubble Cavitation Inception on the Schiebe Headform. ARL/PSU Technical Memorandum 87-205.

Ellis, A. T. 1952. Observations on Cavitation Bubble Collapse. Rep. 21-12, California Institute of Technology, Hydrodynamics Lab.

Ellis, A. T. 1966. On Jets and Shock Waves From Cavitation. Sixth Symposium on Naval Hydrodynamics. 
Epstein, P. S. and Plesset, M. S. 1959. On the Stability of Gas Bubbles in LiquidGas Solutions. Journal of Chemical Physics, Vol. 18, pp. 1505-1509.

Esipov, I. B. and Naugol'nyhk, K. A. 1973. Collapse of a Bubble in a Compressible Liquid. Soviet Physics-Acoustics, Vol. 19, pp. 187-188.

Fitzpatrick, H. M. and Stasberg, M. 1956. Hydrodynamic Sources of Sound. First Symposium on Naval Hydrodynamics, Washington, D. C., pp. 241-280.

Flynn, H. G. 1964. Physics of Acoustic Cavitation in Liquids. Physical Acoustics, editor W. P. Mason, Academic Press.

Gates, E. M. 1977. The Influence of Freestream Turbulence, Freestream Nuclei Populations, and Drag Reducing Polymer on Cavitation Inception on Two Axisymmetric Bodies. Rep. E182- 2, California Institute of Technology, Division of Engineering and Applied Science.

Gates, E. M., Billet, M. L., Katz, J., Ooi, K. K., Holl, W. and Acosta, A. J. 1979. Cavitation Inception and Nuclei Distribution - Joint ARL-CIT Experiments. Rep. E244-1, California Institute of Technology, Division of Engineering and Applied Science.

Gilmore, F. R. 1952. The Growth and Collapse of a Spherical Bubble in a Viscous Compressible Liquid. Rep. 26-4, California Institute of Technology, Hydrodynamics Lab.

Hamilton, M. F. 1981. Travelling Bubble Cavitation and Resulting Noise. Appl. Res. Lab., Penn. State, Tech. Mem. TM 81-76.

Hamilton, M. F., Thompson, D. E. and Billet, M. L. 1982. An Experimental Study of Travelling Bubble Cavitation and Noise. ASME International Symposium on Cavitation Noise, pp. 25-33.

Harrison, M. 1952. An Experimental Study of Single Bubble Cavitation Noise. Journal of the Acoustical Society of America, Vol. 28, pp. 776-782. 
Hentschel, W. and Lauterborn, W. 1982. Acoustic Emission of Single Laser Produced Cavitation Bubbles and Their Dynamics. Applied Scientific Research, Vol. 38, pp. 225-230.

Hickling, R. and Plesset, M. S. 1964. Collapse and Rebound of a Spherical Bubble in Water. Physics of Fluids, Vol. 7, pp. 7-14.

Hoyt, J. W. 1966. Wall Effect on I.T.T.C. Standard Head Shape Pressure Distribution. Contribution to 11th International Towing Tank Conference.

Il'ichev, V. I. 1968. Statistical Model of the Onset of Hydrodynamic Cavitation Noise. Soviet Physics-Acoustics, Vol. 14, pp. 22-26.

Ivany, R. D. and Hammitt, F. G. 1966. Cavitation Collapse in Viscous, Compressible Liquids-Numerical Analysis. Journal of Basic Engineering, Vol. 87, pp. 997-985.

Johnsson, V. E. and Hsieh, T. 1966. The Influence of Gas Nuclei on Cavitation Inception. Proc. Sixth Symposium on Naval Hydrodynamics, Washington D. C.

Katz, J. 1981. Cavitation Inception in Separated Flows. Rep. E183-5, California Institute of Technology, Division of Engineering and Applied Science.

Kirkwood, J. G. and Bethe, H. A. 1942. The Pressure Wave Produced by an Underwater Explosion. Rep. 588, Office of Research and Development.

Kling, C. L. and Hammitt, F. G. 1972. A Photographic Study of Spark Induced Cavitation Bubble Collapse. A.S.M.E. Journal of Basic Engineering, Vol. 94, pp. 825-833.

Knapp, R. T., Daily, J. W. and Hammitt, F. G. 1970. Cavitation. McGraw-Hill.

Knapp, R. T. and Hollander, A. 1948. Laboratory Investigations of the Mechanisms of Cavitation. Trans. ASME, July 1948, p. 419. 
Kimoto, H. 1987. An Experimental Evaluation of the Effects of a Water Microjet and a Shock Wave by a local Pressure Sensor. International Symposium on Cavitation Research Facilities and Techniques, ASME FED Vol. 57, pp. 217224.

Lauterborn, W. and Bolle, H. 1975. Experimental Investigation of CavitationBubble Collapse in the Neighborhood of a Solid Boundary. Journal of Fluid Mechanics, Vol. 72, pp. 391-399.

Lezzi, A. and Prosperetti, A. 1986. Bubble Dynamics in a Compressible Liquid, Part 1: First Order Theory. Journal of Fluid Mechanics, Vol. 168, pp. 457478.

Lezzi, A. and Prosperetti, A. 1987. Bubble Dynamics in a Compressible Liquid, Part 2: Second Order Theory. Journal of Fluid Mechanics, Vol. 185, pp. $289-321$.

Lindgren, H. and Johnsson, C. A. 1966. Cavitation Inception on Headforms: I.T.T.C. Comparative Experiments. Eleventh International Towing Tank Conference Proceedings, Tokyo.

Lyamshev, L. M. 1970. On the Theory of Hydrodynamic Cavitation Noise. Soviet Physics-Acoustics, Vol. 15, pp. 494-498.

Marboe, M. L., Billet, M. L. and Thompson, D. E. 1986. Some Aspects of Travelling Bubble Cavitation and Noise. International Symp. on Cavitation and Multiphase Flow Noise, ASME FED Vol. 45, pp. 119-126.

Mellon, R. H. 1956. An Experimental Study of the Collapse of a Spherical Cavity in Water. Journal Acoustic Society of America, Vol. 28, pp. 447-454.

Meyer, R. S., Billet, M. L. and Holl, J. W. 1989. Free Stream Nuclei and Cavitation. International Symp. on Cavitation Inception, ASME FED Vol. 89, pp. 55-62.

Miles, J. W. 1966. The Collapse Time of a Closed Cavity. Journal of Fluid 
Mechanics, Vol. 25, pp. 743-760.

Morch, K. A. 1982. Energy Consideration on the Collapse of Cavity Clusters. Applied Scientific Research, Vol. 38, p. 313.

Morozov, V. P. 1969. Cavitation Noise as a Train of Sound Pulses Generated at Random Times. Soviet Physics-Acoustics, Vol. 14, pp. 361-365.

Olsen, H. O. 1967. Theoretical and Experimental Investigation of Impedance Void Meters. Institute for Atomenergi, Kjeller Research Establishment, Kjellor, Norway.

Parkin, B. R. 1952. Scale Effects in Cavitating Flow. Ph.D. Thesis, California Institute of Technology.

Parkin, B. R. 1981. The Inception of Gaseous Microbubble Growth in Laminar Separation Bubbles. Journal of Fluids Engineering, Vol. 103, pp. 543-550.

Plesset, M. S. 1949. The Dynamics of Cavitation Bubbles. ASME Journal of Applied Mechanics, Vol. 16, pp. 228-231.

Plesset, M. S. and Chapman, R. B. 1970. Collapse of an Initially Spherical Vapor Cavity in the Neighborhood of a Solid Boundary. Rep. 85-49, California Institute of Technology, Division of Engineering and Applied Science.

Plesset, M. S. and Prosperetti, A. 1977. Bubble Dynamics and Cavitation. Annual Review of Fluid Mechanics, Vol. 9, pp. 145-185.

Rayleigh, Lord (Strutt, J. W.) 1917. On the Pressure Developed in a Liquid During the Collapse of a Spherical Cavity. Philosophical Magazine, Vol. 34, pp. 9498.

Rood, E. P. 1989. Mechanisms of Cavitation Inception. International Symp. on Cavitation Inception, ASME FED Vol. 89, pp. 1-22.

Schiebe, F. R. 1972. Measurement of the Cavitation Susceptibility of Water Using 
Standard Bodies. Rep. No. 118, St. Anthony Falls Hydraulic Laboratory, University of Minnesota.

Van der Meulen, J. H. J. 1980. Boundary Layer and Cavitation Studies of NACA 16-012 and NACA 4412 Hydrofoils. Thirteenth Symposium on Naval Hydrodynamics, Tokyo.

Van der Meulen, J. H. J. and van Renesse, R. L. 1989. The Collapse of Bubbles in a Flow Near a Boundary. Seventeenth Symposium on Naval Hydrodynamics, The Hague.

Weitendorf, E. A. 1989. 25 Years of Research on Propellor Excited Pressure Fluctuations and Cavitation. Proc. Third Int. Symp. on Cavitation Noise and Erosion in Fluid Systems, San Francisco, Dec. 1989, pp. 1-10. 


\section{Appendix A \\ Technical Description of the Surface Electrode Probe}

\section{A.1 Introduction}

This appendix provides a technical description of the surface electrode probe. The system may be divided into three major sections: the electrode bridge, the demodulator, and the system controller (Figure A.1). The front and back panel of the electrical system assembly is shown in Figure A.2, and the circuit board placement is shown in Figure A.3. Each system will be described separately with accompanying circuit drawings.

\section{A.2 The Electrode Bridge}

The surface electrode probe detects cavitation by measuring small changes in fluid impedance, and the electrode bridge is the circuit that accomplishes this task. Figure A.4 shows a block diagram of the bridge, Figure A.5 shows an electrical schematic of the bridge circuit, Figure A.6 shows the printed circuit board layout, and Figure A.7 shows the component layout. The three main sections of the bridge are the signal source, the passive bridge, and the instrumentation amplifier.

The electrode bridge measures small changes in output impedance at a single electrode. A sinusoidel, constant amplitude voltage is applied to the electrode, and a base current will be generated in the fluid. In order to complete the current loop through the fluid, a current sink must be provided, and this may be accomplished by installing a second electrode somewhere in the flow system. Usually, multiple electrodes are used, and by adjusting the voltages and phases at each electrode, the electric field may be manipulated and almost all of the generated current will be sinked within the electrode system.

The voltage source generates a low output impedance voltage that may be applied to the electrode. The base carrier signal is modulated to change the voltage amplitude and adjust the phase either 0 or 180 degrees, and this signal is amplified 
to produce a low output impedance voltage source that is applied to the measuring electrode. Two additional amplifiers are provided to drive the shield electrode, which may be used to modify the electric field of the measuring electrode.

A current sensing resistor is inserted into the feedback loop of the measuring electrode amplifier. Changes in the voltage across this resistor are used to measure the changes in the current emitted by the measuring electrode. The magnitude of this resistor will determine the bridge sensitivity: as the resistance increases, small changes in the electrode current will produce larger voltage differences. For the case of void free fluid, the base condition, a steady rms current will be emitted by the electrode. The passive bridge is used to null the voltage signal produced by this base current. Then small changes in the electrode current can be amplified to produce the probe signal.

In the base condition, the voltage difference measured by the instrumentation amplifier is nulled to zero by adjusting the resistive and capacitive elements of the passive bridge. Also, a voltage controlled resistor is provided to allow for automatic zeroing of the bridge, which will be described below. The exact value and range of the passive bridge components are strongly influenced by the bulk conductivity of the fluid, electrode voltages, and electrode geometry.

After the passive bridge is nulled, small changes in the electrode current will produce a change in the voltage difference measured by the instrumentation amplifier. This difference is amplified and the result is an a.c. signal whose amplitude is proportional to the change in electrode current.

\section{A.3 The Demodulator}

The amplitude of the bridge signal is recovered with the demodulator. Figure A.8 is a block diagram of the demodulator, Figure A.9 is an electrical schematic diagram, Figure A.10 is the printed circuit board layout, and Figure A.11 is the component layout, Demodulation is achieved by multiplying the bridge signal with 
a carrier signal and filtering the product. If two a.c. coupled sinusoidal signals of the same frequency and phase are multiplied, the result will be a signal with twice the original frequency and a d.c. offset. The high frequency portion of the multiplied signal may be filtered off, and the result is a signal proportional to the product of the two original amplitudes. In this case, these two signals are the bridge signal and a carrier signal of constant amplitude and phase.

Before demodulation, the bridge signal is band-pass filtered. Also, the carrier signal is phase shifted to make its phase relative to the bridge signal either 0 or 180 degrees. These signals are multiplied and filtered to produce a signal proportional to the changes in fluid impedance.

Four signals are derived from the demodulator sub- system. The first is the a.c. bridge signal, which is the amplified and filtered signal from the bridge subsystem. This signal is used to null the bridge. The resulting demodulated signal is output, and this signal is both high-pass and low-pass filtered to separate the mean and fluctuating portions of the electrode signal. A multiplexer is provided to route these four signals from the board.

\section{A.4 Control System}

Because multiple sixteen bridges are implemented, a control system is necessary to manage the individual electrode voltages and bridge signals. The control system has three major components: the carrier generator, control voltage generator, and computer interface. Figure A.12 is a block diagram of the control interface. Figure A.13 is an electrical schematic diagram of the bus interface, Figure A.14 is an electrical schematic diagram of the system interface, Figure A.15 is an electrical schematic diagram of the zeroing voltage circuit, Figure A.16 is an electrical schematic of the reference voltage circuit, and Figure A.17 is an electrical diagram of the carrier generator.

The bridge was designed to be computer controlled. However, panel switches 
could be used to overide computer commands. The computer was used to select which of the four possible signals from the demodulator board would be multiplexed to the output ports. Also, the computer was used to set the two control voltages needed by each bridge: one to set the electrode voltage and one to fine zero the bridge. Interaction with the computer was achieved with a standard I.B.M. PC bus interface. Figure A.18 provides a list of the digital codes used to interface with the control system. The voltage controls were generated with two sets of digital to analog convertors (DACs). Each bridge had two DACs dedicated to produce the desired voltage. The carrier was generated with an integrated oscillator. 


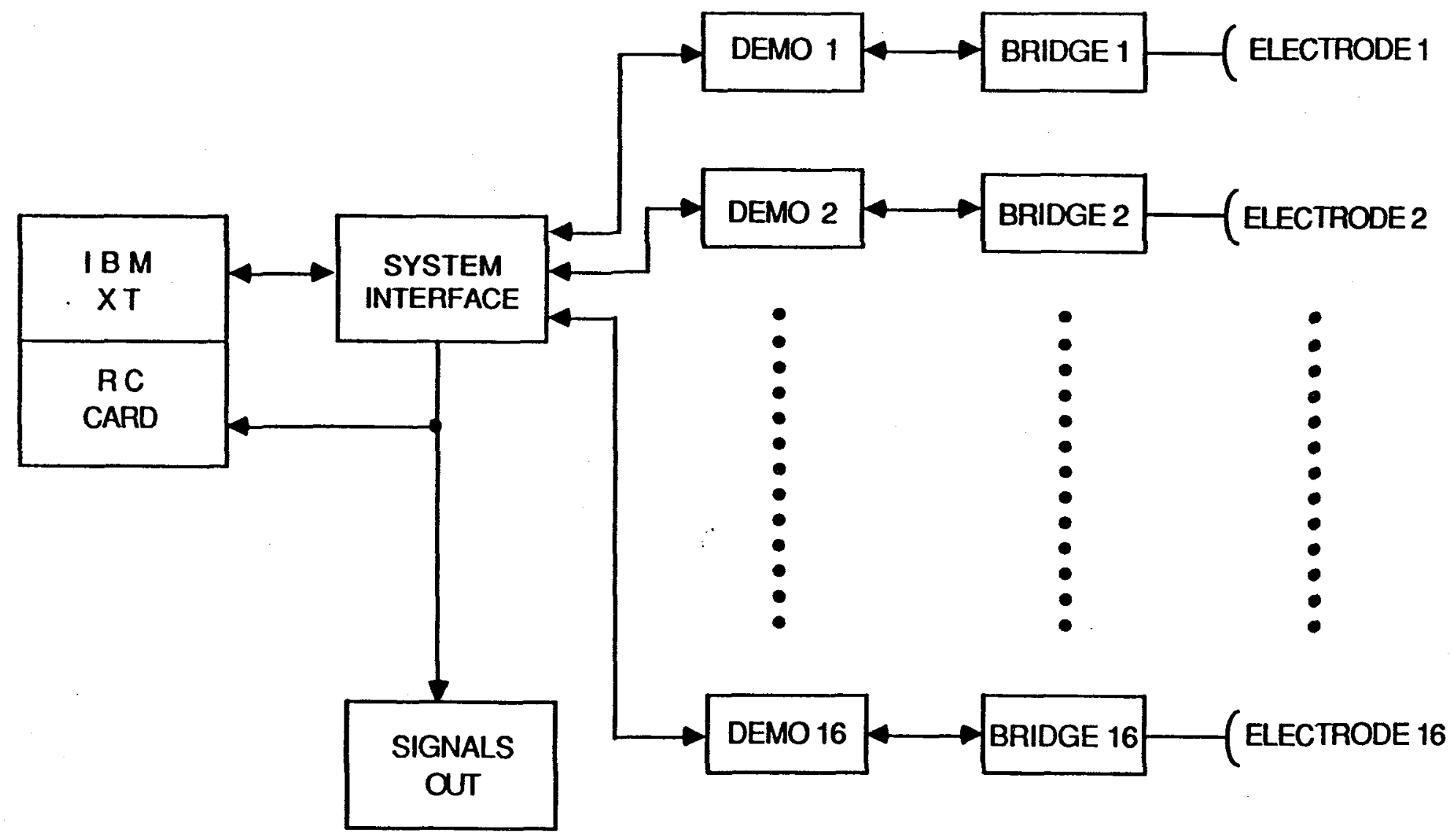

Figure A.1 Block diagram of the surface electrode system. 
- signal ac 、

- signal - - - $\quad \begin{array}{llllllll}1 & 2 & 3 & 4 & 5 & 6 & 7 & 8 \\ 0 & 0 & 0 & 0 & 0 & 0 & 0 & 0\end{array}$

- signal DC

- BRIDGe - $-\theta \quad \begin{array}{cccccccc}9 & 10 & 11 & 12 & 13 & 14 & 15 & 16 \\ 0 & 0 & 0 & 0 & 0 & 0 & 0 & 0\end{array}$ NULL

- panel - $\theta$ o cafrier $\quad$ O 0

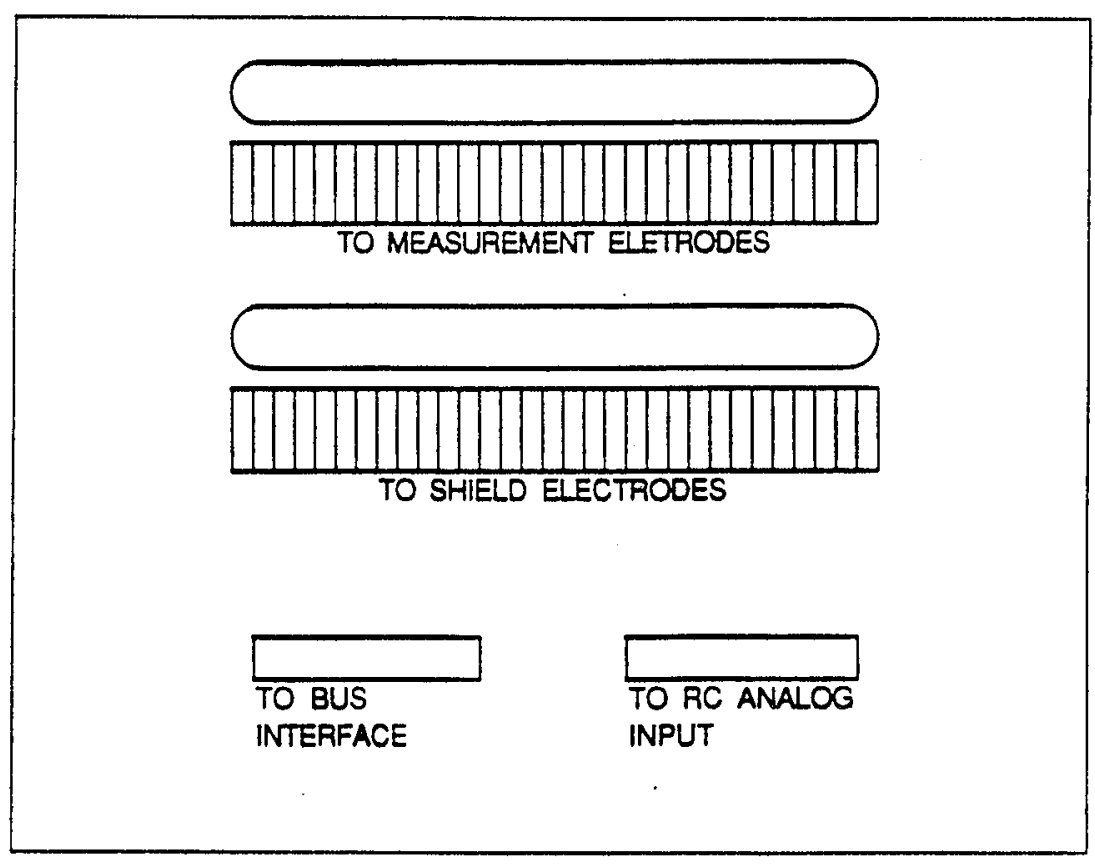

Figure A.2 Front and back panel of the probe assembly. 


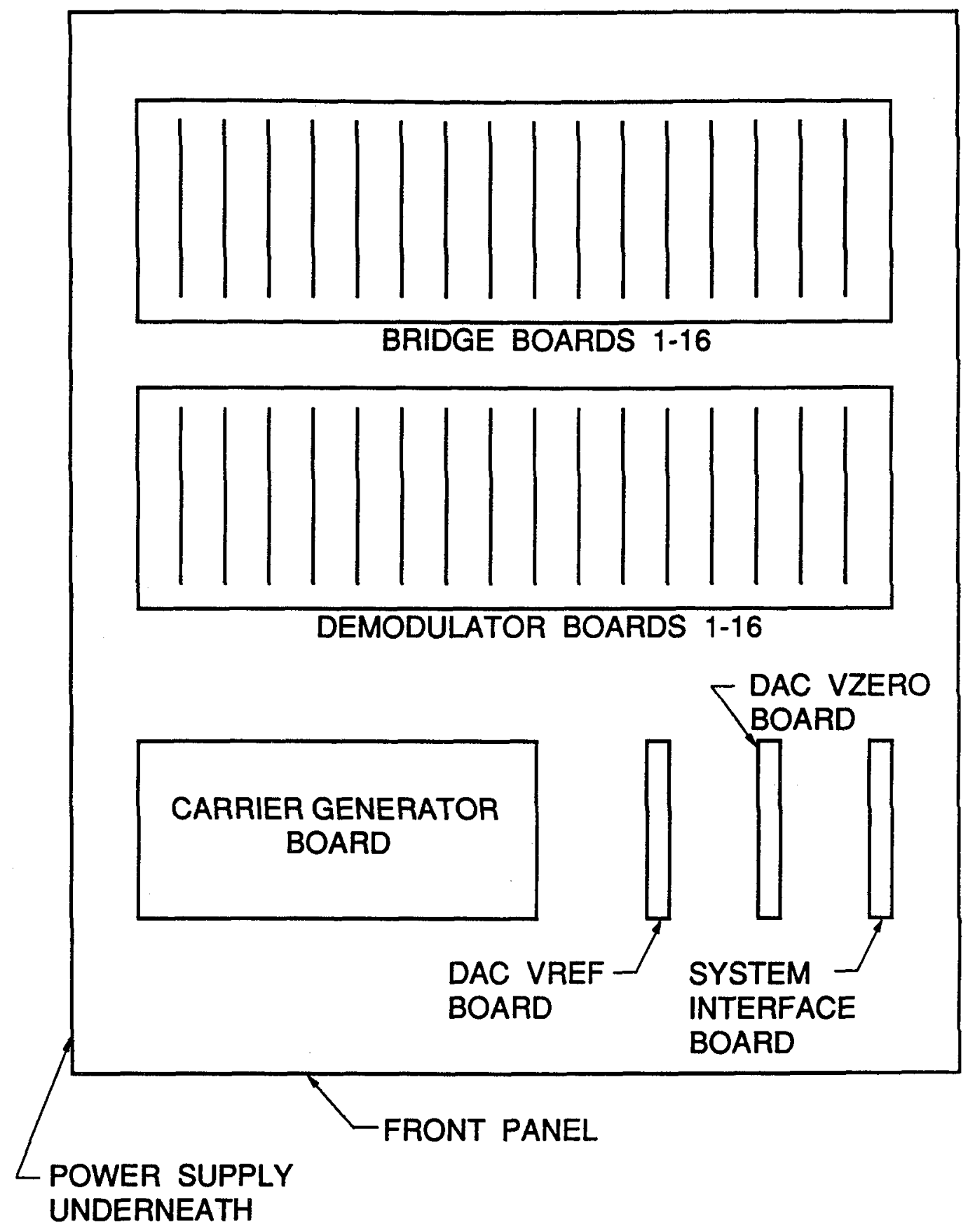

Figure A.3 Circuit board layout of the probe assemmbly. 


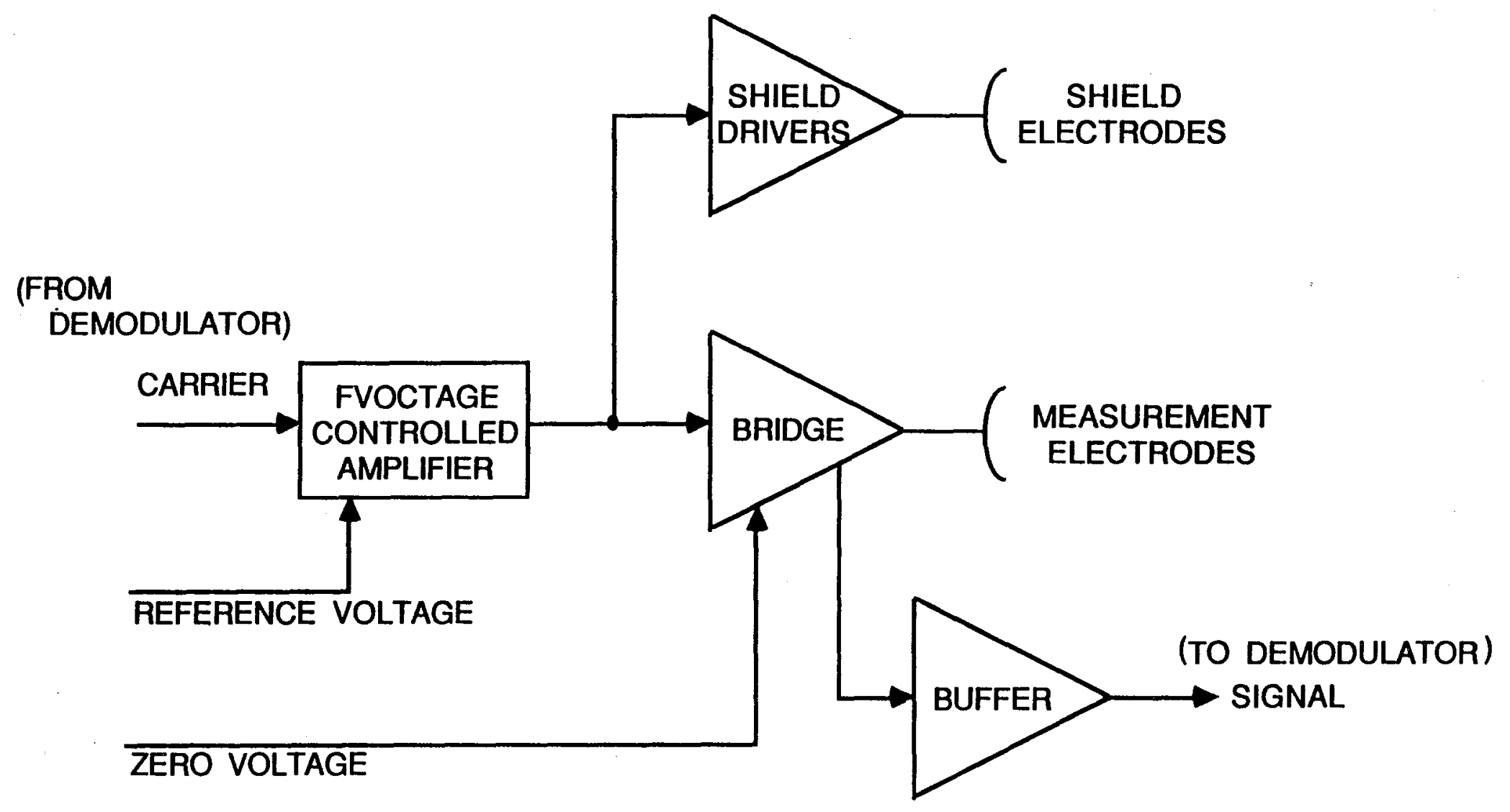

Figure A.4 Block diagram of the electrode bridge. 
ALL POWER LINES BYPASSED WITH 0.1 U CERAMIC CAPS

(TANTALUM, WHERE NECESSAGY)

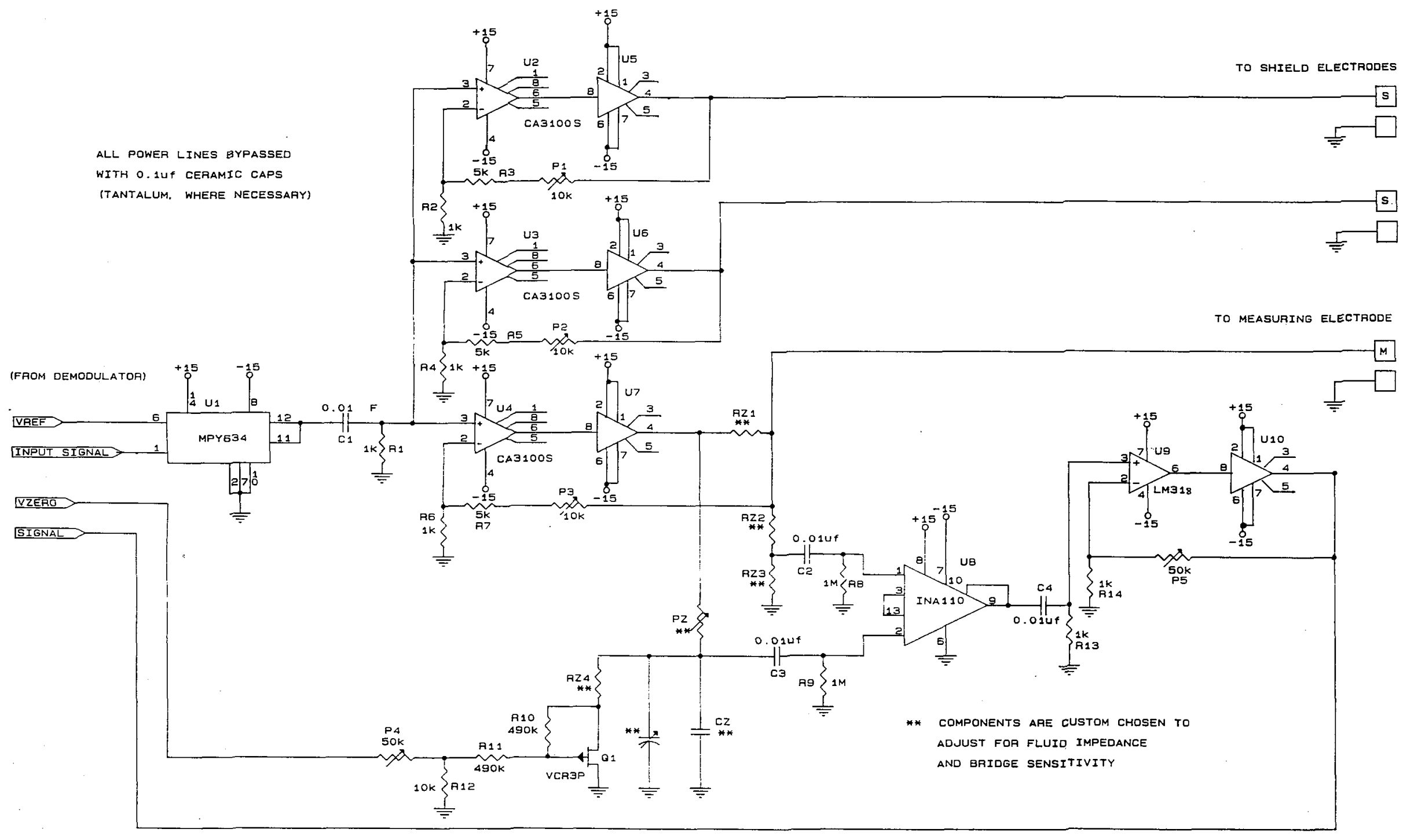

Figure A.5 Electrical schematic diagram of the electrode bridge. 

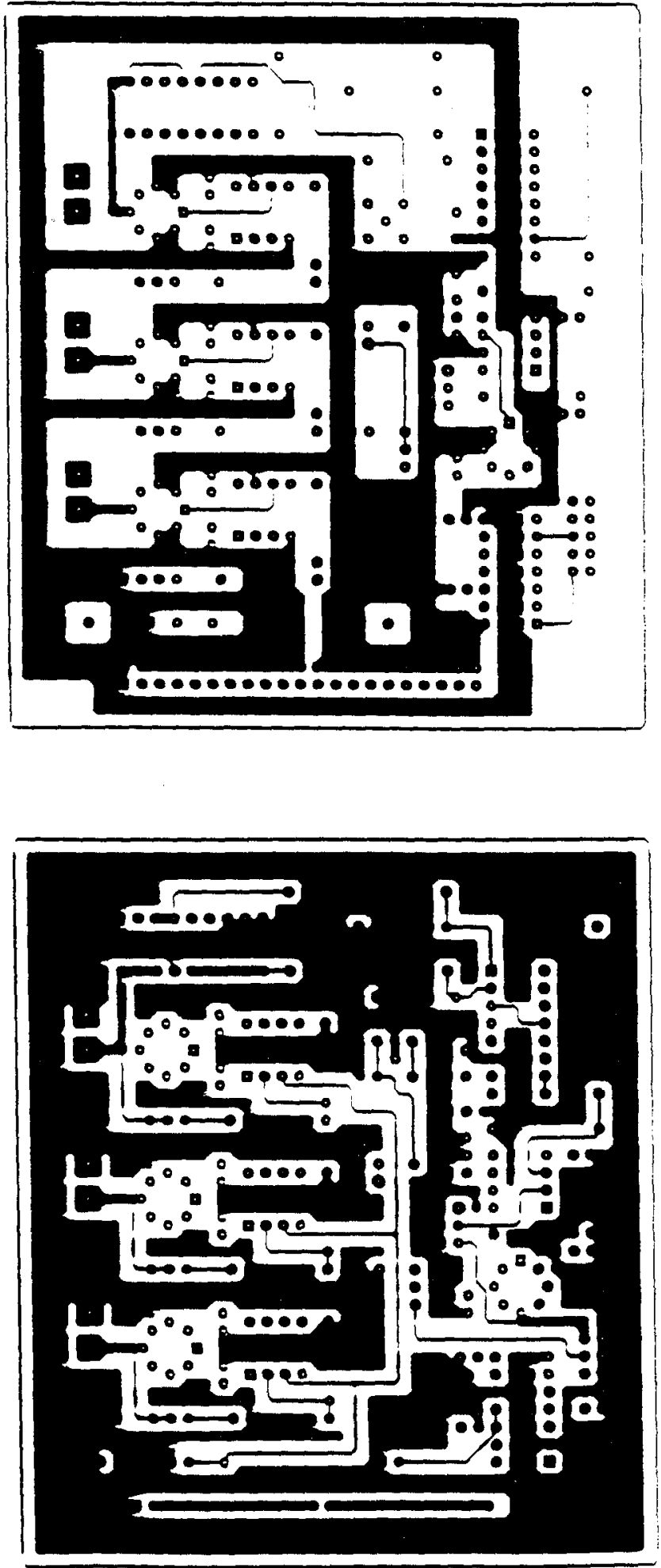

Figure A.6 Printed circuit board layout for electrode bridge. 


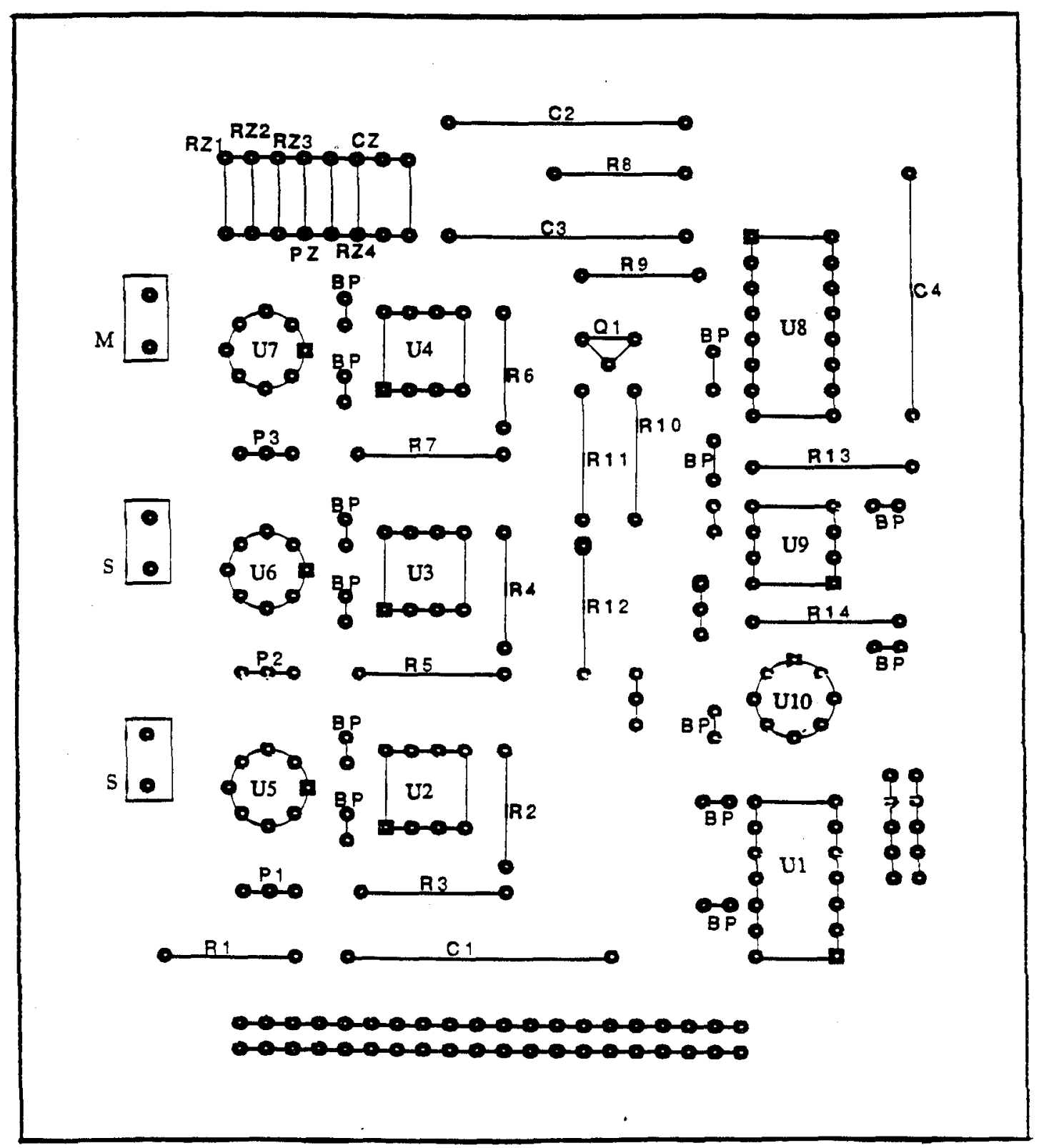

Figure A.7 Component layout for the electrode bridge board. 


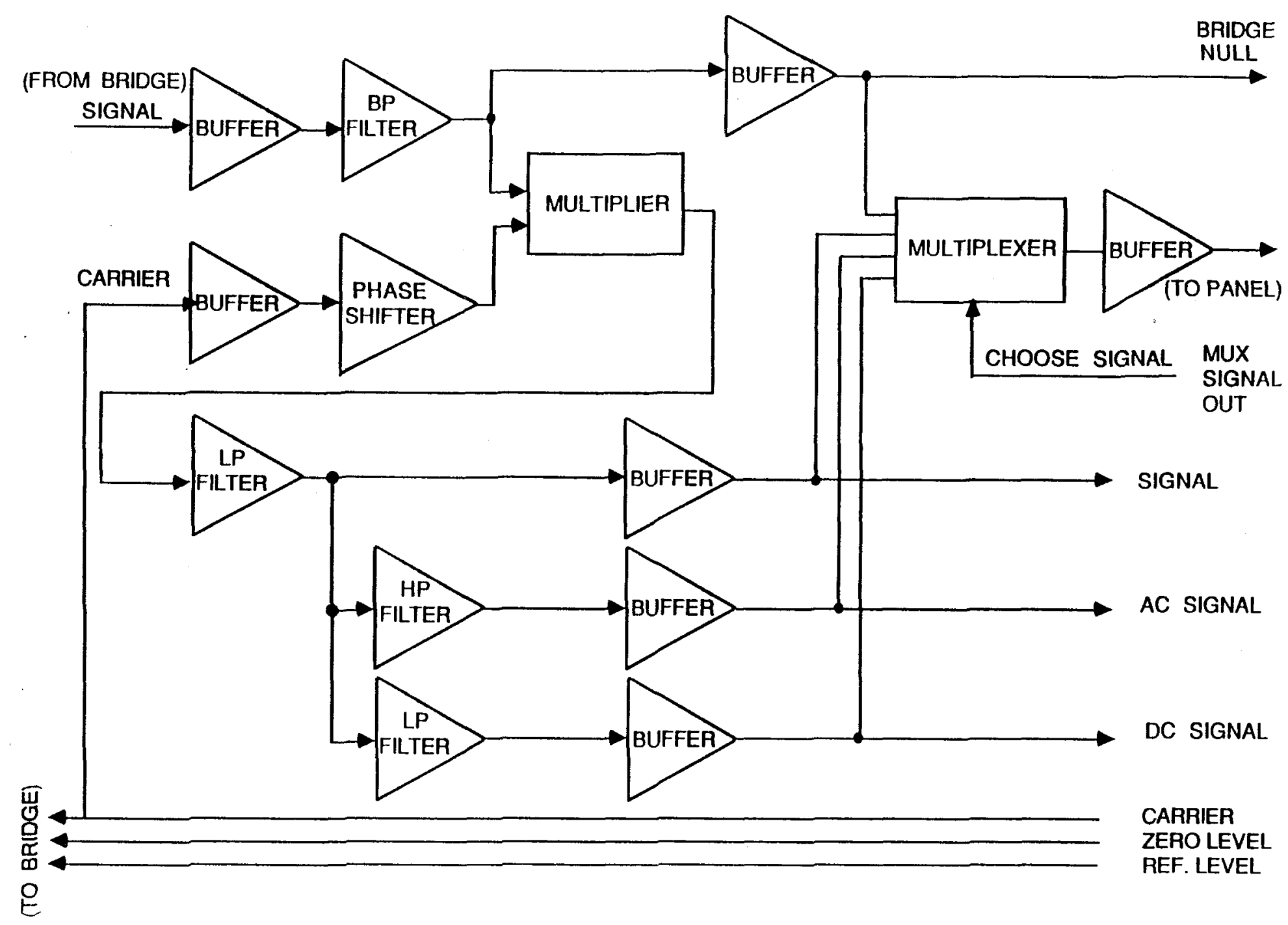

Figure A.8 Block Diagram of the demodulator. 


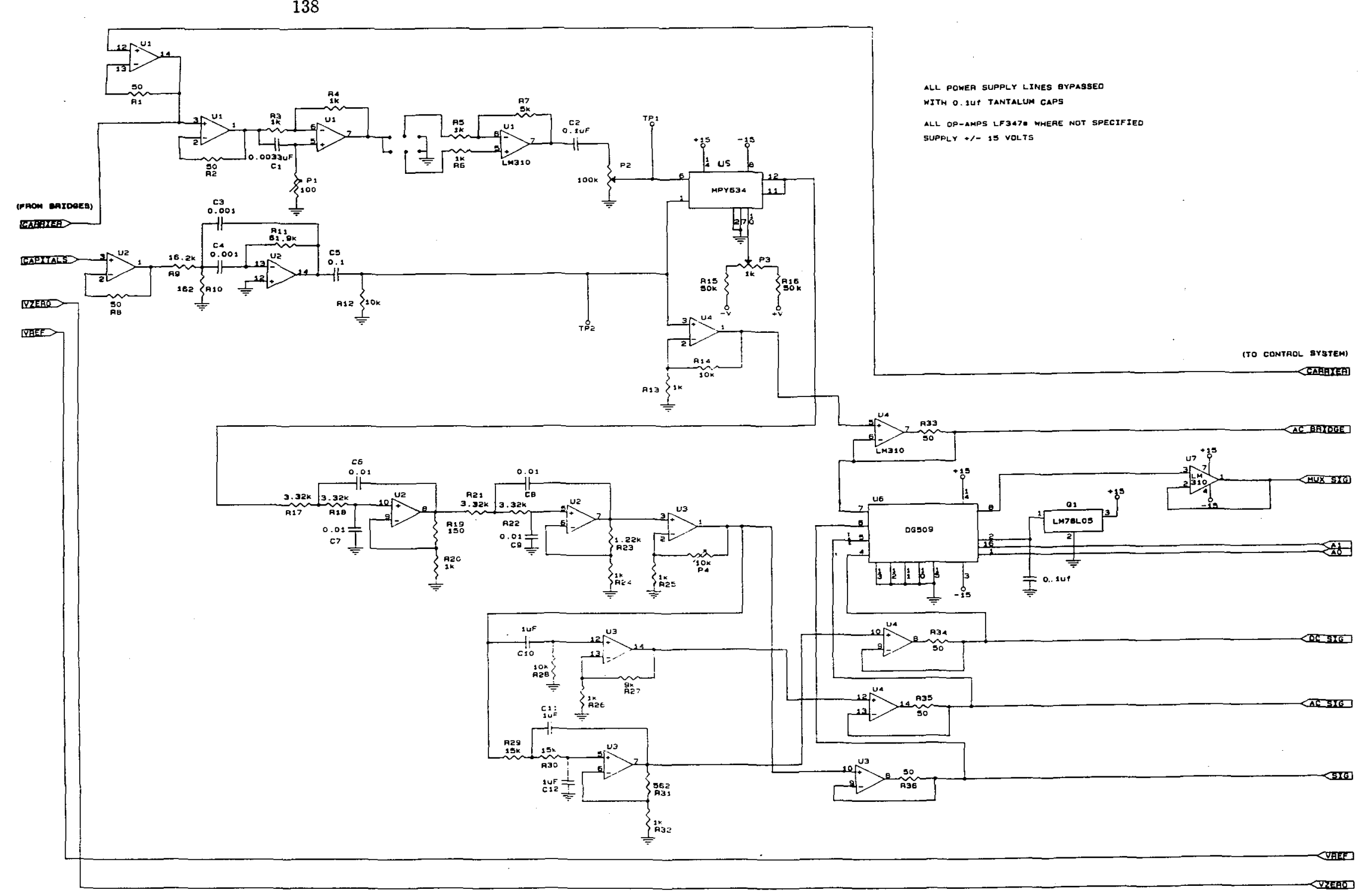

Figure A.9 Electrical schematic diagram of the demodulator. 

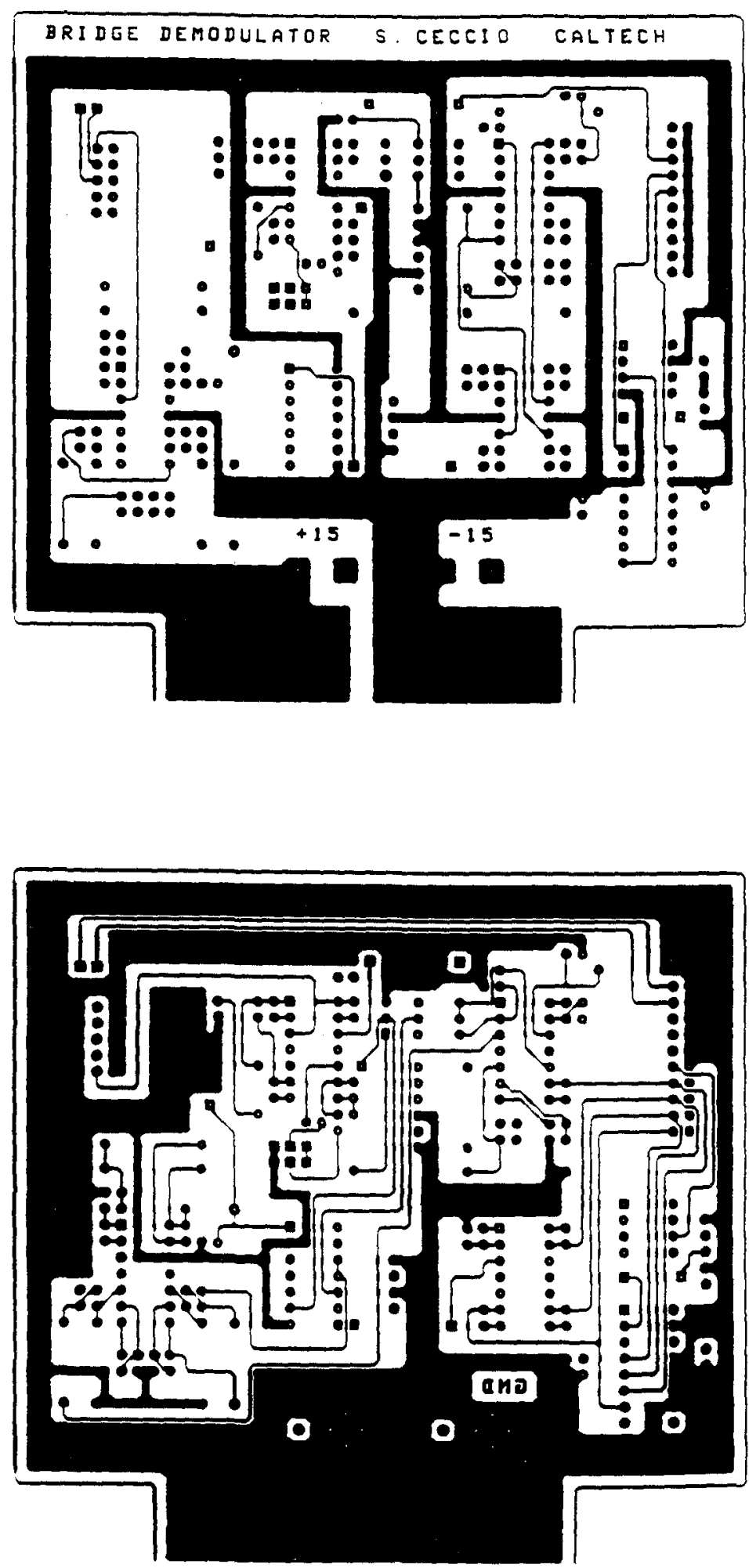

Figure A.10 Printed circuit board layout for the demodulator. 


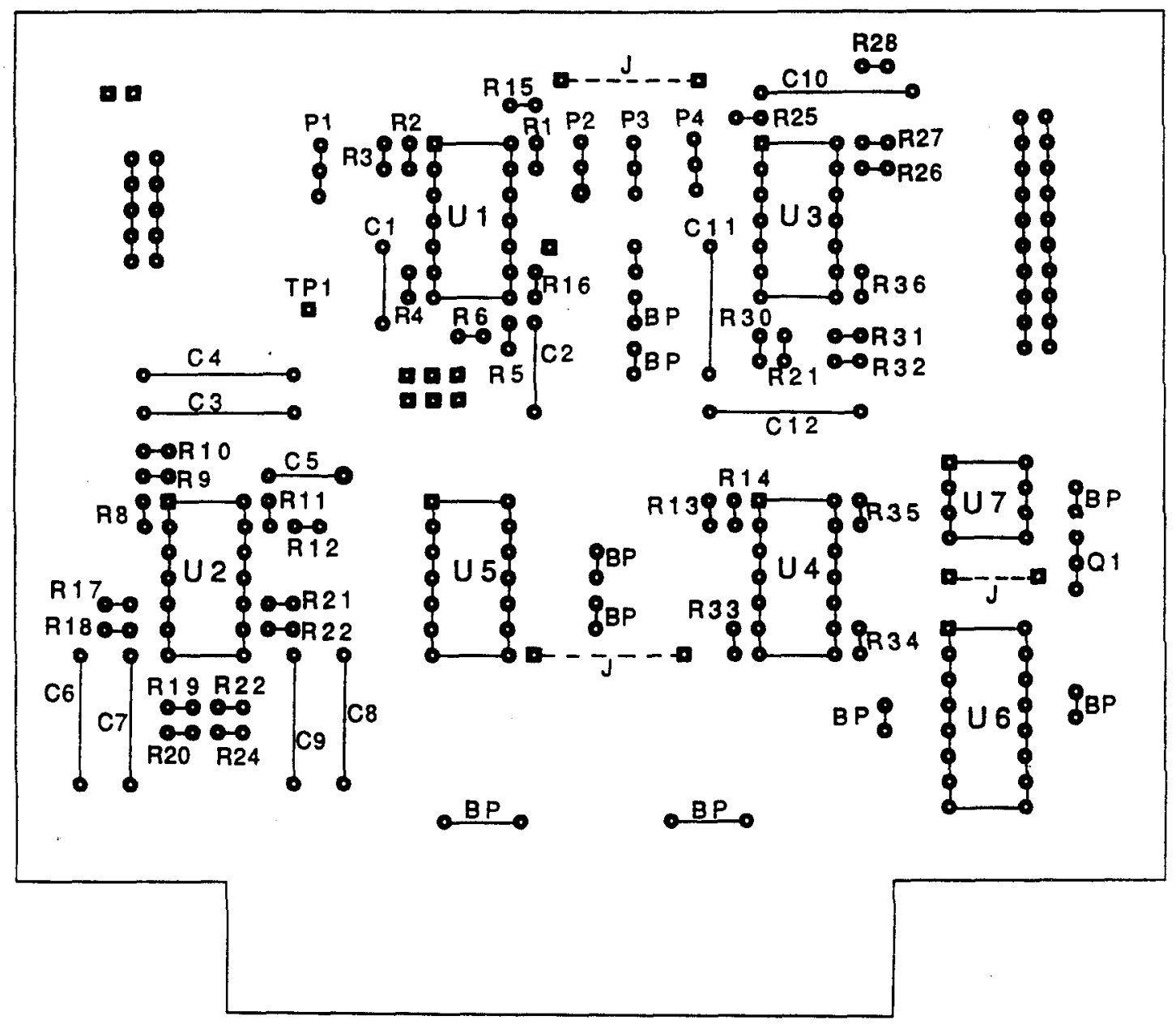

Figure A.11 Component layout for the demodulator board. 


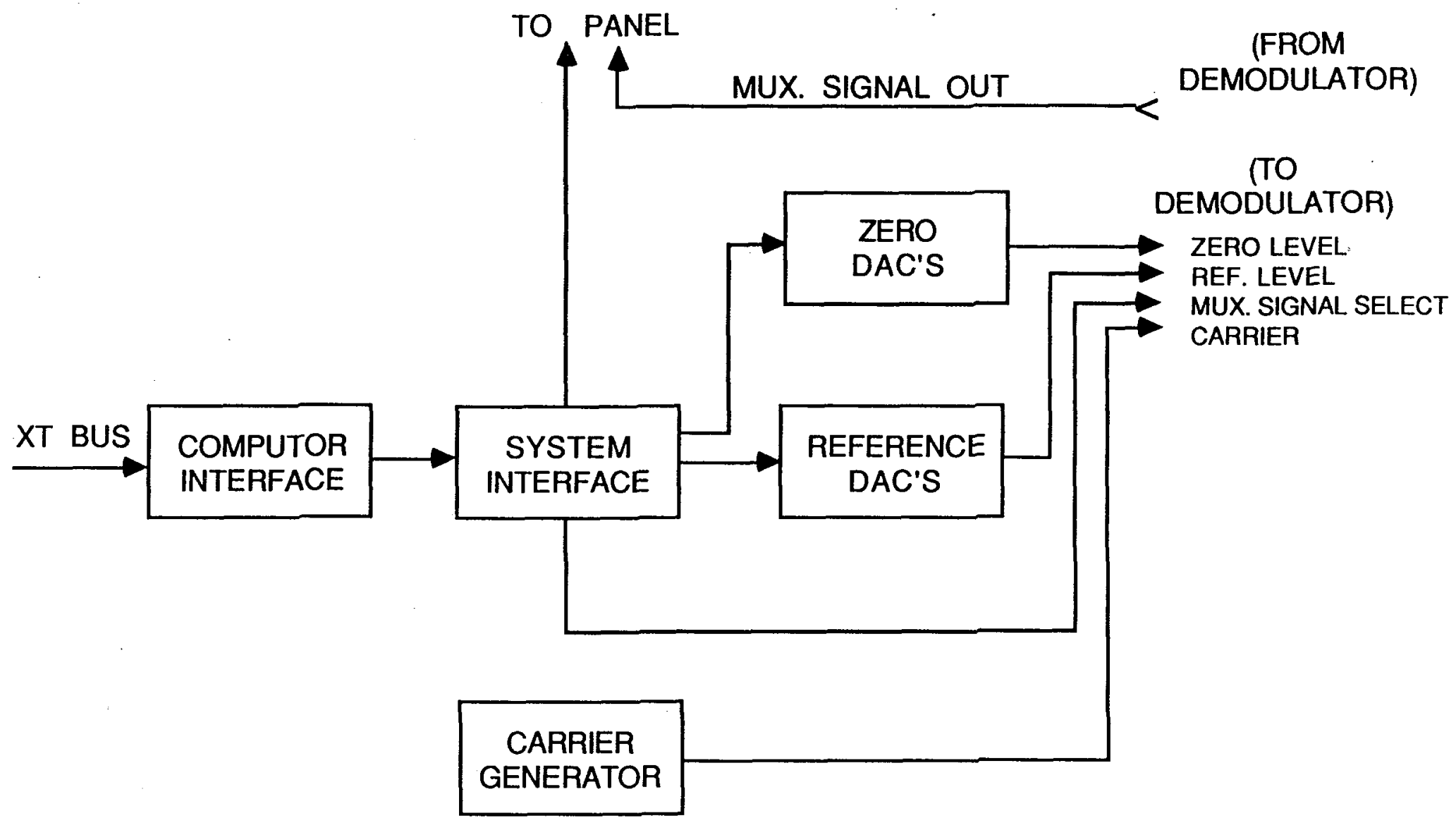

Figure A.12 Block Diagram of the control system. 


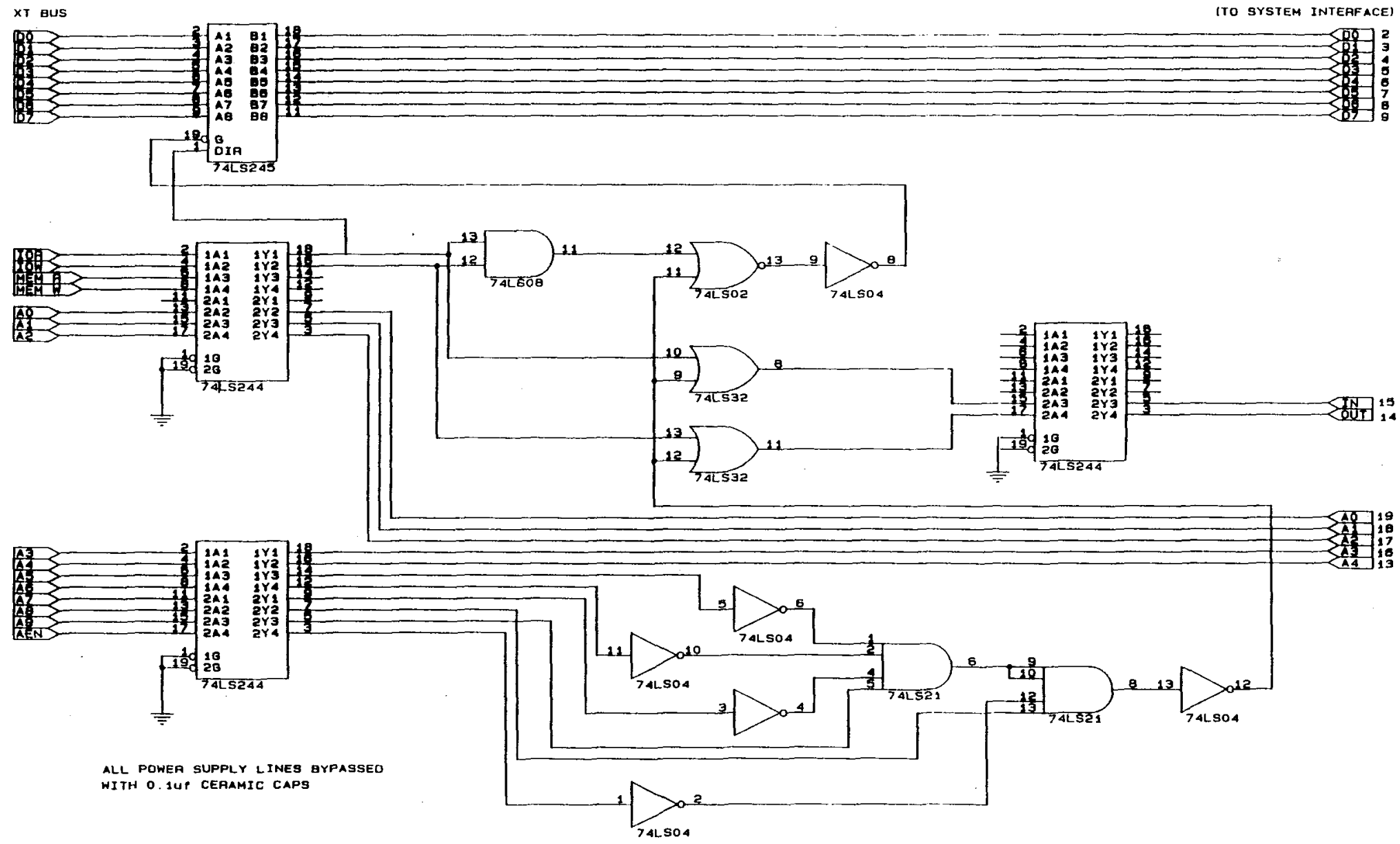

Figure A.13 Electrical schematic diagram of the bus interface. 


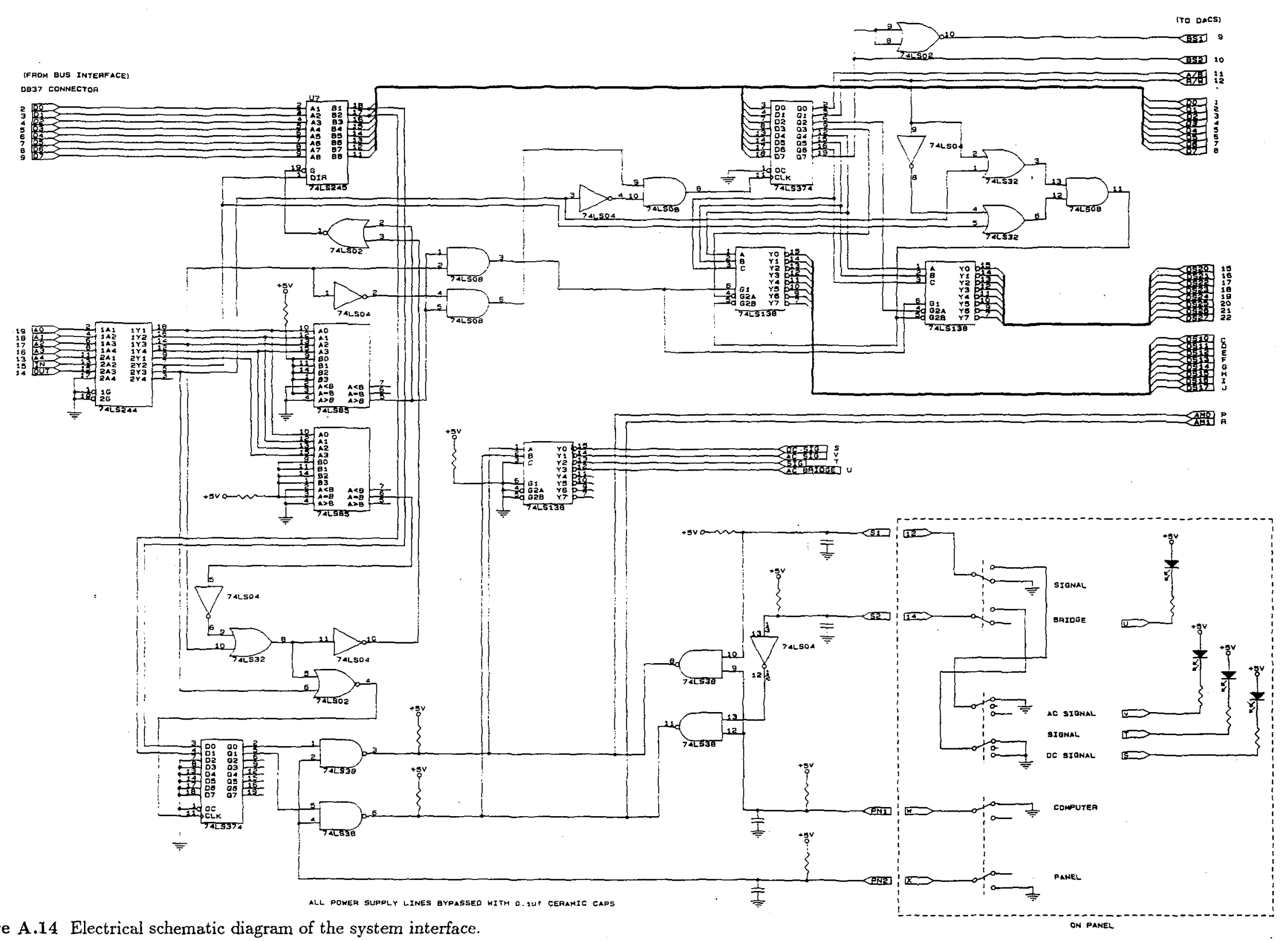

Figure A.14 Electrical schematic diagram of the system interface. 


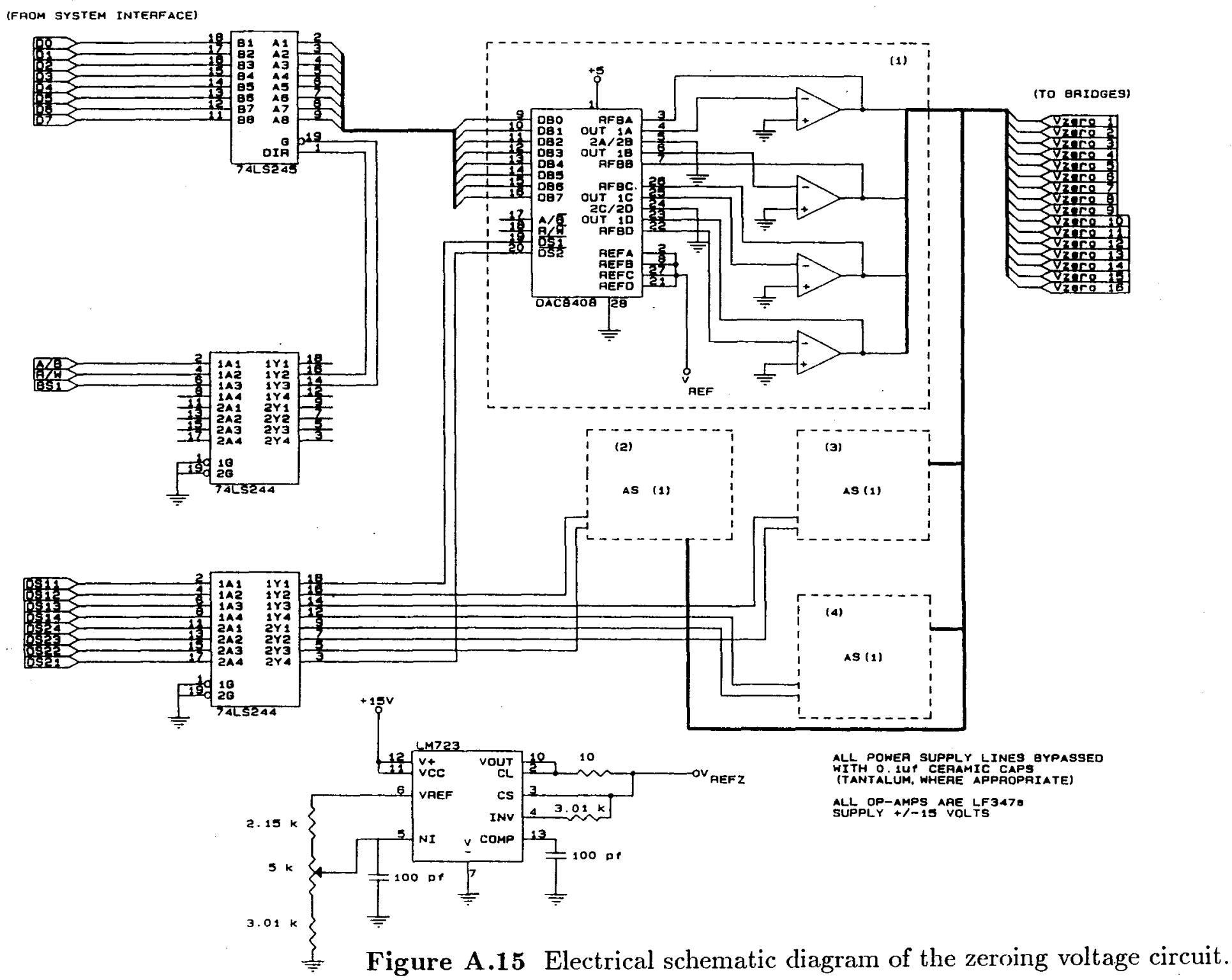




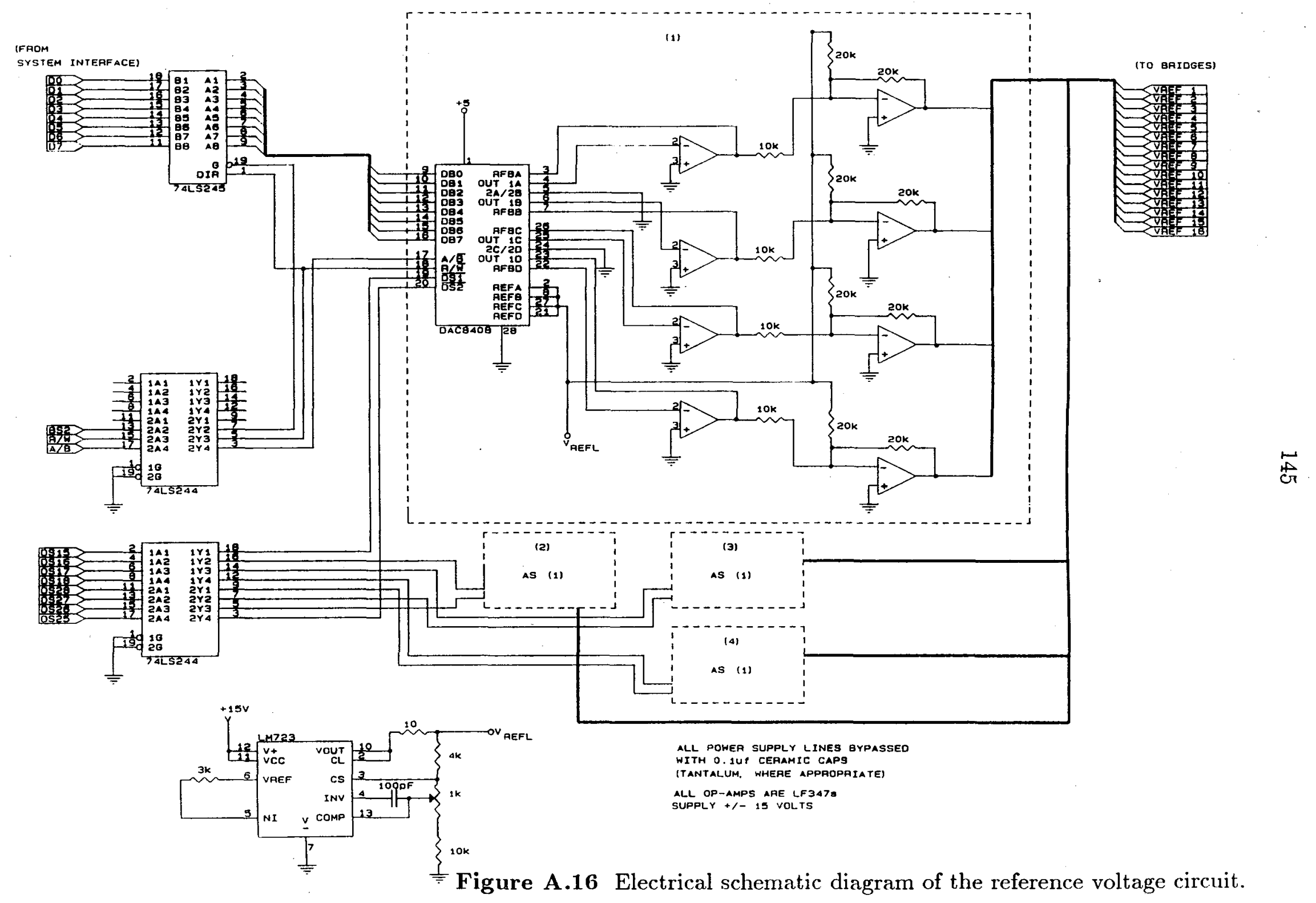



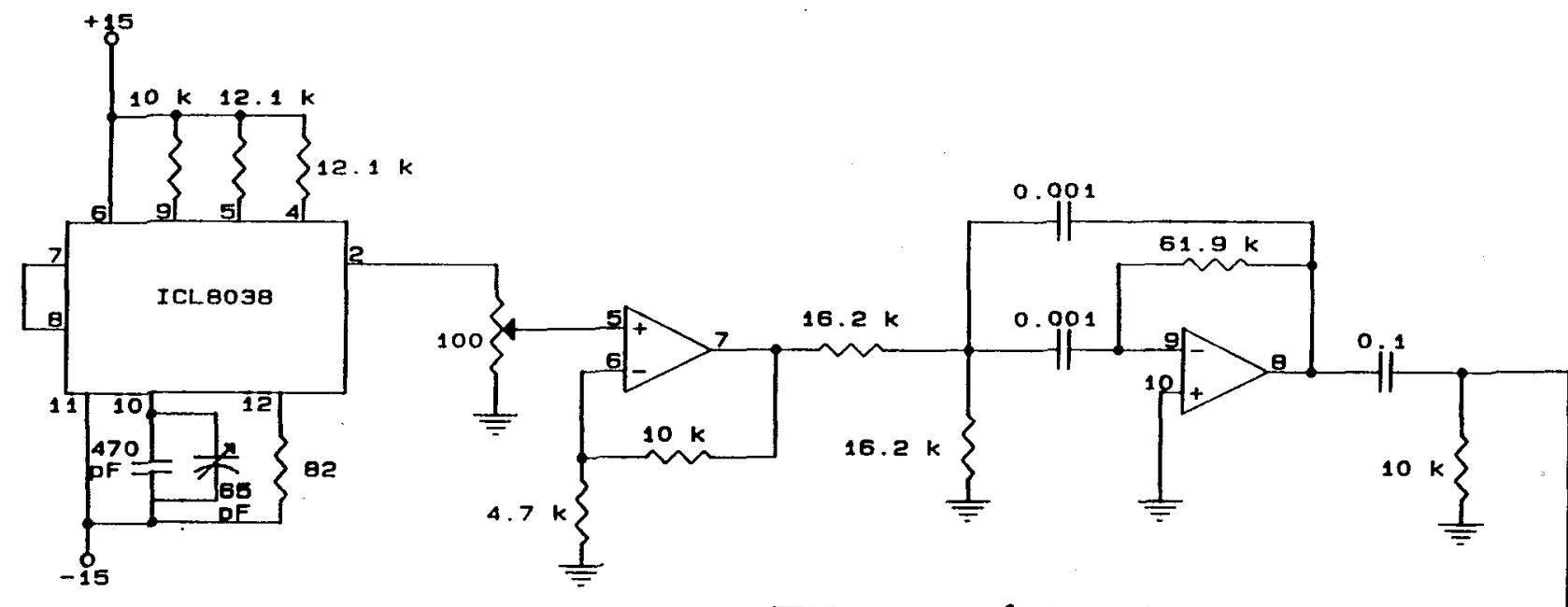
WITH O. 1Uf CERAMIC CAPS ALL OP-AMPS APE LF3478 suppiy $+/-15$ volts

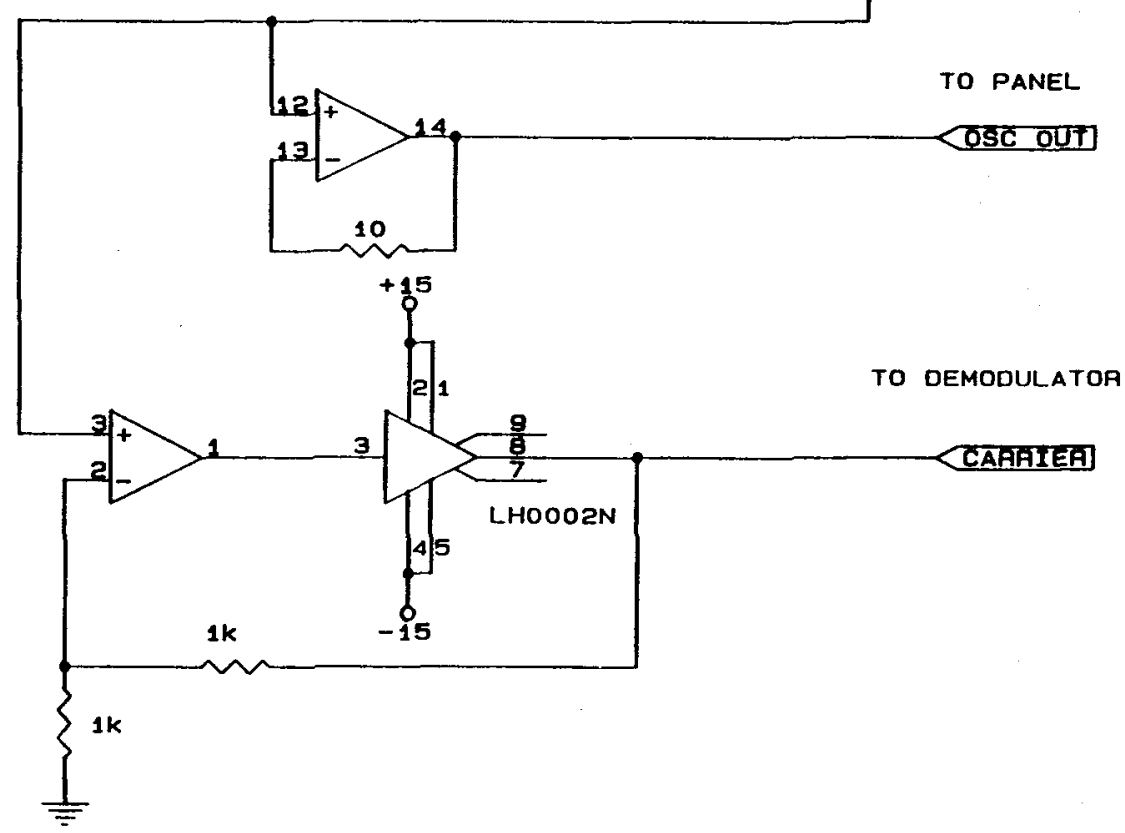

Figure A.17 Electrical schematic diagram of the carrier generator. 
To select signal output, with panel set to "computor," use these BASIC commands:

OUT $31 \mathrm{Bh}, 0 \mathrm{~h}$ for bridge null

OUT $31 \mathrm{Bh}, 1 \mathrm{~h}$ for probe signal

OUT $31 \mathrm{Bh}, 2 \mathrm{~h}$ for a.c. probe signal

OUT $31 \mathrm{Bh}, 3 \mathrm{~h}$ for d.c. probe signal

To set the zero DAC for a given bridge, use OUT $31 \mathrm{Eh}, \alpha$, then OUT $31 \mathrm{Fh}, n$, where $n$ is a number from 0 to 255 . For reference DAC, replace $\alpha$ with $\gamma$.

To input the zero DAC setting for a given bridge, use OUT $31 \mathrm{Eh}, \beta$, then $\operatorname{INP}(31 \mathrm{Fh})=n$, where $n$ is a number from 0 to 255 . For reference $\mathrm{DAC}$, replace $\beta$ with $\delta$.

\begin{tabular}{lllll} 
Bridge & $\alpha$ & $\beta$ & $\delta$ \\
\hline 0 & $4 \mathrm{~h}$ & $6 \mathrm{~h}$ & $\mathrm{C} 4 \mathrm{~h}$ & $\mathrm{C} 6 \mathrm{~h}$ \\
1 & $5 \mathrm{~h}$ & $7 \mathrm{~h}$ & $\mathrm{C} 5 \mathrm{~h}$ & $\mathrm{C} 7 \mathrm{~h}$ \\
2 & $\mathrm{Ah}$ & $\mathrm{C} 8 \mathrm{~h}$ & $\mathrm{CAh}$ \\
3 & Bh & C9h & CBh \\
4 & $16 \mathrm{~h}$ & D4h & D6h \\
5 & $14 \mathrm{~h}$ & $17 \mathrm{~h}$ & D5h & D7h \\
6 & $15 \mathrm{~h}$ & D8h & DAh \\
7 & $18 \mathrm{~h}$ & $1 \mathrm{Ah}$ & D9h & DBh \\
8 & $19 \mathrm{~h}$ & $26 \mathrm{~h}$ & E4h & E7h \\
9 & $24 \mathrm{~h}$ & $27 \mathrm{~h}$ & E5h & EAh \\
10 & $25 \mathrm{~h}$ & $2 \mathrm{Ah}$ & E8h & EBh \\
11 & $28 \mathrm{~h}$ & $2 \mathrm{Bh}$ & E9h & F6h \\
12 & $29 \mathrm{~h}$ & $36 \mathrm{~h}$ & F4h & F7h \\
13 & $34 \mathrm{~h}$ & $37 \mathrm{~h}$ & F5h & FAh \\
14 & $35 \mathrm{~h}$ & $3 \mathrm{Ah}$ & F8h & FBh \\
15 & $38 \mathrm{~h}$ & $3 \mathrm{Bh}$ & F9h &.
\end{tabular}

Figure A.18 Digital codes for the controller system. 


\section{Appendix B}

\section{Observations of Attached Cavities}

\section{Introduction}

This appendix presents some measurements of the dynamics and acoustics of attached cavities. Information regarding the dynamics of this kind of cavitation is important in a number of different applications. As discussed by Weitendorf (1989) it is critical to the understanding of ship propellor/hull interactions. It is also of importance to the understanding of the acoustic signal generated by ship propellors (Blake et al. (1977)) and the dynamic behavior of cavitating pumps (Brennen and Acosta (1976)). The dynamics of attached cavities has been difficult to study due to the absence of simple, non-intrusive volume measurement techniques. In the present study the fluid impedance measurement technique is used to provide a measurement of the cavity volume fluctuations on the axisymmetric bodies used to study attached cavitation. In other contexts, the electrodes could be used on either steady or moving surfaces as well as on surfaces that are geometrically complex.

\section{Experimental Methods}

Attached cavities were produced on an axisymmetric headform; the body used was the Schiebe headform described above. The body was constructed of lucite and was instrumented with three surface electrodes made of silver epoxy and located at positions $\mathrm{s} / \mathrm{D}=0.608,0.645$, and 0.691 . The surfaces of the body, including the electrode surface and electrode-lucite interface were highly polished. The interior of the headform contained water and an ITC-1042 hydrophone (Figure B.1). The acoustic impedance of lucite and water are nearly matched, thus reducing the attenuation due to reflection on the body surface. With this hydrophone geometry, external flow noise was reduced, and the dispersive effects of free stream bubbles were minimized. The headforms were supported by a two bladed sting with a nominally zero degree yaw angle. 
The experiments were conducted in the Caltech Low Turbulence Water Tunnel (Gates (1977)). In all the tests, the velocity of the tunnel was maintained constant and the pressure slowly reduced to approach the final operating point. The controlled air content of the water was 6-7 ppm for the results presented here.

Flash photographs of the attached cavities were taken for each operating point. The examples shown in Figure B.2 are for a tunnel velocity of $9 \mathrm{~m} / \mathrm{s}$ and cavitation numbers of $0.40,0.38,0.36$, and 0.34 . The cavity (or cavities) can be measured from these photographs in addition to the mean cavity length and thickness. Various cavity surface structures such as surface waves and cavity "fingering" may also be seen.

The internal hydrophone detected the noise made by the cavities. The signal was amplified and low pass filtered with a cut off of $100 \mathrm{kHz}$ and digitized at a sampling rate of $1 \mathrm{MHz}$. The high frequency content of the signal is limited by the response of the hydrophone, which is flat to approximately $80 \mathrm{kHz}$. Furthermore, the entire noise measurement is strongly affected by reverberation in the tunnel, especially in the frequency range up to $5 \mathrm{kHz}$ where the fundamental acoustic modes of the tunnel are located. By placing the hydrophone inside of the bluff body, the signal-to-noise ratio was significantly improved, and the affect of free stream bubbles on the acoustic signal was reduced.

The electrodes were used to measure both the mean volume and the volume fluctuations of the attached cavities. An alternating potential is applied to each electrode with the center electrode voltage being 180 degrees out of phase with the others. Changes in the center electrode current are detected and recorded. When a void is present over a portion of an electrode, two separate effects may change the signal. First, the percentage of the surface area of the electrode which will freely conduct electricity is reduced, and hence the current decreases. This is the primary signal detected by the electrode system. There may also be a secondary effect due to changes in conductivity of the cavity contents caused by the presence of a liquid 
and vapor mixture. The dynamic response of the electrode signal processor is on the order of $10 \mathrm{kHz}$, and the signal-to-electrical noise ratio was at least $45 \mathrm{~dB}$.

\section{Dynamics of Attached Cavities}

The formation of attached cavities was intermittent and occurred at seemingly random locations on the circumference of the body. Because all the experiments were conducted in a fairly narrow range of Reynolds number $\left(R e=4.4 \times 10^{5}-4.8 \times\right.$ $10^{5}$ ), the cavitation formation index for all experiments was about $\sigma=0.40$; the cavitation was located at about $s / D=0.45$. The attached cavity formation index is defined by the first appearance of attached cavitation anywhere on the headform. The cavitation disappearance index, which was always greater than the formation index because of the hysteresis effect, was about $\sigma=0.42$.

The photographs taken at each operating point were used as a reference for the acoustic and electrode data. At first formation the headform circumference was only about half covered with the attached cavities. Portions of the cavity were stable while others were intermittent. As the cavitation number was decreased, the cavities expanded to cover the entire circumference of the body. Further decrease in the cavitation number increased the length of the cavity, which is plotted against cavitation number in Figure B.3. The surface of the cavity shows a transition from a smooth laminar interface to a wavy and then a turbulent surface in a manner described and investigated by Brennen (1970). The point of transition on the cavity surface was about one half of the total cavity length in all cases. The cavity was composed of a series of longitudinal "finger" cavities which, at lower cavitation numbers, combined to cover the entire circumference.

Both the mean and fluctuating components of the electrode output in the current experiment contain interesting information. First, the mean level of the electrode signal is an indication of the percentage of the circumference that is covered by the cavity, and this is presented as a function of cavitation number in 
Figure B.4. As the cavitation number decreases, the percentage of the circumference covered by the cavity increases and hence the electrode signal voltage increases. It levels off as the cavity becomes fully developed. At high cavitation numbers, the large uncertainty represented by the large standard deviation is due to the temporal intermittency of the cavitation. Once the cavity is fully developed, the uncertainty decreases.

Secondly, the fluctuating component of the electrode signal was analysed. Initial spectra obtained without filtering indicated that there were no frequencies of significant magnitude above $500 \mathrm{~Hz}$. Therefore, the fluctuating signal was low pass filtered with a cutoff frequency of $1 k \mathrm{~Hz}$ and was digitally sampled at $2 \mathrm{kHz}$. This filtering eliminated the signals produced by the travelling bubbles that may form on portions of the electrode not covered by the cavity. Figure B.5 represents two typical spectra. They all have a similar shape with large amplitudes at low frequency and a uniform roll off to approximately $500 \mathrm{~Hz}$. Among other things this means that the interfacial stability waves described by Brennen (1970) do not contribute significantly, since using the observations of Brennen we estimate that those frequencies are in the range of 5 top $10 \mathrm{kHz}$.

At the higher cavitation numbers, the frequencies below $1 \mathrm{~Hz}$ are dominant. The temporal intermittency associated with the partially developed cavities produces this low frequency component. As the cavities become fully developed, these low frequencies become less pronounced. However, an intermediate frequency oscillation may be found in the spectra for the partially developed cavities. As seen in Figure B.5, the spectra for $\sigma=0.40$ has distinct frequency peaks (for example at $f=40,103$, and $133 \mathrm{~Hz}$ ). These peaks are not due to line noise, are repeatable, and disappear after the cavity becomes fully developed. Since they occur only when the cavity is partially developed, they may be due to pulsation of the finger cavities (see Figure B.6).

After the cavity is fully developed, the mean level of the signal fluctuations 
has decreased, but it is still significant. Since the electrodes were positioned under the laminar cavity region, where the cavity may be considered evacuated, these fluctuations are mainly due to two processes. Since the fully developed cavity consists of a sum of individual "finger" cavities, the surfaces of the fingers wet the electrode surface, and as these boundaries fluctuate, a signal is generated. Secondly, the cavity surface may intermittently collapse, wetting the surface and producing a signal. These spectra exhibit no dominant frequencies.

\section{Acoustics of Attached Cavities}

Acoustic spectra of the signal from the internal hydro- phone were generated for all operating conditions. The largest portion of the acoustic energy was found in the low frequency range from 0 to $5 k H z$ where the dominant reverberant modes of the tunnel are located. Hence, the spectra up to $5 k H z$ are of limited value. Figure B.7 presents the spectra for the cavitating conditions of $\sigma=0.40$ and $\sigma=0.32$ and for the non-cavitating background condition at $\sigma=1.0$, all at a tunnel velocity of $9.5 \mathrm{~m} / \mathrm{s}$. The background spectra has an approximately $20 \mathrm{~dB}$ falloff with resonant peaks at higher frequencies. These resonant peals can be related to specific acoustic path lengths in the water tunnel test section. For $\sigma=0.40$ the cavitation was partially developed, and for $\sigma=0.32$ the cavity was fully developed. Apart from the background peaks, the cavitating spectra magnitudes are generally at least 20 to $40 d B$ greater than the background. The cavitation spectra have a similar shape that is significantly different from the background shape, and the magnitudes of the high frequency content for the fully developed cavity are lower than for the partially developed case. This could be due to a muffling effect the cavity may have on the noise reaching the hydrophone. A significant portion of the high frequency noise may be due to the collapse of small bubbles formed in the pressure recovery region where the cavity collapses. In the fully developed case, these bubbles would be further away from the hydrophone and the noise would be somewhat shielded by the cavity. This trend is the reverse of that found by Blake et al. (1977) for 
attached cavities on hydrofoils, where lower cavitation numbers led to an increase in the high frequency magnitudes. The shape of the spectra measured by Blake remains virtually constant with cavitation number, and is similar to his reported background. However, the hydrophone used by Blake was externally mounted and hence the signal detected would be subject to different reverberant or transmission effects than those in the present measurement.

\section{Conclusions}

Photographic and acoustic/pressure measurement are the traditional methods used to study attached cavities. Volume fluctuations are difficult to study acoustically since most of the cavity oscillation frequencies are below $1 \mathrm{kHz}$ and are therefore strongly influenced by tunnel reverberation. The hydrophone was placed inside the headform, and the background and cavitating spectra were differentiated. The cavitation noise spectra exhibited a consistent shape, although the magnitudes at higher frequencies did exhibit some variation with flow conditions.

The electrode technique described here measures the area of surface cavitation as well as any dynamic component which could cause intermittent fluid/surface contact. The fluctuating component of the electrode signal revealed wideband cavity oscillations over a range of frequencies up to $500 \mathrm{~Hz}$. But, in addition and somewhat remarkably, they also showed a series of quite specific and repeatable frequencies present in the dynamic signal produced by intermittent cavities. These frequencies are about 40,100 , and $130 \mathrm{~Hz}$ and do not appear to coincide with any other structural or acoustic frequency of the system. They appear to be frequencies associated with pulsation of the finger cavities.

Although only one electrode geometry was used in this experiment, many other geometries are possible. For example, electrodes arrayed along the length of the cavity could yield information concerning the transition process and the collapse and shedding process in the pressure recovery region. 


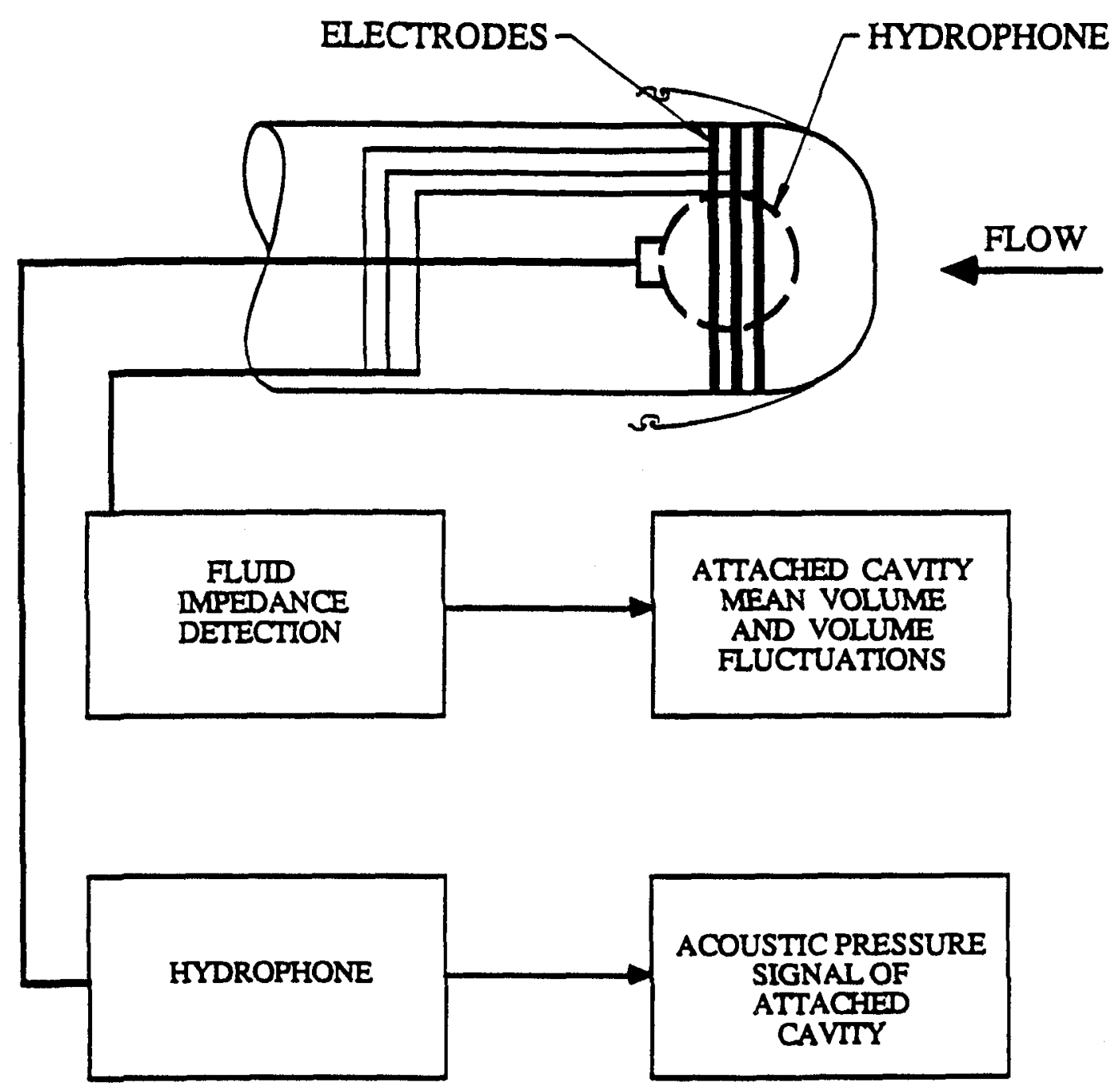

Figure B.1 Schematic diagram of the attached cavity experiment. 


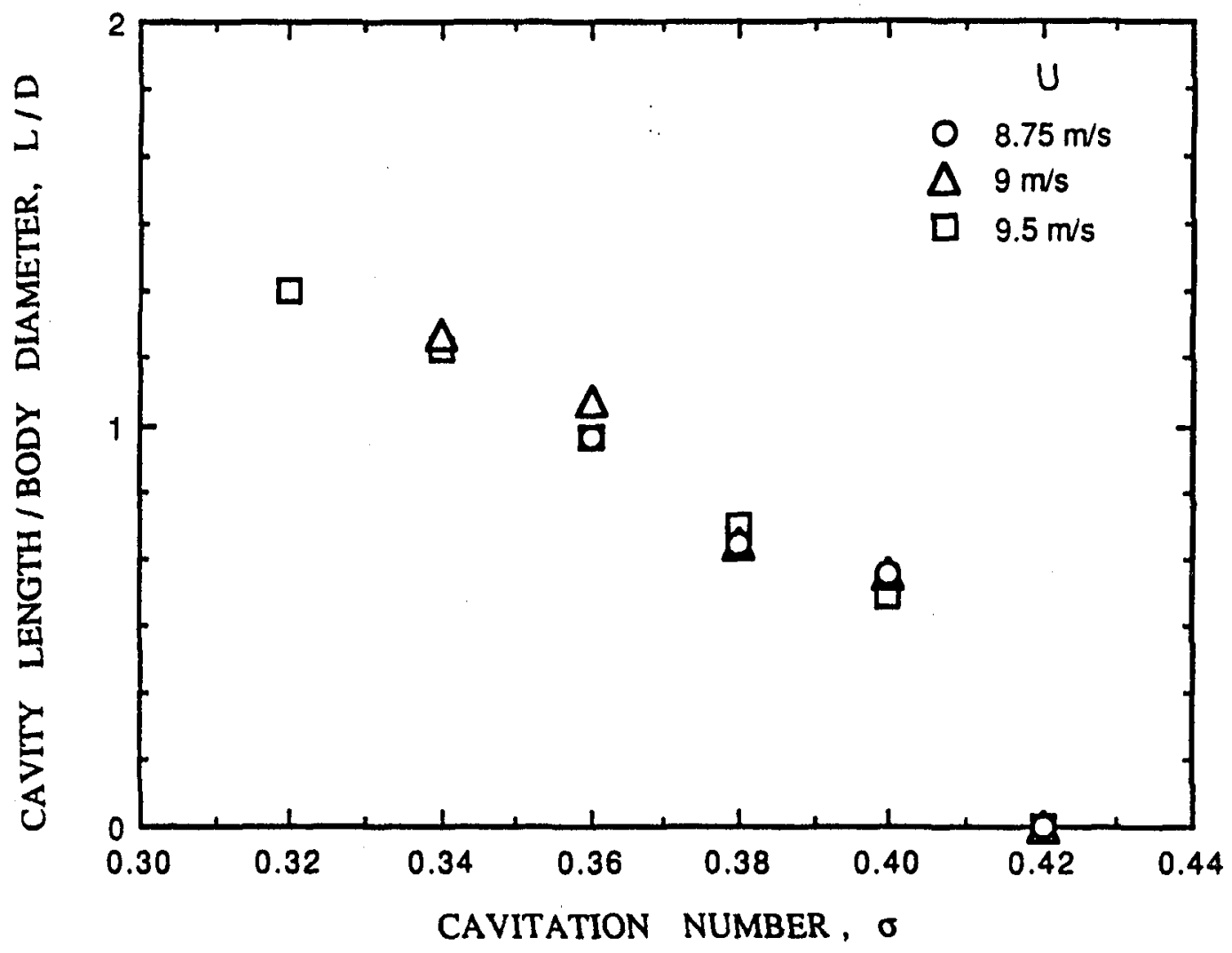

Figure B.3 Cavity length v. cavitation number for various tunnel velocities. 


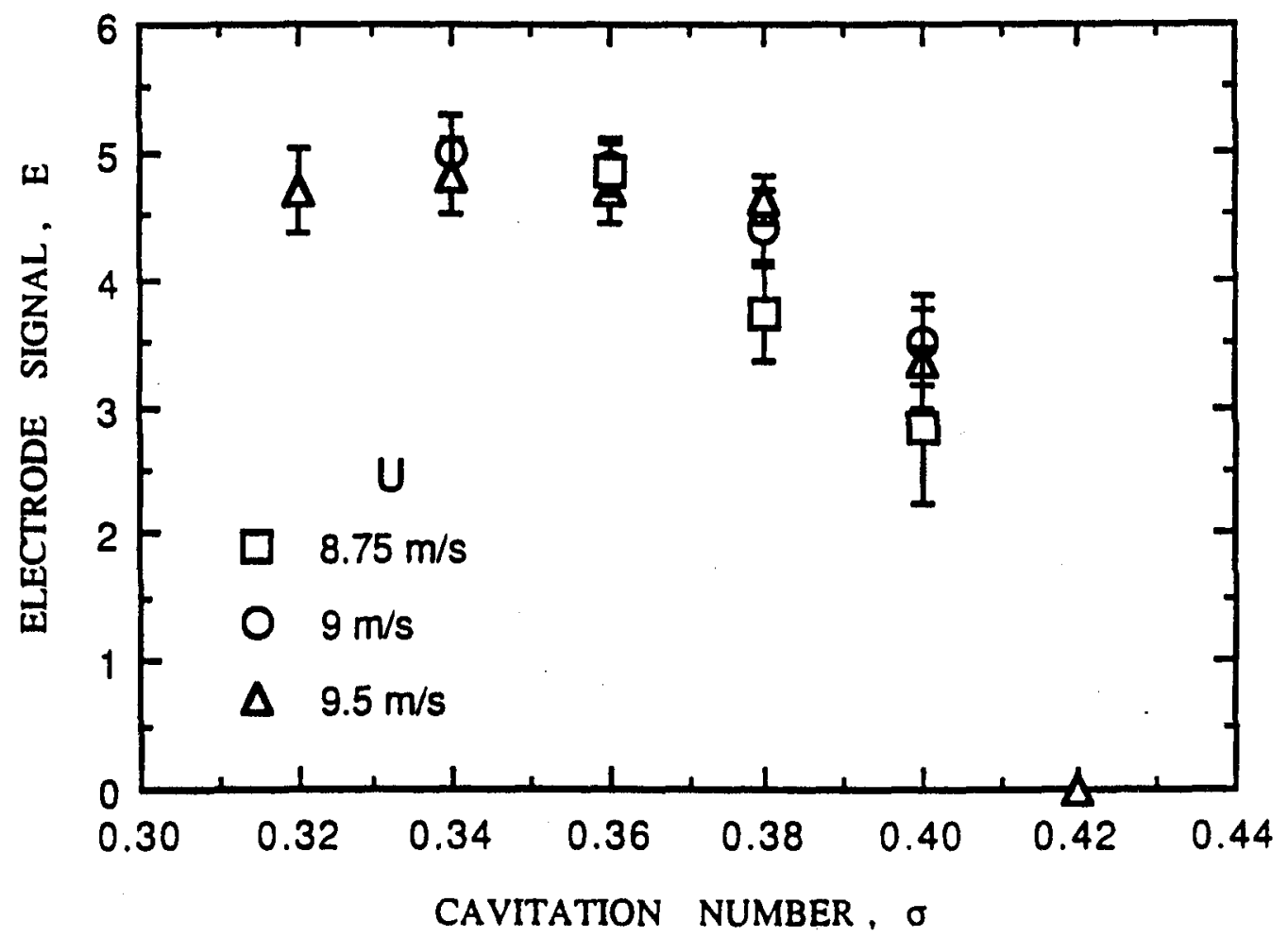

Figure B.4 Mean and standard deviation of the electrode signal v. cavitation number. Vertical scale is arbitrary. 

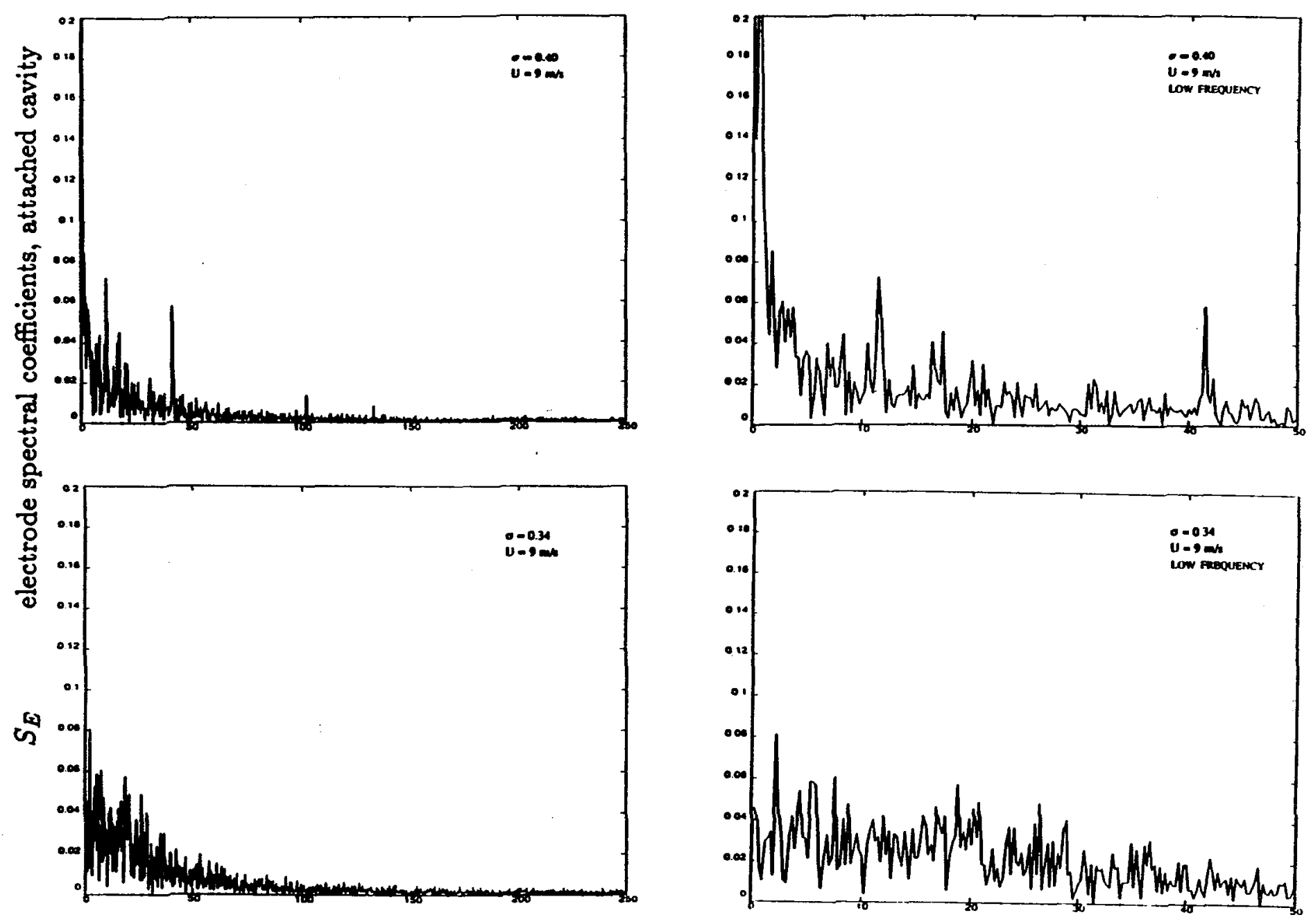

$\stackrel{\leftrightarrow}{\circ}$

frequency

Figure B.5 Electrode signal spectra for $\sigma=0.40$ and 0.34 at $U=9 \mathrm{~m} / \mathrm{s}$. Vertical scale is arbitrary. 


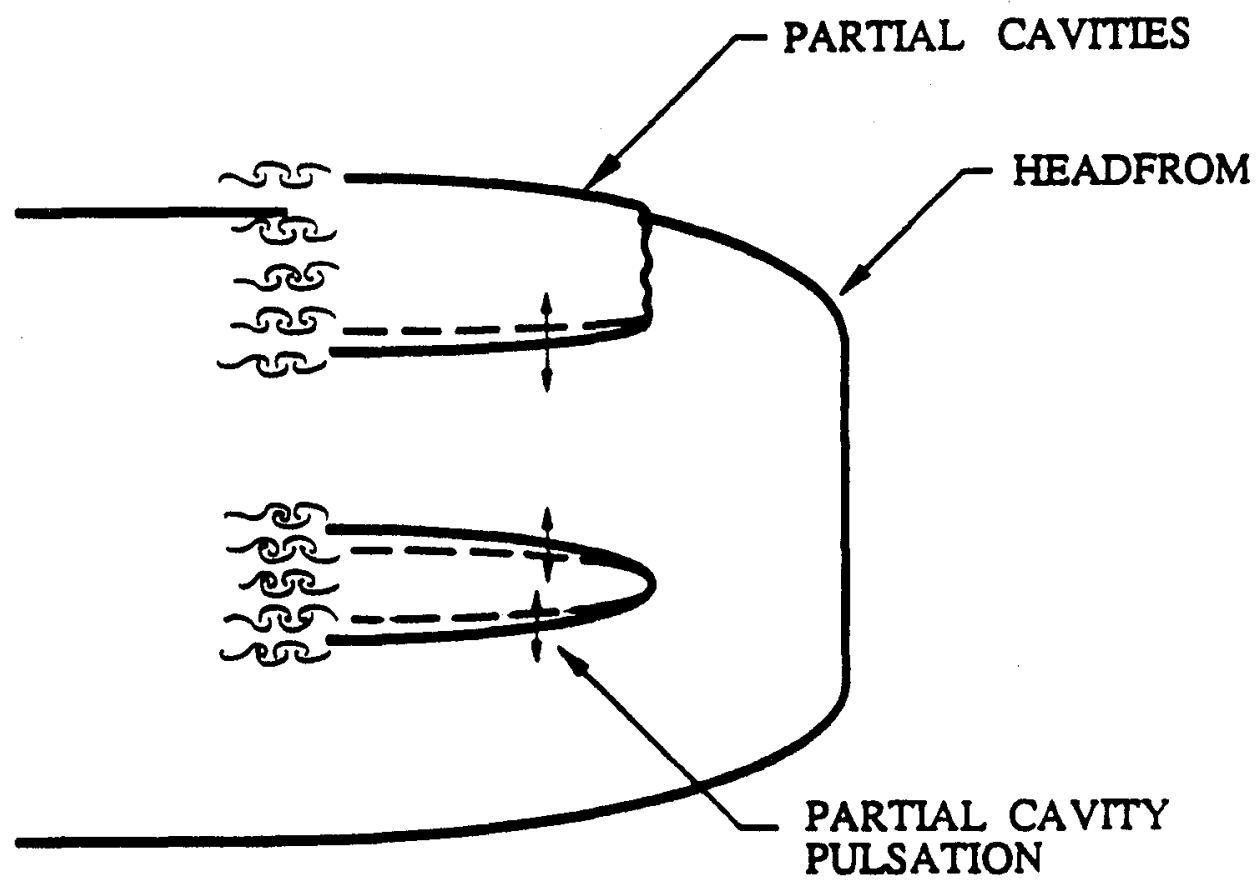

Figure B.6 One possible mode of cavity oscillation. 


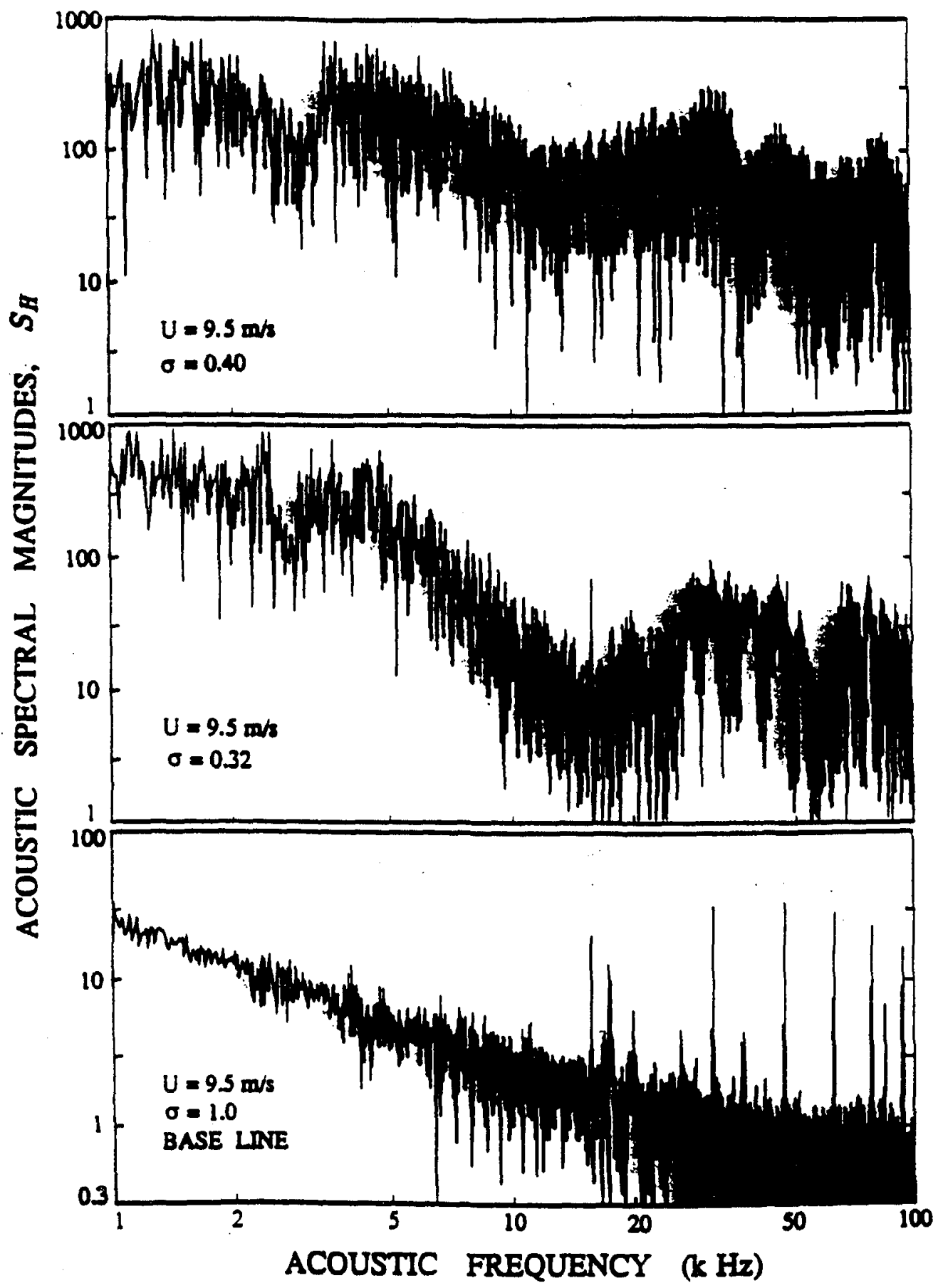

Figure B.7 Acoustic spectra for $\sigma=0.40$ and 0.32 at $U=9 \mathrm{~m} / \mathrm{s}$ and for $\sigma=1.0$ at $9.5 \mathrm{~m} / \mathrm{s}$. Vertical scale is arbitrary. 